\title{
LESSONS FROM THE GREEN LANES: EVALUATING PROTECTED BIKE LANES IN THE U.S.
}

\author{
FINAL REPORT: \\ APPENDIX A \\ SURVEY INSTRUMENTS
}

NITC-RR-583

\author{
by \\ Portland State University \\ Chris Monsere \\ Jennifer Dill \\ Nathan McNeil \\ Kelly Clifton \\ Nick Foster \\ Tara Goddard
}

for

National Institute for

Transportation and Communities (NITC)

P.O. Box 751

Portland, OR 97207

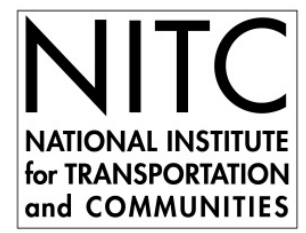

June 2014 


\section{ACKNOWLEDGEMENTS}

This research was funded by the National Institute for Transportation and Communities (NITC), a U.S. Department of Transportation university transportation center, People for Bikes (formerly Bikes Belong) and the Summit Foundation.

This research could not have been conducted without the significant participation of our city partners. These individuals provided data, design plans, conducted numerous reviews, and hosted our field visits: Mike Amsden (CDOT), David Smith (CDOT), Jim Sebastian (DDOT), Mike Goodno (DDOT), Roger Geller (PBOT), Rob Burchfield (PBOT), Ross Swanson (PBOT), Wendy Cawley (PBOT), Lindsay Walker (Lloyd District TMA), Seleta Reynolds (SFMTA), Miriam Sorell (SFMTA), Annick Beaudet (Austin), Nathan Wilkes (Austin), Aleksiina Chapman (Austin).

We acknowledge the efforts of the following Portland State University Students who assisted in survey mailing and video processing: Chase Ballew, Dan Stumpf, Dan Mercer, Lisa Okomoto, Allison Duncan, and Belinda Judelman. We also acknowledge the volunteers in each city that helped conduct the bicycle intercept survey.

Finally, thanks to anonymous peer reviewers who provided immensely helpful insights and corrections to this report (including suggesting the term "turning zone" which we adopted).

\section{DISCLAIMER}

The contents of this report reflect the views of the authors, who are solely responsible for the facts and the accuracy of the material and information presented herein. This document is disseminated under the sponsorship of the U.S. Department of Transportation University Transportation Centers Program in the interest of information exchange. The U.S. Government assumes no liability for the contents or use thereof. The contents do not necessarily reflect the official views of the U.S. Government. This report does not constitute a standard, specification, or regulation. 


\section{LIST OF SURVEYS}

Resident: Austin - Barton Springs

Resident: Austin - Bluebonnet

Resident: Chicago - Milwaukee Ave.

Resident: Chicago - Dearborn Ave.

Resident: Portland - NE Multnomah St.

Resident: San Francisco - Oak and Fell Streets

Resident: Washington DC - L Street

Bicyclist: Austin-Barton Springs

Bicyclist: Austin-Rio Grande

Bicyclist: Chicago-Milwaukee Ave.

Bicyclist: Chicago-Dearborn Ave.

Bicyclist: Portland - NE Multnomah St.

Bicyclist: San Francisco - Oak and Fell Streets

Bicyclist: Washington DC - L Street 



\section{RESIDENT: AUSTIN - BARTON SPRINGS}




\section{Neighborhood Street Study}

\section{About How You Get Around}

\begin{tabular}{|c|c|c|c|c|c|c|c|}
\hline \multirow{2}{*}{$\begin{array}{l}\text { 1. For each mode of transportation, } \\
\text { please indicate your level of use: }\end{array}$} & \multicolumn{3}{|c|}{$\begin{array}{c}\text { Commute Trips } \\
\text { (to/from work or school) }\end{array}$} & \multicolumn{4}{|c|}{$\begin{array}{c}\text { Other Trips } \\
\text { (e.g. to the store, park, etc.) }\end{array}$} \\
\hline & Most Trips & Some Trips & No Trips & M ost Trips & \multicolumn{2}{|c|}{ Some Trips } & No Trips \\
\hline Car/truck/motor vehicle (including carpool) & $\square_{1}$ & $\square_{2}$ & $\square_{3}$ & $\square_{1}$ & \multicolumn{2}{|c|}{$\square_{2}$} & $\square_{3}$ \\
\hline Walking & $\square_{1}$ & $\square_{2}$ & $\square_{3}$ & $\square_{1}$ & \multicolumn{2}{|c|}{$\square_{2}$} & $\square_{3}$ \\
\hline Bicycling & $\square_{1}$ & $\square_{2}$ & $\square_{3}$ & $\square_{1}$ & \multicolumn{2}{|c|}{$\square_{2}$} & $\square_{3}$ \\
\hline Public Transportation & $\square_{1}$ & $\square_{2}$ & $\square_{3}$ & $\square_{1}$ & \multicolumn{2}{|c|}{$\square_{2}$} & $\square_{3}$ \\
\hline Other (please specify): & $\square_{1}$ & $\square_{2}$ & $\square_{3}$ & $\square_{1}$ & \multicolumn{2}{|c|}{$\square_{2}$} & $\square_{3}$ \\
\hline \multicolumn{3}{|c|}{$\begin{array}{l}\text { 2. Compared to two years ago, are you taking more or } \\
\text { fewer trips by each mode of transportation? }\end{array}$} & More Trips & \multicolumn{2}{|c|}{ No Change } & \multicolumn{2}{|c|}{ Fewer Trips } \\
\hline \multicolumn{3}{|c|}{ Car/truck/motor vehicle (including carpool) } & $\square_{1}$ & \multicolumn{2}{|c|}{$\square_{2}$} & \multicolumn{2}{|c|}{$\square_{3}$} \\
\hline \multicolumn{3}{|c|}{ Walking } & $\square_{1}$ & \multicolumn{2}{|c|}{$\square_{2}$} & \multicolumn{2}{|r|}{$\square_{3}$} \\
\hline \multicolumn{3}{|r|}{ Bicycling } & $\square_{1}$ & \multicolumn{2}{|c|}{$\square_{2}$} & \multicolumn{2}{|r|}{$\square_{3}$} \\
\hline \multicolumn{3}{|c|}{ Public Transportation } & $\square_{1}$ & \multicolumn{2}{|c|}{$\square_{2}$} & \multicolumn{2}{|r|}{$\square_{3}$} \\
\hline \multicolumn{3}{|l|}{ Other (please specify): } & $\square_{1}$ & \multicolumn{2}{|l|}{$\square_{2}$} & & $\square_{3}$ \\
\hline
\end{tabular}
postage-paid envelope;

OR

Complete the online survey and raffle entry at:

http://tinyurl.com/BartonSpringsStreet

To take the online survey, you will need to enter this code: $\mathbf{5 0 0 0 8}$

Please complete the survey by $07 / 26 / 2013$
(1) Complete this paper survey and the enclosed raffle slip. Return in the

\section{About Your Neighborhood}

\section{Over the past two years, changes to my}

neighborhood as a place for ...

\begin{tabular}{|c|c|c|c|c|}
\hline $\begin{array}{c}\text { Very } \\
\text { Negative }\end{array}$ & Somewhat & No Impact/ & Somewhat & Very \\
No Changes & Positive & Positive \\
\hline$\square_{1}$ & $\square_{2}$ & $\square_{3}$ & $\square_{4}$ & $\square_{5}$ \\
$\square_{1}$ & $\square_{2}$ & $\square_{3}$ & $\square_{4}$ & $\square_{5}$ \\
$\square_{1}$ & $\square_{2}$ & $\square_{3}$ & $\square_{4}$ & $\square_{5}$ \\
$\square_{1}$ & $\square_{2}$ & $\square_{3}$ & $\square_{4}$ & $\square_{5}$ \\
\hline
\end{tabular}

taking public transportation have been ... .

walking have been . . .

driving have been ...

bicycling have been . .

4. Overall, my level of satisfaction with transportation in my neighborhood is...

\begin{tabular}{|c|c|c|c|}
\hline $\begin{array}{c}\text { Very } \\
\text { Dissatisfied }\end{array}$ & $\begin{array}{c}\text { Somewhat } \\
\text { Dissatisfied }\end{array}$ & $\begin{array}{c}\text { Somewhat } \\
\text { Satisfied }\end{array}$ & $\begin{array}{c}\text { Very } \\
\text { Satisfied }\end{array}$ \\
\hline$\square_{1}$ & $\square_{2}$ & $\square_{3}$ & $\square_{4}$ \\
\hline
\end{tabular}

About People You Encounter on the Street

5. Please indicate if you agree or disagree with the following statements:

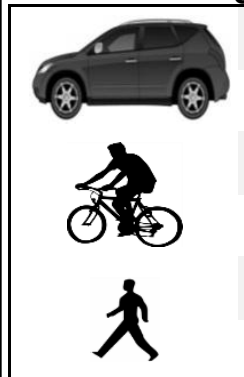

Most drivers follow the rules of the road.

M ost drivers are predictable.

Most bicyclists follow the rules of the road.

Most bicyclists are predictable.

Most pedestrians follow the rules of the road.

M ost pedestrians are predictable.

\begin{tabular}{|c|c|c|c|c|}
\hline $\begin{array}{c}\text { Strongly } \\
\text { Disagree }\end{array}$ & $\begin{array}{c}\text { Domewhat } \\
\text { Disagree }\end{array}$ & $\begin{array}{c}\text { Somewhat } \\
\text { Agree }\end{array}$ & $\begin{array}{c}\text { Strongly } \\
\text { Agree }\end{array}$ & $\begin{array}{c}\text { No } \\
\text { opinion }\end{array}$ \\
\hline$\square_{1}$ & $\square_{2}$ & $\square_{3}$ & $\square_{4}$ & $\square_{9}$ \\
$\square_{1}$ & $\square_{2}$ & $\square_{3}$ & $\square_{4}$ & $\square_{9}$ \\
$\square_{1}$ & $\square_{2}$ & $\square_{3}$ & $\square_{4}$ & $\square_{9}$ \\
$\square_{1}$ & $\square_{2}$ & $\square_{3}$ & $\square_{4}$ & $\square_{9}$ \\
$\square_{1}$ & $\square_{2}$ & $\square_{3}$ & $\square_{4}$ & $\square_{9}$ \\
$\square_{1}$ & $\square_{2}$ & $\square_{3}$ & $\square_{4}$ & $\square_{9}$ \\
\hline
\end{tabular}




\begin{tabular}{|c|c|c|c|c|c|}
\hline $\begin{array}{l}\text { 6. Please indicate whether you agree or disagree } \\
\text { with the following statements about bicycling: }\end{array}$ & $\begin{array}{l}\text { Strongly } \\
\text { Disagree }\end{array}$ & $\begin{array}{c}\text { Somewhat } \\
\text { Disagree }\end{array}$ & $\begin{array}{l}\text { Somewhat } \\
\text { Agree }\end{array}$ & $\begin{array}{l}\text { Strongly } \\
\text { Agree }\end{array}$ & $\begin{array}{c}\text { No } \\
\text { Opinion }\end{array}$ \\
\hline Bicycling in my city is a convenient way to get places. & $\square_{1}$ & $\square_{2}$ & $\square_{3}$ & $\square_{4}$ & $\square_{9}$ \\
\hline Bicycling in my neighborhood is safe. & $\square_{1}$ & $\square_{2}$ & $\square_{3}$ & $\square_{4}$ & $\square_{9}$ \\
\hline $\begin{array}{r}\text { I am familiar with the bicycle lanes and other bicycle } \\
\text { facilities in my neighborhood. }\end{array}$ & $\square_{1}$ & $\square_{2}$ & $\square_{3}$ & $\square_{4}$ & $\square_{9}$ \\
\hline I would like to bicycle more often for transportation. & $\square_{1}$ & $\square_{2}$ & $\square_{3}$ & $\square_{4}$ & $\square_{9}$ \\
\hline $\begin{array}{l}\text { I would be more likely to ride a bicycle if motor vehicles } \\
\text { and bicycles were physically separated by a barrier. }\end{array}$ & $\square_{1}$ & $\square_{2}$ & $\square_{3}$ & $\square_{4}$ & $\square_{9}$ \\
\hline $\begin{array}{l}\text { Facilities that encourage bicycling for transportation are } \\
\text { a good way to improve public health. }\end{array}$ & $\square_{1}$ & $\square_{2}$ & $\square_{3}$ & $\square_{4}$ & $\square_{9}$ \\
\hline
\end{tabular}

\section{Whether or not you currently ride a bicycle, please consider how comfortable you would be riding a bicycle in each place:}

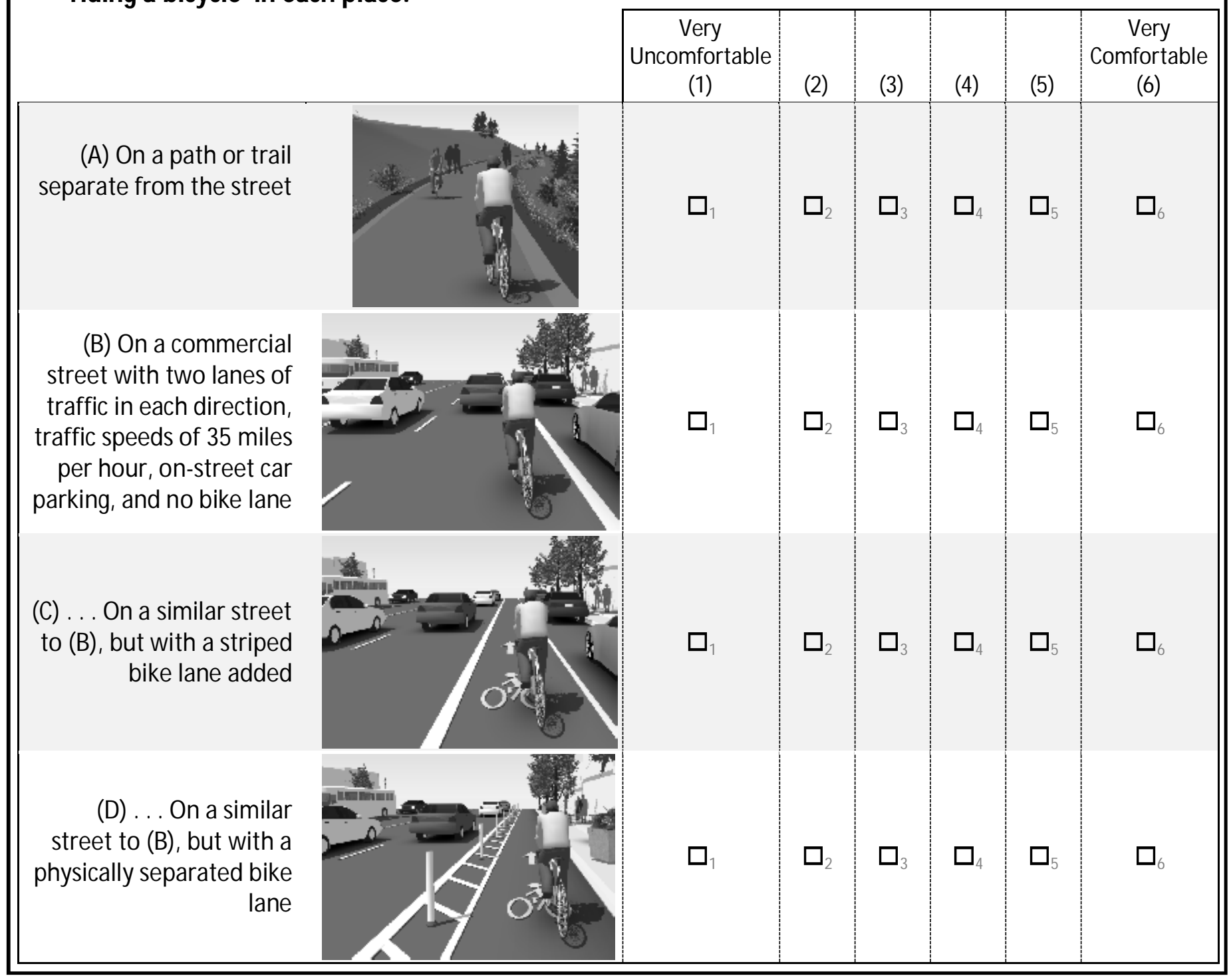




\section{Barton Springs Road Cycle Track}

A separated one-way eastbound bikeway, also known as a "cycle track," was recently built on Barton Springs Road from Lamar Boulevard to 1st Street. The cycle track includes a painted "buffer" area and plastic "flexposts" separating the cycle track from the standard traffic lane (see pictures below). The questions on the following pages are related to this cycle track. There will be questions related to the shared-use path on the north side of Barton Springs Road later on in this questionnaire.

\section{Barton Springs Road before and now:}
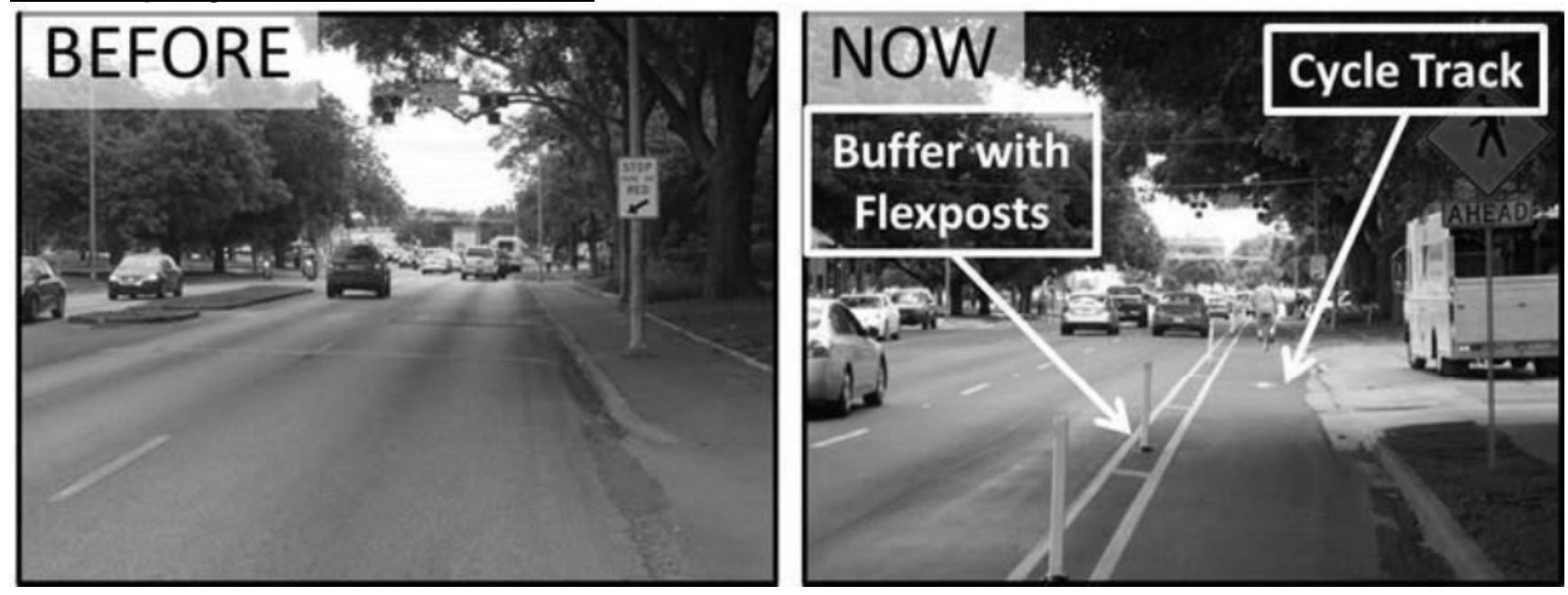

Extent of Barton Springs Road cycle track:

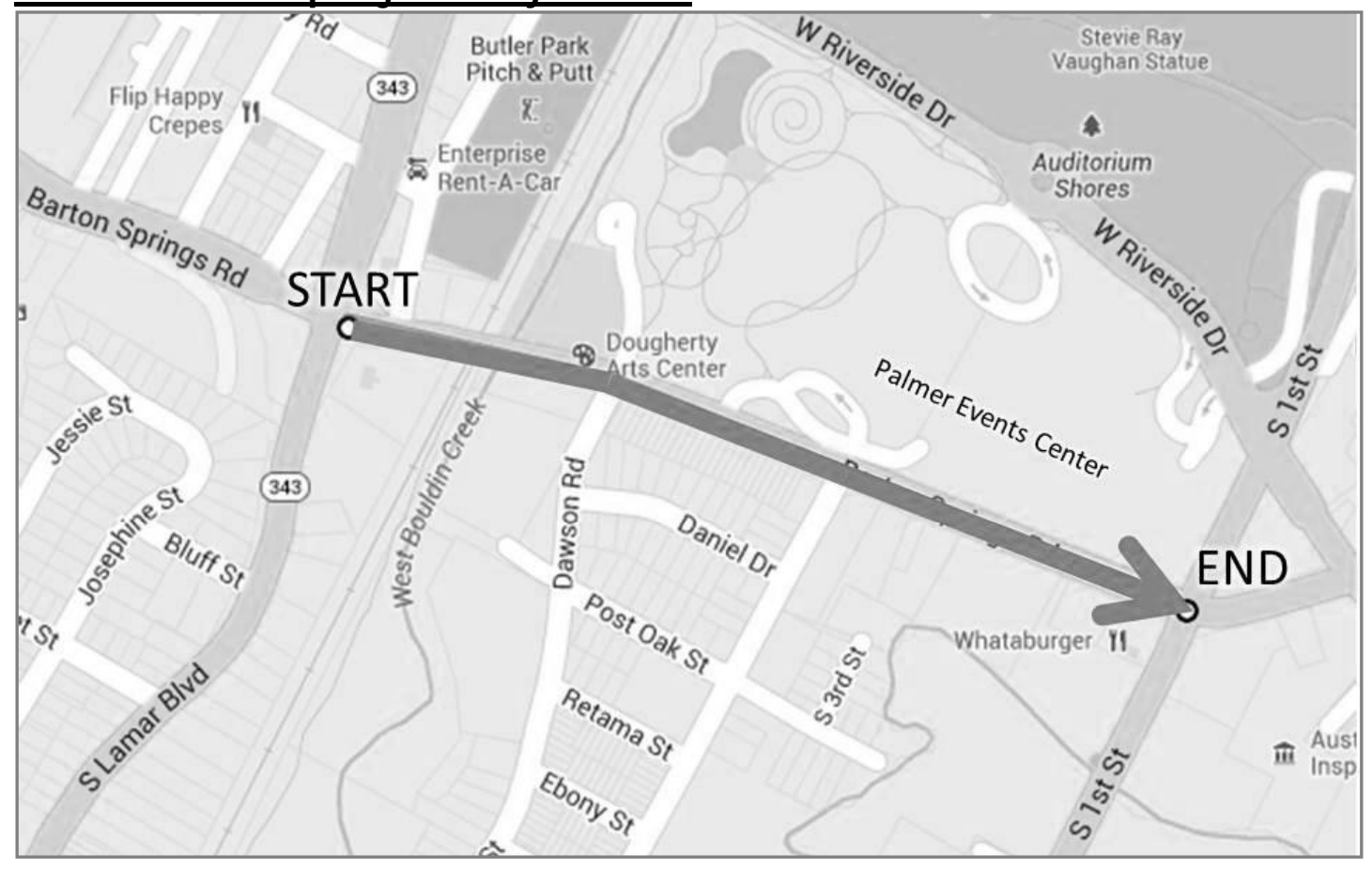


8. Because of the Barton Springs Road cycle track ...

\begin{tabular}{|c|c|c|c|c|c|c|}
\hline & $\begin{array}{c}\text { Decreased } \\
\text { a Lot }\end{array}$ & $\begin{array}{l}\text { Decreased } \\
\text { Somewhat }\end{array}$ & $\begin{array}{c}\text { Not } \\
\text { Changed }\end{array}$ & $\begin{array}{l}\text { Increased } \\
\text { Somewhat }\end{array}$ & $\begin{array}{c}\text { Increased } \\
\text { a Lot }\end{array}$ & $\begin{array}{c}\text { No } \\
\text { Opinion }\end{array}$ \\
\hline \multirow{5}{*}{$\begin{array}{r}\text { the aesthetic appeal of Barton Springs Road has... } \\
\text { the safety of DRIVING on Barton Springs } \\
\text { Road has ... } \\
\text { the safety of BICYCLING on Barton Springs } \\
\text { Road has. . } \\
\text { the safety of WALKING on Barton Springs } \\
\text { Road has ... } \\
\text { the number of people I see riding bikes } \\
\text { on Barton Springs Road has... }\end{array}$} & $\square_{1}$ & $\square_{2}$ & $\square_{3}$ & $\square_{4}$ & $\square_{5}$ & $\square_{9}$ \\
\hline & $\mathbf{\square}_{1}$ & $\square_{2}$ & $\square_{3}$ & $\square_{4}$ & $\square_{5}$ & $\square_{9}$ \\
\hline & $\square_{1}$ & $\square_{2}$ & $\square_{3}$ & $\square_{4}$ & $\square_{5}$ & $\square_{9}$ \\
\hline & $\square_{1}$ & $\square_{2}$ & $\square_{3}$ & $\square_{4}$ & $\square_{5}$ & $\square_{9}$ \\
\hline & $\mathbf{\square}_{1}$ & $\square_{2}$ & $\square_{3}$ & $\square_{4}$ & $\square_{5}$ & $\square_{9}$ \\
\hline how well the road works for all people has... & $\mathbf{\square}_{1}$ & $\square_{2}$ & $\square_{3}$ & $\square_{4}$ & $\square_{5}$ & $\square_{9}$ \\
\hline
\end{tabular}

\section{Please indicate whether you agree or disagree with the following statements about the} effectiveness of the Barton Springs Road cycle track:

The cycle track design makes it clear where cars can be and where the designated bicycle lanes are.

The buffer effectively separates bikes from cars. The buffer does a good job at protecting bikes from cars.

The cycle track improves the predictability of drivers and bicyclists.

The cycle track makes it clear where bicyclists and pedestrians should be.

The cycle track effectively separates bicyclists from pedestrians.

\begin{tabular}{|c|c|c|c|c|}
\hline $\begin{array}{c}\text { Strongly } \\
\text { Disagree }\end{array}$ & $\begin{array}{c}\text { Domewhat } \\
\text { Disagree }\end{array}$ & $\begin{array}{c}\text { Somewhat } \\
\text { Agree }\end{array}$ & $\begin{array}{c}\text { Strongly } \\
\text { Agree }\end{array}$ & $\begin{array}{c}\text { No } \\
\text { Opinion }\end{array}$ \\
$\square_{1}$ & $\square_{2}$ & $\square_{3}$ & $\square_{4}$ & $\square_{9}$ \\
$\square_{1}$ & $\square_{2}$ & $\square_{3}$ & $\square_{4}$ & $\square_{9}$ \\
$\square_{1}$ & $\square_{2}$ & $\square_{3}$ & $\square_{4}$ & $\square_{9}$ \\
$\square_{1}$ & $\square_{2}$ & $\square_{3}$ & $\square_{4}$ & $\square_{9}$ \\
$\square_{1}$ & $\square_{2}$ & $\square_{3}$ & $\square_{4}$ & $\square_{9}$ \\
$\square_{1}$ & $\square_{2}$ & $\square_{3}$ & $\square_{4}$ & $\square_{9}$ \\
\hline
\end{tabular}

10. Over time, my opinion of the Barton Springs Road cycle track has become:

\begin{tabular}{|c|c|c|c|c|}
\hline $\begin{array}{c}\text { A Lot More } \\
\text { Negative }\end{array}$ & $\begin{array}{c}\text { Somewhat } \\
\text { More Negative }\end{array}$ & No Change & $\begin{array}{c}\text { Somewhat } \\
\text { More Positive }\end{array}$ & $\begin{array}{c}\text { A Lot M ore } \\
\text { Positive }\end{array}$ \\
\hline$\square_{1}$ & $\square_{2}$ & $\square_{3}$ & $\square_{4}$ & $\square_{5}$ \\
\hline
\end{tabular}

11. Please indicate whether you agree or disagree with the following statements about cycle tracks.

\begin{tabular}{|r|c|c|c|c|c|}
\cline { 2 - 6 } \multicolumn{1}{c|}{} & $\begin{array}{c}\text { Strongly } \\
\text { Disagree }\end{array}$ & $\begin{array}{c}\text { Somewhat } \\
\text { Disagree }\end{array}$ & $\begin{array}{c}\text { Somewhat } \\
\text { Agree }\end{array}$ & $\begin{array}{c}\text { Strongly } \\
\text { Agree }\end{array}$ & $\begin{array}{c}\text { No } \\
\text { Opinion }\end{array}$ \\
\hline I would support building more cycle tracks at other \\
locations. & $\square_{1}$ & $\square_{2}$ & $\square_{3}$ & $\square_{4}$ & $\square_{9}$ \\
Overall, I support separating bikes from cars. & $\square_{1}$ & $\square_{2}$ & $\square_{3}$ & $\square_{4}$ & $\square_{9}$ \\
\hline
\end{tabular}




\section{About Driving on Barton Springs Road}

12. Have you driven a motor vehicle eastbound (toward 1st Street and Congress Avenue) on this section of Barton Springs Road since the cycle track was built?

$\square_{1}$ Yes (Proceed to Question 13)

$\square_{0}$ No (Skip to Question 18)

\section{Since the Barton Springs Road cycle track} was built ...

the number of bicyclists riding in the same lanes with cars on Barton Springs Road has...

how safe and predictable bicyclists are acting has ...

how safe and predictable drivers are acting has ...

the amount of time it takes me to drive on Barton Springs Road has... .

\begin{tabular}{|c|c|c|c|c|c|}
\hline $\begin{array}{c}\text { Decreased } \\
\text { a Lot }\end{array}$ & $\begin{array}{c}\text { Decreased } \\
\text { Somewhat }\end{array}$ & $\begin{array}{c}\text { Not } \\
\text { Changed }\end{array}$ & $\begin{array}{c}\text { Increased } \\
\text { Somewhat }\end{array}$ & $\begin{array}{c}\text { Increased } \\
\text { a Lot }\end{array}$ & $\begin{array}{c}\text { No } \\
\text { Opinion }\end{array}$ \\
\hline$\square_{1}$ & $\square_{2}$ & $\square_{3}$ & $\square_{4}$ & $\square_{5}$ & $\square_{9}$ \\
$\square_{1}$ & $\square_{2}$ & $\square_{3}$ & $\square_{4}$ & $\square_{5}$ & $\square_{9}$ \\
$\square_{1}$ & $\square_{2}$ & $\square_{3}$ & $\square_{4}$ & $\square_{5}$ & $\square_{9}$ \\
$\square_{1}$ & $\square_{2}$ & $\square_{3}$ & $\square_{4}$ & $\square_{5}$ & $\square_{9}$ \\
\hline
\end{tabular}

14. The impact of the Barton Springs Road cycle track on ...

traffic congestion has been ...

my ability to turn off of Barton Springs Road at signalized intersections has been . . .

my ability to turn off of Barton Springs Road into driveways has been ...

my ability to pull onto Barton Springs Road from driveways has been . .

\begin{tabular}{|c|c|c|c|c|}
\hline $\begin{array}{c}\text { Very } \\
\text { Negative }\end{array}$ & $\begin{array}{c}\text { Somewhat } \\
\text { Negative }\end{array}$ & $\begin{array}{c}\text { No Impact/ } \\
\text { Neutral }\end{array}$ & $\begin{array}{c}\text { Somewhat } \\
\text { Positive }\end{array}$ & $\begin{array}{c}\text { Very } \\
\text { Positive }\end{array}$ \\
\hline$\square_{1}$ & $\square_{2}$ & $\square_{3}$ & $\square_{4}$ & $\square_{5}$ \\
$\square_{1}$ & $\square_{2}$ & $\square_{3}$ & $\square_{4}$ & $\square_{5}$ \\
$\square_{1}$ & $\square_{2}$ & $\square_{3}$ & $\square_{4}$ & $\square_{5}$ \\
$\square_{1}$ & $\square_{2}$ & $\square_{3}$ & $\square_{4}$ & $\square_{5}$ \\
\hline
\end{tabular}

15. Please indicate if you agree or disagree with the following statements about turning across the cycle track.

When I want to make a left turn (across the cycle track), I am able to adequately see if there are any approaching bicyclists in the cycle track.

When I want to make a right turn (across the cycle track), I am able to adequately see if there are any approaching bicyclists in the cycle track.

\begin{tabular}{|c|c|c|c|c|}
\hline $\begin{array}{c}\text { Strongly } \\
\text { Disagree }\end{array}$ & $\begin{array}{c}\text { Somewhat } \\
\text { Disagree }\end{array}$ & $\begin{array}{c}\text { Somewhat } \\
\text { Agree }\end{array}$ & $\begin{array}{c}\text { Strongly } \\
\text { Agree }\end{array}$ & $\begin{array}{c}\text { No } \\
\text { Opinion }\end{array}$ \\
$\square_{1}$ & $\square_{2}$ & $\square_{3}$ & $\square_{4}$ & $\square_{9}$ \\
$\square_{1}$ & $\square_{2}$ & $\square_{3}$ & $\square_{4}$ & $\square_{9}$ \\
\hline
\end{tabular}

16. Do you avoid driving on Barton Springs Road because of the cycle track?
$\square_{1}$ Yes
$\square$ No 
About Bicycling on the Barton Springs Road Cycle Track

17. Have you bicycled eastbound (toward 1st Street and Congress Avenue) on this section of Barton Springs Road since the cycle track was built?

$\square_{1}$ Yes (Proceed to Question 18) $\square_{0}$ No (Skip to Question 23)

18. How comfortable do you feel when bicycling in the Barton Springs Road cycle track?

\begin{tabular}{|c|c|c|c|c|c|}
\hline $\begin{array}{c}\text { Very } \\
\text { Uncomfortable }\end{array}$ & & & & & $\begin{array}{c}\text { Very } \\
\text { Comfortable } \\
(1)\end{array}$ \\
\hdashline$\square_{1}$ & $\square_{2}$ & $\square_{3}$ & $\square_{4}$ & $\square_{5}$ & $\square_{6}$ \\
\hline
\end{tabular}

\section{Because of the Barton Springs Road} cycle track ...

\begin{tabular}{|l|l|c|l|l|l}
\hline Decreased Decreased & Not & Increased & Increased & No
\end{tabular}

the likelihood that I will choose to bicycle on Barton Springs as opposed to other streets has ... the time it takes me to bicycle on Barton Springs Road has...

the usefulness of Barton Springs Road for getting places I want to go has... how often I stop at shops and businesses on Barton Springs Road has ... drivers' awareness of bicyclists on Barton Springs Road has... drivers' speeds on Barton Springs Road have ... how comfortable I feel when bicycling on Barton Springs Road has...

how often I ride a bicycle overall has... the difficulty of turning left from Barton Springs Road while bicycling has... the difficulty of navigating around turning motor vehicles has ... the difficulty of navigating around pedestrians has...

how comfortable I feel bicycling with my kids has... how safe I feel bicycling with my kids has...

20. When bicycling EASTBOUND (toward 1st Street and Congress Avenue) on Barton Springs Road, how often do you ride on the following... . a Lot Somewhat Changed Somewhat a Lot Opinion

\begin{tabular}{l|l|l|l|l|l}
$\square_{1}$ & $\square_{2}$ & $\square_{3}$ & $\square_{4}$ & $\square_{5}$ & $\square_{9}$ \\
$\square_{1}$ & $\square_{2}$ & $\square_{3}$ & $\square_{4}$ & $\square_{5}$ & $\square_{9}$ \\
$\square_{1}$ & $\square_{2}$ & $\square_{3}$ & $\square_{4}$ & $\square_{5}$ & $\square_{9}$ \\
$\square_{1}$ & $\square_{2}$ & $\square_{3}$ & $\square_{4}$ & $\square_{5}$ & $\square_{9}$ \\
$\square_{1}$ & $\square_{2}$ & $\square_{3}$ & $\square_{4}$ & $\square_{5}$ & $\square_{9}$ \\
$\square_{1}$ & $\square_{2}$ & $\square_{3}$ & $\square_{4}$ & $\square_{5}$ & $\square_{9}$ \\
$\square_{1}$ & $\square_{2}$ & $\square_{3}$ & $\square_{4}$ & $\square_{5}$ & $\square_{9}$ \\
$\square_{1}$ & $\square_{2}$ & $\square_{3}$ & $\square_{4}$ & $\square_{5}$ & $\square_{9}$ \\
$\square_{1}$ & $\square_{2}$ & $\square_{3}$ & $\square_{4}$ & $\square_{5}$ & $\square_{9}$ \\
$\square_{1}$ & $\square_{2}$ & $\square_{3}$ & $\square_{4}$ & $\square_{5}$ & $\square_{9}$ \\
$\square_{1}$ & $\square_{2}$ & $\square_{3}$ & $\square_{4}$ & $\square_{5}$ & $\square_{9}$ \\
$\square_{1}$ & $\square_{2}$ & $\square_{3}$ & $\square_{4}$ & $\square_{5}$ & $\square_{9}$ \\
$\square_{1}$ & $\square_{2}$ & $\square_{3}$ & $\square_{4}$ & $\square_{5}$ & $\square_{9}$
\end{tabular}
Cycle track Standard traffic lanes Sidewalk Shared-use path on north side of Barton Springs Road

\begin{tabular}{|c|c|c|c|}
\hline Always & $\begin{array}{l}\text { Most of } \\
\text { the time }\end{array}$ & $\begin{array}{l}\text { Some of } \\
\text { the time }\end{array}$ & Never \\
\hline $\mathbf{\square}_{1}$ & $\square_{2}$ & $\square_{3}$ & $\square_{4}$ \\
\hline$\square_{1}$ & $\square_{2}$ & $\square_{3}$ & $\square_{4}$ \\
\hline $\mathbf{\square}_{1}$ & $\square_{2}$ & $\square_{3}$ & $\square_{4}$ \\
\hline $\mathbf{\square}_{1}$ & $\square_{2}$ & $\square_{3}$ & $\square_{4}$ \\
\hline
\end{tabular}

21. In the past $\mathbf{3 0}$ days, how many days have you bicycled for transportation (i.e. not just for recreation/ exercise)? 
22. Did you fill out a separate online bicyclist survey about the Barton Springs Road cycle track from us recently?
$\square_{1}$ Yes
$\square_{0}$ No

\section{About Walking on Barton Springs Road}

23. Have you walked on the south side of this section of Barton Springs Road since the cycle track was built?
$\square_{1}$ Yes (Proceed to Question 24)
$\square_{0}$ No (Skip to Question 26)

24. Because of the Barton Springs Road cycle track . . .

drivers' speeds on Barton Springs Road have generally ...

the number of bicyclists riding on the sidewalk has ...

my satisfaction with the walking environment on Barton Springs Road has ...

my sense of safety when crossing Barton Springs

Road has...

\begin{tabular}{|c|c|c|c|c|c|}
\hline $\begin{array}{c}\text { Decreased } \\
\text { a Lot }\end{array}$ & $\begin{array}{c}\text { Decreased } \\
\text { Somewhat }\end{array}$ & $\begin{array}{c}\text { Not } \\
\text { Changed }\end{array}$ & $\begin{array}{c}\text { Increased } \\
\text { Somewhat }\end{array}$ & $\begin{array}{c}\text { Increased } \\
\text { a Lot }\end{array}$ & $\begin{array}{c}\text { No } \\
\text { Opinion }\end{array}$ \\
\hline$\square_{1}$ & $\square_{2}$ & $\square_{3}$ & $\square_{4}$ & $\square_{5}$ & $\square_{9}$ \\
$\square_{1}$ & $\square_{2}$ & $\square_{3}$ & $\square_{4}$ & $\square_{5}$ & $\square_{9}$ \\
$\square_{1}$ & $\square_{2}$ & $\square_{3}$ & $\square_{4}$ & $\square_{5}$ & $\square_{9}$ \\
$\square_{1}$ & $\square_{2}$ & $\square_{3}$ & $\square_{4}$ & $\square_{5}$ & $\square_{9}$ \\
\hline
\end{tabular}

25. Do you ever walk or jog in the cycle track, rather than on the sidewalk?

$\square$ No

$\square_{1}$ Yes (Please explain why you choose the cycle track over the sidewalk in the space to the right) $\rightarrow$

\section{Overall Impressions of the Barton Springs Road Cycle Track}

26. What is the best thing about the cycle track?

27. What is the worst thing about the cycle track? 


\section{Barton Springs Road Shared-use Path}

Two new bike facilities were recently built on Barton Springs Road from Lamar Boulevard to 1st Street. We've already asked you about the cycle track on the south side of the road. The other of these facilities is a new one-way westbound shared-use path with a grass strip "buffer" area separating the path from the standard traffic lane and a striped lane separating people bicycling and walking (see pictures below). This path was created by widening the sidewalk on the north side of the road. The questions on the following pages are related to this shared-use path.

\section{Barton Springs Road north-side before and now:}
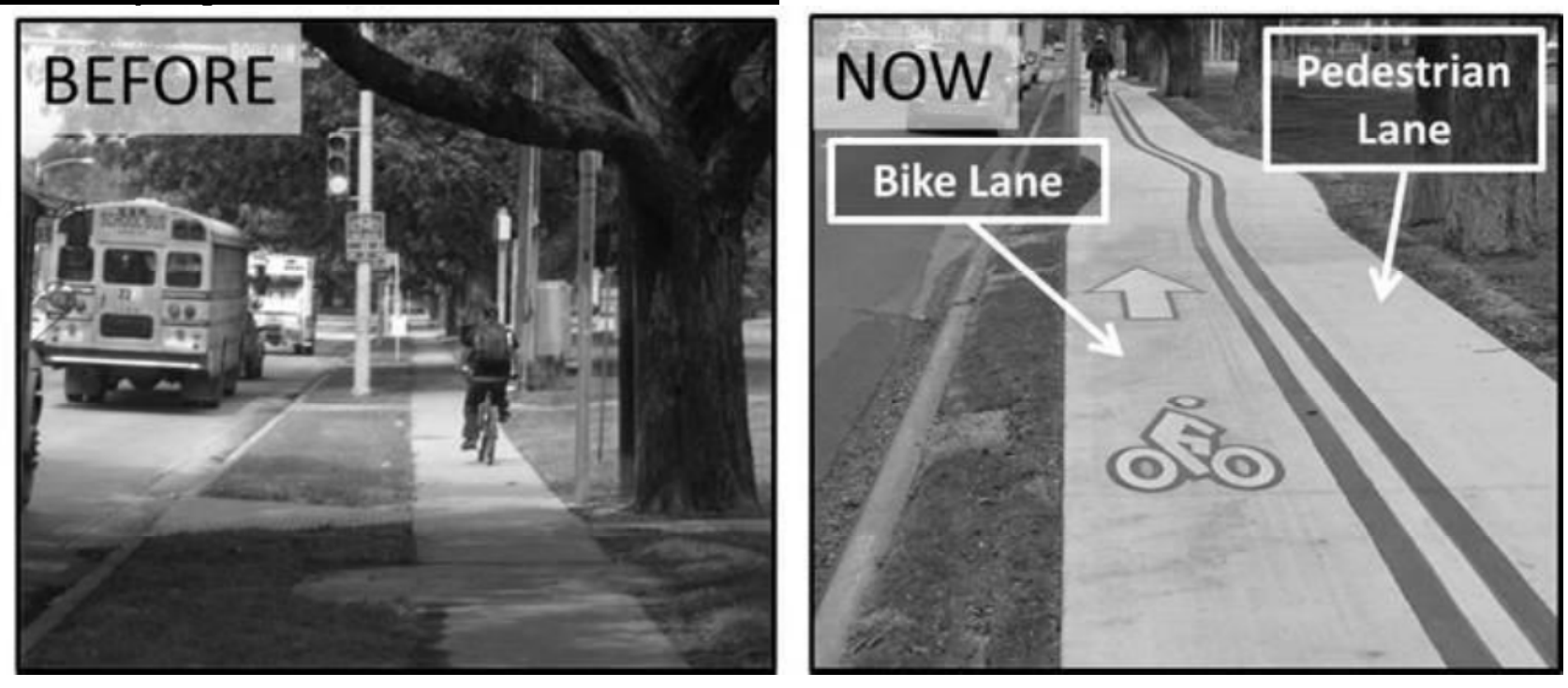

Extent of Barton Springs Road shared-use path:

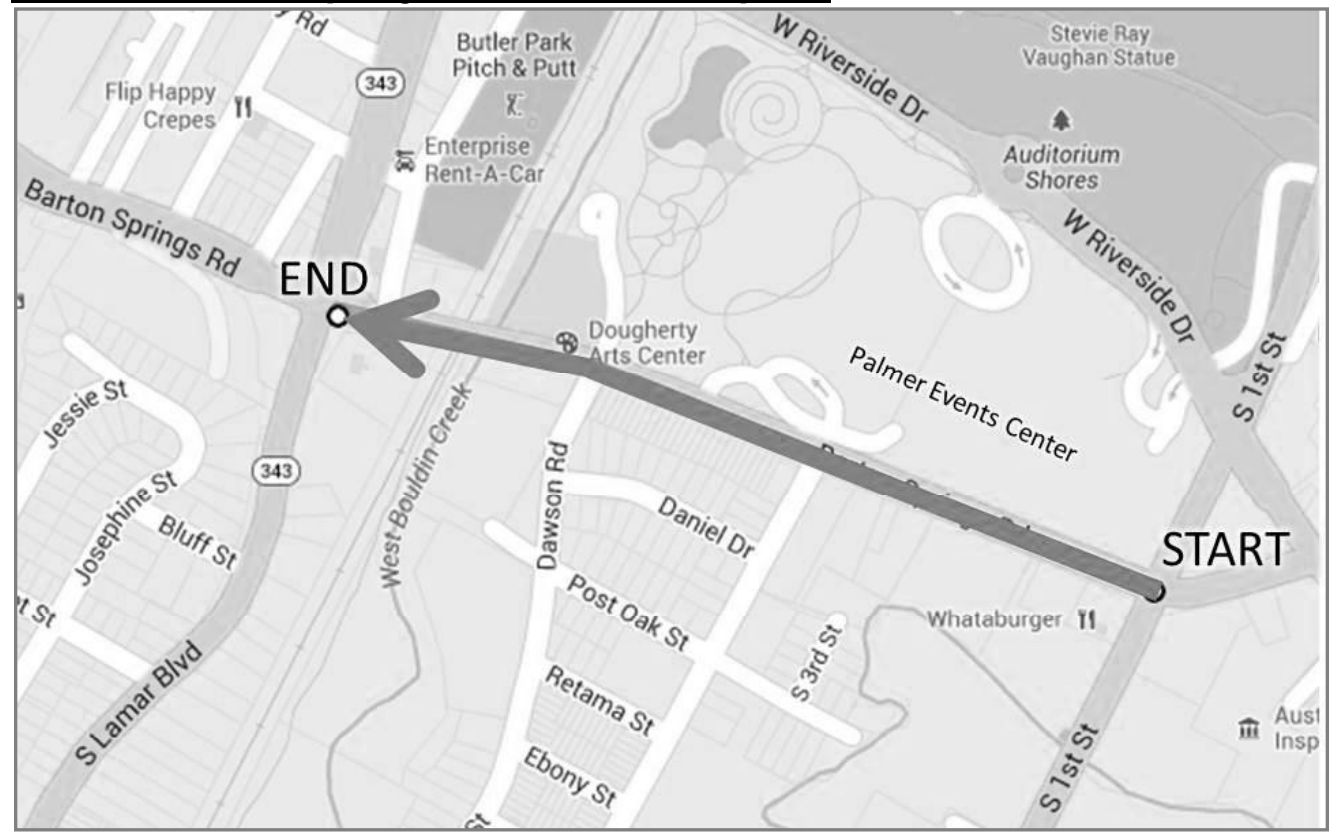


About Bicycling on the Barton Springs Road Shared-use Path

28. Have you bicycled on this shared-use path in the past three months?

$\square_{1}$ Yes (Proceed to Question 29) $\square_{0}$ No (Skip to Question 34)

\begin{tabular}{l|l|c|c|c|c|c|c|}
\hline $\begin{array}{l}\text { 29. How comfortable do you feel bicycling in } \\
\text { the Barton Springs Road shared-use path? }\end{array}$ & $\begin{array}{c}\text { Very } \\
\text { Uncomfortable } \\
\text { (1) }\end{array}$ & (2) & (3) & (4) & (5) & $\begin{array}{c}\text { Cery } \\
\text { Comfortable } \\
\text { (6) }\end{array}$ \\
\hline & $\square_{1}$ & $\square_{2}$ & $\square_{3}$ & $\square_{4}$ & $\square_{5}$ & $\square_{6}$ \\
\hline
\end{tabular}

30. Because of the Barton Springs Road shared-use path ...

I feel the safety of bicycling on Barton Springs Road has...

the time it takes me to bicycle on Barton Springs

Road has...

the usefulness of Barton Springs Road for getting places I want to go has...

how often I stop at shops and businesses on Barton Springs Road has... drivers' awareness of bicyclists on Barton Springs Road has...

how comfortable I feel when bicycling on Barton Springs Road has...

how often I ride a bicycle overall has... the difficulty of turning left from Barton Springs Road while bicycling has... the difficulty of navigating around turning motor vehicles has... the difficulty of navigating around pedestrians has...

how comfortable I feel bicycling with my kids has... how safe I feel bicycling with my kids has...

\begin{tabular}{|l|l|c|c|c|c|}
\hline Decreased & Decreased & Not & Increased & Increased & No
\end{tabular} a Lot Somewhat Changed Somewhat a Lot Opinion

\begin{tabular}{l|l|l|l|l|l|}
$\square_{1}$ & $\square_{2}$ & $\square_{3}$ & $\square_{4}$ & $\square_{5}$ & $\square_{9}$ \\
$\square_{1}$ & $\square_{2}$ & $\square_{3}$ & $\square_{4}$ & $\square_{5}$ & $\square_{9}$ \\
$\square_{1}$ & $\square_{2}$ & $\square_{3}$ & $\square_{4}$ & $\square_{5}$ & $\square_{9}$ \\
$\square_{1}$ & $\square_{2}$ & $\square_{3}$ & $\square_{4}$ & $\square_{5}$ & $\square_{9}$ \\
$\square_{1}$ & $\square_{2}$ & $\square_{3}$ & $\square_{4}$ & $\square_{5}$ & $\square_{9}$ \\
$\square_{1}$ & $\square_{2}$ & $\square_{3}$ & $\square_{4}$ & $\square_{5}$ & $\square_{9}$ \\
$\square_{1}$ & $\square_{2}$ & $\square_{3}$ & $\square_{4}$ & $\square_{5}$ & $\square_{9}$ \\
$\square_{1}$ & $\square_{2}$ & $\square_{3}$ & $\square_{4}$ & $\square_{5}$ & $\square_{9}$ \\
$\square_{1}$ & $\square_{2}$ & $\square_{3}$ & $\square_{4}$ & $\square_{5}$ & $\square_{9}$ \\
$\square_{1}$ & $\square_{2}$ & $\square_{3}$ & $\square_{4}$ & $\square_{5}$ & $\square_{9}$ \\
$\square_{1}$ & $\square_{2}$ & $\square_{3}$ & $\square_{4}$ & $\square_{5}$ & $\square_{9}$ \\
$\square_{1}$ & $\square_{2}$ & $\square_{3}$ & $\square_{4}$ & $\square_{5}$ & $\square_{9}$ \\
\hline & & &
\end{tabular}

\section{When bicycling WESTBOUND (toward Lamar} Boulevard and Barton Springs Pool) on Barton Springs Road, how often do you ride on the following ... .

\begin{tabular}{|c|c|c|c|c|}
\cline { 3 - 5 } \multicolumn{1}{c|}{} & & $\begin{array}{c}\text { Most of } \\
\text { the time }\end{array}$ & $\begin{array}{c}\text { Some of } \\
\text { the time }\end{array}$ & Never \\
\hline Ase path & $\square_{1}$ & $\square_{2}$ & $\square_{3}$ & $\square_{4}$ \\
Always & $\square_{1}$ & $\square_{2}$ & $\square_{3}$ & $\square_{4}$ \\
Road & $\square_{1}$ & $\square_{2}$ & $\square_{3}$ & $\square_{4}$ \\
\hline rad & $\square_{1}$ & $\square_{2}$ & $\square_{3}$ & $\square_{4}$ \\
\hline
\end{tabular}


About Bicycling on the Barton Springs Road Shared-use Path, cont.

32. In the past 30 days, how many days have you bicycled for transportation (i.e. not just for recreation/ exercise)?

33. Did you fill out a separate online bicyclist survey about the Barton Springs Road cycle track from us recently?

$\square_{1}$ Yes $\quad \square_{0}$ No

\section{About Walking on the Barton Springs Road Shared-use Path}

34. Have you walked on this shared-use path in the last three months?

$\square_{1}$ Yes (Proceed to Question 35) $\quad \square_{0}$ No (Skip to Question 36)

35. Because of the Barton Springs Road shared-use path ...

my satisfaction with the walking environment on Barton Springs Road has . . my sense of safety when crossing Barton Springs Road has... how often I have conflicts with bicyclists has...

\begin{tabular}{|c|c|c|c|c|c|}
\hline $\begin{array}{c}\text { Decreased } \\
\text { a Lot }\end{array}$ & $\begin{array}{c}\text { Decreased } \\
\text { Somewhat }\end{array}$ & $\begin{array}{c}\text { Not } \\
\text { Changed }\end{array}$ & $\begin{array}{l}\text { Increased } \\
\text { Somewhat }\end{array}$ & $\begin{array}{c}\text { Increased } \\
\text { a Lot }\end{array}$ & $\begin{array}{c}\text { No } \\
\text { Opinion }\end{array}$ \\
\hline$\square_{1}$ & $\square_{2}$ & $\square_{3}$ & $\square_{4}$ & $\square_{5}$ & $\square_{9}$ \\
$\square_{1}$ & $\square_{2}$ & $\square_{3}$ & $\square_{4}$ & $\square_{5}$ & $\square_{9}$ \\
$\square_{1}$ & $\square_{2}$ & $\square_{3}$ & $\square_{4}$ & $\square_{5}$ & $\square_{9}$ \\
\hline
\end{tabular}

\section{Overall Impressions of the Barton Springs Road Shared-use Path}

36. What is the best thing about the shared-use path?
37. What is the worst thing about the shared-use path? 


\section{About Visiting Businesses on Barton Springs Road}

38. How many days per month do you spend money at businesses on or near Barton Springs Road?

39. How did you travel to get there?

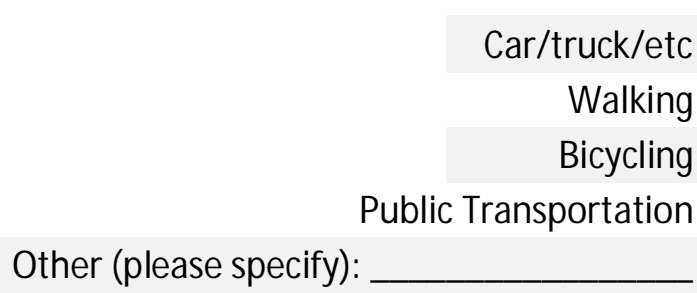

\begin{tabular}{|c|c|c|}
\hline $\begin{array}{c}\text { Most of the } \\
\text { time }\end{array}$ & $\begin{array}{c}\text { Some of the } \\
\text { time }\end{array}$ & Never \\
\hline$\square_{1}$ & $\square_{2}$ & $\square_{3}$ \\
$\square_{1}$ & $\square_{2}$ & $\square_{3}$ \\
$\square_{1}$ & $\square_{2}$ & $\square_{3}$ \\
$\square_{1}$ & $\square_{2}$ & $\square_{3}$ \\
$\square_{1}$ & $\square_{2}$ & $\square_{3}$ \\
\hline
\end{tabular}

40. What types of businesses in the area do you patronize? (check all that apply)

$\square_{1}$ Grocery Store

$\square$ Restaurants

$\square_{5}$ Banks

$\square_{2}$ Retail Shops

$\square_{4}$ Bars

$\square_{6}$ Coffee Shop

Other ->

41. When you visit this area, how many businesses do you typically go to each time? (select the best response)

$\square_{1} 1$

$\square_{2} 2$

$\square_{3} 3$ or more

42. Since the cycle track was built, are you more or less likely to visit a business on Barton Springs Road?
$\square_{1}$ A lot less likely
$\square_{2}$ Somewhat less likely
$\square$ No change
$\square$ Somewhat more likely
$\square_{5}$ A lot more likely 


\section{About You}

We have a few questions about you so that we may understand the characteristics of our survey respondents. We will keep this information confidential and it is not linked to your name
43. Do you have a current:
Driver's license
Transit pass
Carshare M embership
Yes No

44. How many working motor vehicles does your household own or lease?

(Do not include motorhomes)

$$
\text { \#Vehicles }
$$

(Enter 0 if None)

45. How many working adult bicycles does your household own?

\#Bicycles
$\quad$ (Enter 0 if None)

46. INCLUDING YOURSELF, how many people live in your household?

$$
\begin{aligned}
& \text { \#Adults } \\
& \text { \#Children } \\
& \text { (Enter } 0 \text { if } \overline{\text { if }} \text { None) }
\end{aligned}
$$

47. How long have you lived at this home?

$$
\text { \#Years }
$$

48. Do you rent or own your home?
$\square_{1}$ Rent
$\square_{2}$ Own
$\square_{3}$ Other

49. Do you consider yourself:

(Select all that apply)

$\square$ American Indian or Alaska Native
$\square$ Asian
$\square$ Black or African American
$\square$ Hispanic or Latino/a
$\square$ White or Caucasian
$\square$ Other:

50. What is your age? years

51. What is your gender?
$\square_{1}$ Male
$\square_{2}$ Female

52. What is your employment status? (Select all that apply)

$\square$ I work outside the home-> What is the zip code of your place of work?

$\square$ I go to school outside the home-> What is the zip code of your school?

$\square$ I work from home

$\square$ Not employed at this time

53. What is your annual household income?
$\square_{1}$ Less than $\$ 25,000$
$\square$, $\$ 25,000$ to less than $\$ 50,000$
$\square$ $\square 50,000$ to less than $\$ 75,000$
$\square_{4} \$ 75,000$ to less than $\$ 100,000$
$\square_{5} \$ 100,000$ to less than $\$ 200,000$
$\square_{6} \$ 200,000$ or more
$\square_{9}$ I prefer not to provide this information

54. What is the highest level of school you have completed?
$\square_{1}$ Some high school or less
$\square_{2}$ High school diploma or GED
$\square_{3}$ Some College
$\square_{4}$ Trade/Vocational School
$\square_{5}$ Associate Degree
$\square_{6}$ Four-year college degree or more
$\square_{9}$ Other:

Thank you for taking our survey! If you would like to be entered in a random drawing for one of three \$100 Amazon gift cards please enter your name and contact information on the raffle slip you received with the survey.

55. Is there anything else you'd like to tell us? (Use the space below) 


\section{RESIDENT: AUSTIN - BLUEBONNET}




\section{Neighborhood Street Study}

About How You Get Around
(1) Complete this paper survey and the enclosed raffle slip. Return in the postage-paid envelope;

OR

Complete the online survey and raffle entry at:

http://tinyurl.com/BBStreetStudy

To take the online survey, you will need to enter this code: $\mathbf{B 0 0 0 2}$

Please complete the survey by $7 / 26 / 2013$

\begin{tabular}{|c|c|c|c|c|c|c|}
\hline \multirow{2}{*}{$\begin{array}{l}\text { 1. For each mode of transportation, } \\
\text { please indicate your level of use: }\end{array}$} & \multicolumn{3}{|c|}{$\begin{array}{c}\text { Commute Trips } \\
\text { (to/ from work or school) }\end{array}$} & \multicolumn{3}{|c|}{$\begin{array}{c}\text { Other Trips } \\
\text { (e.g. to the store, park, etc.) }\end{array}$} \\
\hline & Most Trips & Some Trips & No Trips & Most Trips & Some Trips & No Trips \\
\hline Car/truck/motor vehicle (including carpool) & $\square_{1}$ & $\square_{2}$ & $\square_{3}$ & $\square_{1}$ & $\square_{2}$ & $\square_{3}$ \\
\hline Walking & $\square_{1}$ & $\square_{2}$ & $\square_{3}$ & $\square_{1}$ & $\square_{2}$ & $\square_{3}$ \\
\hline Bicycling & $\square_{1}$ & $\square_{2}$ & $\square_{3}$ & $\square_{1}$ & $\square_{2}$ & $\square_{3}$ \\
\hline Public Transportation & $\square_{1}$ & $\square_{2}$ & $\square_{3}$ & $\mathbf{a}_{1}$ & $\square_{2}$ & $\square_{3}$ \\
\hline Other (please specify): & $\square_{1}$ & $\square_{2}$ & $\square_{3}$ & $\square_{1}$ & $\square_{2}$ & $\square_{3}$ \\
\hline
\end{tabular}

2. Compared to two years ago, are you taking more or fewer trips by each mode of transportation?

Car/truck/motor vehicle (including carpool)

Walking

Bicycling

Public Transportation

Other (please specify):

\section{About Your Neighborhood}

3. Over the past two years, changes to my neighborhood as a place for...

\begin{tabular}{|c|c|c|c|c|}
\hline $\begin{array}{c}\text { Very } \\
\text { Negative }\end{array}$ & $\begin{array}{l}\text { Somewhat } \\
\text { Negative }\end{array}$ & $\begin{array}{l}\text { No Impact/ } \\
\text { No Changes }\end{array}$ & $\begin{array}{c}\text { Somewhat } \\
\text { Positive }\end{array}$ & $\begin{array}{c}\text { Very } \\
\text { Positive }\end{array}$ \\
\hline$\square_{1}$ & $\square_{2}$ & $\square_{3}$ & $\square_{4}$ & $\square_{5}$ \\
\hline$\square_{1}$ & $\square_{2}$ & $\square_{3}$ & $\square_{4}$ & $\square_{5}$ \\
\hline$\square_{1}$ & $\square_{2}$ & $\square_{3}$ & $\square_{4}$ & $\square_{5}$ \\
\hline$\square_{1}$ & $\square_{2}$ & $\square_{3}$ & $\square_{4}$ & $\square_{5}$ \\
\hline
\end{tabular}

4. Overall, my level of satisfaction with transportation in my neighborhood is...

walking have been .. driving have been ... .

bicycling have been ...

taking public transportation have been ...

\begin{tabular}{|c|c|c|c|}
\hline $\begin{array}{c}\text { Very } \\
\text { Dissatisfied }\end{array}$ & $\begin{array}{c}\text { Somewhat } \\
\text { Dissatisfied }\end{array}$ & $\begin{array}{c}\text { Somewhat } \\
\text { Satisfied }\end{array}$ & $\begin{array}{c}\text { Very } \\
\text { Satisfied }\end{array}$ \\
\hline$\square_{1}$ & $\square_{2}$ & $\square_{3}$ & $\square_{4}$ \\
\hline
\end{tabular}

About People You Encounter on the Street

5. Please indicate if you agree or disagree with the following statements:

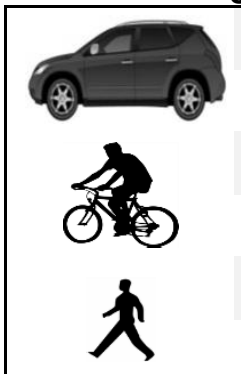

Most drivers follow the rules of the road.

M ost drivers are predictable.

Most bicyclists follow the rules of the road.

Most bicyclists are predictable.

Most pedestrians follow the rules of the road.

M ost pedestrians are predictable. \begin{tabular}{|l|l|l|l|}
\hline Strongly & Somewhat & Somewhat & Strongly \\
No
\end{tabular} Disagree Disagree Agree Agree Opinion

\begin{tabular}{l:c:c:c:c|}
$\square_{1}$ & $\square_{2}$ & $\square_{3}$ & $\square_{4}$ & $\square_{9}$ \\
$\square_{1}$ & $\square_{2}$ & $\square_{3}$ & $\square_{4}$ & $\square_{9}$ \\
$\square_{1}$ & $\square_{2}$ & $\square_{3}$ & $\square_{4}$ & $\square_{9}$ \\
$\square_{1}$ & $\square_{2}$ & $\square_{3}$ & $\square_{4}$ & $\square_{9}$ \\
$\square_{1}$ & $\square_{2}$ & $\square_{3}$ & $\square_{4}$ & $\square_{9}$ \\
$\square_{1}$ & $\square_{2}$ & $\square_{3}$ & $\square_{4}$ & $\square_{9}$ \\
\hline
\end{tabular}




\begin{tabular}{|c|c|c|c|c|c|}
\hline $\begin{array}{l}\text { 6. Please indicate whether you agree or disagree } \\
\text { with the following statements about bicycling: }\end{array}$ & $\begin{array}{l}\text { Strongly } \\
\text { Disagree }\end{array}$ & $\begin{array}{c}\text { Somewhat } \\
\text { Disagree }\end{array}$ & $\begin{array}{c}\text { Somewhat } \\
\text { Agree }\end{array}$ & $\begin{array}{l}\text { Strongly } \\
\text { Agree }\end{array}$ & $\begin{array}{c}\text { No } \\
\text { Opinion }\end{array}$ \\
\hline Bicycling in my city is a convenient way to get places. & $\square_{1}$ & $\square_{2}$ & $\square_{3}$ & $\square_{4}$ & $\square_{9}$ \\
\hline Bicycling in my neighborhood is safe. & $\square_{1}$ & $\square_{2}$ & $\square_{3}$ & $\square_{4}$ & $\square_{9}$ \\
\hline $\begin{array}{l}\text { I am familiar with the bicycle lanes and other bicycle } \\
\text { facilities in my neighborhood. }\end{array}$ & $\square_{1}$ & $\square_{2}$ & $\square_{3}$ & $\square_{4}$ & $\square_{9}$ \\
\hline I would like to bicycle more often for transportation. & $\square_{1}$ & $\square_{2}$ & $\square_{3}$ & $\square_{4}$ & $\square_{9}$ \\
\hline $\begin{array}{l}\text { I would be more likely to ride a bicycle if motor vehicles } \\
\text { and bicycles were physically separated by a barrier. }\end{array}$ & $\square_{1}$ & $\square_{2}$ & $\square_{3}$ & $\square_{4}$ & $\square_{9}$ \\
\hline $\begin{array}{l}\text { Facilities that encourage bicycling for transportation are } \\
\text { a good way to improve public health. }\end{array}$ & $\square_{1}$ & $\square_{2}$ & $\square_{3}$ & $\square_{4}$ & $\square_{9}$ \\
\hline
\end{tabular}

\section{Whether or not you currently ride a bicycle, please consider how comfortable you would be riding a bicycle in each place:}

\begin{tabular}{|c|c|c|c|c|c|c|}
\hline & \begin{tabular}{|c} 
Very \\
Uncomfortable \\
(1)
\end{tabular} & (2) & (3) & (4) & (5) & $\begin{array}{c}\text { Very } \\
\text { Comfortable } \\
\text { (6) }\end{array}$ \\
\hline $\begin{array}{l}\text { (A) On a path or trail } \\
\text { separate from the street }\end{array}$ & $\square_{1}$ & $\square_{2}$ & $\square_{3}$ & $\square_{4}$ & $\square_{5}$ & $\square_{6}$ \\
\hline $\begin{array}{l}\text { (B) On a commercial } \\
\text { street with two lanes of } \\
\text { traffic in each direction, } \\
\text { traffic speeds of } 35 \text { miles } \\
\text { per hour, on-street car } \\
\text { parking, and no bike lane }\end{array}$ & $\square_{1}$ & $\square_{2}$ & $\square_{3}$ & $\square_{4}$ & $\square_{5}$ & $\square_{6}$ \\
\hline $\begin{array}{r}\text { (C) ... On a similar street } \\
\text { to (B), but with a striped } \\
\text { bike lane added }\end{array}$ & $\square_{1}$ & $\square_{2}$ & $\square_{3}$ & $\square_{4}$ & $\square_{5}$ & $\square_{6}$ \\
\hline $\begin{array}{r}\text { (D) ... On a similar } \\
\text { street to (B), but with a } \\
\text { physically separated bike } \\
\text { lane }\end{array}$ & $\square_{1}$ & $\square_{2}$ & $\square_{3}$ & $\square_{4}$ & $\square_{5}$ & $\square_{6}$ \\
\hline
\end{tabular}




\section{Bluebonnet Lane Cycle Track}

A separated two-way bikeway, also known as a "cycle track," was recently built on Bluebonnet Lane from Rabb Glenn Street to Rabb Road. To do this, the parking/ bike lane that existed before was converted to a two-way bicycle lane with a painted "buffer" area and plastic "flexposts" separating the cycle track from the standard traffic lane (see pictures below). The questions on the following pages are related to this cycle track. If you are not familiar with Bluebonnet Lane, please skip to Question 34.

Bluebonnet Lane before and now:
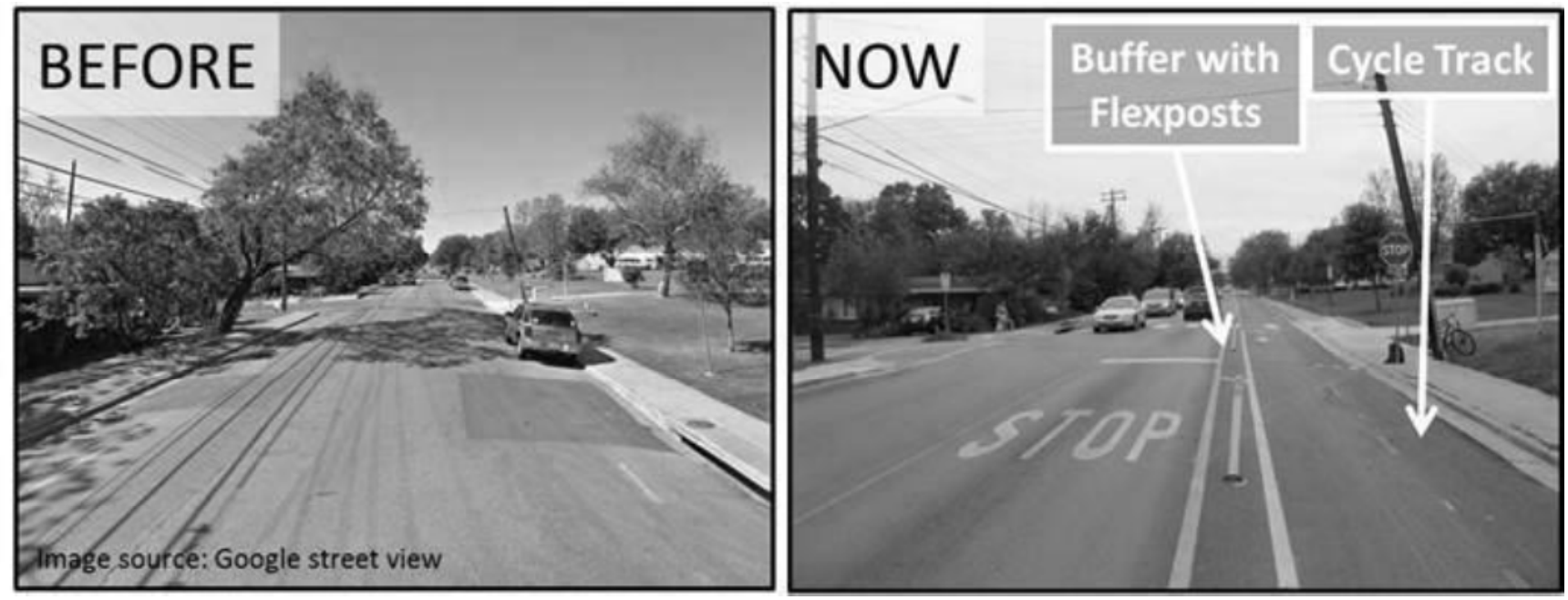

\section{Extent of Bluebonnet Lane cycle track:}

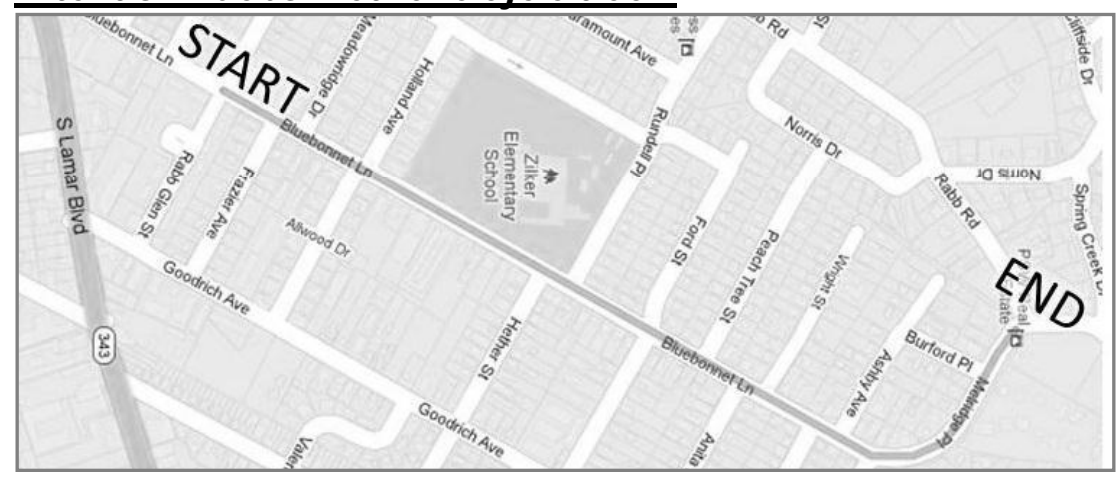

\section{About the Bluebonnet Lane Cycle Track}

8. Because of the Bluebonnet Lane cycle track...

\begin{tabular}{|c|c|c|c|c|c|c|}
\hline & \begin{tabular}{|c}
$\begin{array}{c}\text { Decreased } \\
\text { a Lot }\end{array}$ \\
\end{tabular} & $\begin{array}{l}\text { Decreased } \\
\text { Somewhat }\end{array}$ & $\begin{array}{c}\text { Not } \\
\text { Changed }\end{array}$ & $\begin{array}{l}\text { Increased } \\
\text { Somewhat }\end{array}$ & $\begin{array}{l}\text { Increased } \\
\text { a Lot }\end{array}$ & $\begin{array}{c}\text { No } \\
\text { Opinion }\end{array}$ \\
\hline $\begin{array}{r}\text { the desirability of living in my } \\
\text { neighborhood has... }\end{array}$ & $\square_{1}$ & $\square_{2}$ & $\square_{3}$ & $\square_{4}$ & $\square_{5}$ & $\square_{9}$ \\
\hline the aesthetic appeal of Bluebonnet Lane has... & $\square_{1}$ & $\square_{2}$ & $\square_{3}$ & $\square_{4}$ & $\square_{5}$ & $\square_{9}$ \\
\hline the safety of DRIVING on Bluebonnet Lane has... & $\square_{1}$ & $\square_{2}$ & $\square_{3}$ & $\square_{4}$ & $\square_{5}$ & $\square_{9}$ \\
\hline the safety of BICYCLING on Bluebonnet Lane has... & $\square_{1}$ & $\square_{2}$ & $\square_{3}$ & $\square_{4}$ & $\square_{5}$ & $\square_{9}$ \\
\hline the safety of WALKING on Bluebonnet Lane has... & $\square_{1}$ & $\square_{2}$ & $\square_{3}$ & $\square_{4}$ & $\square_{5}$ & $\square_{9}$ \\
\hline $\begin{array}{r}\text { the number of people I see riding bikes } \\
\text { on Bluebonnet Lane has... }\end{array}$ & $\square_{1}$ & $\square_{2}$ & $\square_{3}$ & $\square_{4}$ & $\square_{5}$ & $\square_{9}$ \\
\hline how well the road works for all people has... & $\square_{1}$ & $\square_{2}$ & $\square_{3}$ & $\square_{4}$ & $\square_{5}$ & $\square_{9}$ \\
\hline
\end{tabular}


About the Bluebonnet Lane Cycle Track, cont.

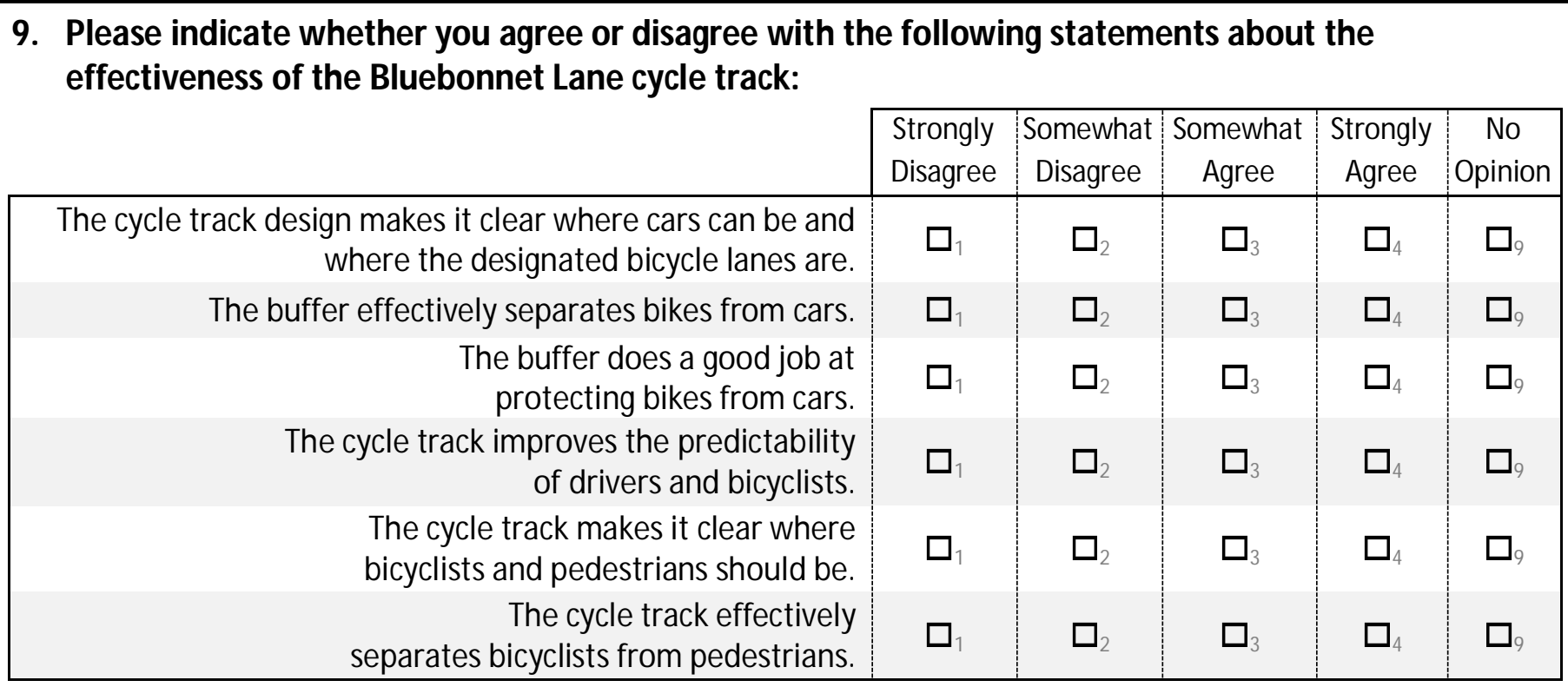

10. Over time, my opinion of the Bluebonnet Lane cycle track has become:

\begin{tabular}{|c|c|c|c|c|}
\hline $\begin{array}{c}\text { A Lot More } \\
\text { Negative }\end{array}$ & $\begin{array}{c}\text { Somewhat } \\
\text { More Negative }\end{array}$ & No Change & $\begin{array}{c}\text { Somewhat } \\
\text { More Positive }\end{array}$ & $\begin{array}{c}\text { A Lot M ore } \\
\text { Positive }\end{array}$ \\
\hline$\square_{1}$ & $\square_{2}$ & $\square_{3}$ & $\square_{4}$ & $\square_{5}$ \\
\hline
\end{tabular}

11. Please indicate whether you agree or disagree with the following statements about cycle tracks.

I would support building more cycle tracks at other locations.

Overall, I support separating bikes from cars.

\begin{tabular}{|c|c|c|c|c|}
\hline $\begin{array}{c}\text { Strongly } \\
\text { Disagree }\end{array}$ & $\begin{array}{c}\text { Somewhat } \\
\text { Disagree }\end{array}$ & $\begin{array}{c}\text { Somewhat } \\
\text { Agree }\end{array}$ & $\begin{array}{c}\text { Strongly } \\
\text { Agree }\end{array}$ & $\begin{array}{c}\text { No } \\
\text { Opinion }\end{array}$ \\
\hline$\square_{1}$ & $\square_{2}$ & $\square_{3}$ & $\square_{4}$ & $\square_{9}$ \\
$\square_{1}$ & $\square_{2}$ & $\square_{3}$ & $\square_{4}$ & $\square_{9}$ \\
\hline
\end{tabular}

\section{About Driving on Bluebonnet Lane}

12. Have you driven a motor vehicle on this section of Bluebonnet Lane since the cycle track was built? $\square_{1}$ Yes (Proceed to Question 13) $\square_{0}$ No (Skip to Question 17)

\section{Since the Bluebonnet Lane cycle track was} built ...

the number of bicyclists riding in the same lanes with cars on Bluebonnet Lane has... how safe and predictable bicyclists are acting has ... how safe and predictable drivers are acting has ... the amount of time it takes me to drive on Bluebonnet Lane has... \begin{tabular}{|l|l|l|l|l|}
\hline Decreased Decreased Not Increased Increased No \\
\hline
\end{tabular} a Lot Somewhat Changed Somewhat a Lot Opinion

\begin{tabular}{l|l:l}
$\square_{1}$ & $\square_{2}$ & \\
$\square_{1}$ & $\square_{2}$ & \\
$\square_{1}$ & $\square_{2}$ & \\
$\square_{1}$ & $\square_{2}$ &
\end{tabular}

$\square_{3}$

$\square_{4}$

$\square_{5}$

$\square_{9}$

口

$\square$

$\square$

$\square_{4}$

$\square_{3}$ 
About Driving on Bluebonnet Lane, cont.

\begin{tabular}{|c|c|c|c|c|c|}
\hline $\begin{array}{l}\text { 14. The impact of the Bluebonnet Lane cycle track } \\
\text { on .... }\end{array}$ & $\begin{array}{l}\text { Very } \\
\text { Negative }\end{array}$ & $\begin{array}{l}\text { Somewhat } \\
\text { Negative }\end{array}$ & $\begin{array}{l}\text { No Impact/ } \\
\text { Neutral }\end{array}$ & $\begin{array}{l}\text { Somewhat } \\
\text { Positive }\end{array}$ & $\begin{array}{l}\text { Very } \\
\text { Positive }\end{array}$ \\
\hline traffic congestion has been ... & $\square_{1}$ & $\square_{2}$ & $\square_{3}$ & $\square_{4}$ & $\square_{5}$ \\
\hline $\begin{array}{r}\text { my ability to turn off of Bluebonnet Lane into side } \\
\text { streets and driveways has been ... }\end{array}$ & $\square_{1}$ & $\square_{2}$ & $\square_{3}$ & $\square_{4}$ & $\square_{5}$ \\
\hline $\begin{array}{r}\text { my ability to pull onto Bluebonnet Lane from side } \\
\text { streets and driveways has been ... }\end{array}$ & $\square_{1}$ & $\square_{2}$ & $\square_{3}$ & $\square_{4}$ & $\square_{5}$ \\
\hline $\begin{array}{r}\text { my ability to find a parking spot on } \\
\text { Bluebonnet Lane has been ... }\end{array}$ & $\square_{1}$ & $\square_{2}$ & $\square_{3}$ & $\square_{4}$ & $\square_{5}$ \\
\hline $\begin{array}{l}\text { how stressful it is to park a car on } \\
\text { Bluebonnet Lane has been ... }\end{array}$ & $\square_{1}$ & $\square_{2}$ & $\square_{3}$ & $\square_{4}$ & $\square_{5}$ \\
\hline
\end{tabular}

\section{Please indicate if you agree or disagree with the following statements about turning across the cycle track}

When I want to make a left turn (across the cycle track), I am able to adequately see if there are any approaching bicyclists in the cycle track.

When I want to make a right turn (across the cycle track), I am able to adequately see if there are any approaching bicyclists in the cycle track.

\begin{tabular}{|c|c|c|c|c|}
\hline $\begin{array}{c}\text { Strongly } \\
\text { Disagree }\end{array}$ & $\begin{array}{c}\text { Somewhat } \\
\text { Disagree }\end{array}$ & $\begin{array}{c}\text { Somewhat } \\
\text { Agree }\end{array}$ & $\begin{array}{c}\text { Strongly } \\
\text { Agree }\end{array}$ & $\begin{array}{c}\text { No } \\
\text { Opinion }\end{array}$ \\
$\square_{1}$ & $\square_{2}$ & $\square_{3}$ & $\square_{4}$ & $\square_{9}$ \\
$\square_{1}$ & $\square_{2}$ & $\square_{3}$ & $\square_{4}$ & $\square_{9}$ \\
\hline
\end{tabular}

16. Do you avoid driving on Bluebonnet Lane because of the cycle track?
$\square_{1}$ Yes
No

\section{About Bicycling on the Bluebonnet Lane Cycle Track}

17. Have you bicycled on this section of Bluebonnet Lane since the cycle track was built?
Yes (Proceed to Question 18)
$\square_{0}$ No (Skip to Question 23)

18. How comfortable do you feel bicycling in the Bluebonnet Lane cycle track...

in the lane closest to the sidewalk (i.e. southbound)?

in the lane closest to the standard traffic lanes and plastic flexposts (i.e. northbound)?

\begin{tabular}{|c|c|c|c|c|c|}
\hline $\begin{array}{c}\text { Very } \\
\text { Uncomfortable } \\
(1)\end{array}$ & (2) & (3) & (4) & (5) & $\begin{array}{c}\text { Very } \\
\text { Comfortable } \\
(6)\end{array}$ \\
\hline$\square_{1}$ & $\square_{2}$ & $\square_{3}$ & $\square_{4}$ & $\square_{5}$ & $\square_{6}$ \\
$\square_{1}$ & $\square_{2}$ & $\square_{3}$ & $\square_{4}$ & $\square_{5}$ & $\square_{6}$ \\
\hline
\end{tabular}

19. When bicycling on Bluebonnet Lane, how often do you ride on the following... .

Cycle track

Standard traffic lanes

Sidewalk

\begin{tabular}{|c|c|c|c|c|}
\hline & Always & $\begin{array}{c}\text { Most of the } \\
\text { time }\end{array}$ & $\begin{array}{c}\text { Some of the } \\
\text { time }\end{array}$ & Never \\
\hline & $\square_{1}$ & $\square_{2}$ & $\square_{3}$ & $\square_{4}$ \\
\hline & $\square_{1}$ & $\square_{2}$ & $\square_{3}$ & $\square_{4}$ \\
\hline$\square_{1}$ & $\square_{2}$ & $\square_{3}$ & $\square_{4}$ \\
\hline
\end{tabular}


About Bicycling on the Bluebonnet Lane Cycle Track, cont.

\begin{tabular}{|c|c|c|c|c|c|c|}
\hline $\begin{array}{l}\text { 20. Because of the Bluebonnet Lane cycle track } \\
\text {. . . }\end{array}$ & $\begin{array}{l}\text { Decreased } \\
\text { a Lot }\end{array}$ & $\begin{array}{l}\text { Decreased } \\
\text { Somewhat }\end{array}$ & $\begin{array}{c}\text { Not } \\
\text { Changed }\end{array}$ & $\begin{array}{l}\text { Increased } \\
\text { Somewhat }\end{array}$ & $\begin{array}{c}\text { Increased } \\
\text { a Lot }\end{array}$ & $\begin{array}{c}\text { No } \\
\text { Opinion }\end{array}$ \\
\hline $\begin{array}{l}\text { the likelihood that I will choose to bicycle on } \\
\text { Bluebonnet as opposed to other streets has... }\end{array}$ & $\square_{1}$ & $\square_{2}$ & $\square_{3}$ & $\square_{4}$ & $\square_{5}$ & $\square_{9}$ \\
\hline the time it takes me to bicycle on Bluebonnet has... & $\square_{1}$ & $\square_{2}$ & $\square_{3}$ & $\square_{4}$ & $\square_{5}$ & $\square_{9}$ \\
\hline $\begin{array}{l}\text { the usefulness of Bluebonnet Lane for getting places } \\
\qquad \text { I want to go has... }\end{array}$ & $\square_{1}$ & $\square_{2}$ & $\square_{3}$ & $\square_{4}$ & $\square_{5}$ & $\square_{9}$ \\
\hline drivers' awareness of bicyclists on Bluebonnet has... & $\square_{1}$ & $\square_{2}$ & $\square_{3}$ & $\square_{4}$ & $\square_{5}$ & $\square_{9}$ \\
\hline drivers' speeds on Bluebonnet Lane have... & $\square_{1}$ & $\square_{2}$ & $\square_{3}$ & $\square_{4}$ & $\square_{5}$ & $\square_{9}$ \\
\hline $\begin{array}{l}\text { how comfortable I feel when bicycling } \\
\text { on Bluebonnet Lane has... }\end{array}$ & $\square_{1}$ & $\square_{2}$ & $\square_{3}$ & $\square_{4}$ & $\square_{5}$ & $\square_{9}$ \\
\hline how often I ride a bicycle overall has... & $\square_{1}$ & $\square_{2}$ & $\square_{3}$ & $\square_{4}$ & $\square_{5}$ & $\square_{9}$ \\
\hline $\begin{array}{l}\text { the difficulty of turning across Bluebonnet Lane } \\
\text { while bicycling has... }\end{array}$ & $\square_{1}$ & $\square_{2}$ & $\square_{3}$ & $\square_{4}$ & $\square_{5}$ & $\square_{9}$ \\
\hline $\begin{array}{r}\text { the difficulty of navigating around } \\
\text { pedestrians has... }\end{array}$ & $\square_{1}$ & $\square_{2}$ & $\square_{3}$ & $\square_{4}$ & $\square_{5}$ & $\square_{9}$ \\
\hline how comfortable I feel bicycling with my kids has... & $\square_{1}$ & $\square_{2}$ & $\square_{3}$ & $\square_{4}$ & $\square_{5}$ & $\square_{9}$ \\
\hline how safe I feel bicycling with my kids has. & $\square_{1}$ & $\square_{2}$ & $\square_{3}$ & $\square_{4}$ & $\square_{5}$ & $\square_{9}$ \\
\hline
\end{tabular}

21. In the past 30 days, how many days have you bicycled for transportation (i.e. not just for recreation/ exercise)?

22. Did you fill out a separate online bicyclist survey about the Bluebonnet Lane cycle track from us recently?
$\square_{1}$ Yes
No

\section{About Walking on Bluebonnet Lane}

23. Have you walked on this section of Bluebonnet Lane since the cycle track was built?

$\square_{1}$ Yes (Proceed to Question 24) $\quad \square_{0}$ No (Skip to Question 27)

24. How often do bicyclists in the cycle track stop for pedestrians at crosswalks?

\begin{tabular}{|c|c|c|c|}
\hline Usually & Sometimes & Rarely & Never \\
\hline$\square_{1}$ & $\square_{2}$ & $\square_{3}$ & $\square_{4}$ \\
\hline
\end{tabular}

\section{Because of the Bluebonnet Lane cycle} track...

$$
\begin{array}{r}
\text { drivers' speeds on Bluebonnet Lane } \\
\text { have generally ... } \\
\text { the number of bicyclists riding } \\
\text { on the sidewalk has... }
\end{array}
$$
my satisfaction with the walking environment on Bluebonnet Lane has... my sense of safety when crossing Bluebonnet Lane has...

\begin{tabular}{|c|c|c|c|c|c|}
\hline $\begin{array}{c}\text { Decreased } \\
\text { a Lot }\end{array}$ & $\begin{array}{c}\text { Decreased } \\
\text { Somewhat }\end{array}$ & $\begin{array}{c}\text { Not } \\
\text { Changed }\end{array}$ & $\begin{array}{c}\text { Increased } \\
\text { Somewhat }\end{array}$ & $\begin{array}{c}\text { Increased } \\
\text { a Lot }\end{array}$ & $\begin{array}{c}\text { No } \\
\text { Opinion }\end{array}$ \\
\hline$\square_{1}$ & $\square_{2}$ & $\square_{3}$ & $\square_{4}$ & $\square_{5}$ & $\square_{9}$ \\
$\square_{1}$ & $\square_{2}$ & $\square_{3}$ & $\square_{4}$ & $\square_{5}$ & $\square_{9}$ \\
$\square_{1}$ & $\square_{2}$ & $\square_{3}$ & $\square_{4}$ & $\square_{5}$ & $\square_{9}$ \\
$\square_{1}$ & $\square_{2}$ & $\square_{3}$ & $\square_{4}$ & $\square_{5}$ & $\square_{9}$ \\
\hline
\end{tabular}


About Walking on Bluebonnet Lane, cont.

26. Do you ever walk or jog in the cycle track, rather than on the sidewalk?

$\square_{0}$ No
$\square_{1}$ Yes (Please explain why you choose the cycle track

over the sidewalk in the space to the right) $\rightarrow$

\section{About Zilker Elementary School}

27. Do you have a child (or children) that attends Zilker Elementary School?

$\square_{0}$ No (Skip to Question 32)

$\square_{1}$ Yes (If yes, what age(s)) $\rightarrow$

28. We would like to know about if your child bikes or walks to or from Zlker Elementary School.

\begin{tabular}{|c|c|c|c|c|c|c|}
\hline \multirow[b]{2}{*}{ My child or children . . . } & \multicolumn{3}{|c|}{$\begin{array}{l}\text { How often do your children } \\
\text { bike or walk to school? }\end{array}$} & \multicolumn{3}{|c|}{$\begin{array}{c}\text { Do they bike or walk ... } \\
\text { (Select all that apply) }\end{array}$} \\
\hline & Never & $\begin{array}{c}\text { Some of the } \\
\text { time }\end{array}$ & $\begin{array}{l}\text { Most of the } \\
\text { time }\end{array}$ & $\begin{array}{c}\text { With an } \\
\text { adult }\end{array}$ & $\begin{array}{l}\text { With other } \\
\text { children }\end{array}$ & Alone \\
\hline bike(s) to school ... & $\square_{1}$ & $\square_{2}$ & $\square_{3}$ & $\square_{1}$ & $\square_{2}$ & $\square_{3}$ \\
\hline walk(s) to school ... & $\square_{1}$ & $\square_{2}$ & $\square_{3}$ & $\square_{1}$ & $\square_{2}$ & $\square_{3}$ \\
\hline
\end{tabular}

29. Does your child or children bike or walk on Bluebonnet Lane?
$\square_{1}$ Yes, bikes
2 Yes, walks
Yes, walks and bikes
$\square$ No

30. Please indicate to what degree these issues are barriers that prevent your child(ren) from biking or walking to school:

\begin{tabular}{|r|c|c|c|c|c|}
\cline { 2 - 6 } & \multicolumn{5}{c|}{ Biking } \\
\cline { 2 - 6 } & $\begin{array}{c}\text { (1) Not a } \\
\text { barrier at all }\end{array}$ & (2) & (3) & (4) & Barrier \\
\hline Distance is too great & $\square_{1}$ & $\square_{2}$ & $\square_{3}$ & $\square_{4}$ & $\square_{5}$ \\
Stranger danger & $\square_{1}$ & $\square_{2}$ & $\square_{3}$ & $\square_{4}$ & $\square_{5}$ \\
Traffic & $\square_{1}$ & $\square_{2}$ & $\square_{3}$ & $\square_{4}$ & $\square_{5}$ \\
$\begin{array}{r}\text { Not enough time } \\
\text { Child is not interested } \\
\text { in this activity }\end{array}$ & $\square_{1}$ & $\square_{2}$ & $\square_{3}$ & $\square_{4}$ & $\square_{5}$ \\
Other (please specify): & $\square_{1}$ & $\square_{2}$ & $\square_{3}$ & $\square_{4}$ & $\square_{5}$ \\
\hline
\end{tabular}

\begin{tabular}{|c|c|c|c|c|}
\hline \multicolumn{5}{|c|}{ Walking } \\
\hline $\begin{array}{c}\text { (1) Not a } \\
\text { barrier at all }\end{array}$ & (2) & (3) & (4) & $\begin{array}{c}\text { (5) M ajor } \\
\text { Barrier }\end{array}$ \\
\hline$\square_{1}$ & $\square_{2}$ & $\square_{3}$ & $\square_{4}$ & $\square_{5}$ \\
\hline$\square_{1}$ & $\square_{2}$ & $\square_{3}$ & $\square_{4}$ & $\square_{5}$ \\
\hline$\square_{1}$ & $\square_{2}$ & $\square_{3}$ & $\square_{4}$ & $\square_{5}$ \\
\hline$\square_{1}$ & $\square_{2}$ & $\square_{3}$ & $\square_{4}$ & $\square_{5}$ \\
\hline$\square_{1}$ & $\square_{2}$ & $\square_{3}$ & $\square_{4}$ & $\square_{5}$ \\
\hline$\square_{1}$ & $\square_{2}$ & $\square_{3}$ & $\square_{4}$ & $\square_{5}$ \\
\hline
\end{tabular}

31. Please indicate your level of agreement with the following:

Since the cycle track on Bluebonnet Lane was built:

\begin{tabular}{|c|c|c|c|c|c|}
\hline built: & Disagree & Disagree & Agree & Agree & Opinion \\
\hline My children are biking to school more often. & $\square_{1}$ & $\square_{2}$ & $\square_{3}$ & $\square_{4}$ & $\square_{9}$ \\
\hline My children are walking to school more often. & $\square_{1}$ & $\square_{2}$ & $\square_{3}$ & $\square_{4}$ & $\square_{9}$ \\
\hline Biking on Bluebonnet Lane is safer for children now. & $\square_{1}$ & $\square_{2}$ & $\square_{3}$ & $\square_{4}$ & $\square_{9}$ \\
\hline Walking on Bluebonnet Lane is safer for children now. & $\square_{1}$ & $\square_{2}$ & $\square_{3}$ & $\square_{4}$ & $\square_{9}$ \\
\hline $\begin{array}{l}\text { The cycle track is an important reason for why I allow } \\
\text { my child to bike on Bluebonnet Lane. }\end{array}$ & $\square_{1}$ & $\square_{2}$ & $\square_{3}$ & $\square_{4}$ & $\square_{9}$ \\
\hline $\begin{array}{l}\text { The cycle track is an important reason for why I allow } \\
\text { my child to walk on Bluebonnet Lane. }\end{array}$ & $\square_{1}$ & $\square_{2}$ & $\square_{3}$ & $\square_{4}$ & $\square_{9}$ \\
\hline
\end{tabular}




\section{BARTON SPRINGS ROAD}

34. Barton Springs Road has also experienced some changes recently. Would you be willing to answer a few similar questions about these changes, too?

$\square_{1}$ Yes (Proceed to Question 35) $\square_{0}$ No (Skip to Question 54 on the last page of the questionnaire)

\section{Barton Springs Road Cycle Track}

A separated one-way eastbound bikeway, also known as a "cycle track," was recently built on Barton Springs Road from Lamar Boulevard to 1st Street. The cycle track includes a painted "buffer" area and plastic "flexposts" separating the cycle track from the standard traffic lane (see pictures below). The questions on the following pages are related to this cycle track.

Barton Springs Road before and now:
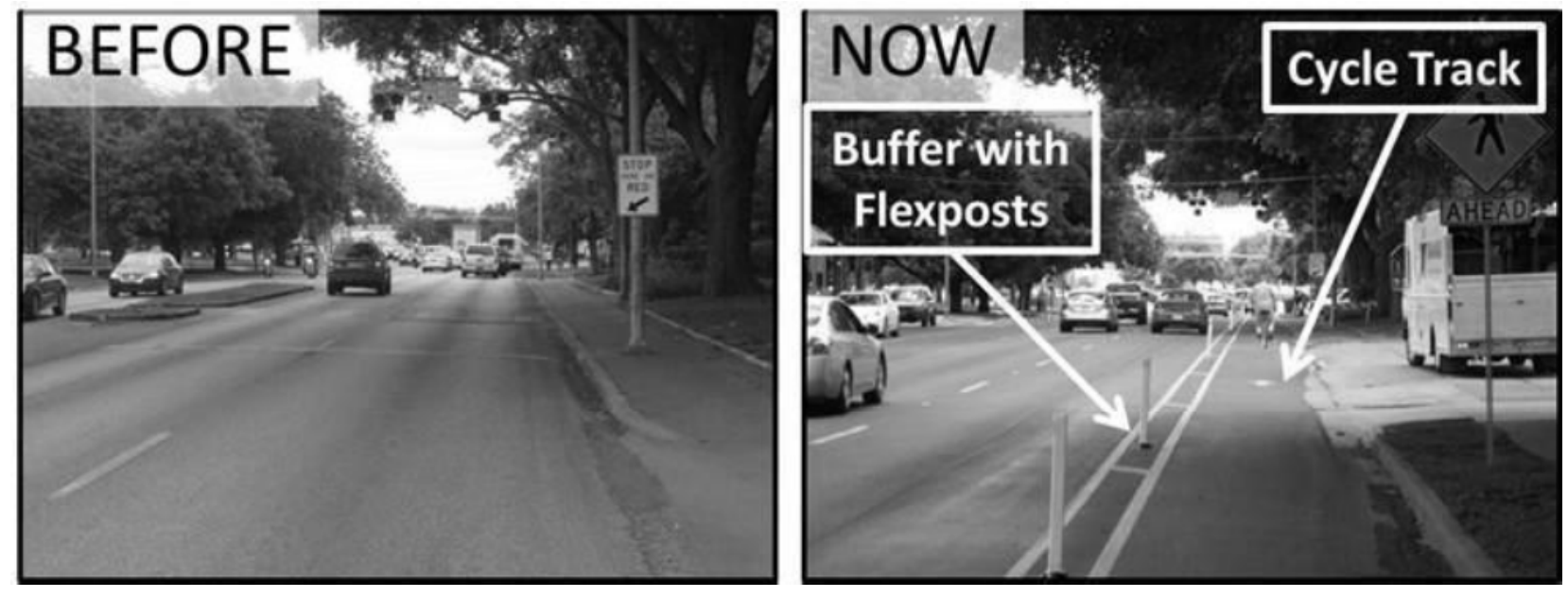

Extent of Barton Springs Road cycle track:

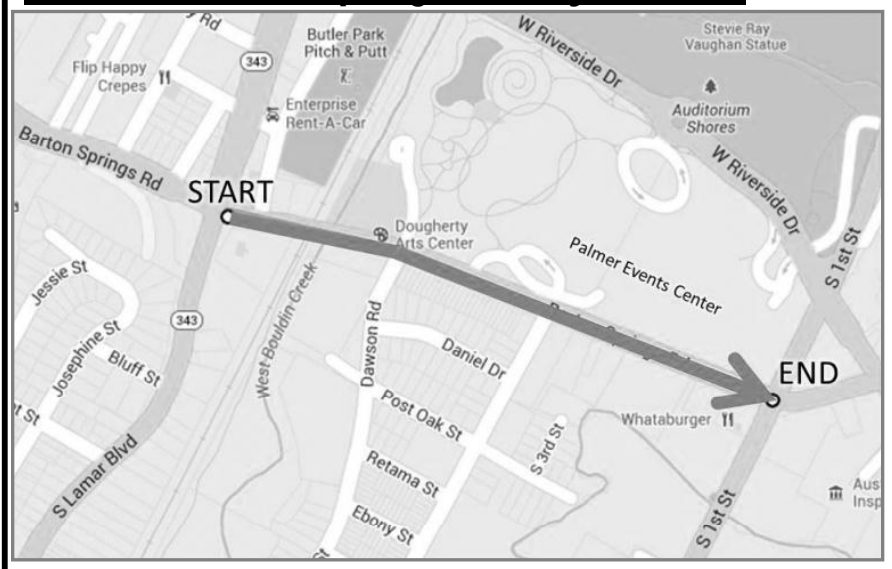


About the Barton Springs Road Cycle Track

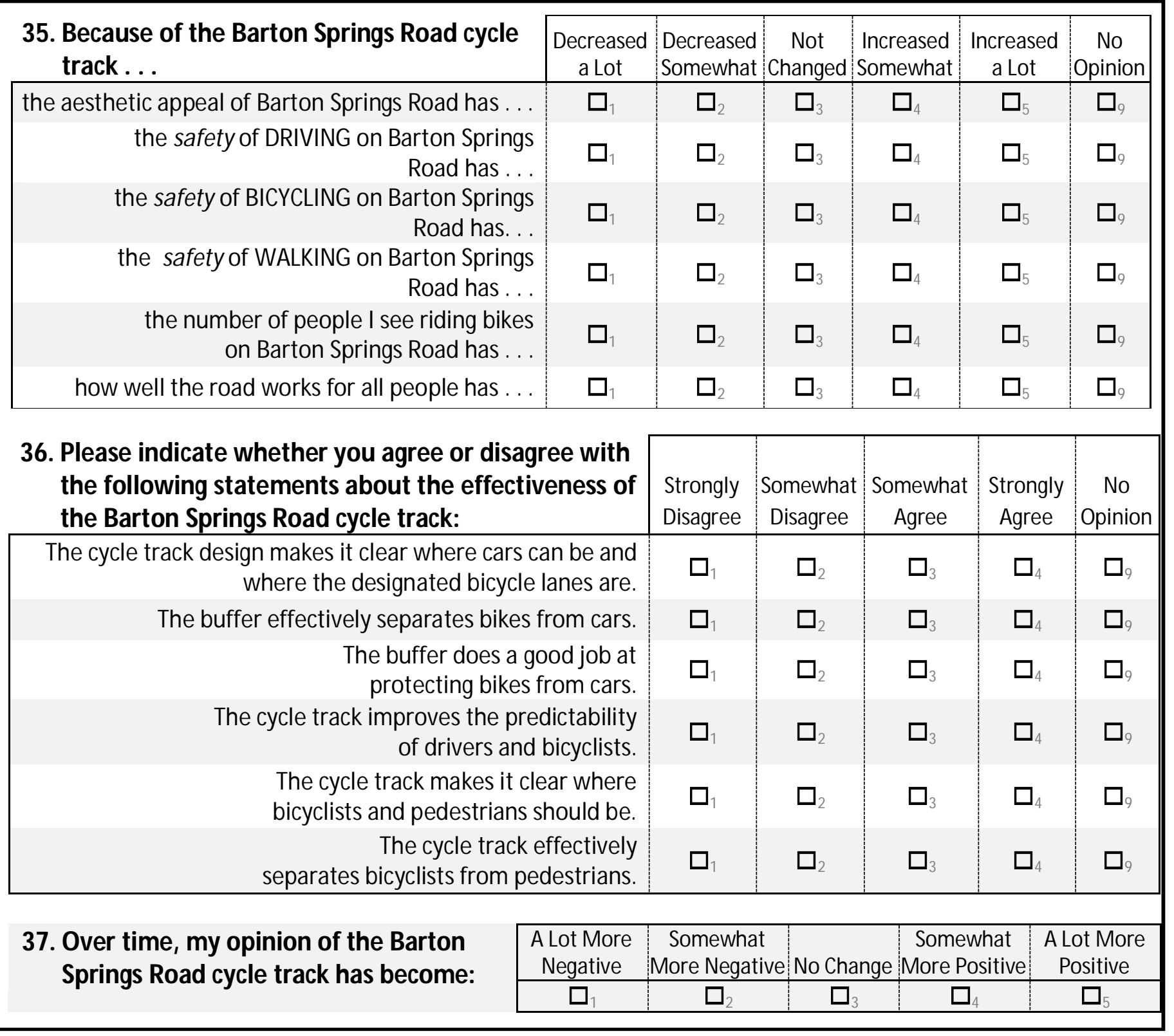

\section{About Driving on Barton Springs Road}

38. Have you driven a motor vehicle eastbound (toward 1st Street and Congress Avenue) on this section of Barton Springs Road since the cycle track was built?
$\square_{1}$ Yes (Proceed to Question 39)
$\square_{0}$ No (Skip to Question 43)

39. Since the Barton Springs Road cycle track was built ...

the number of bicyclists riding in the same lanes with cars on Barton Springs Road has... how safe and predictable bicyclists are acting has ... how safe and predictable drivers are acting has ... the amount of time it takes me to drive on Barton Springs Road has...

Decreased Decreased Not Increased Increased No a Lot Somewhat Changed Somewhat a Lot Opinion

\begin{tabular}{l|l|l}
$\square_{1}$ & $\square_{2}$ & $\square_{3}$ \\
$\square_{1}$ & $\square_{2}$ & $\square_{3}$ \\
$\square_{1}$ & $\square_{2}$ & $\square_{3}$ \\
$\square_{1}$ & $\square_{2}$ & $\square_{3}$
\end{tabular}

$\square_{4}$
$\square_{4}$
$\square_{4}$
$\square_{4}$

$\square_{5}$
$\square_{5}$
$\square_{5}$
$\square_{5}$

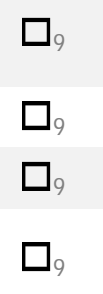


About Driving on Barton Springs Road, cont.

\begin{tabular}{|c|c|c|c|c|c|}
\hline $\begin{array}{l}\text { 40. The impact of the Barton Springs Road cycle } \\
\text { track on . . . }\end{array}$ & $\begin{array}{c}\text { Very } \\
\text { Negative }\end{array}$ & $\begin{array}{l}\text { Somewhat } \\
\text { Negative }\end{array}$ & $\begin{array}{l}\text { No Impact/ } \\
\text { Neutral }\end{array}$ & $\begin{array}{l}\text { Somewhat } \\
\text { Positive }\end{array}$ & $\begin{array}{l}\text { Very } \\
\text { Positive }\end{array}$ \\
\hline traffic congestion has been ... & $\square_{1}$ & $\square_{2}$ & $\square_{3}$ & $\square_{4}$ & $\square_{5}$ \\
\hline $\begin{array}{r}\text { my ability to turn off of Barton Springs Road at signalized } \\
\text { intersections has been ... }\end{array}$ & $\square_{1}$ & $\square_{2}$ & $\square_{3}$ & $\square_{4}$ & $\square_{5}$ \\
\hline $\begin{array}{r}\text { my ability to turn off of Barton Springs Road into } \\
\text { driveways has been ... }\end{array}$ & $\square_{1}$ & $\square_{2}$ & $\square_{3}$ & $\square_{4}$ & $\square_{5}$ \\
\hline $\begin{array}{r}\text { my ability to pull onto Barton Springs Road from } \\
\text { driveways has been ... }\end{array}$ & $\square_{1}$ & $\square_{2}$ & $\square_{3}$ & $\square_{4}$ & $\square_{5}$ \\
\hline
\end{tabular}

\section{Please indicate if you agree or disagree with the following statement.}

When I want to make a right turn (across the cycle track), I am able to adequately see if there are any approaching bicyclists in the cycle track.

\begin{tabular}{|c|c|c|c|c|}
\hline $\begin{array}{c}\text { Strongly } \\
\text { Disagree }\end{array}$ & $\begin{array}{c}\text { Somewhat } \\
\text { Disagree }\end{array}$ & $\begin{array}{c}\text { Somewhat } \\
\text { Agree }\end{array}$ & $\begin{array}{c}\text { Strongly } \\
\text { Agree }\end{array}$ & $\begin{array}{c}\text { No } \\
\text { Opinion }\end{array}$ \\
\hdashline$\square_{1}$ & $\square_{2}$ & $\square_{3}$ & $\square_{4}$ & $\square_{9}$ \\
\hline
\end{tabular}

42. Do you avoid driving on Barton Springs Road because of the cycle track?

$\square_{1}$ Yes

$\square_{0}$ No

\section{About Bicycling on the Barton Springs Road Cycle Track}

43. Have you bicycled eastbound (toward 1st Street and Congress Avenue) on this section of Barton Springs Road since the cycle track was built?

$\square_{1}$ Yes (Proceed to Question 44) $\square_{0}$ No (Skip to Question 49)

\section{How comfortable do you feel when bicycling in the Barton Springs Road cycle track?}

\begin{tabular}{|c|c|c|c|c|c|}
\hline $\begin{array}{c}\text { Very } \\
\text { Uncomfortable } \\
(1)\end{array}$ & $(2)$ & $(3)$ & $(4)$ & $(5)$ & $\begin{array}{c}\text { Very } \\
\text { Comfortable } \\
(6)\end{array}$ \\
\hline$\square_{1}$ & $\square_{2}$ & $\square_{3}$ & $\square_{4}$ & $\square_{5}$ & $\square_{6}$ \\
\hline
\end{tabular}

\section{Because of the Barton Springs Road cycle} track ...

the likelihood that I will choose to bicycle on

Barton Springs as opposed to other streets has...

the time it takes me to bicycle on Barton Springs has... the usefulness of Barton Springs Road for getting places I want to go has...

drivers' awareness of bicyclists on Barton Springs has...

drivers' speeds on Barton Springs Road have ...

how comfortable I feel when bicycling on Barton Springs Road has ...

how often I ride a bicycle overall has...

the difficulty of turning left from Barton Springs Road while bicycling has...

the difficulty of navigating around turning motor vehicles has...

the difficulty of navigating around pedestrians has ... how comfortable I feel bicycling with my kids has... how safe I feel bicycling with my kids has ... \begin{tabular}{|l|l|l|l|l|l}
\hline Decreased & Decreased & Not & Increased & Increased & No
\end{tabular} a Lot Somewhat Changed Somewhat a Lot Opinion 
About Bicycling on the Barton Springs Road Cycle Track, cont.

\begin{tabular}{|c|c|c|c|c|}
\hline $\begin{array}{l}\text { 46. When bicycling EASTBOUND (toward 1st Street } \\
\text { and Congress Avenue) on Barton Springs Road, } \\
\text { how often do you ride on the following... }\end{array}$ & Always & $\begin{array}{l}\text { Most of the } \\
\text { time }\end{array}$ & $\begin{array}{l}\text { Some of } \\
\text { the time }\end{array}$ & Never \\
\hline $\begin{array}{ll}\text { Cycle track } \\
\end{array}$ & $\square_{1}$ & $\square_{2}$ & $\square_{3}$ & $\square_{4}$ \\
\hline Standard traffic lanes & $\mathbf{\square}_{1}$ & $\square_{2}$ & $\square_{3}$ & $\square_{4}$ \\
\hline $\begin{array}{rr}\text { Sidewalk } \\
\end{array}$ & $\mathbf{\square}_{1}$ & $\square_{2}$ & $\square_{3}$ & $\square_{4}$ \\
\hline Shared-use path on north side of Barton Springs Road & $\mathbf{\square}_{1}$ & $\square_{2}$ & $\square_{3}$ & $\square_{4}$ \\
\hline
\end{tabular}

47. In the past 30 days, how many days have you bicycled for transportation (i.e. not just for recreation/ exercise)?

48. Did you fill out a separate online bicyclist survey about the Barton Springs Road cycle track from us recently?
$\square_{1}$ Yes
$\square$ No

\section{About Walking on Barton Springs Road}

49. Have you walked on the south side of this section of Barton Springs Road since the cycle track was built?

$\square_{1}$ Yes (Proceed to Question 50) $\quad \square_{0}$ No (Skip to Question 52)

50. Because of the Barton Springs Road cycle track...

drivers' speeds on Barton Springs Road have generally ...

the number of bicyclists riding on the sidewalk has...

my satisfaction with the walking environment on Barton Springs Road has... my sense of safety when crossing Barton Springs Road has...

\begin{tabular}{|c|c|c|c|c|c|}
\hline $\begin{array}{c}\text { Decreased } \\
\text { a Lot }\end{array}$ & $\begin{array}{c}\text { Decreased } \\
\text { Somewhat }\end{array}$ & $\begin{array}{c}\text { Not } \\
\text { Changed }\end{array}$ & $\begin{array}{l}\text { Increased } \\
\text { Somewhat }\end{array}$ & $\begin{array}{c}\text { Increased } \\
\text { a Lot }\end{array}$ & $\begin{array}{c}\text { No } \\
\text { Opinion }\end{array}$ \\
\hline$\square_{1}$ & $\square_{2}$ & $\square_{3}$ & $\square_{4}$ & $\square_{5}$ & $\square_{9}$ \\
$\square_{1}$ & $\square_{2}$ & $\square_{3}$ & $\square_{4}$ & $\square_{5}$ & $\square_{9}$ \\
$\square_{1}$ & $\square_{2}$ & $\square_{3}$ & $\square_{4}$ & $\square_{5}$ & $\square_{9}$ \\
$\square_{1}$ & $\square_{2}$ & $\square_{3}$ & $\square_{4}$ & $\square_{5}$ & $\square_{9}$ \\
\hline
\end{tabular}

51. Do you ever walk or jog in the cycle track, rather than on the sidewalk?

$\square_{0}$ No

$\square_{1}$ Yes (Please explain why you choose the cycle track over the sidewalk in the space to the right) $\rightarrow$

Overall Impressions of the Barton Springs Road Cycle Track

52. What is the best thing about the cycle track?

53. What is the worst thing about the cycle track? 
We have a few questions about you so that we may understand the characteristics of our survey respondents. We will keep this information confidential and it is not linked to your name

$\begin{array}{ccc}\text { 54. Do you have a current: } & \text { Yes } & \text { No } \\ \text { Driver's license } & \square_{1} & \square_{0} \\ \text { Transit pass } & \square_{1} & \square_{0} \\ \text { Carshare M embership } & \square_{1} & \square_{0}\end{array}$

55. How many working motor vehicles does your household own or lease?

(Do not include motorhomes)

$$
\text { \#Vehicles }
$$

(Enter $\overline{0}$ if None)

56. How many working adult bicycles does your household own?

$$
\begin{aligned}
& \text { \# Bicycles } \\
& \text { (Enter o } \mathrm{if} \text { None-e) }
\end{aligned}
$$

57. INCLUDING YOURSELF, how many people live in your household?

$$
\begin{aligned}
& \text { \#Adults } \\
& \text { \#Children } \\
& \text { (Enter ōif } \overline{-} \text { None-) }
\end{aligned}
$$

58. How long have you lived at this home?

$$
\text { \#Years }
$$

59. Do you rent or own your home?
$\square_{1}$ Rent
$\square_{2}$ Own
$\square_{3}$ Other

60. Do you consider yourself:

(Select all that apply)

$\square$ American Indian or Alaska Native
$\square$ Asian
$\square$ Black or African American
$\square$ Hispanic or Latino/a
$\square$ White or Caucasian
$\square$ Other:

61. What is your age? years

62. What is your gender?

$\square_{1}$ Male $\square_{2}$ Female

63. What is your employment status?

(Select all that apply)

$\square$ I work outside the home-> What is the zip code of your place of work?

$\square$ I go to school outside the home-> What is the zip code of your school?
$\square$ I work from home
$\square$ Not employed at this time

64. What is your annual household income?
$\square_{1}$ Less than $\$ 25,000$
$\square_{2} \$ 25,000$ to less than $\$ 50,000$
$\square_{3} \$ 50,000$ to less than $\$ 75,000$
$\square_{4} \$ 75,000$ to less than $\$ 100,000$
$\square_{5} \$ 100,000$ to less than $\$ 200,000$
$\square_{6} \$ 200,000$ or more
$\square_{9}$ I prefer not to provide this information

65. What is the highest level of school you have completed?
$\square_{1}$ Some high school or less
$\square_{2}$ High school diploma or GED
$\square_{3}$ Some College
$\square_{4}$ Trade/Vocational School
$\square$ Associate Degree
$\square_{6}$ Four-year college degree or more
$\square_{9}$ Other:

Thank you for taking our survey! If you would like to be entered in a random drawing for one of three \$100 Amazon gift cards please enter your name and contact information on the raffle slip you received with the survey.

66. Is there anything else you'd like to tell us? (Use the space below) 


\section{Resident: Chicago - Milwaukee Ave.}




\section{Neighborhood Street Study}

\section{About How You Get Around}

1. For each mode of transportation, please indicate your level of use:

Car/truck/motor vehicle (including carpool)
Walking
Bicycling

Other (please specify):
(1) Complete this paper survey and the enclosed raffle slip. Return in the postage-paid envelope;

OR

(2) Complete the online survey and raffle entry at: http:// tinyurl.com/M ilwAve To take the online survey, you will need to enter this code: $\mathbf{M 0 0 0 1 8}$

Survey deadline 10/17/ 2013

2. Compared to two years ago, are you taking more or fewer trips by each mode of transportation?

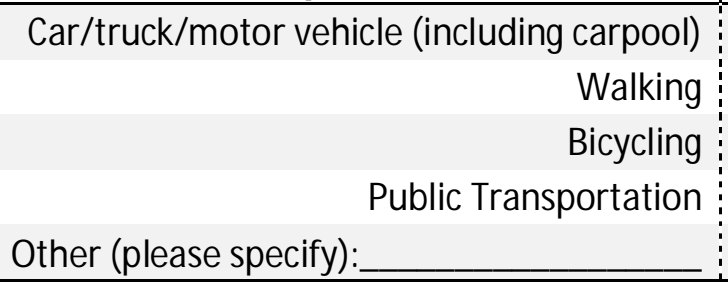

\begin{tabular}{|c:c:c|}
\hline \multicolumn{3}{|c|}{$\begin{array}{c}\text { Commute Trips } \\
\text { (to/ from work or school) }\end{array}$} \\
\hline Most Trips & Some Trips & No Trips \\
\hline$\square_{1}$ & $\square_{2}$ & $\square_{3}$ \\
$\square_{1}$ & $\square_{2}$ & $\square_{3}$ \\
$\square_{1}$ & $\square_{2}$ & $\square_{3}$ \\
$\square_{1}$ & $\square_{2}$ & $\square_{3}$ \\
$\square_{1}$ & $\square_{2}$ & $\square_{3}$ \\
\hline
\end{tabular}

Other Trips

(e.g. to the store, park, etc.)

Most Trips: Some Trips: No Trips

\section{About Your Neighborhood}

\begin{tabular}{|c|c|c|c|c|c|}
\hline $\begin{array}{l}\text { 3. Over the past two years, changes to my } \\
\text { neighborhood as a place for ... }\end{array}$ & $\begin{array}{l}\text { Very } \\
\text { Negative }\end{array}$ & $\begin{array}{l}\text { Somewhat } \\
\text { Negative }\end{array}$ & $\begin{array}{l}\text { No Impact/ } \\
\text { No Changes }\end{array}$ & $\begin{array}{l}\text { Somewhat } \\
\text { Positive }\end{array}$ & $\begin{array}{c}\text { Very } \\
\text { Positive }\end{array}$ \\
\hline walking have been & $\square_{1}$ & $\square_{2}$ & $\square_{3}$ & $\square_{4}$ & $\square_{5}$ \\
\hline driving have been . & $\square_{1}$ & $\square_{2}$ & $\square_{3}$ & $\square_{4}$ & $\square_{5}$ \\
\hline bicycling have been . & $\square_{1}$ & $\square_{2}$ & $\square_{3}$ & $\square_{4}$ & $\square_{5}$ \\
\hline taking public transportation have been . & $\mathbf{\square}_{1}$ & $\square_{2}$ & $\square_{3}$ & $\square_{4}$ & $\square_{5}$ \\
\hline
\end{tabular}

4. Overall, my level of satisfaction with transportation in my neighborhood is...

\begin{tabular}{|c:c:c:c|}
\hline $\begin{array}{c}\text { Very } \\
\text { Dissatisfied }\end{array}$ & $\begin{array}{c}\text { Somewhat } \\
\text { Dissatisfied }\end{array}$ & $\begin{array}{c}\text { Somewhat } \\
\text { Satisfied }\end{array}$ & $\begin{array}{c}\text { Very } \\
\text { Satisfied }\end{array}$ \\
\hline$\square_{1}$ & $\square_{2}$ & $\square_{3}$ & $\square_{4}$ \\
\hline
\end{tabular}

\section{About People You Encounter on the Street}

5. Please indicate if you agree or disagree with the following statements:

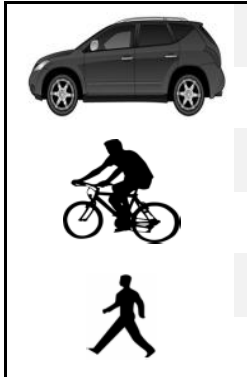

Most drivers follow the rules of the road.

M ost drivers are predictable.

M ost bicyclists follow the rules of the road.

Most bicyclists are predictable.

Most pedestrians follow the rules of the road.

Most pedestrians are predictable.

\begin{tabular}{|c|c|c|c|c|}
\hline $\begin{array}{l}\text { Strongly } \\
\text { Disagree }\end{array}$ & $\begin{array}{l}\text { Somewhat } \\
\text { Disagree }\end{array}$ & $\begin{array}{l}\text { Somewhat } \\
\text { Agree }\end{array}$ & $\begin{array}{l}\text { Strongly } \\
\text { Agree }\end{array}$ & $\begin{array}{c}\text { No } \\
\text { Opinion }\end{array}$ \\
\hline$\square_{1}$ & $\square_{2}$ & $\square_{3}$ & $\square_{4}$ & $\square_{9}$ \\
\hline$\square_{1}$ & $\square_{2}$ & $\square_{3}$ & $\square_{4}$ & $\square_{9}$ \\
\hline$\square_{1}$ & $\square_{2}$ & $\square_{3}$ & $\square_{4}$ & $\square_{9}$ \\
\hline$\square_{1}$ & $\square_{2}$ & $\square_{3}$ & $\square_{4}$ & $\square_{9}$ \\
\hline$\square_{1}$ & $\square_{2}$ & $\square_{3}$ & $\square_{4}$ & $\square_{9}$ \\
\hline$\square_{1}$ & $\square_{2}$ & $\square_{3}$ & $\square_{4}$ & $\square_{9}$ \\
\hline
\end{tabular}




\begin{tabular}{|c|c|c|c|c|c|}
\hline $\begin{array}{l}\text { 6. Please indicate whether you agree or disagree } \\
\text { with the following statements about bicycling: }\end{array}$ & $\begin{array}{l}\text { Strongly } \\
\text { Disagree }\end{array}$ & $\begin{array}{l}\text { Somewhat } \\
\text { Disagree }\end{array}$ & $\begin{array}{l}\text { Somewhat } \\
\text { Agree }\end{array}$ & $\begin{array}{c}\text { Strongly } \\
\text { Agree }\end{array}$ & $\begin{array}{c}\text { No } \\
\text { Opinion }\end{array}$ \\
\hline Bicycling in my city is a convenient way to get places. & $\mathbf{\square}_{1}$ & $\square_{2}$ & $\square_{3}$ & $\square_{4}$ & $\square_{9}$ \\
\hline Bicycling in my neighborhood is safe. & $\square_{1}$ & $\square_{2}$ & $\square_{3}$ & $\square_{4}$ & $\square_{9}$ \\
\hline $\begin{array}{r}\text { I am familiar with the bicycle lanes and other bicycle } \\
\text { facilities in my neighborhood. }\end{array}$ & $\square_{1}$ & $\square_{2}$ & $\square_{3}$ & $\square_{4}$ & $\square_{9}$ \\
\hline I would like to bicycle more often for transportation. & $\square_{1}$ & $\square_{2}$ & $\square_{3}$ & $\square_{4}$ & $\square_{9}$ \\
\hline $\begin{array}{l}\text { I would be more likely to ride a bicycle if motor vehicles } \\
\text { and bicycles were physically separated by a barrier. }\end{array}$ & $\square_{1}$ & $\square_{2}$ & $\square_{3}$ & $\square_{4}$ & $\square_{9}$ \\
\hline $\begin{array}{l}\text { Facilities that encourage bicycling for transportation are } \\
\text { a good way to improve public health. }\end{array}$ & $\square_{1}$ & $\square_{2}$ & $\square_{3}$ & $\square_{4}$ & $\square_{9}$ \\
\hline
\end{tabular}

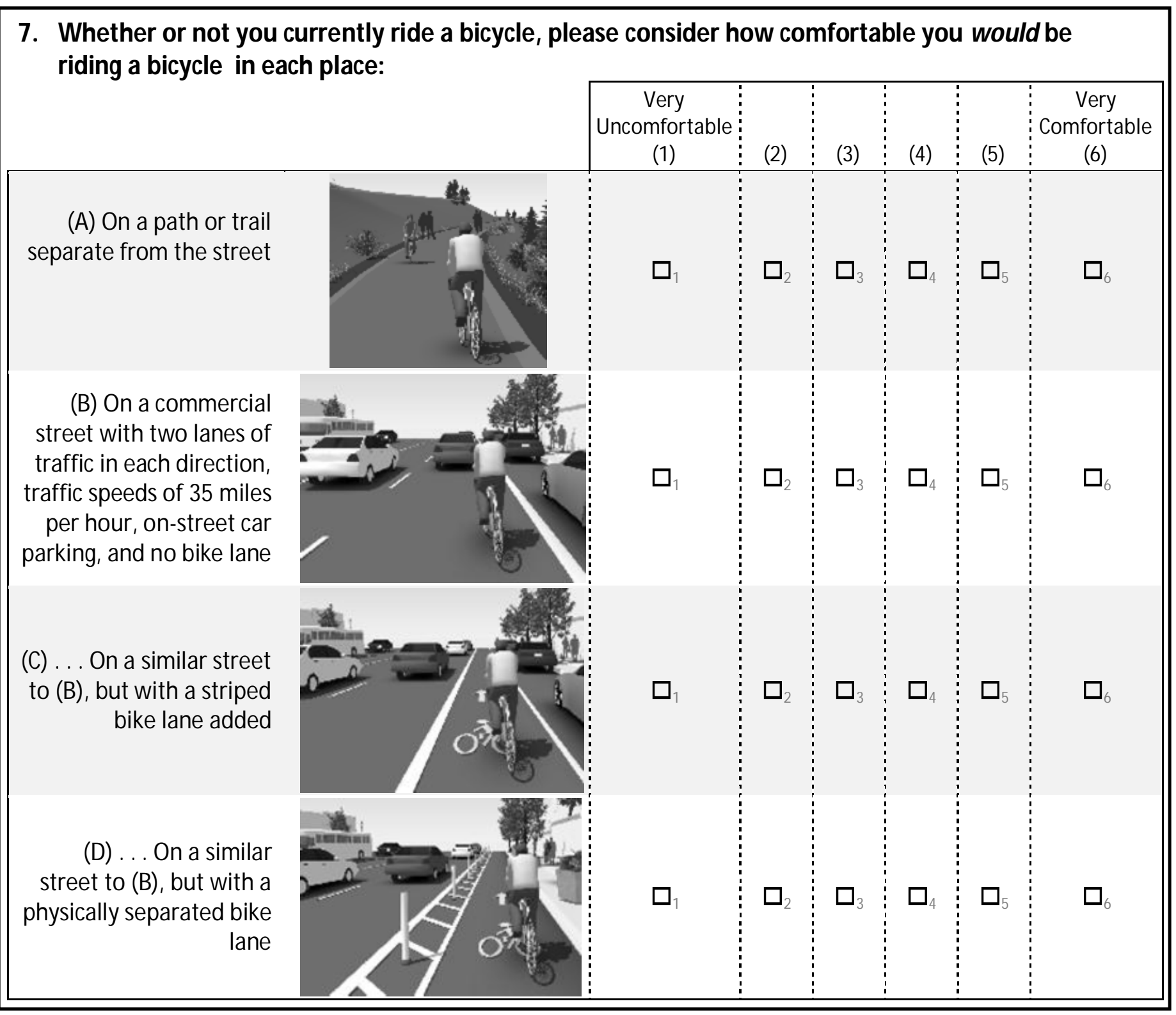




\section{North Milwaukee Avenue Protected Bike Lanes}

A protected bikeway was recently built on North M ilwaukee Avenue between North Elston Avenue and West Kinzie Street. Bike lanes are separated from other traffic lanes by a combination of a striped "buffer" zone, plastic "flexposts," or by moving parked cars away from the curb to provide a protected lane (see picture below).

North Milwaukee Avenue before and now:

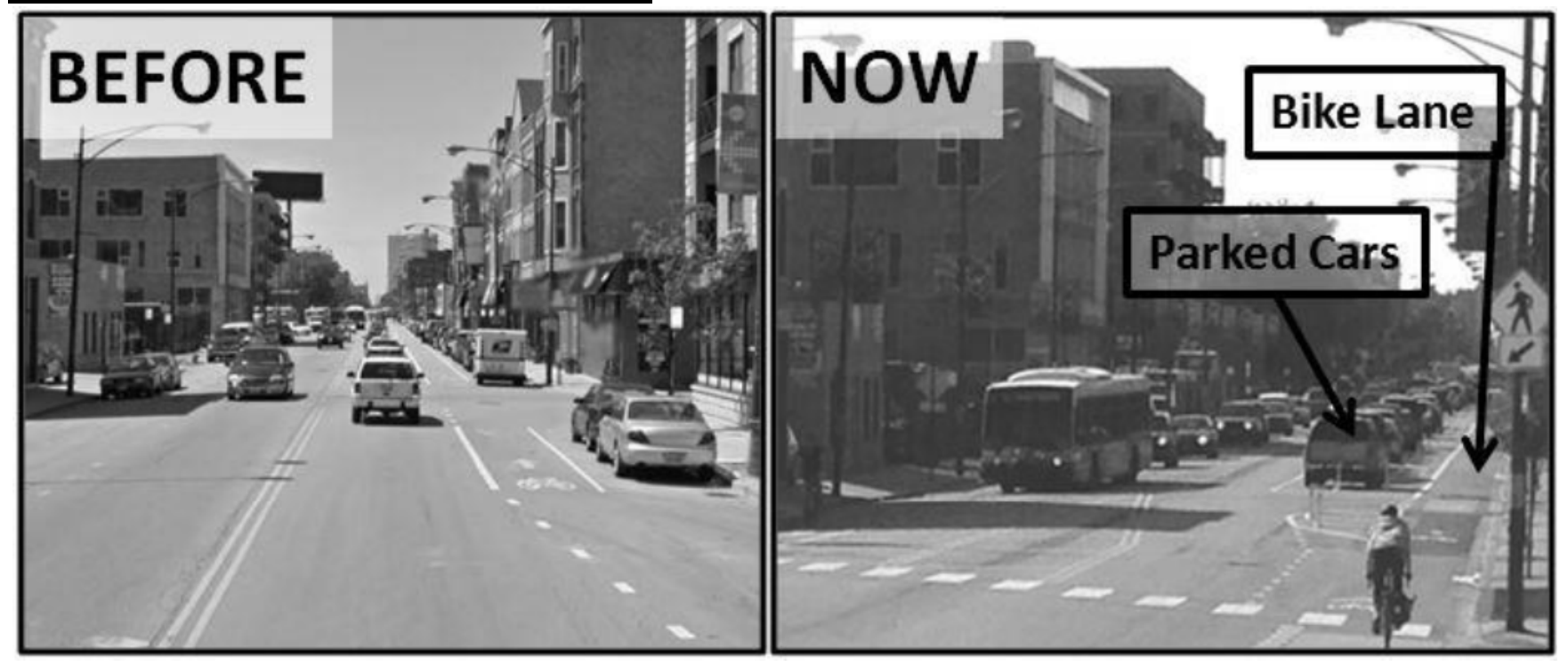

Extent of Milwaukee protected bike lanes:

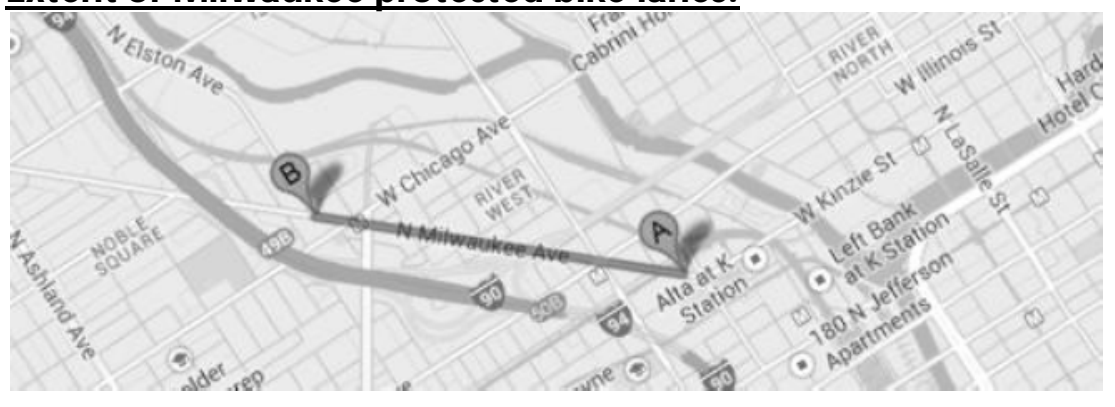

About the Milwaukee Avenue protected bike lanes

8. Because of the Milwaukee Avenue protected bike lanes ... .

\begin{tabular}{|c|c|c|c|c|c|c|}
\hline & \begin{tabular}{|c|}
$\begin{array}{c}\text { Decreased } \\
\text { a Lot }\end{array}$ \\
\end{tabular} & $\begin{array}{l}\text { Decreased } \\
\text { Somewhat }\end{array}$ & $\begin{array}{l}\text { Not } \\
\text { Changed }\end{array}$ & $\begin{array}{l}\text { Increased } \\
\text { Somewhat }\end{array}$ & $\begin{array}{c}\text { Increased } \\
\text { a Lot }\end{array}$ & \begin{tabular}{|c|} 
No \\
Opinion
\end{tabular} \\
\hline $\begin{array}{l}\text { the desirability of living in my } \\
\text { neighborhood has... }\end{array}$ & $\square_{1}$ & $\square_{2}$ & $\square_{3}$ & $\square_{4}$ & $\square_{5}$ & $\square_{9}$ \\
\hline the aesthetic appeal of M ilwaukee Avenue has ... & $\square_{1}$ & $\square_{2}$ & $\square_{3}$ & $\square_{4}$ & $\square_{5}$ & $\square_{9}$ \\
\hline the safety of DRIVING on M ilwaukee Ave has... & $\square_{1}$ & $\square_{2}$ & $\square_{3}$ & $\square_{4}$ & $\square_{5}$ & $\square_{9}$ \\
\hline the safety of BICYCLING on Milwaukee Ave has. . . & $\square_{1}$ & $\square_{2}$ & $\square_{3}$ & $\square_{4}$ & $\square_{5}$ & $\square_{9}$ \\
\hline the safety of WALKING on M ilwaukee Ave has. . & $\square_{1}$ & $\square_{2}$ & $\square_{3}$ & $\square_{4}$ & $\square_{5}$ & $\square_{9}$ \\
\hline $\begin{array}{r}\text { the number of people I see riding bikes } \\
\text { on M ilwaukee Avenue has ... }\end{array}$ & $\square_{1}$ & $\square_{2}$ & $\square_{3}$ & $\square_{4}$ & $\square_{5}$ & $\square_{9}$ \\
\hline how well the road works for all people has.. & $\square_{1}$ & $\square_{2}$ & $\square_{3}$ & $\square_{4}$ & $\square_{5}$ & $\square_{9}$ \\
\hline
\end{tabular}


About the Milwaukee Avenue protected bike lanes, cont.

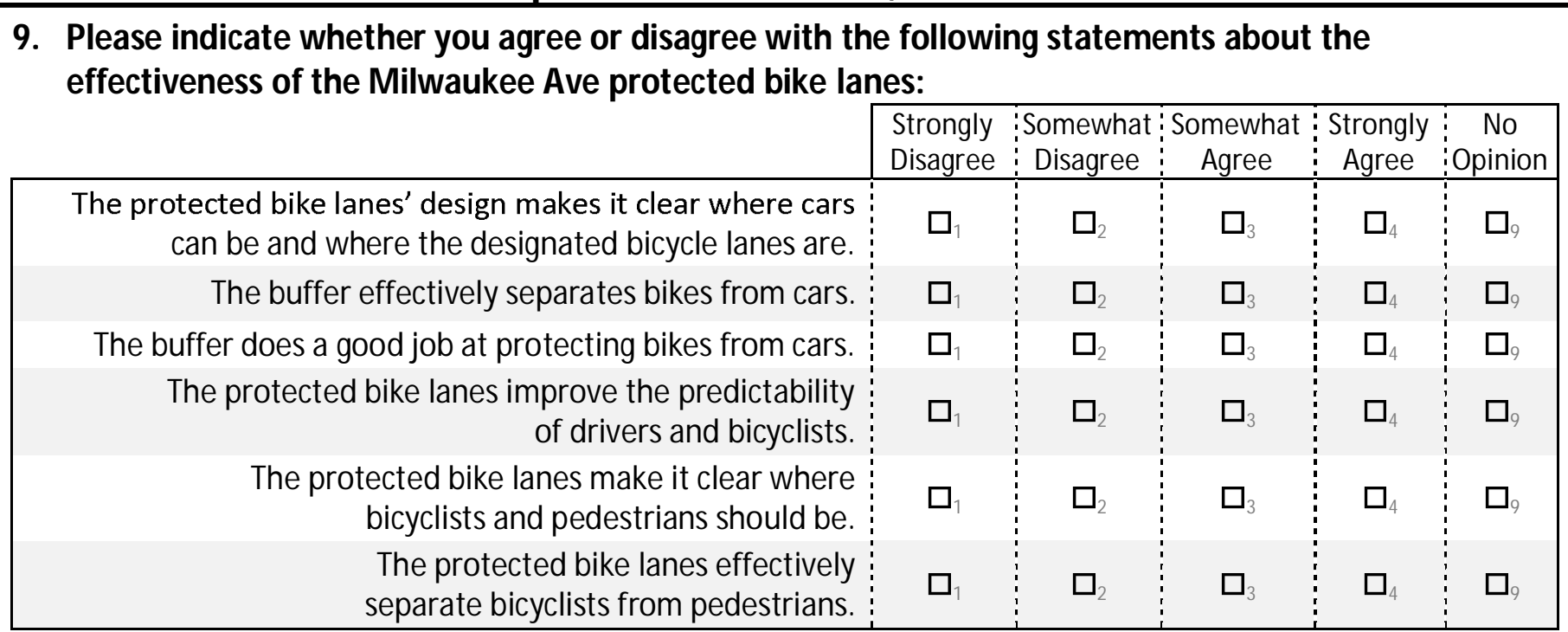

10. What do you think it means when the pavement is painted green along the

bikeway?

11. Over time, my opinion of the Milwaukee

Avenue protected bike lanes has become:

Enter response in this box

\begin{tabular}{|l|l|l:l|l}
\hline A Lot More & Somewhat & No & Somewhat & A Lot
\end{tabular} Negative More Negative Change : More Positive More Positive

12. Please indicate whether you agree or disagree with the following statements about protected bike lanes.

I would support the District in building more protected bike lanes at other locations.

Overall, I support separating bikes from cars.

\begin{tabular}{|c|c:c:c:c|}
\hline $\begin{array}{c}\text { Strongly } \\
\text { Disagree }\end{array}$ & $\begin{array}{c}\text { Somewhat } \\
\text { Disagree }\end{array}$ & $\begin{array}{c}\text { Somewhat } \\
\text { Agree }\end{array}$ & $\begin{array}{c}\text { Strongly } \\
\text { Agree }\end{array}$ & $\begin{array}{c}\text { No } \\
\text { Opinion }\end{array}$ \\
\hline$\square_{1}$ & $\square_{2}$ & $\square_{3}$ & $\square_{4}$ & $\square_{9}$ \\
$\square_{1}$ & $\square_{2}$ & $\square_{3}$ & $\square_{4}$ & $\square_{9}$ \\
\hline
\end{tabular}

\section{About Driving on Milwaukee Avenue}

13. Have you driven a motor vehicle on this section of Milwaukee Avenue since the protected bike lane were built?

$\square_{1}$ Yes (Proceed to Question 14)

$\square_{0}$ No (Skip to Question 18)

14. Since the Milwaukee Avenue protected bike lanes was built ...

the number of bicyclists riding in the same lanes with cars on M ilwaukee Avenue has . how safe and predictable bicyclists are acting has ... how safe and predictable drivers are acting has... the amount of time it takes me to drive on Milwaukee Avenue has ...

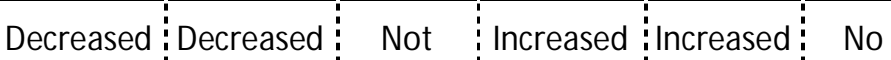
a Lot Somewhat:Changed Somewhat a Lot Opinion

\begin{tabular}{c:c:c:c:c:c} 
a Lot & Somewhat & Changed & Somewhat & a Lot & Opinion \\
$\square_{1}$ & $\square_{2}$ & $\square_{3}$ & $\square_{4}$ & $\square_{5}$ & $\square_{9}$ \\
$\square_{1}$ & $\square_{2}$ & $\square_{3}$ & $\square_{4}$ & $\square_{5}$ & $\square_{9}$ \\
$\square_{1}$ & $\square_{2}$ & $\square_{3}$ & $\square_{4}$ & $\square_{5}$ & $\square_{9}$ \\
$\square_{1}$ & $\square_{2}$ & $\square_{3}$ & $\square_{4}$ & $\square_{5}$ & $\square_{9}$ \\
\hline
\end{tabular}


About Driving on Milwaukee Avenue, cont.

\begin{tabular}{|c|c|c|c|c|c|}
\hline $\begin{array}{l}\text { 15. The impact of the Milwaukee Avenue protected } \\
\text { bike lanes on . . . }\end{array}$ & $\begin{array}{c}\text { Very } \\
\text { Negative }\end{array}$ & $\begin{array}{l}\text { Somewhat } \\
\text { Negative }\end{array}$ & $\begin{array}{c}\text { No Impact/ } \\
\text { Neutral }\end{array}$ & $\begin{array}{l}\text { Somewhat } \\
\text { Positive }\end{array}$ & $\begin{array}{l}\text { Very } \\
\text { Positive }\end{array}$ \\
\hline traffic congestion has been . . & $\square_{1}$ & $\square_{2}$ & $\square_{3}$ & $\square_{4}$ & $\square_{5}$ \\
\hline $\begin{array}{r}\text { my ability to turn off of Milwaukee Avenue at signalized } \\
\text { intersections has been ... }\end{array}$ & $\mathbf{\square}_{1}$ & $\square_{2}$ & $\square_{3}$ & $\square_{4}$ & $\square_{5}$ \\
\hline $\begin{array}{r}\text { my ability to turn off of Milwaukee Avenue into alleys, } \\
\text { driveways, and parking lots has been... }\end{array}$ & $\mathbf{\square}_{1}$ & $\square_{2}$ & $\square_{3}$ & $\square_{4}$ & $\square_{5}$ \\
\hline $\begin{array}{r}\text { my ability to pull onto Milwaukee Avenue from alleys, } \\
\text { driveways, and parking lots has been ... }\end{array}$ & $\mathbf{\square}_{1}$ & $\mathbf{\square}_{2}$ & $\square_{3}$ & $\square_{4}$ & $\square_{5}$ \\
\hline $\begin{array}{r}\text { my ability to find a parking spot on Milwaukee Avenue } \\
\text { has been ... }\end{array}$ & $\square_{1}$ & $\square_{2}$ & $\square_{3}$ & $\square_{4}$ & $\square_{5}$ \\
\hline $\begin{array}{r}\text { how stressful it is to park a car on Milwaukee Avenue } \\
\text { has been ... }\end{array}$ & $\mathbf{\square}_{1}$ & $\square_{2}$ & $\square_{3}$ & $\square_{4}$ & $\square_{5}$ \\
\hline
\end{tabular}

16. To make a right turn off of Milwaukee Avenue, motorists must cross the bike lane:

- At some intersections (PICTURE A) there is a right-turn lane to the right of the bike lane (at these locations motorists must cross the bike lane prior to the intersection).

- At other intersections (PICTURE B) motorists must cross the bike lane as they turn.

\section{Please indicate whether you agree or disagree with the following statements about turning off Milwaukee Avenue:}

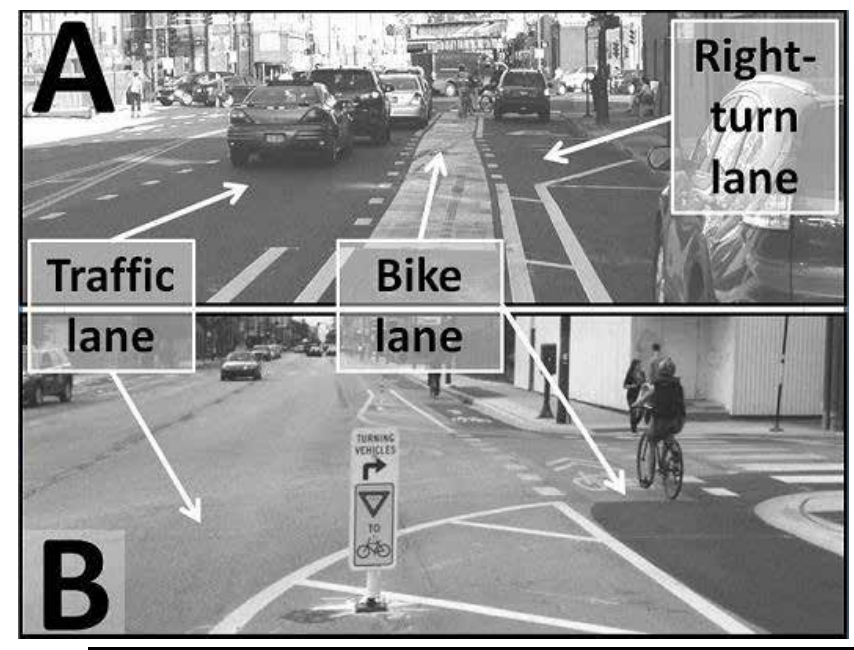

\begin{tabular}{|l|l|l|}
\hline Strongly & Somewhat Somewhat: Strongly & No \\
\hline
\end{tabular} Disagree Disagree Agree Agree OOpinion

\begin{tabular}{|c|c|c|c|c|c|c|}
\hline $\begin{array}{l}\infty \\
\infty \\
\ll\end{array}$ & $\begin{array}{r}\text { When I want to make a right turn off of Milwaukee } \\
\text { Avenue, it is generally clear where I should cross over } \\
\text { the bike lane. }\end{array}$ & $\square_{1}$ & $\square_{2}$ & $\square_{3}$ & $\square_{4}$ & $\square_{9}$ \\
\hline \multirow[t]{2}{*}{$\varangle$} & $\begin{array}{r}\text { At intersections like Picture } \mathbf{A} \text { above: When I want to } \\
\text { turn right, I am able to adequately see if there are any } \\
\text { approaching cyclists in the bike lane. }\end{array}$ & $\square_{1}$ & $\square_{2}$ & $\square_{3}$ & $\square_{4}$ & $\square_{9}$ \\
\hline & $\begin{array}{l}\text { I prefer to merge across the bike lane prior to arriving } \\
\text { at an intersection (like in Picture A). }\end{array}$ & $\square_{1}$ & $\mathbf{D}_{2}$ & $\square_{3}$ & $\square_{4}$ & $\square_{9}$ \\
\hline \multirow{2}{*}{$\infty$} & $\begin{array}{r}\text { At intersections like Picture B above: When I want to } \\
\text { turn right, I am able to adequately see if there are any } \\
\text { approaching cyclists in the bike lane. }\end{array}$ & $\square_{1}$ & $\square_{2}$ & $\square_{3}$ & $\square_{4}$ & $\square_{9}$ \\
\hline & $\begin{array}{l}\text { The "Yield to Bikes" signs (like in Picture B) have made } \\
\text { me pay closer attention to cyclists when turning off } \\
\text { M ilwaukee Ave. }\end{array}$ & $\square_{1}$ & $\square_{2}$ & $\square_{3}$ & $\square_{4}$ & $\square_{9}$ \\
\hline
\end{tabular}

17. Do you avoid driving on Milwaukee Avenue because of the protected bike lanes?

Yes No 
18. Have you bicycled on this section of Milwaukee Avenue since the protected lanes were built?

$\square_{1}$ Yes (Proceed to Question 19) $\square_{0}$ No (Skip to Question 23)

19. How comfortable do you feel when bicycling in the Milwaukee Avenue protected bike lanes?

\begin{tabular}{|c|c|c|c|c|c|}
\hline $\begin{array}{c}\text { Very } \\
\text { Uncomfortable } \\
(1)\end{array}$ & (2) & (3) & (4) & (5) & $\begin{array}{c}\text { Very } \\
\text { Comfortable } \\
(6)\end{array}$ \\
\hline$\square_{1}$ & $\square_{2}$ & $\square_{3}$ & $\square_{4}$ & $\square_{5}$ & $\square_{6}$ \\
\hline
\end{tabular}

20. Because of the Milwaukee Avenue protected bike lanes...

\begin{tabular}{|c|c|c|c|c|c|c|}
\hline & $\begin{array}{c}\text { Decreased } \\
\text { a Lot }\end{array}$ & $\begin{array}{l}\text { Decreased } \\
\text { Somewhat }\end{array}$ & $\begin{array}{c}\text { Not } \\
\text { Changed }\end{array}$ & $\begin{array}{l}\text { Increased } \\
\text { Somewhat }\end{array}$ & $\begin{array}{c}\text { Increased } \\
\text { a Lot }\end{array}$ & $\begin{array}{l}\text { No } \\
\text { Opinion }\end{array}$ \\
\hline $\begin{array}{r}\text { the likelihood that I will choose to bicycle on } \\
\text { M ilwaukee Avenue as opposed to other streets } \\
\text { has... }\end{array}$ & $\square_{1}$ & $\square_{2}$ & $\square_{3}$ & $\square_{4}$ & $\square_{5}$ & $\square_{9}$ \\
\hline $\begin{array}{r}\text { the time it takes me to bicycle on Milwaukee } \\
\text { Avenue has... }\end{array}$ & $\square_{1}$ & $\square_{2}$ & $\square_{3}$ & $\square_{4}$ & $\square_{5}$ & $\square_{9}$ \\
\hline $\begin{array}{l}\text { the usefulness of M ilwaukee Avenue for getting } \\
\text { places I want to go has... }\end{array}$ & $\square_{1}$ & $\square_{2}$ & $\square_{3}$ & $\square_{4}$ & $\square_{5}$ & $\square_{9}$ \\
\hline $\begin{array}{r}\text { how often I stop at shops and businesses } \\
\text { on M ilwaukee Avenue has... }\end{array}$ & $\square_{1}$ & $\square_{2}$ & $\square_{3}$ & $\square_{4}$ & $\square_{5}$ & $\square_{9}$ \\
\hline $\begin{array}{r}\text { drivers' awareness of bicyclists on M ilwaukee } \\
\text { Avenue has... }\end{array}$ & $\square_{1}$ & $\square_{2}$ & $\square_{3}$ & $\square_{4}$ & $\square_{5}$ & $\square_{9}$ \\
\hline drivers' speeds on M ilwaukee Avenue have ... & $\square_{1}$ & $\square_{2}$ & $\square_{3}$ & $\square_{4}$ & $\square_{5}$ & $\square_{9}$ \\
\hline $\begin{array}{r}\text { how comfortable I feel when bicycling } \\
\text { on M ilwaukee Avenue has... }\end{array}$ & $\square_{1}$ & $\square_{2}$ & $\square_{3}$ & $\square_{4}$ & $\square_{5}$ & $\square_{9}$ \\
\hline how often I ride a bicycle overall has... & $\square_{1}$ & $\square_{2}$ & $\square_{3}$ & $\square_{4}$ & $\square_{5}$ & $\square_{9}$ \\
\hline $\begin{array}{l}\text { the difficulty of making a right-turn off of } \\
\text { Milwaukee Avenue while bicycling has... }\end{array}$ & $\square_{1}$ & $\square_{2}$ & $\square_{3}$ & $\square_{4}$ & $\square_{5}$ & $\square_{9}$ \\
\hline $\begin{array}{l}\text { the difficulty of navigating around } \\
\text { turning motor vehicles has... }\end{array}$ & $\square_{1}$ & $\square_{2}$ & $\square_{3}$ & $\square_{4}$ & $\square_{5}$ & $\square_{9}$ \\
\hline $\begin{array}{r}\text { the difficulty of navigating around } \\
\text { pedestrians has... }\end{array}$ & $\square_{1}$ & $\square_{2}$ & $\square_{3}$ & $\square_{4}$ & $\square_{5}$ & $\square_{9}$ \\
\hline
\end{tabular}

21. In the past 30 days, how many days have you bicycled for transportation (i.e. not just for recreation/ exercise)?

22. Did you fill out a separate online bicyclist survey about the Milwaukee Avenue protected bike lanes from us recently?
$\square$ Yes
No 
23. Have you walked on this section of Milwaukee Avenue since the protected bike lanes were built?
Yes (Proceed to Question 24)
No (Skip to Question 27)

24. How often do bicyclists in the bike lanes stop for pedestrians at unsignalized intersections?

\begin{tabular}{|c:c:c:c|}
\hline Usually & Sometimes & Rarely & Never \\
\hline$\square_{1}$ & $\square_{2}$ & $\square_{3}$ & $\square_{4}$ \\
\hline
\end{tabular}

25. Because of the Milwaukee Avenue \begin{tabular}{ll|l|l|l|l} 
Decreased & Decreased & Not & Increased & Increased & No
\end{tabular} protected bike lanes... .

drivers' speeds on Milw. Ave. have generally ... the number of bicyclists riding on the sidewalk has... my satisfaction with the walking environment on Milwaukee Avenue has ... my sense of safety when crossing M ilwaukee Avenue has... a Lot Somewhat Changed Somewhat a Lot Opinion

26. Do you ever walk or jog in the protected bike lanes, rather than on the sidewalk?

$\square_{0}$ No

$\square_{1}$ Yes (Please explain why you choose the bikelane over the sidewalk in the space to the right) $\rightarrow$

\section{About Visiting Businesses on Milwaukee Avenue}

27. How many days per month do you spend money at businesses on or near Milwaukee Avenue?

(Enter 0 if None) days

28. How did you travel to get there?

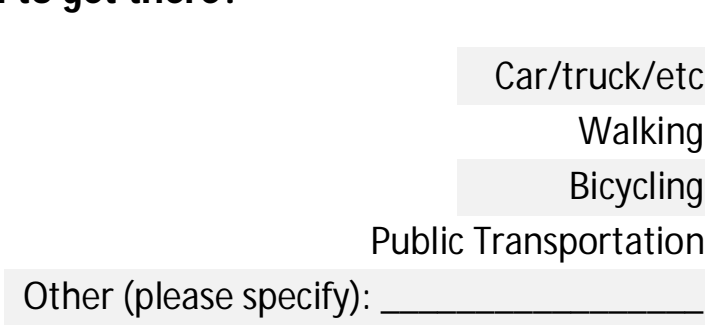

\begin{tabular}{|c:c:c|}
\hline $\begin{array}{c}\text { Most of the } \\
\text { time }\end{array}$ & $\begin{array}{c}\text { Some of the } \\
\text { time }\end{array}$ & Never \\
\hline$\square_{1}$ & $\square_{2}$ & $\square_{3}$ \\
$\square_{1}$ & $\square_{2}$ & $\square_{3}$ \\
$\square_{1}$ & $\square_{2}$ & $\square_{3}$ \\
$\square_{1}$ & $\square_{2}$ & $\square_{3}$ \\
$\square_{1}$ & $\square_{2}$ & $\square_{3}$ \\
\hline
\end{tabular}

29. What types of businesses in the area do you patronize? (check all that apply)
$\square_{1}$ Retail Shops
$\square_{2}$ Banks
$\square_{3}$ Restaurants
$\square_{4}$ Bars
Coffee Shop
$\square_{6}$ Other ->

30. When you visit this area, how many businesses do you typically go to each time? (select the best response)
$\square_{1} 1$
$\square_{2} 2$
$\square_{3} 3$ or more

31. Since the protected bike lanes were built, are you more or less likely to visit a business on Milwaukee Avenue?

$\square_{1}$ A lot less likely
$\square_{2}$ Somewhat less likely
$\square_{3}$ No change
$\square_{4}$ Somewhat more likely
$\square_{5}$ A lot more likely


32. What is the best thing about the protected bike lanes?
33. What is the worst thing about the protected bike lanes?

\section{About You}

We have a few questions about you so that we may understand the characteristics of our survey respondents. We will keep this information confidential and it is not linked to your name
34. Do you have a current:
Driver's license
Transit pass
Bikeshare (Divvy) Membership
Carshare M embership

35. How many working motor vehicles does your household own or lease?

(Do not include motorhomes)

$$
\begin{aligned}
& \text { \#Vehicles } \\
& \text { (Enter } 0 \text { if None) }
\end{aligned}
$$

36. How many working adult bicycles does your household own?

$$
\begin{aligned}
& \text { \#Bicycles } \\
& \text { (Enter } 0 \text { if } \\
& \text { None) }
\end{aligned}
$$

37. INCLUDING YOURSELF, how many people live in your household?

$$
\begin{aligned}
& \text { \#Adults } \\
& \text { \#Children } \\
& \begin{array}{l}
\text { (Enter } 0 \text { if } \\
\text { if } \\
\text { None })
\end{array}
\end{aligned}
$$

38. How long have you lived at this home?

$$
\text { \#Years }
$$

39. Do you rent or own your home?

$$
\square_{1} \text { Rent } \quad \square_{2} \text { Own } \quad \square_{3} \text { Other }
$$

40. Do you consider yourself:

(Select all that apply)

$\square$ American Indian or Alaska Native
$\square$ Asian
$\square$ Black or African American
$\square$ Hispanic or Latino/a
$\square$ White or Caucasian
$\square$ Other:

$\square$ American Indian or Alaska Native

$\square$ Asian

$\square$ Black or African American

$\square$ Other:

Thank you for taking our survey! If you would like to be entered in a random drawing for one of three $\$ 100$ Amazon gift cards please enter your name and contact information on the raffle slip you received with the survey.

41. What is your age? years

42. What is your gender?

43. What is your employment status?

(Select all that apply)

$\square$ I work outside the home-> What is the zip code of your place of work?

$\square$ I go to school outside the home->What is the zip code of your school?

$\square$ I work from home

$\square$ Not employed at this time

44. What is your annual household income?
$\square_{1}$ Less than $\$ 25,000$
$\square, \$ 25,000$ to less than $\$ 50,000$
$\square_{3} \$ 50,000$ to less than $\$ 75,000$
$\square_{4} \$ 75,000$ to less than $\$ 100,000$
$\square_{5} \$ 100,000$ to less than $\$ 200,000$
$\square_{6} \$ 200,000$ or more
$\square_{9}$ I prefer not to provide this information

45. What is the highest level of school you have completed?
$\square_{1}$ Some high school or less
$\square_{2}$ High school diploma or GED
$\square_{3}$ Some College
$\square$ Trade/Vocational School
$\square_{5}$ Associate Degree
$\square$ Four-year college degree or more
$\square$ Other:

46. Is there anything else you'd like to tell us? (Use the space below) 


\section{RESIDENT: CHICAGo - DEARBoRn AVE.}




\section{Neighborhood Street Study}

About How You Get Around
(1) Complete this paper survey and the enclosed raffle slip. Return in the postage-paid envelope;

OR

(2) Complete the online survey and raffle entry at: http:// tinyurl.com/ DearbornStudy To take the online survey, you will need to enter this code: D00013

Survey deadline 10/ 17/ 2013

\begin{tabular}{|c|c|c|c|c|c|c|c|c|}
\hline \multirow{2}{*}{\multicolumn{2}{|c|}{$\begin{array}{l}\text { 1. For each mode of transportation, } \\
\text { please indicate your level of use: }\end{array}$}} & \multicolumn{3}{|c|}{$\begin{array}{c}\text { Commute Trips } \\
\text { (to/from work or school) }\end{array}$} & \multicolumn{4}{|c|}{$\begin{array}{c}\text { Other Trips } \\
\text { (e.g. to the store, park, etc.) }\end{array}$} \\
\hline & & Most Trips & Some Trips & No Trips & Most Trips: & Som & e Trips & No Trips \\
\hline \multicolumn{2}{|r|}{ Car/truck/motor vehicle (including carpool) } & $\square_{1}$ & $\square_{2}$ & $\square_{3}$ & $\square_{1}$ & \multicolumn{2}{|c|}{$\square_{2}$} & $\square_{3}$ \\
\hline & Walking & $\square_{1}$ & $\square_{2}$ & $\square_{3}$ & $\square_{1}$ & \multicolumn{2}{|c|}{$\square_{2}$} & $\square_{3}$ \\
\hline & Bicycling & $\square_{1}$ & $\square_{2}$ & $\square_{3}$ & $\square_{1}$ & \multicolumn{2}{|r|}{$\square_{2}$} & $\square_{3}$ \\
\hline & Public Transportation & $\square_{1}$ & $\square_{2}$ & $\square_{3}$ & $\square_{1}$ & \multicolumn{2}{|c|}{$\square_{2}$} & $\square_{3}$ \\
\hline \multicolumn{2}{|r|}{ Other (please specify): } & $\square_{1}$ & $\square_{2}$ & $\square_{3}$ & $\square_{1}$ & \multicolumn{2}{|c|}{$\square_{2}$} & $\square_{3}$ \\
\hline \multicolumn{4}{|c|}{$\begin{array}{l}\text { 2. Compared to two years ago, are you taking more or } \\
\text { fewer trips by each mode of transportation? }\end{array}$} & M ore Trips & \multicolumn{2}{|c|}{ No Change } & \multicolumn{2}{|c|}{ Fewer Trips } \\
\hline \multicolumn{4}{|c|}{ Car/truck/motor vehicle (including carpool) } & $\square_{1}$ & \multicolumn{2}{|c|}{$\square_{2}$} & \multicolumn{2}{|c|}{$\square_{3}$} \\
\hline \multicolumn{4}{|c|}{ Walking } & $\square_{1}$ & \multicolumn{2}{|l|}{$\square_{2}$} & \multicolumn{2}{|r|}{$\square_{3}$} \\
\hline \multicolumn{4}{|r|}{ Bicycling } & $\square_{1}$ & \multicolumn{2}{|l|}{$\square_{2}$} & \multicolumn{2}{|r|}{$\square_{3}$} \\
\hline \multicolumn{4}{|c|}{ Public Transportation } & $\square_{1}$ & \multicolumn{2}{|l|}{$\square_{2}$} & \multicolumn{2}{|r|}{$\square_{3}$} \\
\hline \multicolumn{4}{|c|}{ Other (please specify): } & $\square_{1}$ & \multicolumn{2}{|l|}{$\square_{2}$} & \multicolumn{2}{|r|}{$\square_{3}$} \\
\hline
\end{tabular}

About Your Neighborhood

3. Over the past two years, changes to my neighborhood as a place for ...

Very Somewhat No Impact/ Somewhat Very Negative Negative $:$ No Changes: Positive $:$ Positive

walking have been . .
driving have been ...
bicycling have been ...
taking public transportation have been ...

$\begin{array}{l:l}\square_{1} & \square_{2}\end{array}$

$a_{2}$




\begin{tabular}{|c|c|c|c|c|c|}
\hline $\begin{array}{l}\text { 6. Please indicate whether you agree or disagree } \\
\text { with the following statements about bicycling: }\end{array}$ & $\begin{array}{l}\text { Strongly } \\
\text { Disagree }\end{array}$ & $\begin{array}{l}\text { Somewhat } \\
\text { Disagree }\end{array}$ & $\begin{array}{l}\text { Somewhat } \\
\text { Agree }\end{array}$ & $\begin{array}{c}\text { Strongly } \\
\text { Agree }\end{array}$ & $\begin{array}{c}\text { No } \\
\text { Opinion }\end{array}$ \\
\hline Bicycling in my city is a convenient way to get places. & $\mathbf{\square}_{1}$ & $\square_{2}$ & $\square_{3}$ & $\square_{4}$ & $\square_{9}$ \\
\hline Bicycling in my neighborhood is safe. & $\square_{1}$ & $\square_{2}$ & $\square_{3}$ & $\square_{4}$ & $\square_{9}$ \\
\hline $\begin{array}{r}\text { I am familiar with the bicycle lanes and other bicycle } \\
\text { facilities in my neighborhood. }\end{array}$ & $\square_{1}$ & $\square_{2}$ & $\square_{3}$ & $\square_{4}$ & $\square_{9}$ \\
\hline I would like to bicycle more often for transportation. & $\square_{1}$ & $\square_{2}$ & $\square_{3}$ & $\square_{4}$ & $\square_{9}$ \\
\hline $\begin{array}{l}\text { I would be more likely to ride a bicycle if motor vehicles } \\
\text { and bicycles were physically separated by a barrier. }\end{array}$ & $\square_{1}$ & $\square_{2}$ & $\square_{3}$ & $\square_{4}$ & $\square_{9}$ \\
\hline $\begin{array}{l}\text { Facilities that encourage bicycling for transportation are } \\
\text { a good way to improve public health. }\end{array}$ & $\square_{1}$ & $\square_{2}$ & $\square_{3}$ & $\square_{4}$ & $\square_{9}$ \\
\hline
\end{tabular}

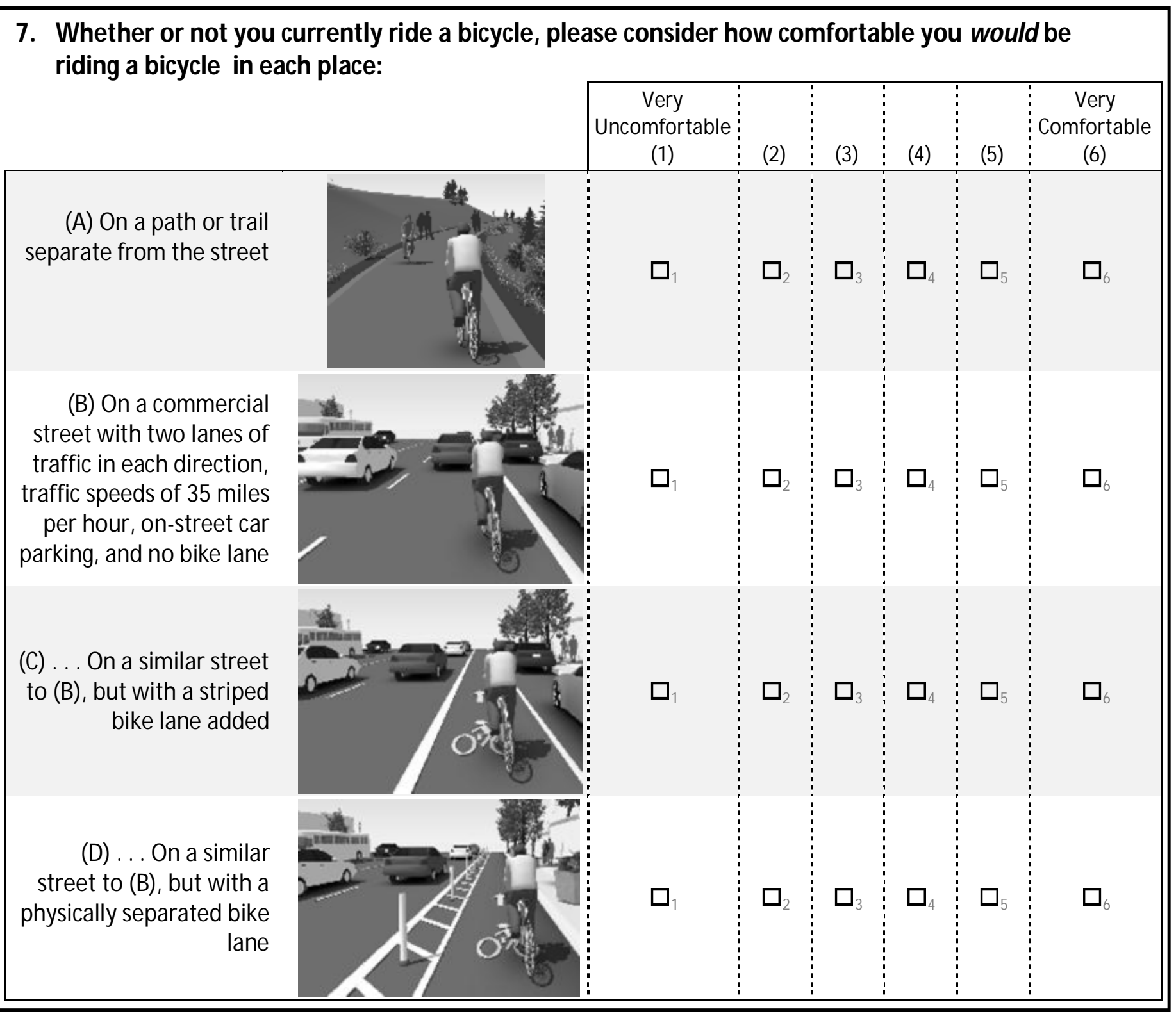




\section{Dearborn Street Protected Bike Lanes}

A two-way protected bikeway was recently built on Dearborn Street between West Kinzie Street and West Polk Street. Bike lanes are separated from other traffic lanes by a combination of a striped "buffer" zone, plastic "flexposts" and by moving parked cars away from the curb to provide a protected lane (see picture below). Bicycle signals and left-turn signals were added at many intersections as well.

Dearborn Street:
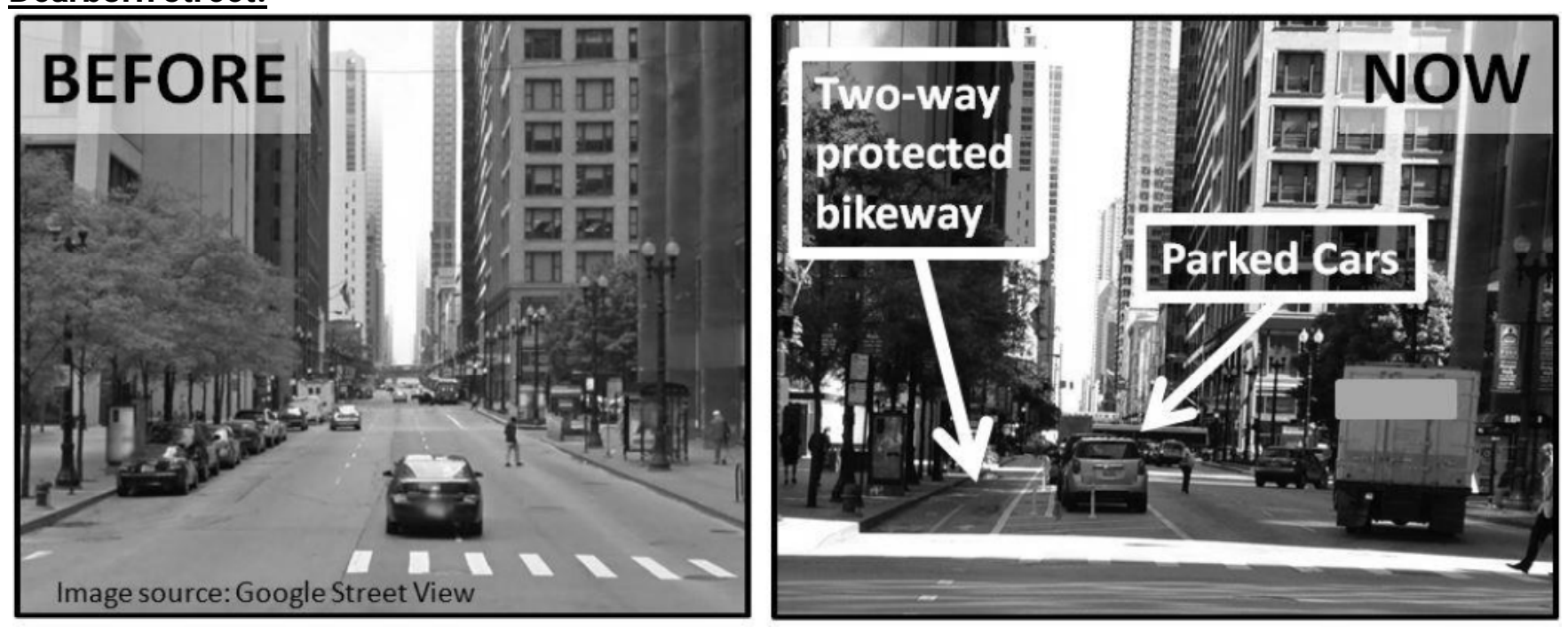

Extent of Dearborn Street protected bike lanes:

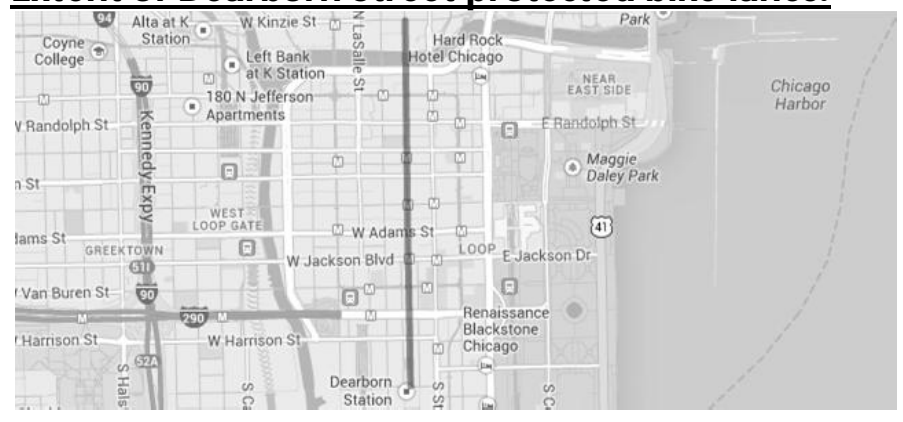

\section{About the Dearborn Street protected bike lanes}

8. Because of the Dearborn Street protected bike lanes....

\begin{tabular}{|c|c|c|c|c|c|c|}
\hline & $\begin{array}{c}\text { Decreased } \\
\text { a Lot }\end{array}$ & $\begin{array}{l}\text { Decreased } \\
\text { Somewhat }\end{array}$ & $\begin{array}{c}\text { Not } \\
\text { Changed }\end{array}$ & $\begin{array}{l}\text { Increased } \\
\text { Somewhat }\end{array}$ & $\begin{array}{c}\text { Increased } \\
\text { a Lot }\end{array}$ & \begin{tabular}{|l} 
No \\
Opinion
\end{tabular} \\
\hline $\begin{array}{r}\text { the desirability of living in my } \\
\text { neighborhood has... }\end{array}$ & $\square_{1}$ & $\square_{2}$ & $\square_{3}$ & $\square_{4}$ & $\square_{5}$ & $\square_{9}$ \\
\hline the aesthetic appeal of Dearborn Street has ... & $\square_{1}$ & $\square_{2}$ & $\square_{3}$ & $\square_{4}$ & $\square_{5}$ & $\square_{9}$ \\
\hline the safety of DRIVING on Dearborn Street has ... & $\square_{1}$ & $\square_{2}$ & $\square_{3}$ & $\square_{4}$ & $\square_{5}$ & $\square_{9}$ \\
\hline the safety of BICYCLING on Dearborn St. has. . . & $\square_{1}$ & $\square_{2}$ & $\square_{3}$ & $\square_{4}$ & $\square_{5}$ & $\square_{9}$ \\
\hline the safety of WALKING on Dearborn St. has . . . & $\square_{1}$ & $\square_{2}$ & $\square_{3}$ & $\square_{4}$ & $\square_{5}$ & $\square_{9}$ \\
\hline $\begin{array}{r}\text { the number of people I see riding bikes } \\
\text { on Dearborn Street has... }\end{array}$ & $\square_{1}$ & $\square_{2}$ & $\square_{3}$ & $\square_{4}$ & $\square_{5}$ & $\square_{9}$ \\
\hline how well the road works for all people has. & $\square_{1}$ & $\square_{2}$ & $\square_{3}$ & $\square_{4}$ & $\square_{5}$ & $\square_{9}$ \\
\hline
\end{tabular}


About the Dearborn Street protected bike lanes, cont.

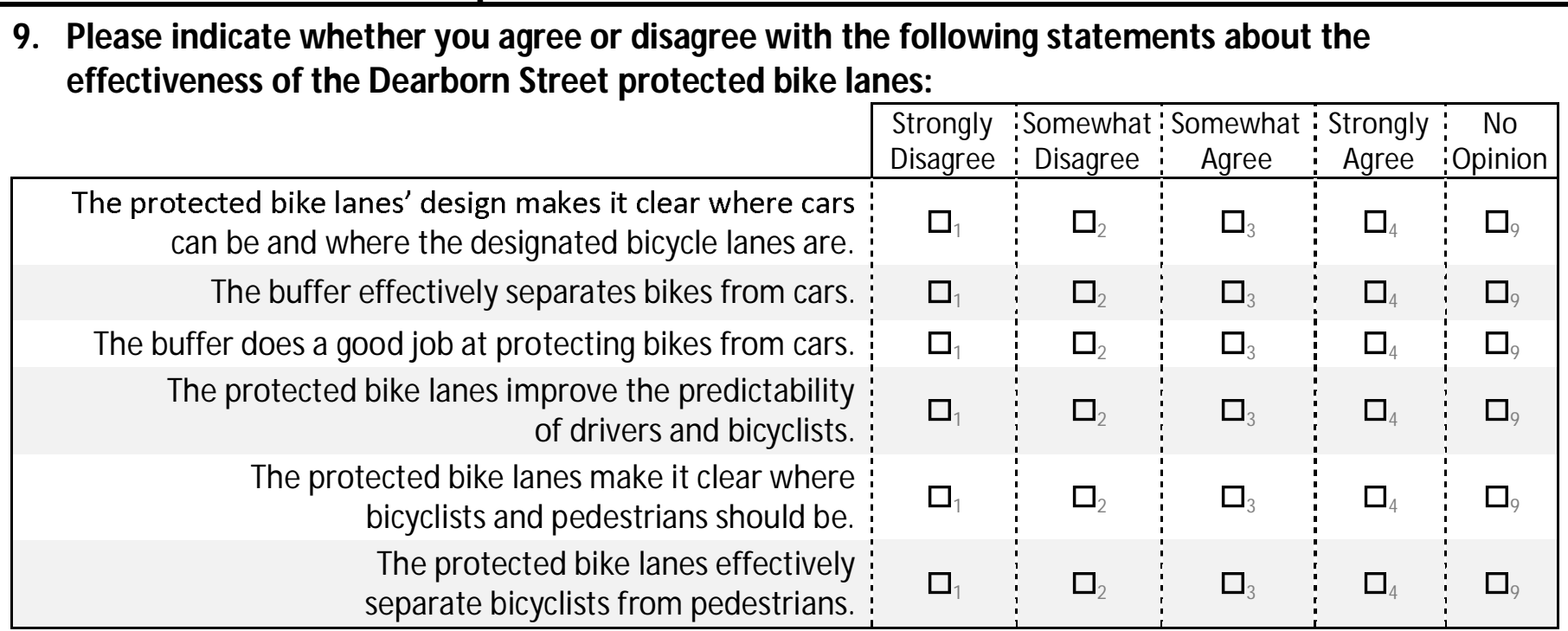

10. What do you think it means when the pavement is painted green along the

bikeway?

11. Over time, my opinion of the Dearborn

Street protected bike lanes has become:

Enter response in this box

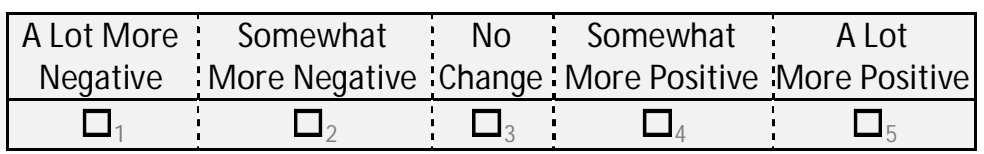

12. Please indicate whether you agree or disagree with the following statements about protected bike lanes.

I would support the District in building more protected bike lanes at other locations.

Overall, I support separating bikes from cars.

\begin{tabular}{|c|c:c:c:c|}
\hline $\begin{array}{c}\text { Strongly } \\
\text { Disagree }\end{array}$ & $\begin{array}{c}\text { Somewhat } \\
\text { Disagree }\end{array}$ & $\begin{array}{c}\text { Somewhat } \\
\text { Agree }\end{array}$ & $\begin{array}{c}\text { Strongly } \\
\text { Agree }\end{array}$ & $\begin{array}{c}\text { No } \\
\text { Opinion }\end{array}$ \\
\hline$\square_{1}$ & $\square_{2}$ & $\square_{3}$ & $\square_{4}$ & $\square_{9}$ \\
$\square_{1}$ & $\square_{2}$ & $\square_{3}$ & $\square_{4}$ & $\square_{9}$ \\
\hline
\end{tabular}

\section{About Driving on Dearborn Street}

13. Have you driven a motor vehicle on this section of Dearborn Street since the protected bike lanes were built?

$\square_{1}$ Yes (Proceed to Question 14)

$\square_{0}$ No (Skip to Question 20)

14. Since the Dearborn Street protected bike lanes was built ...

the number of bicyclists riding in the same lanes with cars on Dearborn Street has .

how safe and predictable bicyclists are acting has...

how safe and predictable drivers are acting has .. the amount of time it takes me to drive on Dearborn Street has...

Decreased Decreased $\quad$ Not Increased Increased a Lot Somewhat:Changed Somewhat a Lot Opinion

$\begin{array}{l:l:l}\square_{1} & \square_{2} & \square_{3} \\ \square_{1} & \square_{2} & \square_{3} \\ \square_{1} & \square_{2} & \square_{3} \\ \square_{1} & \square_{2} & \square_{3}\end{array}$

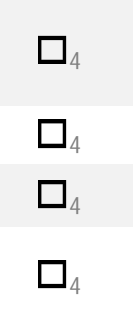


About Driving on Dearborn Street, cont.

\section{The impact of the Dearborn Street protected} bike lanes on ....

\begin{tabular}{|c|c|c|c|c|c|}
\hline traffic congestion has been ... & $\square_{1}$ & $\square_{2}$ & $\square_{3}$ & $\square_{4}$ & $\square_{5}$ \\
\hline $\begin{array}{r}\text { my ability to turn off of Dearborn Street at signalized } \\
\text { intersections has been . . }\end{array}$ & $\square_{1}$ & $\square_{2}$ & $\square_{3}$ & $\square_{4}$ & $\square_{5}$ \\
\hline $\begin{array}{r}\text { my ability to turn off of Dearborn Street into alleys, } \\
\text { driveways, and parking garages has been ... }\end{array}$ & $\square_{1}$ & $\square_{2}$ & $\square_{3}$ & $\square_{4}$ & $\square_{5}$ \\
\hline $\begin{array}{l}\text { my ability to pull onto Dearborn Street from alleys, } \\
\text { driveways, and parking garages has been ... }\end{array}$ & $\square_{1}$ & $\square_{2}$ & $\square_{3}$ & $\square_{4}$ & $\square_{5}$ \\
\hline $\begin{array}{r}\text { my ability to find a parking spot on Dearborn Street has } \\
\text { been } . .\end{array}$ & $\square_{1}$ & $\square_{2}$ & $\square_{3}$ & $\square_{4}$ & $\square_{5}$ \\
\hline $\begin{array}{r}\text { how stressful it is to park a car on Dearborn Street has } \\
\text { been } . . .\end{array}$ & $\square_{1}$ & $\square_{2}$ & $\square_{3}$ & $\square_{4}$ & $\square_{5}$ \\
\hline
\end{tabular}

\section{At many intersections along Dearborn} Street, new bicycle signals were added, along with a new left-turn signal for motor vehicles in the left turn lane (see picture).

Prior to taking this survey, had you noticed the bicycle signals on Dearborn Street?

$\square_{0}$ No $\square_{1}$ Yes

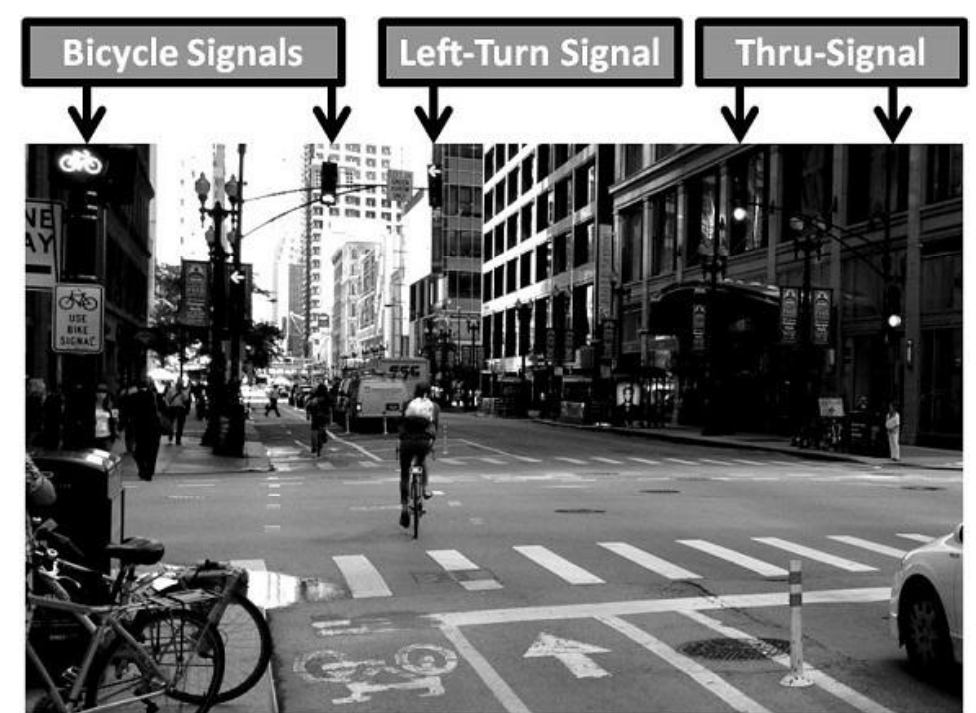

17. Please indicate whether you agree or disagree with the following statements.

At these intersections, it is always clear to me which signal I should use as a motorist. Using the small bicycle in the bicycle signal lens is a good way to communicate the signal is only for bicycles.

\begin{tabular}{|c|c|c|c|c|}
\hline $\begin{array}{l}\text { Strongly } \\
\text { Disagree }\end{array}$ & $\begin{array}{l}\text { Somewhat } \\
\text { Disagre }\end{array}$ & $\begin{array}{l}\text { Somewhat } \\
\text { Agree }\end{array}$ & $\begin{array}{c}\text { Strongly } \\
\text { Agree }\end{array}$ & $\begin{array}{c}\text { No } \\
\text { Opinion }\end{array}$ \\
\hline $\mathbf{\square}_{1}$ & $\square_{2}$ & $\square_{3}$ & $\square_{4}$ & $\square_{9}$ \\
\hline$\square_{1}$ & $\square_{2}$ & $\square_{3}$ & $\square_{4}$ & $\square_{9}$ \\
\hline
\end{tabular}

18. When making a left-turn off of Dearborn Street, how often ....

... do you encounter bicyclists in your path when attempting to make a left turn?

. do you encounter pedestrians in your path when attempting to make a left turn?

\begin{tabular}{|c|c:c:c:c|}
\hline Never & Rarely & Sometimes & Often & N/A \\
\hline$\square_{1}$ & $\square_{2}$ & $\square_{3}$ & $\square_{4}$ & $\square_{9}$ \\
$\square_{1}$ & $\square_{2}$ & $\square_{3}$ & $\square_{4}$ & $\square_{9}$ \\
\hline
\end{tabular}

19. Do you avoid driving on Dearborn Street because of the protected bike lanes?
$\square_{1}$ Yes
$\square_{0}$ No 
About Bicycling in the Dearborn Street protected bike lanes

20. Have you bicycled on this section of Dearborn Street since the protected lanes were built?

$\square_{1}$ Yes (Proceed to Question 21) $\square_{0}$ No (Skip to Question 25)

\begin{tabular}{|c|c|c|c|c|c|c|}
\hline \multirow[t]{2}{*}{$\begin{array}{l}\text { 21. How comfortable do you feel when } \\
\text { bicycling in the Dearborn Street protectec } \\
\text { bike lanes? }\end{array}$} & \begin{tabular}{|c:} 
Very \\
Uncomfortable \\
$(1)$
\end{tabular} & (2) & (3) & (4) & (5) & $\begin{array}{l}\text { Very } \\
\text { Comfortable } \\
(6)\end{array}$ \\
\hline & $\square_{1}$ & $\square_{2}$ & $\square_{3}$ & $\square_{4}$ & $\square_{5}$ & $\square_{6}$ \\
\hline
\end{tabular}

22. Because of the Dearborn Street protected bike lanes...

\begin{tabular}{|c|c|c|c|c|c|c|}
\hline & $\begin{array}{l}\text { Decreased } \\
\text { a Lot }\end{array}$ & $\begin{array}{l}\text { Decreased } \\
\text { Somewhat }\end{array}$ & $\begin{array}{c}\text { Not } \\
\text { Changed }\end{array}$ & $\begin{array}{l}\text { Increased } \\
\text { Somewhat }\end{array}$ & $\begin{array}{c}\text { Increased } \\
\text { a Lot }\end{array}$ & $\begin{array}{l}\text { No } \\
\text { Opinion }\end{array}$ \\
\hline $\begin{array}{l}\text { the likelihood that I will choose to bicycle on } \\
\text { Dearborn St. as opposed to other streets has... }\end{array}$ & $\square_{1}$ & $\square_{2}$ & $\square_{3}$ & $\square_{4}$ & $\square_{5}$ & $\square_{9}$ \\
\hline $\begin{array}{r}\text { the time it takes me to bicycle on Dearborn Street } \\
\text { has... }\end{array}$ & $\square_{1}$ & $\square_{2}$ & $\square_{3}$ & $\square_{4}$ & $\square_{5}$ & $\square_{9}$ \\
\hline $\begin{array}{r}\text { the usefulness of Dearborn Street for getting } \\
\text { places I want to go has... }\end{array}$ & $\square_{1}$ & $\square_{2}$ & $\square_{3}$ & $\square_{4}$ & $\square_{5}$ & $\square_{9}$ \\
\hline $\begin{array}{r}\text { how often I stop at shops and businesses } \\
\text { on Dearborn Street has... }\end{array}$ & $\square_{1}$ & $\square_{2}$ & $\square_{3}$ & $\square_{4}$ & $\square_{5}$ & $\square_{9}$ \\
\hline $\begin{array}{r}\text { drivers' awareness of bicyclists on Dearborn } \\
\text { Street has ... }\end{array}$ & $\square_{1}$ & $\square_{2}$ & $\square_{3}$ & $\square_{4}$ & $\square_{5}$ & $\square_{9}$ \\
\hline drivers' speeds on Dearborn Street have ... & $\square_{1}$ & $\square_{2}$ & $\square_{3}$ & $\square_{4}$ & $\square_{5}$ & $\square_{9}$ \\
\hline $\begin{array}{l}\text { how comfortable I feel when bicycling } \\
\text { on Dearborn Street has... }\end{array}$ & $\square_{1}$ & $\square_{2}$ & $\square_{3}$ & $\square_{4}$ & $\square_{5}$ & $\square_{9}$ \\
\hline how often I ride a bicycle overall has ... & $\square_{1}$ & $\square_{2}$ & $\square_{3}$ & $\square_{4}$ & $\square_{5}$ & $\square_{9}$ \\
\hline $\begin{array}{l}\text { the difficulty of making a right-turn off of } \\
\text { Dearborn St. while bicycling Northbound has ... }\end{array}$ & $\square_{1}$ & $\square_{2}$ & $\square_{3}$ & $\square_{4}$ & $\square_{5}$ & $\square_{9}$ \\
\hline $\begin{array}{l}\text { the difficulty of navigating around } \\
\text { turning motor vehicles has... }\end{array}$ & $\square_{1}$ & $\square_{2}$ & $\square_{3}$ & $\square_{4}$ & $\square_{5}$ & $\square_{9}$ \\
\hline $\begin{array}{r}\text { the difficulty of navigating around } \\
\text { pedestrians has... }\end{array}$ & $\square_{1}$ & $\square_{2}$ & $\square_{3}$ & $\square_{4}$ & $\square_{5}$ & $\square_{9}$ \\
\hline
\end{tabular}

23. In the past 30 days, how many days have you bicycled for transportation (i.e. not just for recreation/ exercise)?

24. Did you fill out a separate online bicyclist survey about the Dearborn Street protected bike lanes from us recently?
$\square_{1}$ Yes
$\square_{0}$ No 
About Walking on Dearborn Street

25. Have you walked on this section of Dearborn Street since the protected bike lanes were built?

$\square_{1}$ Yes (Proceed to Question 26) $\quad \square_{0}$ No (Skip to Question 29)

26. Because of the Dearborn Street protected bike lanes....

drivers' speeds on Dearborn St. have generally ...

the number of bicyclists riding

on the sidewalk has. .

my satisfaction with the walking environment on Dearborn Street has.

my sense of safety when crossing Dearborn St. has ...

27. These "Look Bikes" pavement markings were added at some crosswalks:

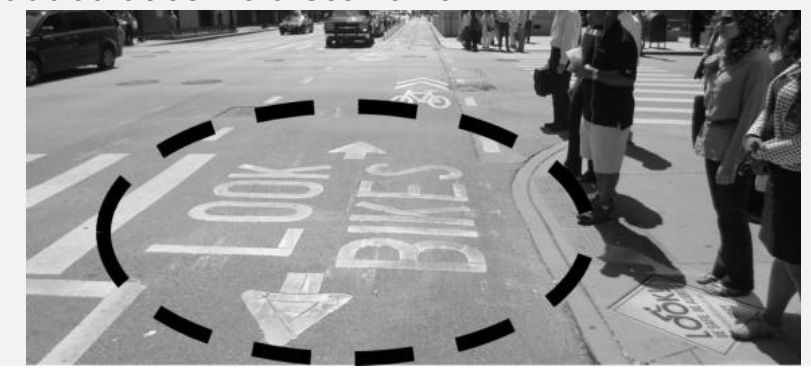

How effective do you think these markings will be at warning pedestrians about bicycle traffic?

\begin{tabular}{|c:c:c:c:c:c|}
\hline $\begin{array}{c}\text { Not effective } \\
\text { at all } \\
\text { (1) }\end{array}$ & (2) & (3) & (4) & (5) & (6) \\
\hline & & & & & \\
$\square_{1}$ & $\square_{2}$ & $\square_{3}$ & $\square_{4}$ & $\square_{5}$ & $\square_{6}$ \\
& & & & & \\
\hline
\end{tabular}

28. Do you ever walk or jog in the protected bike lanes, rather than on the sidewalk?

$\square_{0}$ No

$\square_{1}$ Yes (Please explain why you choose the bikelane over the sidewalk in the space to the right) $\rightarrow$

About Visiting Businesses on Dearborn Street

29. How many days per month do you spend money at businesses on or near Dearborn Street? days

30. How did you travel to get there?

(Enter 0 if None)

a Lot :Opinion

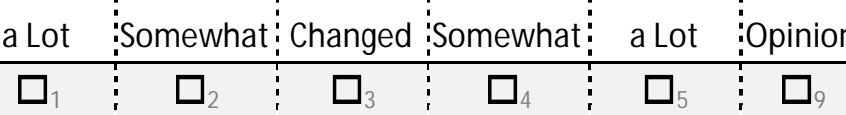

$\square_{1} \square$

$\square$

$\square$


34. What is the best thing about the protected bike lanes?
35. What is the worst thing about the protected bike lanes?

\section{About You}

We have a few questions about you so that we may understand the characteristics of our survey respondents. We will keep this information confidential and it is not linked to your name
36. Do you have a current:
Driver's license
Transit pass
Bikeshare (Divvy) Membership
Carshare M embership

37. How many working motor vehicles does your household own or lease?

(Do not include motorhomes)

$$
\text { \#Vehicles }
$$

(Enter $\overline{0}$ if $\overline{\text { None}}$ )

38. How many working adult bicycles does your household own?

$$
\begin{aligned}
& \text { \#Bicycles } \\
& \text { (Enter } 0 \text { if } \\
& \text { None) }
\end{aligned}
$$

39. INCLUDING YOURSELF, how many people live in your household?

$$
\begin{aligned}
& \text { \#Adults } \\
& \text { \#Children } \\
& \begin{array}{l}
\text { (Enter } 0 \text { if } \\
\text { if } \\
\text { None })
\end{array}
\end{aligned}
$$

40. How long have you lived at this home?

$$
\text { \#Years }
$$

41. Do you rent or own your home?

$$
\square_{1} \text { Rent } \quad \square_{2} \text { Own } \quad \square_{3} \text { Other }
$$

42. Do you consider yourself:

(Select all that apply)

$\square$ American Indian or Alaska Native
$\square$ Asian
$\square$ Black or African American
$\square$ Hispanic or Latino/a
$\square$ White or Caucasian
$\square$ Other:

43. What is your age? years

44. What is your gender?
$\square_{1}$ Male
$\square_{2}$ Female

45. What is your employment status?

(Select all that apply)

$\square$ I work outside the home-> What is the zip code of your place of work?

$\square$ I go to school outside the home->What is the zip code of your school?

$\square$ I work from home

$\square$ Not employed at this time

46. What is your annual household income?
$\square_{1}$ Less than $\$ 25,000$
$\square, \$ 25,000$ to less than $\$ 50,000$
$\square_{3} \$ 50,000$ to less than $\$ 75,000$
$\square_{4} \$ 75,000$ to less than $\$ 100,000$
$\square_{5} \$ 100,000$ to less than $\$ 200,000$
$\square_{6} \$ 200,000$ or more
$\square_{9}$ I prefer not to provide this information

47. What is the highest level of school you have completed?
$\square_{1}$ Some high school or less
$\square_{2}$ High school diploma or GED
$\square_{3}$ Some College
$\square$ Trade/Vocational School
$\square_{5}$ Associate Degree
$\square$ Four-year college degree or more
$\square$ Other:

Thank you for taking our survey! If you would like to be entered in a random drawing for one of three $\$ 100$ Amazon gift cards please enter your name and contact information on the raffle slip you received with the survey.

48. Is there anything else you'd like to tell us? (Use the space below) 


\section{Resident: Portland - NE Multnomah ST.}




\section{Neighborhood Street Study}

About How You Get Around
(1) Complete this paper survey and the enclosed raffle slip.

Return in the postage-paid envelope;

\section{$\underline{\text { OR }}$}

(2) Complete the online survey and raffle entry at: http:// tinyurl.com/ PDXstreetstudy To take the online survey, you will need to enter this code: P0019

Survey deadline 11/ 15/ 2013

\begin{tabular}{|c|c|c|c|c|c|c|}
\hline \multirow{2}{*}{$\begin{array}{l}\text { 1. For each mode of transportation, } \\
\text { please indicate your level of use: }\end{array}$} & \multicolumn{3}{|c|}{$\begin{array}{c}\text { Commute Trips } \\
\text { (to/from work or school) }\end{array}$} & \multicolumn{3}{|c|}{$\begin{array}{c}\text { Other Trips } \\
\text { (e.g. to the store, park, etc.) }\end{array}$} \\
\hline & Most Trips & Some Tri & No Trips & Most Tri & ome Ti & No Trips \\
\hline Car/truck/motor vehicle (including carpool) & $\square_{1}$ & $\square_{2}$ & $\square_{3}$ & $\square_{1}$ & $\square_{2}$ & $\square_{3}$ \\
\hline Walking & $\square_{1}$ & $\square_{2}$ & $\square_{3}$ & $\square_{1}$ & $\square_{2}$ & $\square_{3}$ \\
\hline Bicycling & $\square_{1}$ & $\square_{2}$ & $\square_{3}$ & $\square_{1}$ & $\square_{2}$ & $\square_{3}$ \\
\hline Public Transportation & $\square_{1}$ & $\square_{2}$ & $\square_{3}$ & $\square_{1}$ & $\square_{2}$ & $\square_{3}$ \\
\hline Other (please specify): & $\square_{1}$ & $\square_{2}$ & $\square_{3}$ & $\square_{1}$ & $\square_{2}$ & $\square_{3}$ \\
\hline
\end{tabular}

2. Compared to two years ago, are you taking more or fewer trips by each mode of transportation?

\begin{tabular}{|c|c:c|}
\hline More Trips & No Change & Fewer Trips \\
\hline$\square_{1}$ & $\square_{2}$ & $\square_{3}$ \\
$\square_{1}$ & $\square_{2}$ & $\square_{3}$ \\
$\square_{1}$ & $\square_{2}$ & $\square_{3}$ \\
$\square_{1}$ & $\square_{2}$ & $\square_{3}$ \\
$\square_{1}$ & $\square_{2}$ & $\square_{3}$ \\
\hline
\end{tabular}

Other (please specify):

Public Transportation

\section{About Your Neighborhood}

\section{Over the past two years, changes to my} neighborhood as a place for ...

\begin{tabular}{|c|c|c|c|c|}
\hline $\begin{array}{c}\text { Very } \\
\text { vegative }\end{array}$ & $\begin{array}{l}\text { Somewhat } \\
\text { Neqative }\end{array}$ & $\begin{array}{l}\text { No Impact/ } \\
\text { No Changes }\end{array}$ & $\begin{array}{l}\text { Somewhat } \\
\text { Positive }\end{array}$ & $\begin{array}{c}\text { Very } \\
\text { Positive }\end{array}$ \\
\hline
\end{tabular}

walking have been

driving have been ...

bicycling have been

taking public transportation have been ...
Walking :

Bicycling

무

$\square_{1}$

$\square_{1}$

西

\section{Overall, my level of satisfaction with transportation} in my neighborhood is...

\begin{tabular}{|c|c:c:c|}
\hline $\begin{array}{c}\text { Very } \\
\text { Dissatisfied }\end{array}$ & $\begin{array}{c}\text { Somewhat } \\
\text { Dissatisfied }\end{array}$ & $\begin{array}{c}\text { Somewhat } \\
\text { Satisfied }\end{array}$ & $\begin{array}{c}\text { Very } \\
\text { Satisfied }\end{array}$ \\
\hline$\square_{1}$ & $\square_{2}$ & $\square_{3}$ & $\square_{4}$ \\
\hline
\end{tabular}

\section{About People You Encounter on the Street}

\section{Please indicate if you agree or disagree with the} following statements:

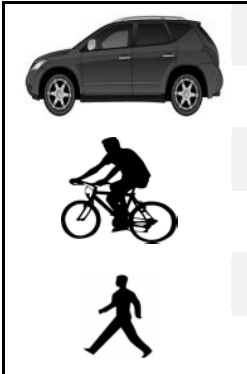

Most drivers follow the rules of the road.

Most drivers are predictable.

Most bicyclists follow the rules of the road.

M ost bicyclists are predictable.

M ost pedestrians follow the rules of the road.

Most pedestrians are predictable.

Strongly Disagree Disagree : Agree 


\begin{tabular}{|c|c|c|c|c|c|}
\hline $\begin{array}{l}\text { 6. Please indicate whether you agree or disagree } \\
\text { with the following statements about bicycling: }\end{array}$ & $\begin{array}{l}\text { Strongly } \\
\text { Disagree }\end{array}$ & $\begin{array}{l}\text { Somewhat } \\
\text { Disagree }\end{array}$ & $\begin{array}{l}\text { Somewhat } \\
\text { Agree }\end{array}$ & $\begin{array}{c}\text { Strongly } \\
\text { Agree }\end{array}$ & $\begin{array}{c}\text { No } \\
\text { Opinion }\end{array}$ \\
\hline Bicycling in my city is a convenient way to get places. & $\mathbf{\square}_{1}$ & $\square_{2}$ & $\square_{3}$ & $\square_{4}$ & $\square_{9}$ \\
\hline Bicycling in my neighborhood is safe. & $\square_{1}$ & $\square_{2}$ & $\square_{3}$ & $\square_{4}$ & $\square_{9}$ \\
\hline $\begin{array}{r}\text { I am familiar with the bicycle lanes and other bicycle } \\
\text { facilities in my neighborhood. }\end{array}$ & $\square_{1}$ & $\square_{2}$ & $\square_{3}$ & $\square_{4}$ & $\square_{9}$ \\
\hline I would like to bicycle more often for transportation. & $\square_{1}$ & $\square_{2}$ & $\square_{3}$ & $\square_{4}$ & $\square_{9}$ \\
\hline $\begin{array}{l}\text { I would be more likely to ride a bicycle if motor vehicles } \\
\text { and bicycles were physically separated by a barrier. }\end{array}$ & $\square_{1}$ & $\square_{2}$ & $\square_{3}$ & $\square_{4}$ & $\square_{9}$ \\
\hline $\begin{array}{l}\text { Facilities that encourage bicycling for transportation are } \\
\text { a good way to improve public health. }\end{array}$ & $\square_{1}$ & $\square_{2}$ & $\square_{3}$ & $\square_{4}$ & $\square_{9}$ \\
\hline
\end{tabular}

\section{Whether or not you currently ride a bicycle, please consider how comfortable you would be riding a bicycle in each place:}

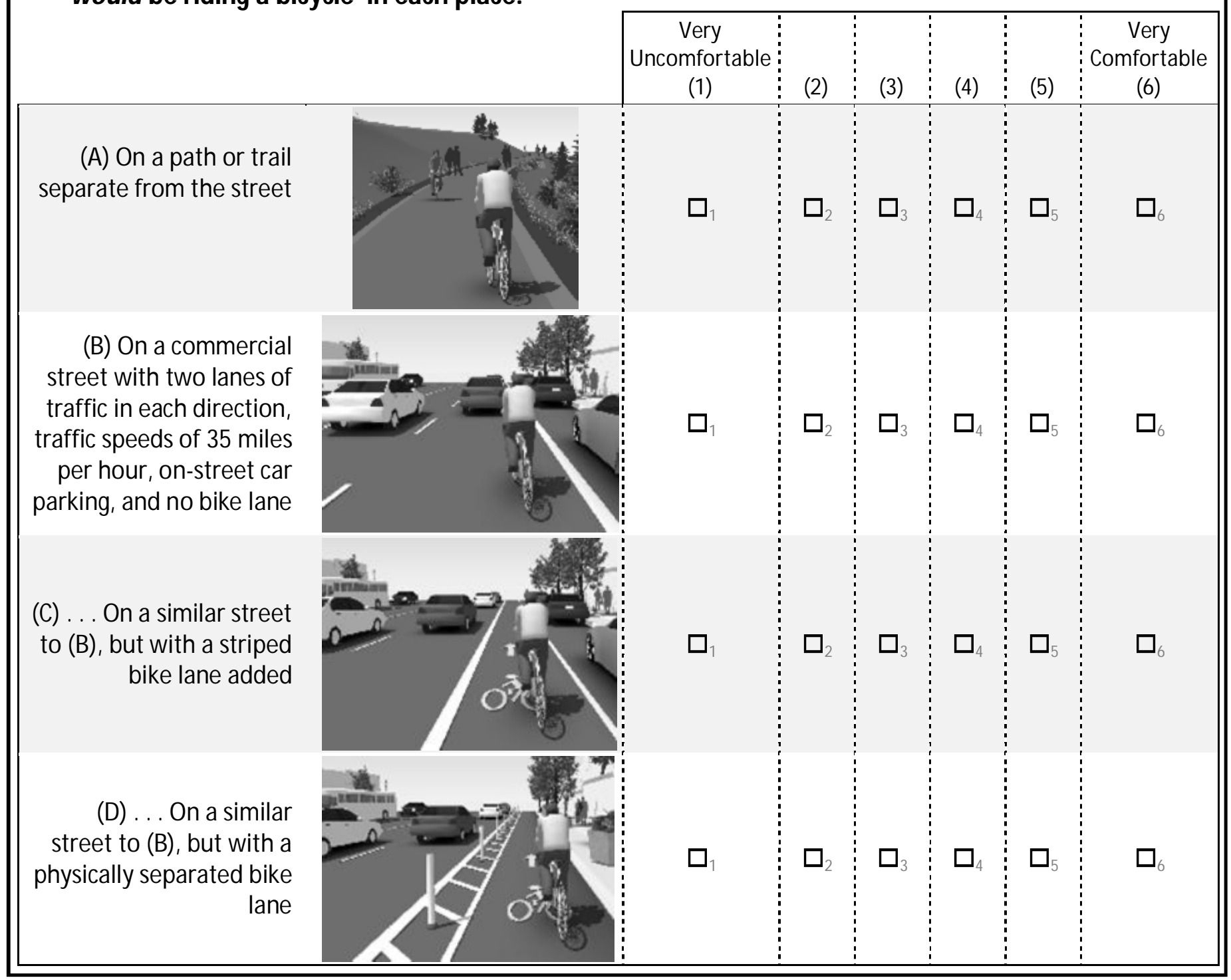




\section{NE M ultnomah Street Protected Bikeway}

A protected bikeway was recently built on NE Multnomah Street from NE Wheeler Avenue to NE 16th Street. In most places, the number of standard traffic lanes was reduced to add a bicycle lane with a painted "buffer" area, plastic "flexposts," and planters that separate the bikeway from the motor vehicle traffic lanes (see pictures below).

NE Multnomah Street before and now:

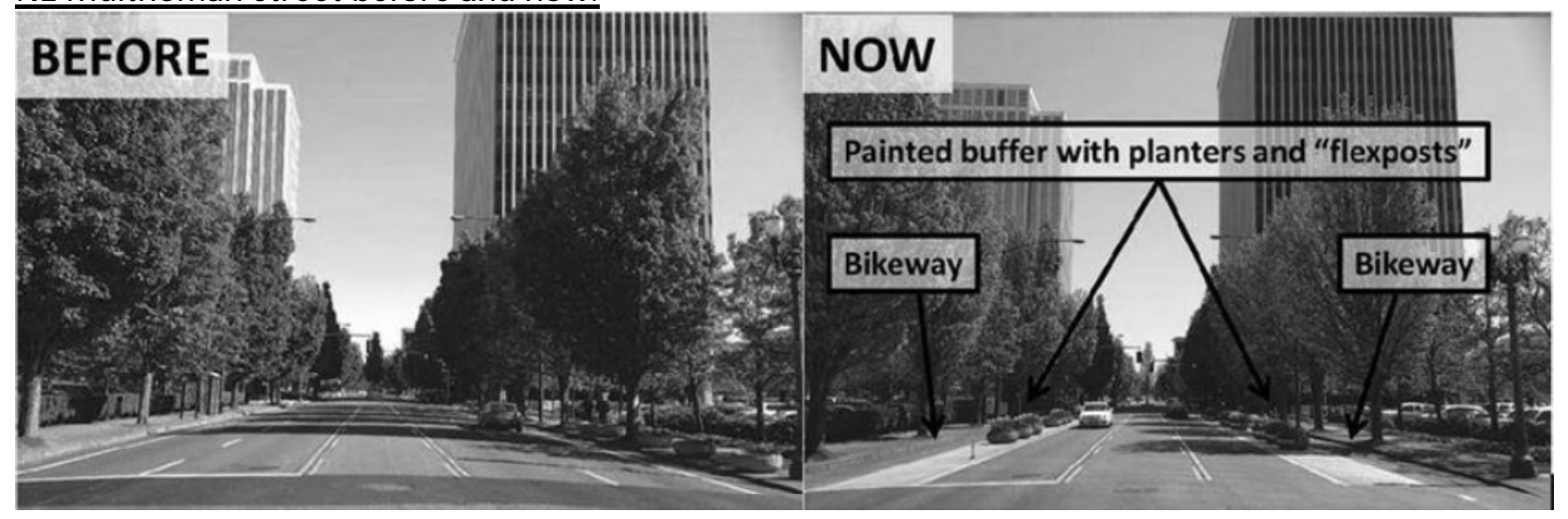

Map of NE M ultnomah protected bikeway:

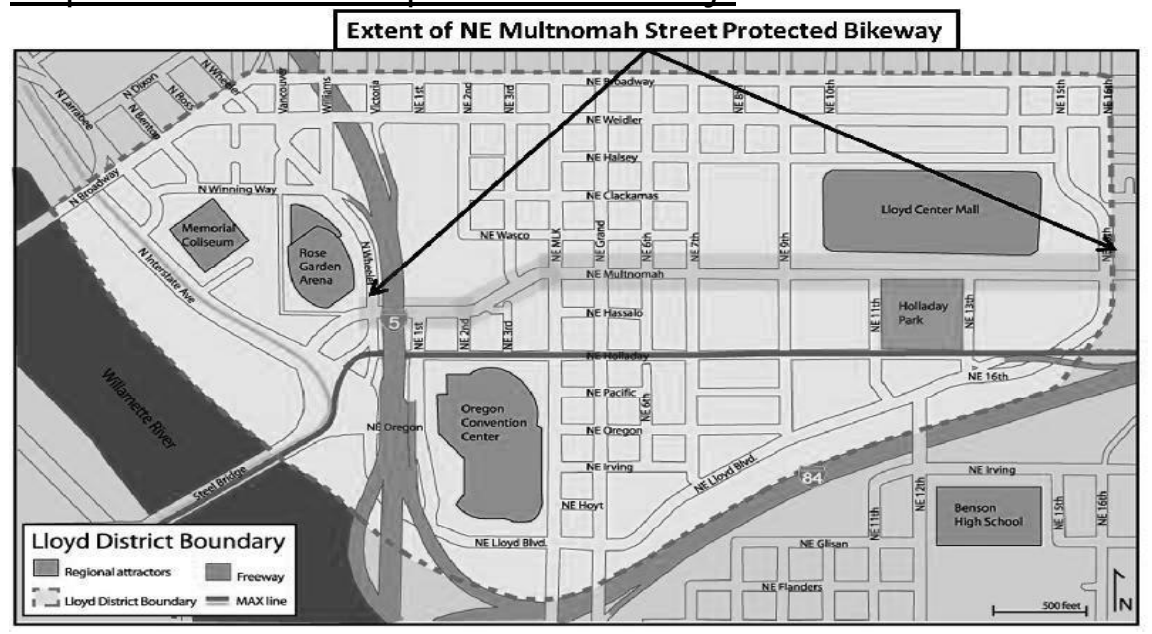

\section{About the NE Multnomah Street Protected Bikeway}

8. Because of the NE Multnomah protected bikeway ...

\begin{tabular}{|c|c|c|c|c|c|c|}
\hline & \begin{tabular}{|c} 
Decreased \\
a Lot
\end{tabular} & $\begin{array}{l}\text { Decreased } \\
\text { Somewhat }\end{array}$ & $\begin{array}{c}\text { Not } \\
\text { Changed }\end{array}$ & $\begin{array}{l}\text { Increased } \\
\text { Somewhat }\end{array}$ & $\begin{array}{l}\text { Increased } \\
\text { a Lot }\end{array}$ & $\begin{array}{c}\text { No } \\
\text { Opinion }\end{array}$ \\
\hline $\begin{array}{l}\text { the desirability of living in my } \\
\text { neighborhood has... }\end{array}$ & $\square_{1}$ & $\square_{2}$ & $\square_{3}$ & $\square_{4}$ & $\square_{5}$ & $\square_{9}$ \\
\hline the aesthetic appeal of NE Multnomah has... & $\square_{1}$ & $\square_{2}$ & $\square_{3}$ & $\square_{4}$ & $\square_{5}$ & $\square_{9}$ \\
\hline the safety of DRIVING on NE M ultnomah has... & $\square_{1}$ & $\square_{2}$ & $\square_{3}$ & $\square_{4}$ & $\square_{5}$ & $\square_{9}$ \\
\hline the safety of BICYCLING on NE Multnomah has. . . & $\square_{1}$ & $\square_{2}$ & $\square_{3}$ & $\square_{4}$ & $\square_{5}$ & $\square_{9}$ \\
\hline the safety of WALKING on NE Multnomah has... & $\square_{1}$ & $\square_{2}$ & $\square_{3}$ & $\square_{4}$ & $\square_{5}$ & $\square_{9}$ \\
\hline $\begin{array}{r}\text { the number of people I see riding bikes } \\
\text { on NE Multnomah has... }\end{array}$ & $\square_{1}$ & $\square_{2}$ & $\square_{3}$ & $\square_{4}$ & $\square_{5}$ & $\square_{9}$ \\
\hline how well the road works for all people has... & $\square_{1}$ & $\square_{2}$ & $\square_{3}$ & $\square_{4}$ & $\square_{5}$ & $\square_{9}$ \\
\hline
\end{tabular}


About the NE Multnomah Street Protected Bikeway, continued.

9. Please indicate whether you agree or disagree with the following statements about the effectiveness of the NE Multnomah protected bikeway:

The protected bike lanes' design makes it clear where cars can be and where the designated bicycle lanes are.

The buffer effectively separates bikes from cars. The buffer does a good job at protecting bikes from cars.

The protected bike lanes improve the predictability of drivers and bicyclists.

The protected bike lanes make it clear where bicyclists and pedestrians should be. The protected bike lanes effectively separate bicyclists from pedestrians.

\begin{tabular}{|c:c:c:c:c|}
\hline $\begin{array}{c}\text { Strongly } \\
\text { Disagree }\end{array}$ & $\begin{array}{c}\text { Somewhat } \\
\text { Disagree }\end{array}$ & $\begin{array}{c}\text { Somewhat } \\
\text { Agree }\end{array}$ & $\begin{array}{c}\text { Strongly } \\
\text { Agree }\end{array}$ & $\begin{array}{c}\text { No } \\
\text { Opinion }\end{array}$ \\
\hline$\square_{1}$ & $\square_{2}$ & $\square_{3}$ & $\square_{4}$ & $\square_{9}$ \\
$\square_{1}$ & $\square_{2}$ & $\square_{3}$ & $\square_{4}$ & $\square_{9}$ \\
$\square_{1}$ & $\square_{2}$ & $\square_{3}$ & $\square_{4}$ & $\square_{9}$ \\
$\square_{1}$ & $\square_{2}$ & $\square_{3}$ & $\square_{4}$ & $\square_{9}$ \\
$\square_{1}$ & $\square_{2}$ & $\square_{3}$ & $\square_{4}$ & $\square_{9}$ \\
$\square_{1}$ & $\square_{2}$ & $\square_{3}$ & $\square_{4}$ & $\square_{9}$ \\
\hline
\end{tabular}

10. What do you think it means when the pavement is painted yellow along the street? What about where it is painted green?

11. Over time, my opinion of the NE Multnomah protected bikeway has

become:

Enter response in this box

\begin{tabular}{|c:c:c:c:c|}
\hline $\begin{array}{c}\text { A Lot M ore } \\
\text { Negative }\end{array}$ & $\begin{array}{c}\text { Somewhat } \\
\text { More Negative }\end{array}$ & $\begin{array}{c}\text { No } \\
\text { Change }\end{array}$ & $\begin{array}{c}\text { Somewhat } \\
\text { M ore Positive }\end{array}$ & $\begin{array}{c}\text { A Lot } \\
\text { M ore Positive }\end{array}$ \\
\hline$\square_{1}$ & $\square_{2}$ & $\square_{3}$ & $\square_{4}$ & $\square_{5}$ \\
\hline
\end{tabular}

12. Please indicate whether you agree or disagree with the following statements about protected bike lanes.

I would support building more protected bike lanes at other locations.

Overall, I support separating bikes from cars.

\section{About Driving on NE Multnomah Street}

13. Have you driven a motor vehicle on this section of NE Multnomah since the protected bikeway was built?

$\square_{1}$ Yes (Proceed to Question 14)

$\square$ No (Skip to Question 16 on the next page)

14. Since the NE Multnomah protected bikeway was built ...

the number of bicyclists riding in the same lanes with cars on NE Multnomah has...

how safe and predictable bicyclists are acting has ... how safe and predictable drivers are acting has ... the amount of time it takes me to drive on NE Multnomah has...

Decreased Decreased $\quad$ Not Increased Increased a Lot Somewhat:Changed :Somewhat a Lot Opinion

$\begin{array}{c:c:c:c}\square_{1} & \square_{2} & \square_{3} & \square_{4} \\ \square_{1} & \square_{2} & \square_{3} & \square_{4} \\ \square_{1} & \square_{2} & \square_{3} & \square_{4} \\ \square_{1} & \square_{2} & \square_{3} & \square_{4}\end{array}$

\begin{tabular}{c:c:c}
$\square_{5}$ & $\square_{9}$ \\
$\square_{5}$ & $\square_{9}$ \\
$\square_{5}$ & $\square_{9}$ \\
$\square_{5}$ & $\square_{9}$ \\
\hline
\end{tabular}




\section{About Driving on NE M ultnomah Street, continued.}

\begin{tabular}{|c|c|c|c|c|c|}
\hline $\begin{array}{l}\text { 15. The impact of the NE Multnomah protected } \\
\text { bikeway on . . . }\end{array}$ & $\begin{array}{c}\text { Very } \\
\text { Negative }\end{array}$ & $\begin{array}{l}\text { Somewhat } \\
\text { Negative }\end{array}$ & $\begin{array}{l}\text { No Impact/ } \\
\text { Neutral }\end{array}$ & $\begin{array}{l}\text { Somewhat } \\
\text { Positive }\end{array}$ & $\begin{array}{c}\text { Very } \\
\text { Positive }\end{array}$ \\
\hline traffic congestion has been ... & $\square_{1}$ & $\square_{2}$ & $\square_{3}$ & $\square_{4}$ & $\square_{5}$ \\
\hline $\begin{array}{r}\text { my ability to turn off of NE Multnomah at signalized } \\
\text { intersections has been ... }\end{array}$ & $\square_{1}$ & $\square_{2}$ & $\square_{3}$ & $\square_{4}$ & $\square_{5}$ \\
\hline $\begin{array}{r}\text { my ability to turn off of NE Multnomah into driveways and } \\
\text { parking lots has been ... }\end{array}$ & $\square_{1}$ & $\square_{2}$ & $\square_{3}$ & $\square_{4}$ & $\square_{5}$ \\
\hline $\begin{array}{r}\text { my ability to pull onto NE M ultnomah from driveways and } \\
\text { parking lots has been } . .\end{array}$ & $\square_{1}$ & $\square_{2}$ & $\square_{3}$ & $\square_{4}$ & $\square_{5}$ \\
\hline $\begin{array}{r}\text { my ability to find a parking spot on NE Multnomah } \\
\text { has been ... }\end{array}$ & $\square_{1}$ & $\square_{2}$ & $\square_{3}$ & $\square_{4}$ & $\square_{5}$ \\
\hline $\begin{array}{r}\text { how stressful it is to park a car on NE Multnomah } \\
\text { has been ... }\end{array}$ & $\square_{1}$ & $\square_{2}$ & $\square_{3}$ & $\square_{4}$ & $\square_{5}$ \\
\hline
\end{tabular}

16. To make a right turn off of NE M ultnomah, there are different intersection designs:

- At some intersections (PICTURE A) there is a lane where bicyclists and right-turning motorists mix (at these locations the protected bikeway ends prior to the intersection).

- At other intersections (PICTURE B) motorists must turn across the bike lane as they turn right.

\section{Please indicate whether you agree or disagree with the following statements about turning off NE Multnomah in situations like the two shown:}

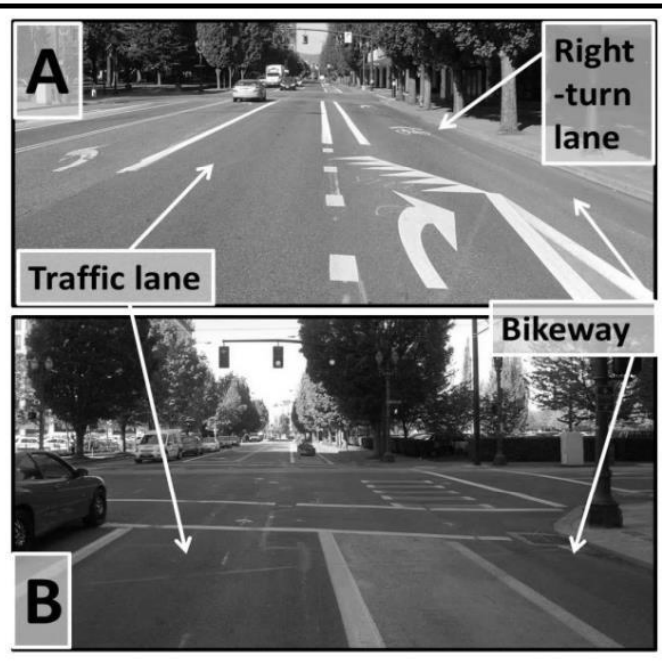

\begin{tabular}{|c|c|c|c|c|c|c|}
\hline & $\begin{array}{l}\text { Strongly } \\
\text { Disagree }\end{array}$ & $\begin{array}{l}\text { Somewhat } \\
\text { Disagree }\end{array}$ & $\begin{array}{l}\text { Somewhat } \\
\text { Agree }\end{array}$ & $\begin{array}{l}\text { Strongly } \\
\text { Agree }\end{array}$ & $\begin{array}{l}\text { I Don't } \\
\text { Know }\end{array}$ & $\begin{array}{c}\text { No } \\
\text { Opinion }\end{array}$ \\
\hline $\begin{array}{l}\text { When drivers want to make a right turn off of NE } \\
\text { Multnomah, it is generally clear where they should } \\
\text { cross over the bike lane. }\end{array}$ & $\square_{1}$ & $\square_{2}$ & $\square_{3}$ & $\square_{4}$ & $\square_{5}$ & $\square_{9}$ \\
\hline $\begin{array}{c}\text { At intersections like Picture } \mathbf{A} \text { above: Before moving } \\
\text { into the right-turn lane, drivers are able to adequately } \\
\text { see any approaching bicyclists in the bike lane. }\end{array}$ & $\square_{1}$ & $\square_{2}$ & $\square_{3}$ & $\square_{4}$ & $\square_{5}$ & $\square_{9}$ \\
\hline $\begin{array}{l}\text { The markings in Picture } \mathbf{A} \text { indicate that motorists } \\
\text { should yield to bicyclists when making a right turn. }\end{array}$ & $\square_{1}$ & $\square_{2}$ & $\square_{3}$ & $\square_{4}$ & $\square_{5}$ & $\square_{9}$ \\
\hline $\begin{array}{r}\text { At intersections like Picture B above: When drivers } \\
\text { want to turn right, they are able to adequately see any } \\
\text { approaching bicyclists in the bike lane. }\end{array}$ & $\square_{1}$ & $\square_{2}$ & $\square_{3}$ & $\square_{4}$ & $\square_{5}$ & $\square_{9}$ \\
\hline $\begin{array}{r}\text { The green dashed lines in the intersection like in Picture } \\
\text { B have made drivers pay closer attention to bicyclists } \\
\text { when turning right. }\end{array}$ & $\square_{1}$ & $\square_{2}$ & $\square_{3}$ & $\square_{4}$ & $\square_{5}$ & $\square_{9}$ \\
\hline
\end{tabular}

\section{Do you avoid driving on NE Multnomah because of the protected bikeway?}


18. Have you bicycled on this section of NE M ultnomah since the protected bikeway was built?

$\square_{1}$ Yes (Proceed to Question 19) $\square_{0}$ No (Skip to Question 23)

19. How comfortable do you feel when bicycling in the NE Multnomah protected bikeway?

\begin{tabular}{|c|c|c|c|c|c|}
\hline $\begin{array}{l}\text { Very } \\
\text { Uncomfortable } \\
\text { (1) }\end{array}$ & (2) & (3) & (4) & (5) & $\begin{array}{c}\text { Very } \\
\text { Comfortable } \\
\text { (6) }\end{array}$ \\
\hline$\square_{1}$ & $\square_{2}$ & $\square_{3}$ & 口 & $\square_{5}$ & $\square_{6}$ \\
\hline
\end{tabular}

20. Because of the NE Multnomah protected bikeway ...

\begin{tabular}{|c|c|c|c|c|c|c|}
\hline & $\begin{array}{c}\text { Decrease } \\
\text { a Lot }\end{array}$ & $\begin{array}{l}\text { Decreased } \\
\text { Somewhat }\end{array}$ & $\begin{array}{c}\text { Not } \\
\text { Changed }\end{array}$ & $\begin{array}{l}\text { Increased } \\
\text { Somewhat }\end{array}$ & $\begin{array}{l}\text { Increased } \\
\text { a Lot }\end{array}$ & \begin{tabular}{|c|c} 
No \\
Opinion
\end{tabular} \\
\hline $\begin{array}{l}\text { the likelihood that I will choose to bicycle on } \\
\text { NE M ultnomah as opposed to other streets has. . }\end{array}$ & $\square_{1}$ & $\square_{2}$ & $\square_{3}$ & $\square_{4}$ & $\square_{5}$ & $\square_{9}$ \\
\hline $\begin{array}{r}\text { the time it takes me to bicycle on NE M ultnomah } \\
\text { has... }\end{array}$ & $\square_{1}$ & $\square_{2}$ & $\square_{3}$ & $\square_{4}$ & $\square_{5}$ & $\square_{9}$ \\
\hline $\begin{array}{r}\text { how often I stop at shops and businesses } \\
\text { on NE Multnomah has... }\end{array}$ & $\square_{1}$ & $\square_{2}$ & $\square_{3}$ & $\square_{4}$ & $\square_{5}$ & $\square_{9}$ \\
\hline $\begin{array}{r}\text { drivers' awareness of bicyclists on } \\
\text { NE M ultnomah has ... }\end{array}$ & $\square_{1}$ & $\square_{2}$ & $\square_{3}$ & $\square_{4}$ & $\square_{5}$ & $\square_{9}$ \\
\hline drivers' speeds on NE M ultnomah have ... & $\square_{1}$ & $\square_{2}$ & $\square_{3}$ & $\square_{4}$ & $\square_{5}$ & $\square_{9}$ \\
\hline $\begin{array}{l}\text { how comfortable I feel when bicycling } \\
\text { on NE Multnomah has... }\end{array}$ & $\square_{1}$ & $\square_{2}$ & $\square_{3}$ & $\square_{4}$ & $\square_{5}$ & $\square_{9}$ \\
\hline how often I ride a bicycle overall has... & $\square_{1}$ & $\square_{2}$ & $\square_{3}$ & $\square_{4}$ & $\square_{5}$ & $\square_{9}$ \\
\hline $\begin{array}{l}\text { the difficulty of making a left turn off of NE } \\
\text { Multnomah while bicycling has... }\end{array}$ & $\square_{1}$ & $\square_{2}$ & $\square_{3}$ & $\square_{4}$ & $\square_{5}$ & $\square_{9}$ \\
\hline $\begin{array}{l}\text { the difficulty of navigating around } \\
\text { turning motor vehicles has... }\end{array}$ & $\square_{1}$ & $\square_{2}$ & $\square_{3}$ & $\square_{4}$ & $\square_{5}$ & $\square_{9}$ \\
\hline $\begin{array}{r}\text { the difficulty of navigating around } \\
\text { pedestrians has... }\end{array}$ & $\square_{1}$ & $\square_{2}$ & $\square_{3}$ & $\square_{4}$ & $\square_{5}$ & $\square_{9}$ \\
\hline $\begin{array}{l}\text { drivers' understanding of how to make a right- } \\
\text { turn when bicycles are going straight has... }\end{array}$ & $\square_{1}$ & $\square_{2}$ & $\square_{3}$ & $\square_{4}$ & $\square_{5}$ & $\square_{9}$ \\
\hline
\end{tabular}

21. In the past 30 days, how many days have you bicycled for transportation (i.e. not just for recreation/ exercise)?

22. Did you fill out a separate online bicyclist survey about the NE M ultnomah protected bikeway from us recently?
$\square_{1}$ Yes
$\square_{0}$ No 
23. Have you walked on this section of NE Multnomah since the protected bikeway was built?

$\square_{1}$ Yes (Proceed to Question 24) $\quad \square_{0}$ No (SKip to Question 27)

24. How often do bicyclists in the bikeway stop for pedestrians at unsignalized intersections?

\begin{tabular}{|c:c:c:c|}
\hline Usually & Sometimes & Rarely & Never \\
\hline$\square_{1}$ & $\square_{2}$ & $\square_{3}$ & $\square_{4}$ \\
\hline
\end{tabular}

25. Because of the NE Multnomah protected bikeway . . .

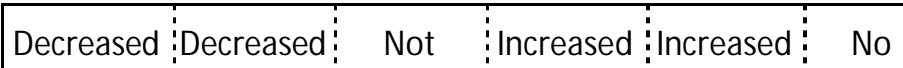
drivers' speeds on NE M ultnomah have generally... the number of bicyclists riding on the sidewalk has... my satisfaction with the walking environment on NE Multnomah has... my sense of safety when crossing NE Multnomah has...

\begin{tabular}{c:c:c:c:c:c}
\hline a Lot & Somewhat & Changed & Somewhat & a Lot & Opinion \\
\hline$\square_{1}$ & $\square_{2}$ & $\square_{3}$ & $\square_{4}$ & $\square_{5}$ & $\square_{9}$ \\
$\square_{1}$ & $\square_{2}$ & $\square_{3}$ & $\square_{4}$ & $\square_{5}$ & $\square_{9}$ \\
$\square_{1}$ & $\square_{2}$ & $\square_{3}$ & $\square_{4}$ & $\square_{5}$ & $\square_{9}$ \\
$\square_{1}$ & $\square_{2}$ & $\square_{3}$ & $\square_{4}$ & $\square_{5}$ & $\square_{9}$ \\
\hline
\end{tabular}

26. Do you ever walk or jog in the protected bikeway, rather than on the sidewalk?
$\square_{0}$ No
$\square_{1}$ Yes (Please explain why you choose the bikeway over the sidewalk in the space to the right) $\rightarrow$

\section{About Visiting Businesses on NE Multnomah Street}

\section{How many days per month do you spend money}

at businesses on or near NE Multnomah?

28. How did you travel to get there?

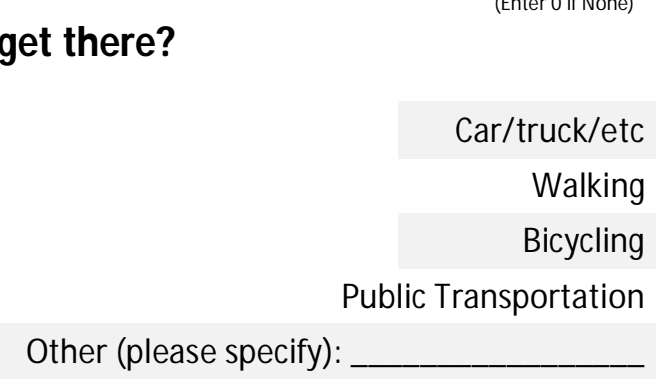
days

\begin{tabular}{|c:c:c|}
\hline $\begin{array}{c}\text { Most of the } \\
\text { time }\end{array}$ & $\begin{array}{c}\text { Some of the } \\
\text { time }\end{array}$ & Never \\
\hline$\square_{1}$ & $\square_{2}$ & $\square_{3}$ \\
$\square_{1}$ & $\square_{2}$ & $\square_{3}$ \\
$\square_{1}$ & $\square_{2}$ & $\square_{3}$ \\
$\square_{1}$ & $\square_{2}$ & $\square_{3}$ \\
$\square_{1}$ & $\square_{2}$ & $\square_{3}$ \\
\hline
\end{tabular}

29. What types of businesses in the area do you patronize? (check all that apply)
$\square_{1}$ Retail Shops
$\square_{2}$ Banks
$\square_{3}$ Restaurants
$\square_{4}$ Bars
$\square_{5}$ Coffee Shop
$\square_{6}$ Other ->

30. When you visit this area, how many businesses do you typically go to each time? (select the best response)

$\square_{1} 1$

$\square_{2} 2$

$\square_{3} 3$ or more

31. Since the protected bikeway was built, are you more or less likely to visit a business on NE Multnomah?
$\square_{1}$ A lot less likely
$\square_{2}$ Somewhat less likely
$\square_{3}$ No change
$\square_{4}$ Somewhat more likely
$\square_{5}$ A lot more likely 
32. What is the best thing about the protected bikeway?
33. What is the worst thing about the protected bikeway?

\section{About You}

We have a few questions about you so that we may understand the characteristics of our survey respondents. We will keep this information confidential and it is not linked to your name

34. Do you have a current: Yes No

$$
\begin{array}{r}
\text { Driver's license } \\
\text { Transit pass } \\
\text { Carshare M embership }
\end{array}
$$

35. How many working motor vehicles does your household own or lease?

(Do not include motorhomes)

$$
\begin{aligned}
& \text { \# Vehicles } \\
& \text { (Enter oif } \text { if } \text { one-) }
\end{aligned}
$$

36. How many working adult bicycles does your household own?

$$
\begin{aligned}
& \text { \#Bicycles } \\
& \text { (Enter oif None- })
\end{aligned}
$$

37. INCLUDING YOURSELF, how many people live in your household?

$$
\begin{aligned}
& \text { \#Adults } \\
& \text { \#Children } \\
& \text { (Enter o oif None-) }
\end{aligned}
$$

38. How long have you lived at this home?

$$
\text { \#Years }
$$

39. Do you rent or own your home?

$$
\square_{1} \text { Rent } \quad \square_{2} \text { Own } \quad \square_{3} \text { Other }
$$

40. Do you consider yourself:

(Select all that apply)

$\square$ American Indian or Alaska Native
$\square$ Asian
$\square$ Black or African American
$\square$ Hispanic or Latino/a
$\square$ White or Caucasian
$\square$ Other:

41. What is your age? years

42. What is your gender?
$\square_{1}$ Male
$\square_{2}$ Female

43. What is your employment status? (Select all that apply)

$\square$ I work outside the home-> What is the zip code of your place of work?

$\square$ I go to school outside the home-> What is the zip code of your school?

$\square$ I work from home

$\square$ Not employed at this time

44. What is your annual household income?
$\square_{1}$ Less than $\$ 25,000$
$\square_{2} \$ 25,000$ to less than $\$ 50,000$
$\square_{3} \$ 50,000$ to less than $\$ 75,000$
$\square_{4} \$ 75,000$ to less than $\$ 100,000$
$\square$ $\$ 100,000$ to less than $\$ 200,000$
$\square \$ 200,000$ or more
$\square_{9}$ I prefer not to provide this information

45. What is the highest level of school you have completed?
$\square_{1}$ Some high school or less
$\square_{2}$ High school diploma or GED
$\square_{3}$ Some College
$\square_{4}$ Trade/Vocational School
$\square_{5}$ Associate Degree
$\square_{6}$ Four-year college degree or more
$\square_{9}$ Other:

Thank you for taking our survey! If you would like to be entered in a random drawing for one of three \$100 Amazon gift cards please enter your name and contact information on the raffle slip you received with the survey.

46. Is there anything else you'd like to tell us? (Use the space below) 


\section{RESIDENT: SAN FRANCISCO - OAK AND FELL STREETS}




\section{Neighborhood Street Study}

\section{About How You Get Around}

1. For each mode of transportation, please indicate your level of use:

Car/truck/motor vehicle (including carpool)
Walking
Bicycling
Public Transportation
Other (please specify):

2. Compared to two years ago, are you taking more or fewer trips by each mode of transportation?
Complete this paper survey and the enclosed raffle slip. Return in the postage-paid envelope;

\section{$\underline{\text { OR }}$}

Complete the online survey and raffle entry at:

http://tinyurl.com/ SFStreetStudy

To take the online survey, you will need to enter this code: F00012

Please complete the survey by 10/09/2013

\begin{tabular}{rr|r:r|r|r|}
\hline Car/truck/motor vehicle (including carpool) & $\square_{1}$ & $\square_{2}$ & $\square_{3}$ \\
Walking & $\square_{1}$ & $\square_{2}$ & $\square_{3}$ \\
Bicycling & $\square_{1}$ & $\square_{2}$ & $\square_{3}$ \\
Public Transportation & $\square_{1}$ & $\square_{2}$ & $\square_{3}$ \\
Other (please specify): & $\square_{1}$ & $\square_{2}$ & $\square_{3}$ \\
\hline
\end{tabular}

\section{About Your Neighborhood}

3. Over the past two years, changes to my neighborhood as a place for . . .
Commute Trips

(to/ from work or school) Most Trips $:$ Some Trips $:$ No Trips
Other Trips

$\begin{array}{l:c}\square_{2} & \square_{3} \\ \square_{2} & \square_{3} \\ \square_{2} & \square_{3} \\ \square_{2} & \square_{3} \\ \square_{2} & \square_{3}\end{array}$

\begin{tabular}{|c:c:c|}
\hline \multicolumn{3}{|c|}{$\begin{array}{c}\text { Other Trips } \\
\text { (e.g. to the store, park, etc.) }\end{array}$} \\
\hline M ost Trips & Some Trips & No Trips \\
\hline$\square_{1}$ & $\square_{2}$ & $\square_{3}$ \\
$\square_{1}$ & $\square_{2}$ & $\square_{3}$ \\
$\square_{1}$ & $\square_{2}$ & $\square_{3}$ \\
$\square_{1}$ & $\square_{2}$ & $\square_{3}$ \\
$\square_{1}$ & $\square_{2}$ & $\square_{3}$ \\
\hline
\end{tabular}

(e.g. to the store, park, etc.) ost Tripsi Some Trips : No Trips

\begin{tabular}{|c|c|c|c|c|c|c|}
\hline 3. & $\begin{array}{l}\text { Over the past two years, changes to my } \\
\text { neighborhood as a place for . . . }\end{array}$ & $\begin{array}{c}\text { Very } \\
\text { Negative }\end{array}$ & $\begin{array}{ll}\text { Somewhat } \\
\text { e }\end{array}$ & $\begin{array}{l:l}\text { No Impact/ } \\
\text { No Changes }\end{array}$ & $\begin{array}{l}\text { Somewhat } \\
\text { Positive }\end{array}$ & $\begin{array}{c}\text { Very } \\
\text { Positive }\end{array}$ \\
\hline & walking have been . & $\square_{1}$ & $\square_{2}$ & $\square_{3}$ & $\square_{4}$ & $\square_{5}$ \\
\hline & driving have been . & $\square_{1}$ & $\square_{2}$ & $\square_{3}$ & $\square_{4}$ & $\square_{5}$ \\
\hline & bicycling have been . . & $\square_{1}$ & $\square_{2}$ & $\square_{3}$ & $\square_{4}$ & $\square_{5}$ \\
\hline & taking public transportation have been . . . & $\square_{1}$ & $\square_{2}$ & $\square_{3}$ & $\square_{4}$ & $\square_{5}$ \\
\hline \multirow[t]{2}{*}{4.} & \multirow{2}{*}{\multicolumn{2}{|c|}{$\begin{array}{l}\text { Overall, my level of satisfaction with transportation } \\
\text { in my neighborhood is... }\end{array}$}} & $\begin{array}{c}\text { Very } \\
\text { Dissatisfied }\end{array}$ & $\begin{array}{l}\text { Somewhat } \\
\text { Dissatisfied }\end{array}$ & $\begin{array}{l}\text { Somewhat } \\
\text { Satisfied }\end{array}$ & $\begin{array}{c}\text { Very } \\
\text { Satisfied }\end{array}$ \\
\hline & & & $\square_{1}$ & $\square_{2}$ & $\square_{3}$ & $\square_{4}$ \\
\hline
\end{tabular}

\section{About People You Encounter on the Street}

5. Please indicate if you agree or disagree with the following statements:

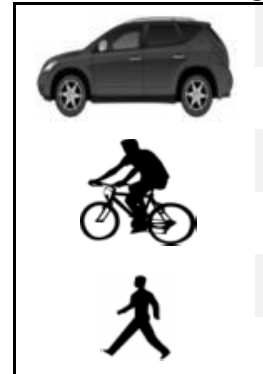

M ost drivers follow the rules of the road.

Most drivers are predictable.

Most bicyclists follow the rules of the road.

Most bicyclists are predictable.

Most pedestrians follow the rules of the road.

Most pedestrians are predictable.
Strongly Somewhat:Somewhat Strongly No

Disagree 
[page intentionally left blank] 


\begin{tabular}{|c|c|c|c|c|c|}
\hline $\begin{array}{l}\text { 6. Please indicate whether you agree or disagree } \\
\text { with the following statements about bicycling: }\end{array}$ & $\begin{array}{l}\text { Strongly } \\
\text { Disagree }\end{array}$ & $\begin{array}{l}\text { Somewhat } \\
\text { Disagree }\end{array}$ & $\begin{array}{l}\text { Somewhat } \\
\text { Agree }\end{array}$ & $\begin{array}{l}\text { Strongly } \\
\text { Agree }\end{array}$ & $\begin{array}{l}\text { No } \\
\text { Opinion }\end{array}$ \\
\hline Bicycling in my city is a convenient way to get places. & $\square_{1}$ & $\square_{2}$ & $\square_{3}$ & $\square_{4}$ & $\square_{9}$ \\
\hline Bicycling in my neighborhood is safe. & $\square_{1}$ & $\square_{2}$ & $\square_{3}$ & $\square_{4}$ & $\square_{9}$ \\
\hline $\begin{array}{r}\text { I am familiar with the bicycle lanes and other bicycle facilities } \\
\text { in my neighborhood. }\end{array}$ & $\square_{1}$ & $\square_{2}$ & $\square_{3}$ & $\square_{4}$ & $\square_{9}$ \\
\hline I would like to bicycle more often for transportation. & $\square_{1}$ & $\square_{2}$ & $\square_{3}$ & $\square_{4}$ & $\square_{9}$ \\
\hline $\begin{array}{l}\text { I would be more likely to ride a bicycle if motor vehicles and } \\
\text { bicycles were physically separated by a barrier. }\end{array}$ & $\square_{1}$ & $\square_{2}$ & $\square_{3}$ & $\square_{4}$ & $\square_{9}$ \\
\hline $\begin{array}{l}\text { Facilities that encourage bicycling for transportation are a } \\
\text { good way to improve public health. }\end{array}$ & $\square_{1}$ & $\square_{2}$ & $\square_{3}$ & $\square_{4}$ & $\square_{9}$ \\
\hline
\end{tabular}

\section{Whether or not you currently ride a bicycle, please consider how comfortable you would be riding a bicycle in each place:}

(B) On a commercial street with two lanes of traffic in each direction, traffic speeds of 35 miles per hour, on-street car parking, and no bike lane

(C) ... On a similar street to (B), but with a striped bike lane added

(D) ... On a similar street to (B), but with a physically separated bike lane

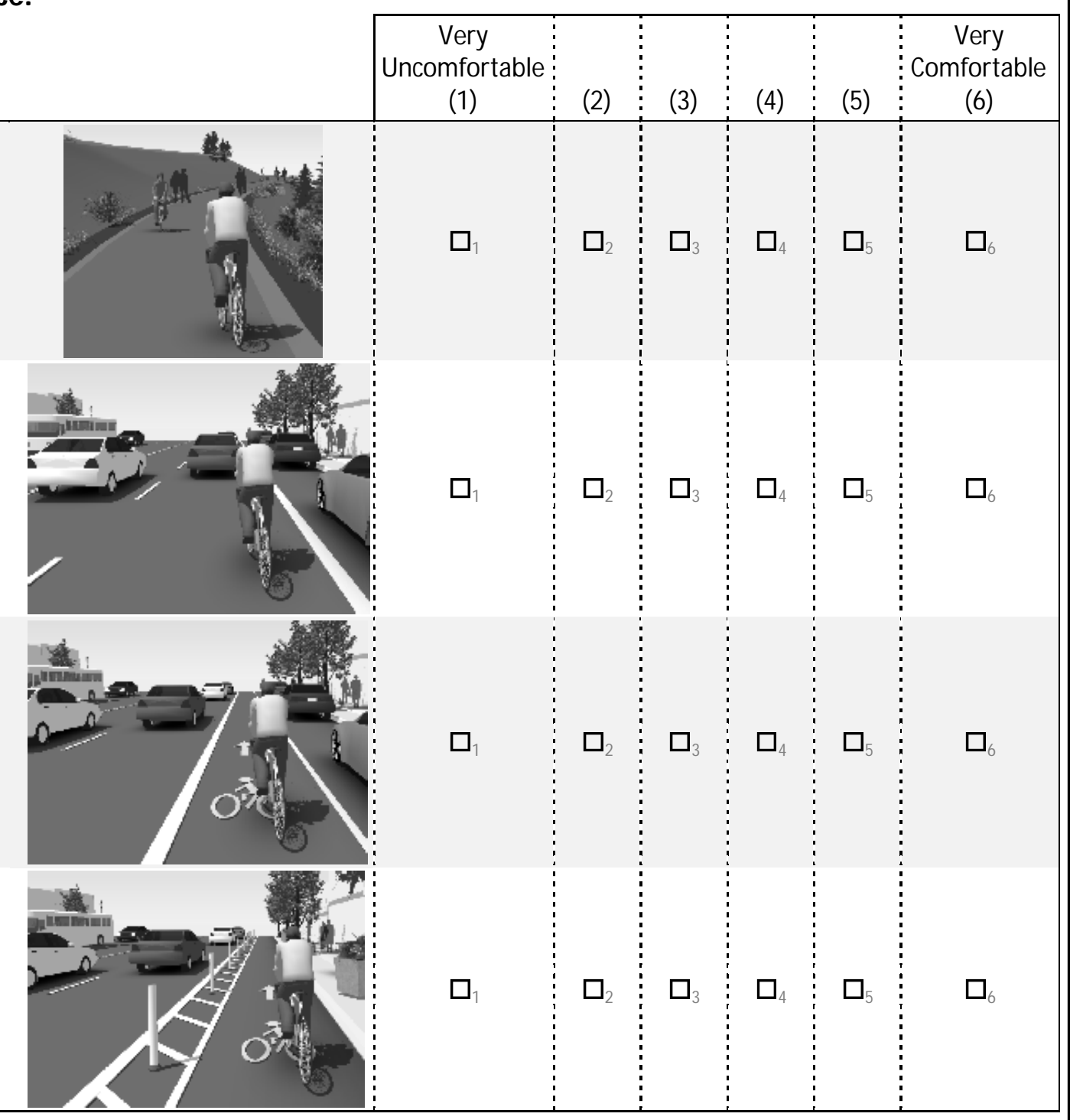




\section{Oak and Fell Street Separated Bikeways}

Separated one-way bikeways (also known as a "cycle tracks") were recently built on Oak and Fell Streets between Baker and Scott Streets (see map below). The bikeways include a painted "buffer" area and plastic "flexposts" separating the separated bikeway from the standard traffic lane (see pictures below). The questions on the following pages are related to these bikeways.

\section{Extent of Oak and Fell Street separated bikeways:}

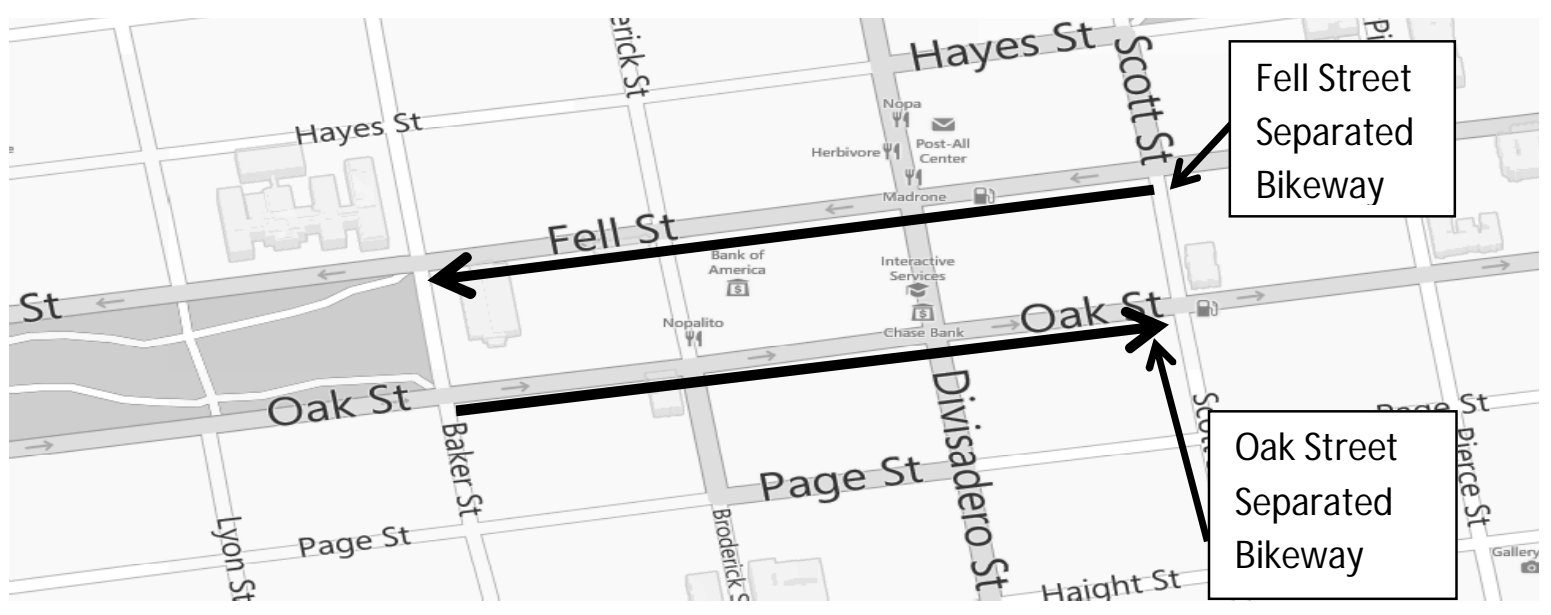

\section{Oak Street:}
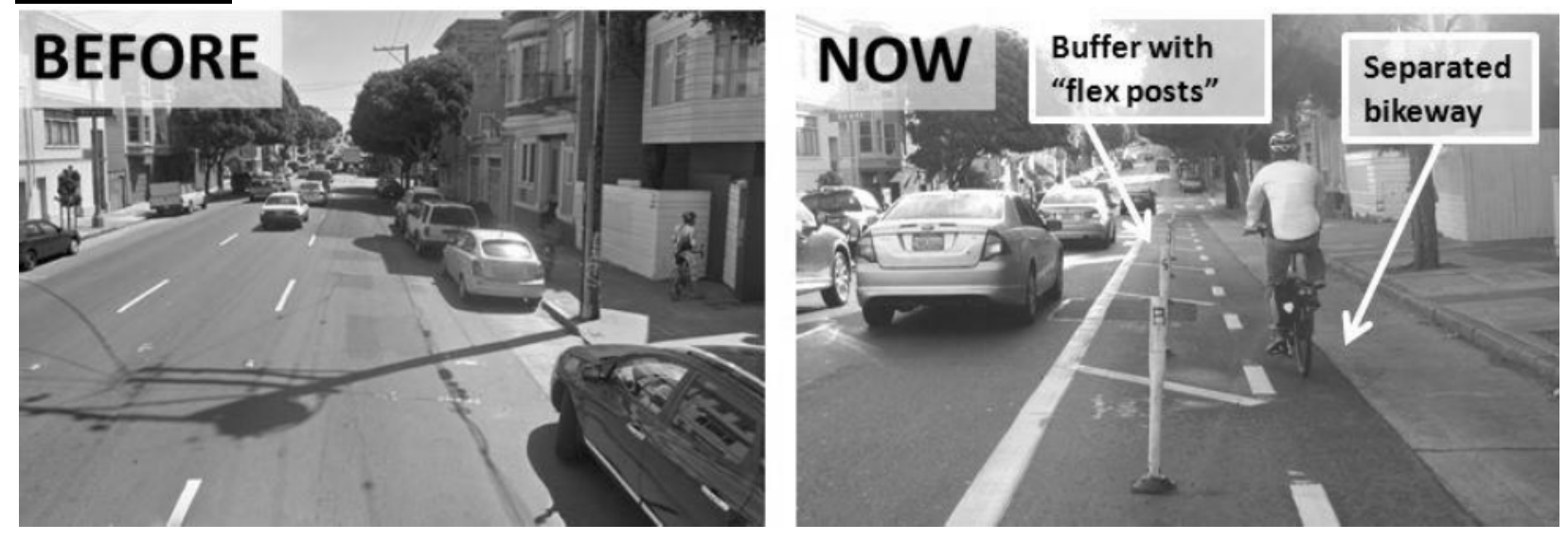

\section{Fell Street:}
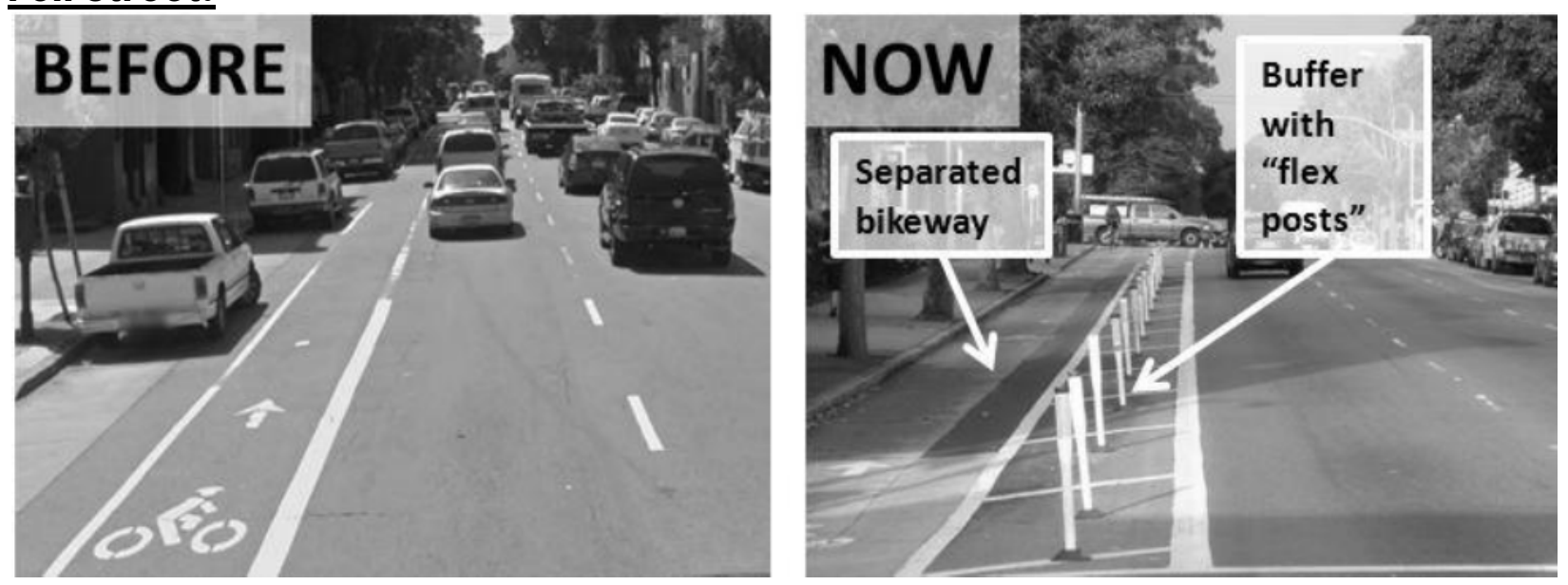
8. Because of the Oak and Fell Street separated bikeways ...

\begin{tabular}{|c|c|c|c|c|c|c|}
\hline & \begin{tabular}{|c|}
$\begin{array}{c}\text { Decreased } \\
\text { a Lot }\end{array}$ \\
\end{tabular} & $\begin{array}{l}\text { Decreased } \\
\text { Somewhat }\end{array}$ & \begin{tabular}{|c|c} 
Not \\
Changed \\
\end{tabular} & $\begin{array}{l}\text { Increased } \\
\text { Somewhat } \\
\end{array}$ & \begin{tabular}{|c} 
Increased \\
a Lot
\end{tabular} & $\begin{array}{c}\text { No } \\
\text { Opinion } \\
\end{array}$ \\
\hline $\begin{array}{l}\text { the desirability of living in my } \\
\text { neighborhood has... }\end{array}$ & $\square_{1}$ & $\mathbf{D}_{2}$ & $\square_{3}$ & $\square_{4}$ & $\square_{5}$ & $\square_{9}$ \\
\hline the aesthetic appeal of Oak and Fell Streets has... & $\square_{1}$ & $\square_{2}$ & $\square_{3}$ & $\square_{4}$ & $\square_{5}$ & $\square_{9}$ \\
\hline the safety of DRIVING on Oak and Fell Streets has. & $\square_{1}$ & $\square_{2}$ & $\square_{3}$ & $\square_{4}$ & $\square_{5}$ & $\square_{9}$ \\
\hline the safety of BICYCLING on Oak and Fell Streets has. . . & $\square_{1}$ & $\square_{2}$ & $\square_{3}$ & $\square_{4}$ & $\square_{5}$ & $\square_{9}$ \\
\hline the safety of WALKING on Oak and Fell Streets has. & $\square_{1}$ & $\square_{2}$ & $\square_{3}$ & $\square_{4}$ & $\square_{5}$ & $\square_{9}$ \\
\hline $\begin{array}{r}\text { the number of people I see riding bicycles } \\
\text { on Oak and Fell Streets has... }\end{array}$ & $\square_{1}$ & $\square_{2}$ & $\square_{3}$ & $\square_{4}$ & $\square_{5}$ & $\mathbf{\square}_{9}$ \\
\hline how well the streets work for all people has ... & $\mathbf{\square}_{1}$ & $\square_{2}$ & $\square_{3}$ & $\square_{4}$ & $\square_{5}$ & $\square_{9}$ \\
\hline
\end{tabular}

9. Please indicate whether you agree or disagree with the following statements about the effectiveness of the Oak and Fell Street separated bikeways:

\begin{tabular}{|c|c|c|c|c|c|}
\hline & $\begin{array}{l}\text { Strongly } \\
\text { Disagree }\end{array}$ & $\begin{array}{l}\text { Somewhat } \\
\text { Disagree }\end{array}$ & $\begin{array}{l}\text { Somewhat } \\
\text { Agree }\end{array}$ & $\begin{array}{c}\text { Strongly } \\
\text { Agree }\end{array}$ & $\begin{array}{c}\text { No } \\
\text { Opinion }\end{array}$ \\
\hline $\begin{array}{r}\text { The design of the bikeways makes it clear where cars can be and } \\
\text { where the designated bicycle lanes are. }\end{array}$ & $\square_{1}$ & $\square_{2}$ & $\square_{3}$ & $\square_{4}$ & $\square_{9}$ \\
\hline The buffer effectively separates bicycles from cars. & $\square_{1}$ & $\square_{2}$ & $\square_{3}$ & $\square_{4}$ & $\square_{9}$ \\
\hline The buffer does a good job at protecting bicycles from cars. & $\square_{1}$ & $\square_{2}$ & $\square_{3}$ & $\square_{4}$ & $\square_{9}$ \\
\hline $\begin{array}{r}\text { The separated bikeways improve the predictability } \\
\text { of drivers and bicyclists. }\end{array}$ & $\square_{1}$ & $\square_{2}$ & $\square_{3}$ & $\square_{4}$ & $\square_{9}$ \\
\hline $\begin{array}{r}\text { The separated bikeways' design makes it clear where } \\
\text { bicyclists and pedestrians should be. }\end{array}$ & $\square_{1}$ & $\square_{2}$ & $\square_{3}$ & $\square_{4}$ & $\square_{9}$ \\
\hline $\begin{array}{l}\text { The separated bikeways effectively } \\
\text { separate bicyclists from pedestrians. }\end{array}$ & $\square_{1}$ & $\square_{2}$ & $\square_{3}$ & $\square_{4}$ & $\square_{9}$ \\
\hline
\end{tabular}

10. Over time, my opinion of the Oak and Fell Street separated bikeways has become:

\begin{tabular}{|c|c:c|c|c|}
\hline $\begin{array}{c}\text { A Lot More } \\
\text { Negative }\end{array}$ & $\begin{array}{c}\text { Somewhat } \\
\text { M ore Negative }\end{array}$ & $\begin{array}{c}\text { Somewhat } \\
\text { No Change }\end{array}$ & $\begin{array}{c}\text { A Lot M ore Positive } \\
\text { Positive }\end{array}$ \\
\hline$\square_{1}$ & $\square_{2}$ & $\square_{3}$ & $\square_{4}$ & $\square_{5}$ \\
\hline
\end{tabular}

11. Please indicate whether you agree or disagree with the following statements about separated bikeways.

\begin{tabular}{|c|c:c:c:c:c|}
\cline { 2 - 6 } \multicolumn{1}{c|}{} & $\begin{array}{c}\text { Strongly } \\
\text { Disagree }\end{array}$ & $\begin{array}{c}\text { Somewhat } \\
\text { Disagree }\end{array}$ & $\begin{array}{c}\text { Somewhat } \\
\text { Agree }\end{array}$ & $\begin{array}{c}\text { Strongly } \\
\text { Agree }\end{array}$ & $\begin{array}{c}\text { No } \\
\text { Opinion }\end{array}$ \\
\hline I would support building more separated bikeways at other & $\square_{1}$ & $\square_{2}$ & $\square_{3}$ & $\square_{4}$ & $\square_{9}$ \\
locations. & $\square_{1}$ & $\square_{2}$ & $\square_{3}$ & $\square_{4}$ & $\square_{9}$ \\
\hline
\end{tabular}




\section{About Intersections on Oak Street}

The following lane and intersection designs were recently installed along Oak Street. Whether or not you are familiar with the locations, please answer the following questions about the designs in the photos below.

\section{When traveling eastbound on Oak Street at the intersection of Broderick Street . . .}

Please indicate whether you think the person should be in lane A or B for each scenario

(select all that apply) $->\quad$ A B

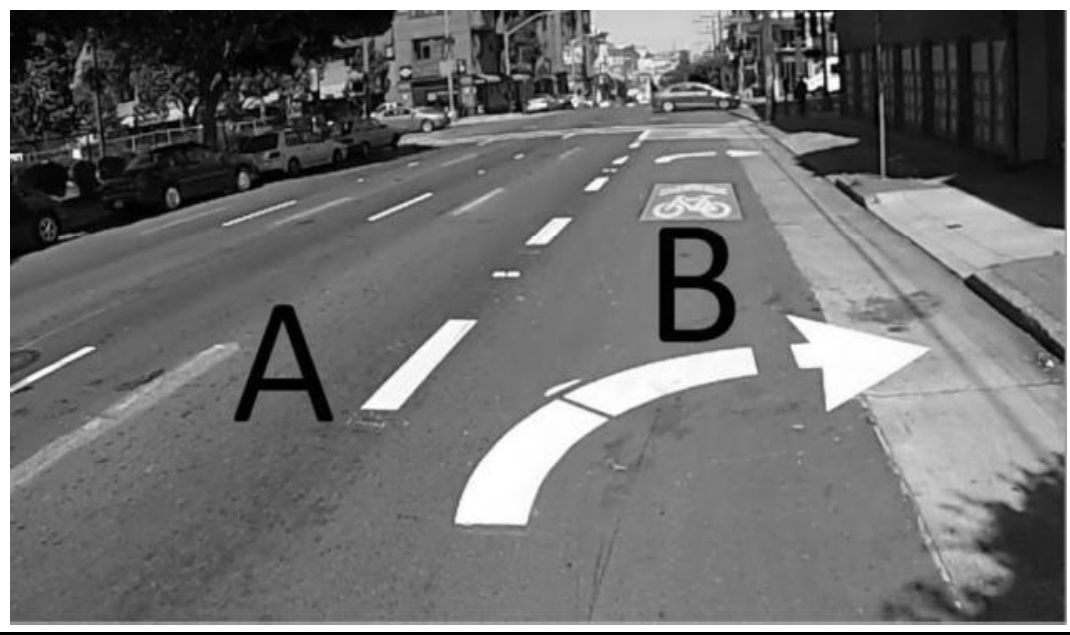

A motorist turning right should be in lane...

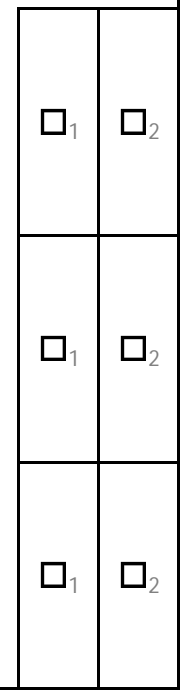

\section{When traveling eastbound on Oak Street at the intersection of Divisadero}

Street . . .

Please indicate whether you think the person should be in lane A, B or C for each scenario

(select all that apply) $\rightarrow \quad$ A $\quad$ B $\quad$ C

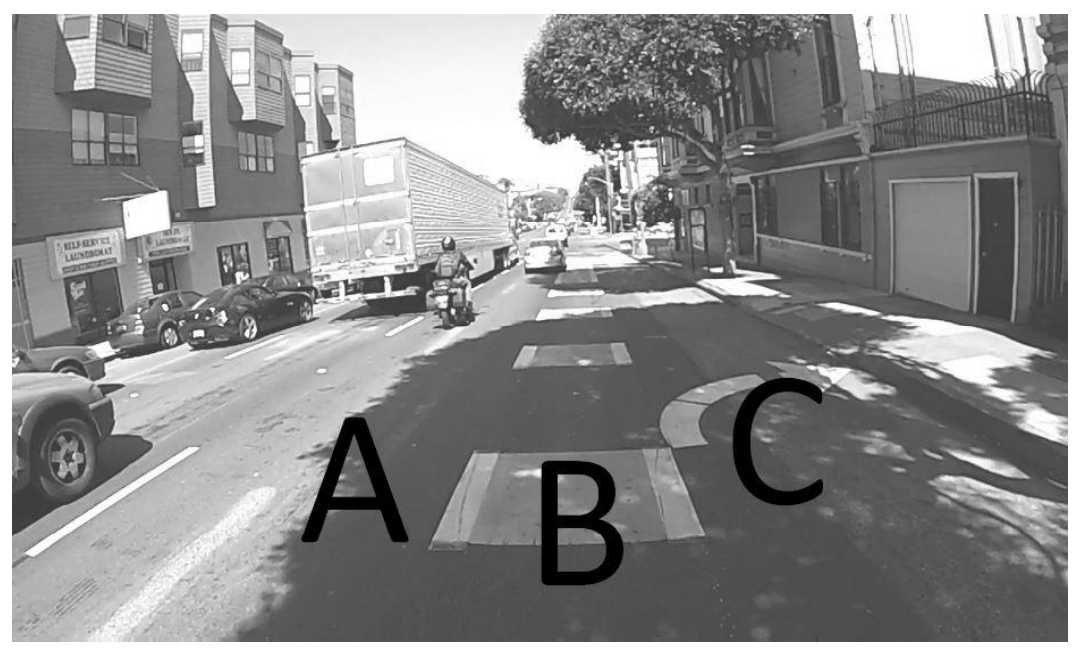

A motorist turning right should be in lane...

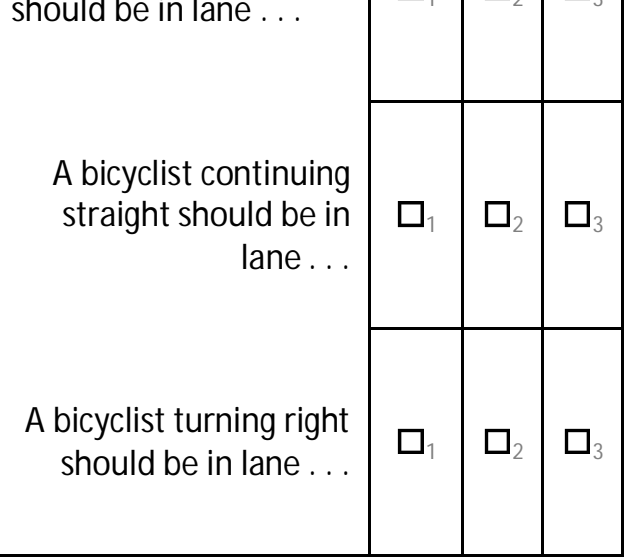

14. Do you think either of the above designs performs better at making clear where drivers and bicyclists should be when approaching the intersection?

$\square_{1}$ The first design is better

$\square_{2}$ The second design is better

$\square_{3}$ Neither one is better 


\section{About Intersections on Fell Street}

The following lane and intersection designs were recently installed along Oak Street. Whether or not you are familiar with the locations, please answer the following questions about the designs in the photos below.

\section{When traveling westbound on Fell Street at the intersection of Baker Street ...}

Please indicate whether you think the person should be in lane A or B for each scenario

(select all that apply) ->

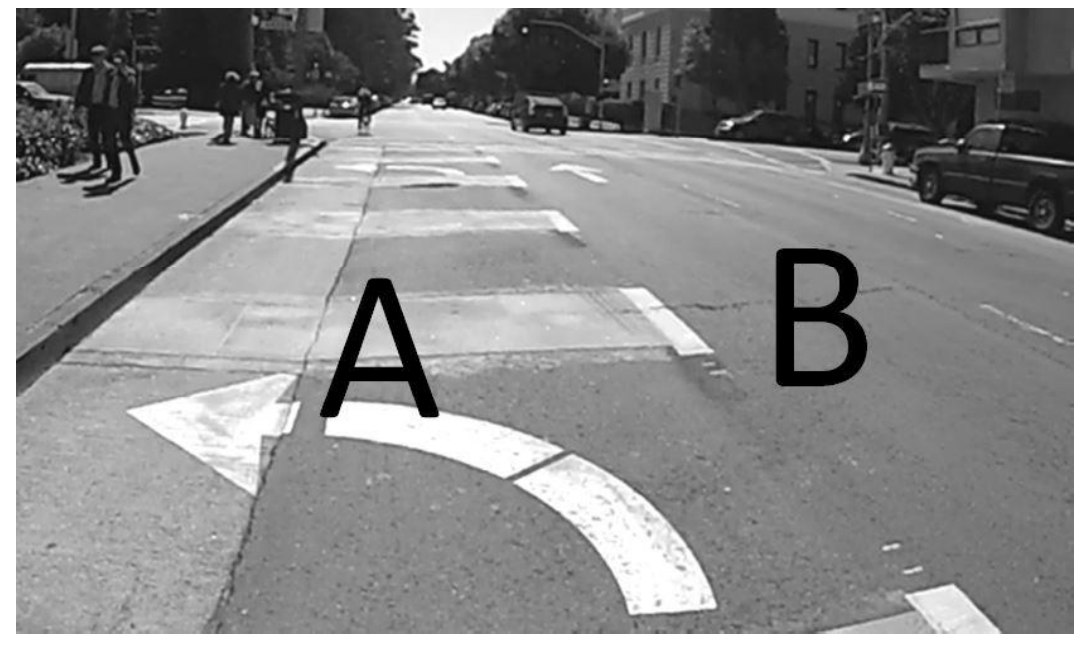

A motorist turning left should be in lane...

A bicyclist continuing straight should be in lane...

A bicyclist turning left should be in lane...

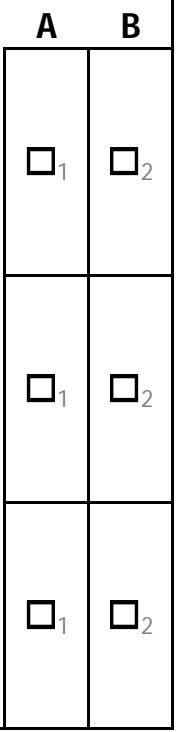

\section{When traveling westbound on Fell Street at the intersection of Divisadero}

\section{Street . . .}

Please indicate whether you think the person should be in lane A, B or C for each scenario (select all that apply) $->\quad$ A $\quad$ B $\quad$ C

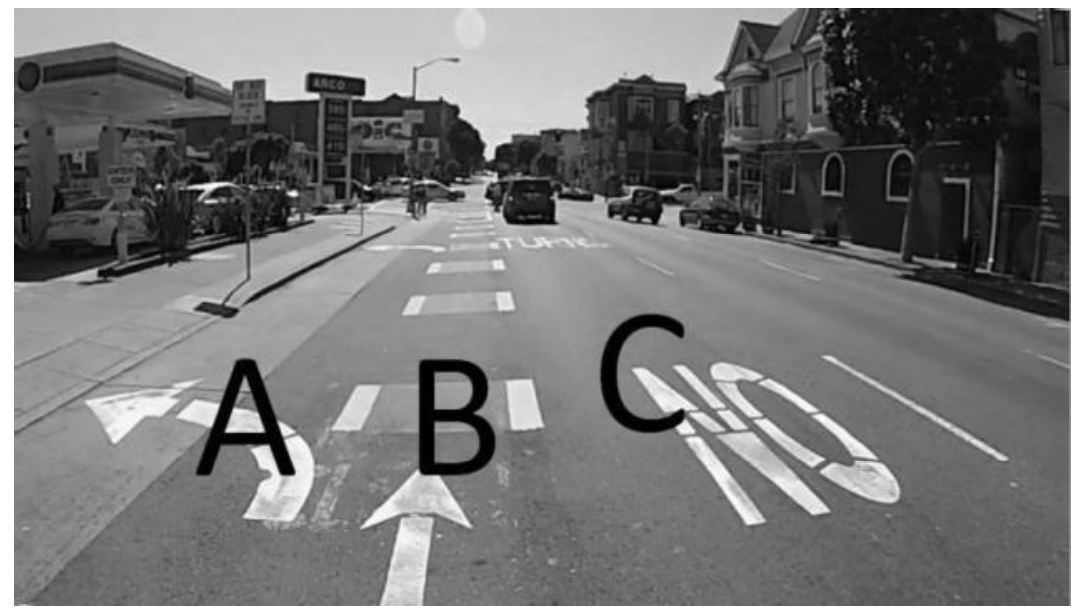

A motorist turning left should be in lane...

A bicyclist continuing straight should be in lane...

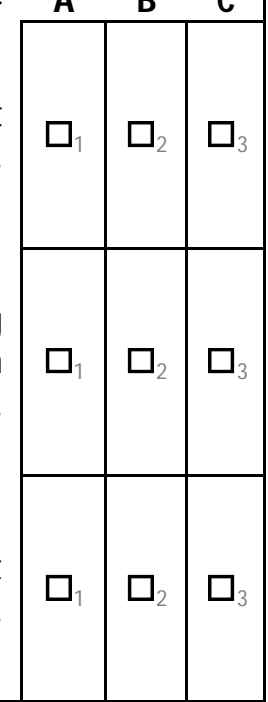

17. Do you think either of the above designs performs better at making clear where drivers and bicyclists should be when approaching the intersection?

$\square_{1}$ The first design is better

$\square_{2}$ The second design is better

Neither one is better

A bicyclist turning left should be in lane... 
18. Is there anything else you would like to tell us about the intersection designs on the prior pages?

Enter response in this box

19. What do you think it means when the pavement is painted green along the bikeway?

Enter response in this box

\section{About Driving on Oak Street and Fell Street}

20. Have you driven a motor vehicle on this section of Oak Street and/ or Fell Street since the separated bikeways were built? (Please refer to map and photos on page 3).

$\square_{1}$ Yes (Proceed to Question 21) $\quad \square_{0}$ No (Skip to Question 26)

21. Consider the intersections presented on pages 5-6. Please indicate whether you agree or disagree with the following statements about the intersection designs:

\begin{tabular}{|c|c|c|c|c|c|}
\hline & $\begin{array}{l}\text { Strongly } \\
\text { Disagree }\end{array}$ & $\begin{array}{l}\text { Somewha } \\
\text { Disagree }\end{array}$ & $\begin{array}{l}\text { Somewhat } \\
\text { Agree }\end{array}$ & $\begin{array}{l}\text { Strongly } \\
\text { Agree }\end{array}$ & $\begin{array}{c}\text { No } \\
\text { Opinion }\end{array}$ \\
\hline $\begin{array}{l}\text { When I want to make a turn off of Oak or Fell Streets across } \\
\text { the bikeway, it is clear where I should cross over the bike lane } \\
\text { to make my turn. }\end{array}$ & $\mathbf{\square}_{1}$ & $\square_{2}$ & $\square_{3}$ & $\square_{4}$ & $\square_{9}$ \\
\hline $\begin{array}{l}\text { When I want to make a turn across the bikeway, I am able to } \\
\text { adequately see if there are any approaching bicyclists. }\end{array}$ & $\square_{1}$ & $\square_{2}$ & $\square_{3}$ & $\square_{4}$ & $\square_{9}$ \\
\hline $\begin{array}{r}\text { The intersection design provides me adequate time/space to } \\
\text { move into the turn-lane }\end{array}$ & $\square_{1}$ & $\square_{2}$ & $\square_{3}$ & $\square_{4}$ & $\square_{9}$ \\
\hline
\end{tabular}


About Driving on Oak Street and Fell Street, continued.

\begin{tabular}{|c|c|c|c|c|c|c|}
\hline & \begin{tabular}{|c|} 
Decreased \\
a Lot \\
\end{tabular} & $\begin{array}{l}\text { Decreased } \\
\text { Somewhat }\end{array}$ & $\begin{array}{c}\text { Not } \\
\text { Changed }\end{array}$ & $\begin{array}{l}\text { Increased } \\
\text { Somewhat }\end{array}$ & $\begin{array}{c}\text { Increased } \\
\text { a Lot }\end{array}$ & $\begin{array}{c}\text { No } \\
\text { Opinion }\end{array}$ \\
\hline how safe and predictable bicydistsare acting has... & $\square_{1}$ & $\square_{2}$ & $\square_{3}$ & $\square_{4}$ & $\square_{5}$ & $\square_{9}$ \\
\hline how safe and predictable criversare acting has... & $\square_{1}$ & $\square_{2}$ & $\square_{3}$ & $\square_{4}$ & $\square_{5}$ & $\square_{9}$ \\
\hline $\begin{array}{l}\text { the number of bicyclists riding in the same lanes with } \\
\text { cars on OakStreet has ... }\end{array}$ & $\square_{1}$ & $\square_{2}$ & $\square_{3}$ & $\square_{4}$ & $\square_{5}$ & $\square_{9}$ \\
\hline $\begin{array}{l}\text { the number of bicyclists riding in the same lanes with } \\
\text { cars on Fell Street has ... }\end{array}$ & $\square_{1}$ & $\square_{2}$ & $\square_{3}$ & $\square_{4}$ & $\square_{5}$ & $\square_{9}$ \\
\hline $\begin{array}{l}\text { the amount of time it takes me to drive } \\
\text { on OakStreet has ... }\end{array}$ & $\square_{1}$ & $\square_{2}$ & $\square_{3}$ & $\square_{4}$ & $\square_{5}$ & $\square_{9}$ \\
\hline $\begin{array}{l}\text { the amount of time it takes me to drive } \\
\text { on Fell Street has... }\end{array}$ & $\square_{1}$ & $\square_{2}$ & $\square_{3}$ & $\square_{4}$ & $\square_{5}$ & $\square_{9}$ \\
\hline
\end{tabular}

23. When driving on Oak and Fell Streets, the impact of the separated bikeways on:

\begin{tabular}{|c|c|c|c|c|c|}
\hline & \begin{tabular}{|c|} 
Very \\
Negative \\
\end{tabular} & $\begin{array}{l}\text { Somewhat } \\
\text { Negative }\end{array}$ & $\begin{array}{l}\text { No Impact/ } \\
\text { Neutral }\end{array}$ & $\begin{array}{l}\text { Somewhat } \\
\text { Positive }\end{array}$ & $\begin{array}{c}\text { Very } \\
\text { Positive }\end{array}$ \\
\hline my ability to find parking on Oak and Fell Streets has been ... & $\square_{1}$ & $\square_{2}$ & $\square_{3}$ & $\square_{4}$ & $\square_{5}$ \\
\hline how stressful it is to park on Oak and Fell Streets has been ... & $\square_{1}$ & $\square_{2}$ & $\square_{3}$ & $\square_{4}$ & $\square_{5}$ \\
\hline $\begin{array}{r}\text { my ability to turn off of Oak and Fell Streets at signalized } \\
\text { intersections has been ... }\end{array}$ & $\square_{1}$ & $\square_{2}$ & $\square_{3}$ & $\square_{4}$ & $\square_{5}$ \\
\hline $\begin{array}{r}\text { my ability to turn off of Oak and Fell Streets into alleys, } \\
\text { driveways, and parking lots has been ... }\end{array}$ & $\square_{1}$ & $\square_{2}$ & $\square_{3}$ & $\square_{4}$ & $\square_{5}$ \\
\hline $\begin{array}{r}\text { my ability to pull onto Oak and Fell Streets from alleys, } \\
\text { driveways, and parking lots has been ... }\end{array}$ & $\square_{1}$ & $\square_{2}$ & $\square_{3}$ & $\square_{4}$ & $\square_{5}$ \\
\hline
\end{tabular}

24. Do you avoid driving on Oak Street because of the separated bikeway?

$\square_{1}$ Yes $\quad \square_{0}$ No

25. Do you avoid driving on Fell Street because of the separated bikeway?

$\square_{1}$ Yes $\quad \square_{0}$ No 
26. Have you bicycled in the Oak or Fell separated bikeways since they were built?

$\square_{1}$ Yes (Proceed to Question 27) $\quad \square_{0}$ No (Skip to Question 32)

\begin{tabular}{|c|c|c|c|c|c|c|}
\hline $\begin{array}{l}\text { 27. How comfortable do you feel when } \\
\text { bicycling in the separated bikeway on }\end{array}$ & \begin{tabular}{|c|} 
Very \\
Uncomfortable \\
(1)
\end{tabular} & (2) & (3) & (4) & (5) & $\begin{array}{c}\text { Very } \\
\text { Comfortable } \\
\text { (6) }\end{array}$ \\
\hline Oak Street & $\mathbf{\square}_{1}$ & $\mathbf{\square}_{2}$ & $\square_{3}$ & $\square_{4}$ & $\square_{5}$ & $\square_{6}$ \\
\hline Fell Street & $\mathbf{\square}_{1}$ & $\square_{2}$ & $\square_{3}$ & $\square_{4}$ & $\square_{5}$ & $\square_{6}$ \\
\hline
\end{tabular}

28. Because of the Oak Street separated bikeway ...
Decreased :Decreased: Not Increased Increased No a Lot Somewhat: Changed Somewhat a Lot Opinion

the likelihood that I will choose to bicycle on

Oak Street as opposed to other streets has. . how often I stop at businesses on Oak Street has... drivers' awareness of bicyclists on Oak Street has . how comfortable I feel bicycling on Oak Street has ... the difficulty of making a left-turn off of Oak Street while bicycling has. .

the difficulty of navigating around turning motor vehicles has... how often I ride a bicycle overall has ...

$\begin{array}{l:l:l:l}\square_{1} & \square_{2} & \square_{3} \\ \square_{1} & \square_{2} & \square_{3} \\ \square_{1} & \square_{2} & \square_{3} \\ \square_{1} & \square_{2} & \square_{3} \\ \square_{1} & \square_{2} & \square_{3} \\ \square_{1} & \square_{2} & \square_{3} \\ \square_{1} & \square_{2} & \square_{3}\end{array}$

$\square_{4}$
$\square_{4}$
$\square_{4}$
$\square_{4}$
$\square_{4}$
$\square_{4}$
$\square_{4}$

\begin{tabular}{l:l|}
$\square_{5}$ & $\square_{9}$ \\
$\square_{5}$ & $\square_{9}$ \\
$\square_{5}$ & $\square_{9}$ \\
$\square_{5}$ & $\square_{9}$ \\
$\square_{5}$ & $\square_{9}$ \\
$\square_{5}$ & $\square_{9}$ \\
$\square_{5}$ & $\square_{9}$ \\
\hline
\end{tabular}

\section{Because of the Fell Street} separated bikeway...

\section{Decreased Decreased Not Increased Increased No a Lot Somewhat: Changed Somewhat a Lot Opinion}

the likelihood that I will choose to bicycle on

Fell Street as opposed to other streets has

how often I stop at businesses on Fell Street has...

drivers' awareness of bicyclists on Fell Street has . . .

how comfortable I feel bicycling on Fell Street has ...

the difficulty of making a right turn off of Fell Street while bicycling has...

the difficulty of navigating around turning motor vehicles has...

how often I ride a bicycle overall has....

$\begin{array}{c:c:c}\square_{1} & \square_{2} & \square_{3} \\ \square_{1} & \square_{2} & \square_{3} \\ \square_{1} & \square_{2} & \square_{3} \\ \square_{1} & \square_{2} & \square_{3} \\ \square_{1} & \square_{2} & \square_{3} \\ \square_{1} & \square_{2} & \square_{3} \\ \square_{1} & \square_{2} & \square_{3}\end{array}$

$\begin{array}{l:l}\square_{5} & \square_{9} \\ \square_{5} & \square_{9} \\ \square_{5} & \square_{9} \\ \square_{5} & \square_{9} \\ \square_{5} & \square_{9} \\ \square_{5} & \square_{9} \\ \square_{5} & \square_{9}\end{array}$

30. In the past $\mathbf{3 0}$ days, how many days have you bicycled for transportation (i.e. not just for recreation/ exercise)?

31. Did you fill out a separate online bicyclist survey about the Oak and Fell Street separated bikeways recently?
$\square_{1}$ Yes
$\square_{0}$ No 
32. Have you walked on this section of Oak and/ or Fell Streets since the separated bikeways were built?
Yes (Proceed to Question 33)
$\square_{0}$ No (Skip to Question 35)

33. Because of the Oak and Fell Street separated bikeways...

Decreased Decreased Not Increased Increased No a Lot Somewhat Changed Somewhat a Lot Opinion

drivers' speeds on Oak and Fell Street have generally ...

the number of bicyclists riding on the sidewalk has. .

my satisfaction with the walking environment on Oak and Fell Streets has. .

my sense of safety when crossing Oak and Fell Streets

has...

$\begin{array}{l:l:l}\square_{1} & \square_{2} & \square_{3} \\ \square_{1} & \square_{2} & \square_{3} \\ \square_{1} & \square_{2} & \square_{3} \\ \square_{1} & \square_{2} & \square_{3}\end{array}$

$\square_{4}$
$\square_{4}$
$\square_{4}$
$\square_{4}$

$\begin{array}{l:l}\square_{5} & \square_{9} \\ \square_{5} & \square_{9} \\ \square_{5} & \square_{9} \\ \square_{5} & \square_{9}\end{array}$

34. Do you ever walk or jog in the separated bikeway, rather than on the sidewalk?

$\square_{0}$ No

$\square_{1}$ Yes (Please explain why you choose the bikeway over the sidewalk in the space to the right) $\rightarrow$

\section{About Visiting Businesses on or near Oak and Fell Streets}

35. How many days per month do you spend money at businesses on or near Oak and Fell Streets? days

36. How did you travel to get there?

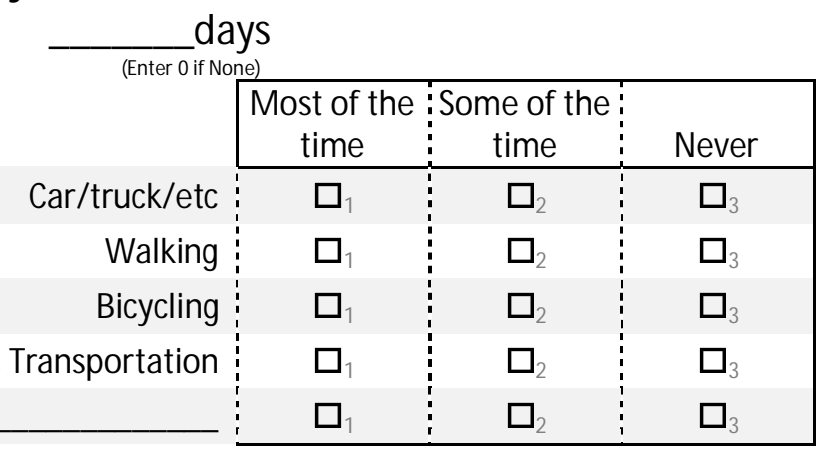

37. What types of businesses in the area do you patronize? (check all that apply)
$\square_{1}$ Retail Shops
$\square_{2}$ Banks
$\square_{3}$ Restaurants
$\square_{4}$ Bars
$\square_{5}$ Coffee Shop
$\square_{6}$ Other $->$

38. When you visit this area, how many businesses do you typically go to each time? (select the best response)

$\square_{1} 1$

$\square_{2} 2$

$\square$ 강

39. Since the separated bikeway was built, are you more or less likely to visit a business on or near Oak and Fell Streets?
$\square_{1}$ A lot less likely
$\square_{2}$ Somewhat less likely
$\square_{3}$ No change
$\square_{4}$ Somewhat more likely
$\square_{5}$ A lot more likely 
Overall Impressions of the Oak and Fell Street separated bikeways

40. What is the best thing about the separated bikeways?
41. What is the worst thing about the separated bikeways?

\section{About You}

We have a few questions about you so that we may understand the characteristics of our survey respondents. We will keep this information confidential and it is not linked to your name

42. Do you have a current: Yes No

$\begin{array}{rll}\text { Driver's license } & \square_{1} & \square_{0} \\ \text { Transit pass } & \square_{1} & \square_{0} \\ \text { Carshare Membership } & \square_{1} & \square_{0}\end{array}$

43. How many working motor vehicles does your household own or lease?

(Do not include motorhomes)

$$
\begin{aligned}
& \text { \#Vehicles } \\
& \text { (Enter ôif None-) }
\end{aligned}
$$

44. How many working adult bicycles does your household own?

$$
\text { \#Bicycles }
$$

45. INCLUDING YOURSELF, how many people live in your household?

$$
\begin{aligned}
& \text { \#Adults } \\
& \text { \#Children } \\
& \text { (Enter ōif Nonone) }
\end{aligned}
$$

46. How long have you lived at this home?

$$
\text { \#Years }
$$

47. Do you rent or own your home?
$\square_{1}$ Rent
$\square_{2}$ Own
$\square_{3}$ Other

48. Do you consider yourself:

(Select all that apply)

$\square$ American Indian or Alaska Native
$\square$ Asian
$\square$ Black or African American
$\square$ Hispanic or Latino/a
$\square$ White or Caucasian
$\square$ Other:

Thank you for taking our survey! If you would like to be entered in a random drawing for one of three \$100 Amazon gift cards please enter your name and contact information on the raffle slip you received with the survey.

54. Is there anything else you'd like to tell us? (Use the space below)
49. What is your age? __-_-_-_ years

50 . What is your gender?

51. What is your employment status? (Select all that apply)

$\square$ I work outside the home-> What is the zip code of

$\square$ I go to school outside the home-> What is the zip code of your school?

$\square$ I work from home

$\square$ Not employed at this time

52. What is your annual household income?

$\square_{1}$ Less than $\$ 25,000$

$\square, \$ 25,000$ to less than $\$ 50,000$

$\square$ $\$ 50,000$ to less than $\$ 75,000$

$\square$ 品 $\$ 75,000$ to less than $\$ 100,000$

$\square_{5} \$ 100,000$ to less than $\$ 200,000$

$\square_{6} \$ 200,000$ or more

$\square_{9}$ I prefer not to provide this information

53. What is the highest level of school you have completed?

$\square_{1}$ Some high school or less

$\square_{2}$ High school diploma or GED

$\square_{3}$ Some College

$\square_{4}$ Trade/Vocational School

$\square$ Associate Degree

$\square_{6}$ Four-year college degree or more

$\square_{9}$ Other your place of work? 


\section{RESIDENT: WASHINGTON DC - L STREET}




\section{Neighborhood Street Study}

\section{About How You Get Around}

\begin{tabular}{|c|c|c|c|c|c|c|}
\hline \multirow{2}{*}{$\begin{array}{l}\text { 1. For each mode of transportation, } \\
\text { please indicate your level of use: }\end{array}$} & \multicolumn{3}{|c|}{$\begin{array}{c}\text { Commute Trips } \\
\text { (to/from work or school) }\end{array}$} & \multicolumn{3}{|c|}{$\begin{array}{c}\text { Other Trips } \\
\text { (e.g. to the store, park, etc.) }\end{array}$} \\
\hline & Most Trips & Some Trips & No Trips & Most Trips: So & ne Trip & ps: No Trips \\
\hline Car/truck/motor vehicle (including carpool) & $\square_{1}$ & $\square_{2}$ & $\square_{3}$ & $\begin{array}{l:l}\square_{1} & \end{array}$ & $\square_{2}$ & $\square_{3}$ \\
\hline Walking & $\square_{1}$ & $\square_{2}$ & $\square_{3}$ & $\square_{1}$ & $\square_{2}$ & $\square_{3}$ \\
\hline Bicycling & $\square_{1}$ & $\square_{2}$ & $\square_{3}$ & $\square_{1}$ & $\square_{2}$ & $\square_{3}$ \\
\hline Public Transportation & $\square_{1}$ & $\square_{2}$ & $\square_{3}$ & $\square_{1}$ & $\square_{2}$ & $\square_{3}$ \\
\hline Other (please specify): & $\square_{1}$ & $\square_{2}$ & $\square_{3}$ & $\square_{1}$ & $\square_{2}$ & $\square_{3}$ \\
\hline \multicolumn{3}{|c|}{$\begin{array}{l}\text { 2. Compared to two years ago, are you taking more or } \\
\text { fewer trips by each mode of transportation? }\end{array}$} & More Trips & No Change & & Fewer Trips \\
\hline \multicolumn{3}{|c|}{ Car/truck/motor vehicle (including carpool) } & $\square_{1}$ & $\square_{2}$ & \multicolumn{2}{|c|}{$\square_{3}$} \\
\hline \multicolumn{3}{|c|}{ Walking } & $\square_{1}$ & $\square_{2}$ & \multicolumn{2}{|r|}{$\square_{3}$} \\
\hline \multicolumn{3}{|c|}{ Bicycling } & $\square_{1}$ & $\square_{2}$ & \multicolumn{2}{|r|}{$\square_{3}$} \\
\hline \multicolumn{3}{|c|}{ Public Transportation } & $\square_{1}$ & $\square_{2}$ & \multicolumn{2}{|r|}{$\square_{3}$} \\
\hline \multicolumn{3}{|c|}{ Other (please specify): } & $\square_{1}$ & $\square_{2}$ & \multicolumn{2}{|r|}{$\square_{3}$} \\
\hline
\end{tabular}

\section{About Your Neighborhood}

3. Over the past two years, changes to my neighborhood as a place for....

\begin{tabular}{|c|c|c|c|c|}
\hline $\begin{array}{c}\text { Very } \\
\text { Negative }\end{array}$ & $\begin{array}{l}\text { Somewhat } \\
\text { Negative }\end{array}$ & $\begin{array}{l}\text { No Impact/ } \\
\text { No Changes }\end{array}$ & $\begin{array}{c}\text { Somewhat } \\
\text { Positive }\end{array}$ & $\begin{array}{l}\text { Very } \\
\text { Positive }\end{array}$ \\
\hline$\square_{1}$ & $\square_{2}$ & $\square_{3}$ & $\square_{4}$ & $\square_{5}$ \\
\hline$\square_{1}$ & $\square_{2}$ & $\square_{3}$ & $\square_{4}$ & $\square_{5}$ \\
\hline$\square_{1}$ & $\square_{2}$ & $\square_{3}$ & $\square_{4}$ & $\square_{5}$ \\
\hline$\square_{1}$ & $\square_{2}$ & $\square_{3}$ & $\square$ & $\square_{5}$ \\
\hline
\end{tabular}

4. Overall, my level of satisfaction with transportation in my neighborhood is...
(1) Complete this paper survey and the enclosed raffle slip. Return in the postage-paid envelope;

OR

Complete the online survey and raffle entry at: http:// bit.ly/dc-streets To take the online survey, you will need to enter this code: D00013

Survey deadline extended to $06 / 18 / 2013$ 


\begin{tabular}{|c|c|c|c|c|c|}
\hline $\begin{array}{l}\text { 6. Please indicate whether you agree or disagree } \\
\text { with the following statements about bicycling: }\end{array}$ & $\begin{array}{l}\text { Strongly } \\
\text { Disagree }\end{array}$ & $\begin{array}{l}\text { Somewhat } \\
\text { Disagree }\end{array}$ & $\begin{array}{l}\text { Somewhat } \\
\text { Agree }\end{array}$ & $\begin{array}{c}\text { Strongly } \\
\text { Agree }\end{array}$ & $\begin{array}{c}\text { No } \\
\text { Opinion }\end{array}$ \\
\hline Bicycling in my city is a convenient way to get places. & $\mathbf{\square}_{1}$ & $\square_{2}$ & $\square_{3}$ & $\square_{4}$ & $\square_{9}$ \\
\hline Bicycling in my neighborhood is safe. & $\square_{1}$ & $\square_{2}$ & $\square_{3}$ & $\square_{4}$ & $\square_{9}$ \\
\hline $\begin{array}{l}\text { I am familiar with the bicycle lanes and other bicycle } \\
\text { facilities in my neighborhood. }\end{array}$ & $\square_{1}$ & $\square_{2}$ & $\square_{3}$ & $\square_{4}$ & $\square_{9}$ \\
\hline I would like to bicycle more often for transportation. & $\square_{1}$ & $\square_{2}$ & $\square_{3}$ & $\square_{4}$ & $\square_{9}$ \\
\hline $\begin{array}{l}\text { I would be more likely to ride a bicycle if motor vehicles } \\
\text { and bicycles were physically separated by a barrier. }\end{array}$ & $\square_{1}$ & $\square_{2}$ & $\square_{3}$ & $\square_{4}$ & $\square_{9}$ \\
\hline $\begin{array}{l}\text { Facilities that encourage bicycling for transportation are } \\
\text { a good way to improve public health. }\end{array}$ & $\square_{1}$ & $\square_{2}$ & $\square_{3}$ & $\square_{4}$ & $\square_{9}$ \\
\hline
\end{tabular}

\section{Whether or not you currently ride a bicycle, please consider how comfortable you would be riding a bicycle in each place:}

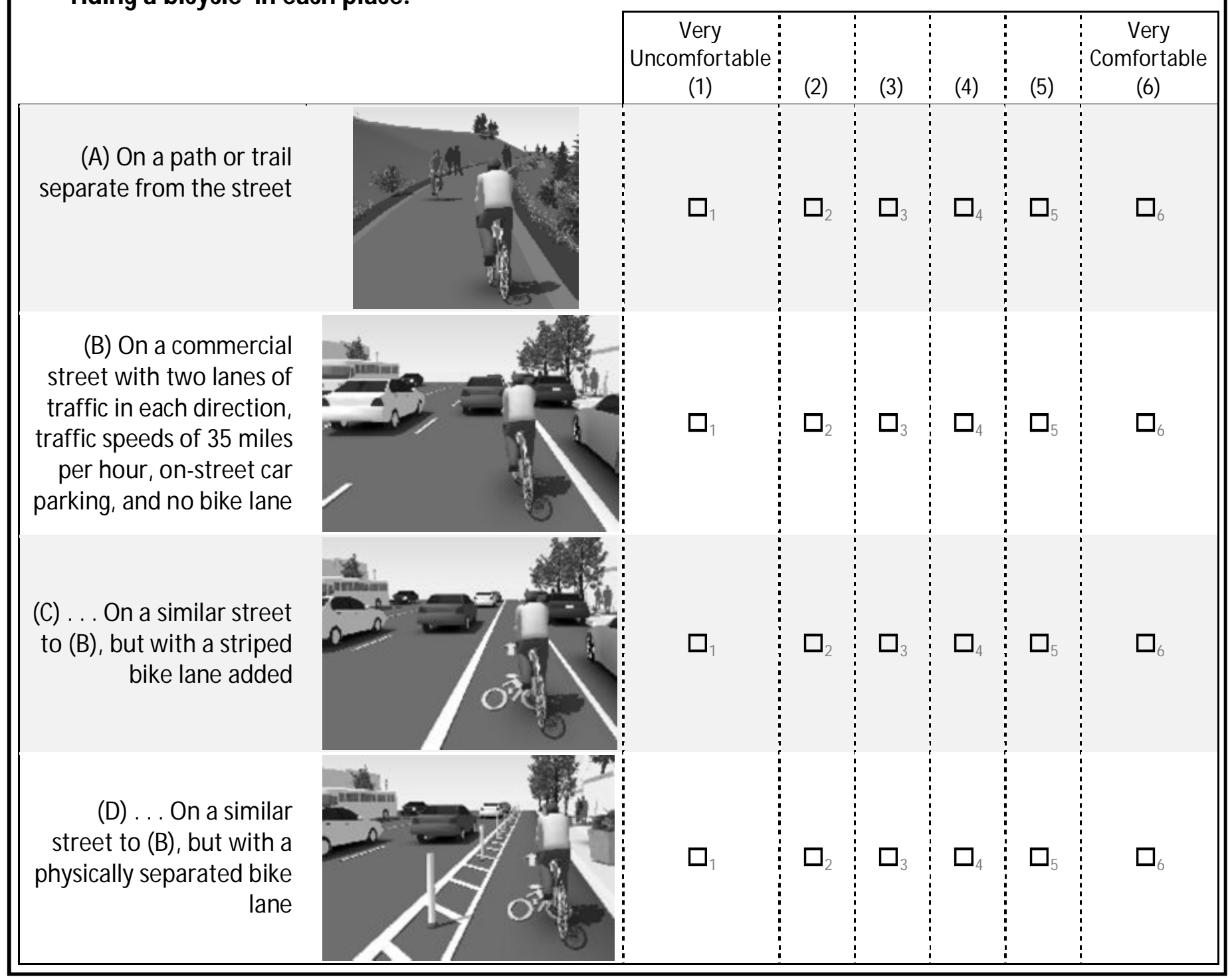




\section{$\underline{\text { LStreet Cycle Track }}$}

A separated eastbound bikeway, also known as a "cycle track," was recently built on L Street NW from New Hampshire Avenue to 12th Street. In most places, a standard traffic lane was converted to a bicycle lane with a painted "buffer" area and plastic "flexposts" separating the bike lane from the motor vehicle traffic lanes (see pictures below). The questions on the following pages are related to this cycle track.

\section{LStreet before and now:}

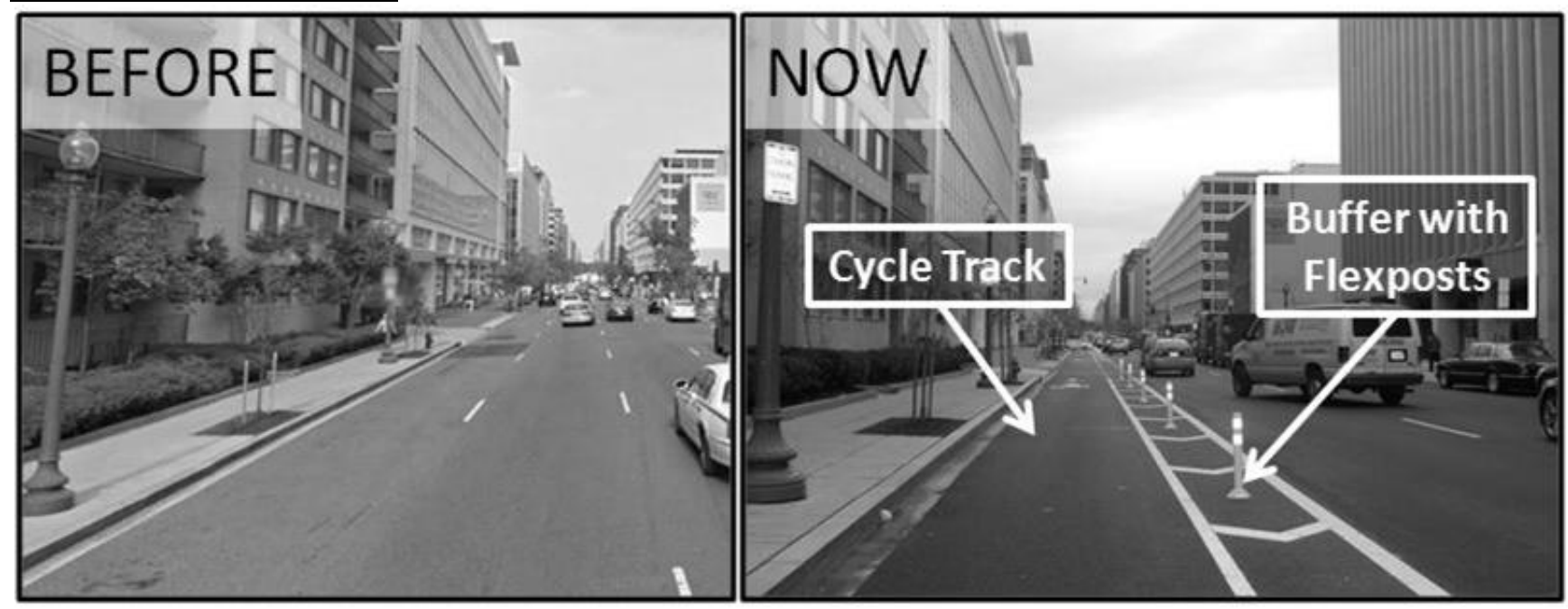

\section{Extent of L Street cycle track:}

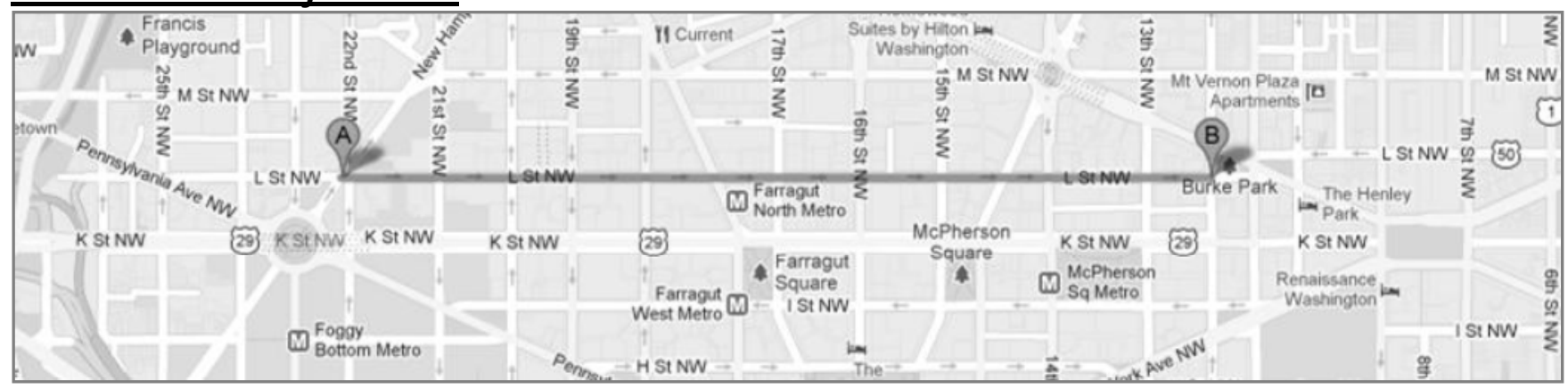

\section{About the L Street Cycle Track}

8. Because of the LStreet cycle track ... .

\begin{tabular}{|c|c|c|c|c|c|c|}
\hline ( & $\begin{array}{c}\text { Decreased } \\
\text { a Lot }\end{array}$ & $\begin{array}{l}\text { Decreased } \\
\text { Somewhat }\end{array}$ & $\begin{array}{c}\text { Not } \\
\text { Changed }\end{array}$ & $\begin{array}{l}\text { Increased } \\
\text { Somewhat }\end{array}$ & \begin{tabular}{|c} 
Increased \\
a Lot
\end{tabular} & $\begin{array}{c}\text { No } \\
\text { Opinion }\end{array}$ \\
\hline $\begin{array}{l}\text { the desirability of living in my } \\
\text { neighborhood has... }\end{array}$ & $\square_{1}$ & $\square_{2}$ & $\square_{3}$ & $\square_{4}$ & $\square_{5}$ & $\square_{9}$ \\
\hline the aesthetic appeal of $L$ Street has... & $\square_{1}$ & $\square_{2}$ & $\square_{3}$ & $\square_{4}$ & $\square_{5}$ & $\square_{9}$ \\
\hline the safety of DRIVING on L Street has ... & $\square_{1}$ & $\square_{2}$ & $\square_{3}$ & $\square_{4}$ & $\square_{5}$ & $\square_{9}$ \\
\hline the safety of BICYCLING on L Street has. . . & $\square_{1}$ & $\square_{2}$ & $\square_{3}$ & $\square_{4}$ & $\square_{5}$ & $\square_{9}$ \\
\hline the safety of WALKING on L Street has... & $\square_{1}$ & $\square_{2}$ & $\square_{3}$ & $\square_{4}$ & $\square_{5}$ & $\square_{9}$ \\
\hline $\begin{array}{r}\text { the number of people I see riding bikes } \\
\text { on L Street has ... }\end{array}$ & $\square_{1}$ & $\square_{2}$ & $\square_{3}$ & $\square_{4}$ & $\square_{5}$ & $\square_{9}$ \\
\hline how well the road works for all people has... & $\square_{1}$ & $\square_{2}$ & $\square_{3}$ & $\square_{4}$ & $\square_{5}$ & $\square_{9}$ \\
\hline
\end{tabular}


About the L Street Cycle Track, cont.

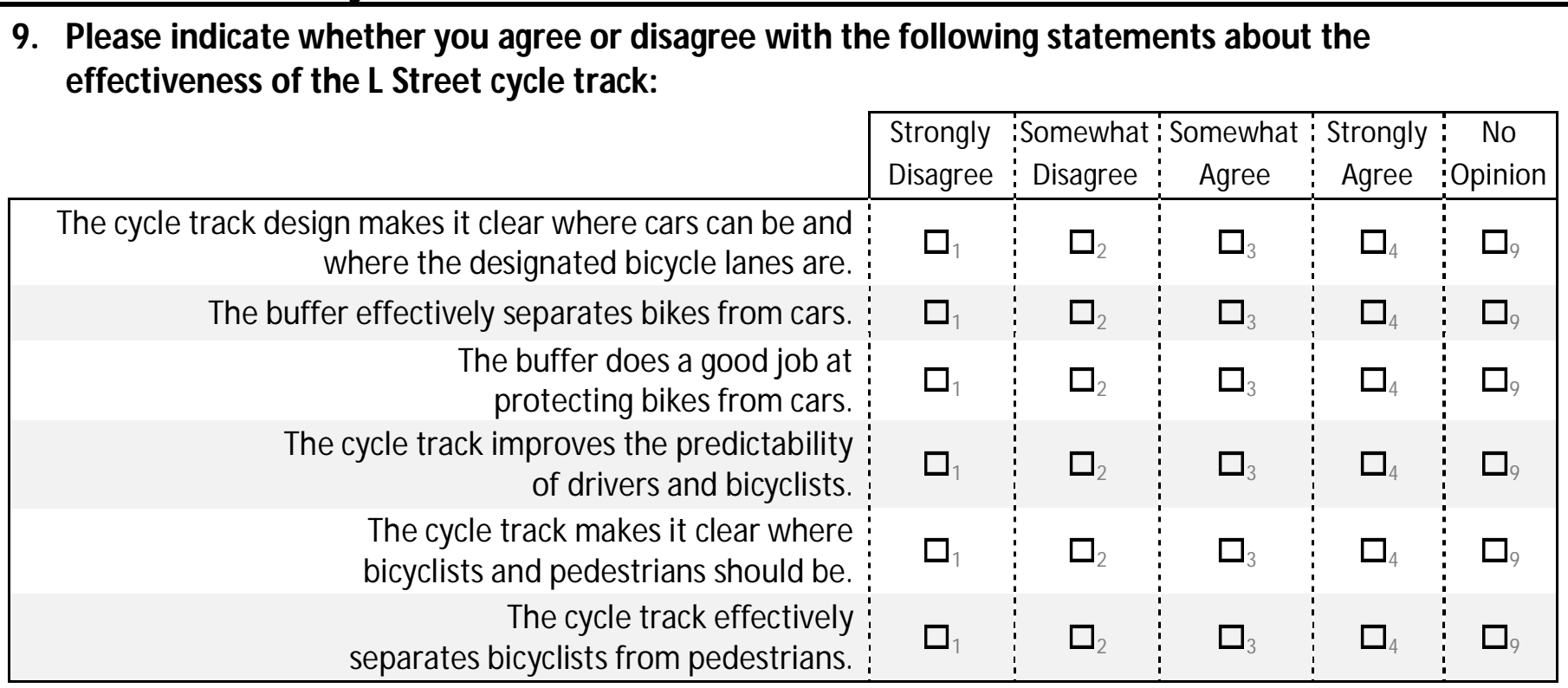

10. Over time, my opinion of the L Street cycle track has become:

\begin{tabular}{|c|c|c|c|c|}
\hline $\begin{array}{c}\text { A Lot More } \\
\text { Negative }\end{array}$ & $\begin{array}{c}\text { Somewhat } \\
\text { More Negative }\end{array}$ & $\begin{array}{c}\text { Somewhat } \\
\text { Nonange }\end{array}$ & $\begin{array}{c}\text { A Lot M ore Positive } \\
\text { Positive }\end{array}$ \\
\hline$\square_{1}$ & $\square_{2}$ & $\square_{3}$ & $\square_{4}$ & $\square_{5}$ \\
\hline
\end{tabular}

11. Please indicate whether you agree or disagree with the following statements about cycle tracks.

I would support the District in building more cycle : tracks at other locations.

Overall, I support separating bikes from cars.

\begin{tabular}{|c|c:c:c:c|}
\hline $\begin{array}{c}\text { Strongly } \\
\text { Disagree }\end{array}$ & $\begin{array}{c}\text { Somewhat } \\
\text { Disagree }\end{array}$ & $\begin{array}{c}\text { Somewhat } \\
\text { Agree }\end{array}$ & $\begin{array}{c}\text { Strongly } \\
\text { Agree }\end{array}$ & $\begin{array}{c}\text { No } \\
\text { Opinion }\end{array}$ \\
\hline$\square_{1}$ & $\square_{2}$ & $\square_{3}$ & $\square_{4}$ & $\square_{9}$ \\
$\square_{1}$ & $\square_{2}$ & $\square_{3}$ & $\square_{4}$ & $\square_{9}$ \\
\hline
\end{tabular}

\section{About Driving on L Street}

12. Have you driven a motor vehicle on this section of $L$ Street since the cycle track was built?

$\square_{1}$ Yes (Proceed to Question 13) $\quad \square_{0}$ No (Skip to Question 18)

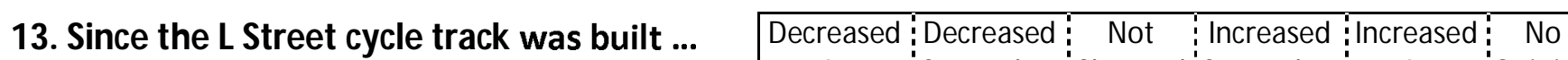

\begin{tabular}{|c|c|c|c|c|c|c|}
\hline & a Lot & Somewhat & Changed & Somewhat & a Lot & Opinion \\
\hline $\begin{array}{r}\text { the number of bicyclists riding in the same lanes } \\
\text { with cars on L Street has... }\end{array}$ & $\square_{1}$ & $\square_{2}$ & $\square_{3}$ & $\square_{4}$ & $\square_{5}$ & $\square_{9}$ \\
\hline how safe and predictable bicyclists are acting has ... & $\square_{1}$ & $\square_{2}$ & $\square_{3}$ & $\square_{4}$ & $\square_{5}$ & $\square_{9}$ \\
\hline how safe and predictable drivers are acting has ... & $\square_{1}$ & $\square_{2}$ & $\square_{3}$ & $\square_{4}$ & $\square_{5}$ & $\square_{9}$ \\
\hline $\begin{array}{r}\text { the amount of time it takes me to drive } \\
\text { on } L \text { Street has... }\end{array}$ & $\square_{1}$ & $\square_{2}$ & $\square_{3}$ & $\square_{4}$ & $\square_{5}$ & $\square_{9}$ \\
\hline
\end{tabular}


About Driving on L Street, cont.

\begin{tabular}{|c|c|c|c|c|c|}
\hline 14. The impact of the L Street cycle track on . . . & $\begin{array}{c}\text { Very } \\
\text { Negative }\end{array}$ & $\begin{array}{l}\text { Somewhat } \\
\text { Negative }\end{array}$ & $\begin{array}{l}\text { No Impact/ } \\
\text { Neutral }\end{array}$ & $\begin{array}{l}\text { Somewhat } \\
\text { Positive }\end{array}$ & $\begin{array}{l}\text { Very } \\
\text { Positive }\end{array}$ \\
\hline traffic congestion has been . . & $\square_{1}$ & $\square_{2}$ & $\square_{3}$ & $\square_{4}$ & $\square_{5}$ \\
\hline $\begin{array}{r}\text { my ability to turn off of } L \text { Street at signalized } \\
\text { intersections has been ... }\end{array}$ & $\square_{1}$ & $\square_{2}$ & $\square_{3}$ & $\square_{4}$ & $\square_{5}$ \\
\hline $\begin{array}{r}\text { my ability to turn off of } L \text { Street into alleys, driveways, } \\
\text { and parking garages has been ... }\end{array}$ & $\square_{1}$ & $\square_{2}$ & $\square_{3}$ & $\square_{4}$ & $\square_{5}$ \\
\hline $\begin{array}{r}\text { my ability to pull onto L Street from alleys, driveways, } \\
\text { and parking garages has been ... }\end{array}$ & $\square_{1}$ & $\square_{2}$ & $\square_{3}$ & $\square_{4}$ & $\square_{5}$ \\
\hline my ability to find a parking spot on L Street has been ... & $\square_{1}$ & $\square_{2}$ & $\square_{3}$ & $\square_{4}$ & $\square_{5}$ \\
\hline how stressful it is to park a car on L Street has been ... & $\square_{1}$ & $\square_{2}$ & $\square_{3}$ & $\square_{4}$ & $\square_{5}$ \\
\hline
\end{tabular}

15. At many intersections on L Street, drivers must merge across the bike lane through a "mixing zone," and into a left-turn lane in order to complete a left turn (see picture).

Please indicate whether you agree or disagree with the following statements about the mixing zone:

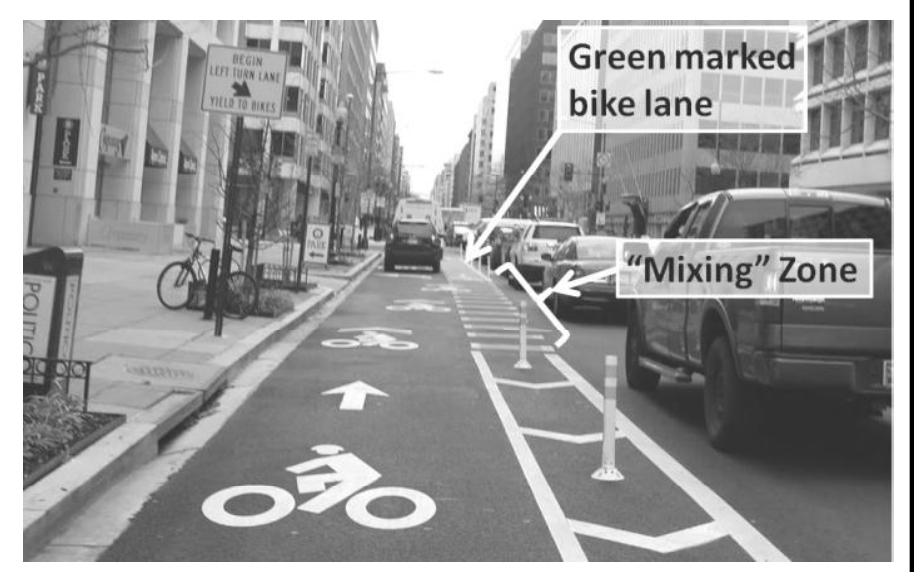

\begin{tabular}{|c|c|c|c|c|c|}
\hline & \multirow{2}{*}{$\begin{array}{l}\text { Strongly } \\
\text { Disagree }\end{array}$} & \multicolumn{2}{|c|}{ Somewhat:Somewhat } & \multirow{2}{*}{$\begin{array}{c}\text { Strongly } \\
\text { Agree }\end{array}$} & \multirow{2}{*}{$\begin{array}{c}\text { No } \\
\text { Opinior }\end{array}$} \\
\hline & & Disagree & Agree & & \\
\hline $\begin{array}{r}\text { When I want to make a left turn, I am able to } \\
\text { adequately see if there are any approaching bicyclists in } \\
\text { the cycle track. }\end{array}$ & $\square_{1}$ & $\square_{2}$ & $\square_{3}$ & $\square_{4}$ & $\square_{9}$ \\
\hline $\begin{array}{r}\text { The designated mixing zone for left-turning vehicles } \\
\text { provides me adequate time/space to move into } \\
\text { the left-turn lane. }\end{array}$ & $\square_{1}$ & $\square_{2}$ & $\square_{3}$ & $\square_{4}$ & $\square_{9}$ \\
\hline $\begin{array}{l}\text { When attempting to turn left off of } \mathrm{L} \text { Street, I am } \\
\text { sometimes unable to move into the designated left-turn } \\
\text { lane, and forced to turn from another lane. }\end{array}$ & $\square_{1}$ & $\square_{2}$ & $\square_{3}$ & $\square_{4}$ & $\square_{9}$ \\
\hline
\end{tabular}

16. How often do you encounter delivery vehicles parked in the mixing zone or left-turn lane?
$\square_{1}$ Never
Rarely
Sometimes
Usually

17. Do you avoid driving on L Street because of the cycle track?
$\square_{1}$ Yes
$\square_{0}$ No 
About Bicycling on the L Street Cycle Track

18. Have you bicycled on this section of LStreet since the cycle track was built?

$\square_{1}$ Yes (Proceed to Question 19) $\square_{0}$ No (Skip to Question 23)

19. How comfortable do you feel when bicycling in the L Street cycle track?

\begin{tabular}{|c|c|c|c|c|c|}
\hline $\begin{array}{c}\text { Very } \\
\text { Uncomfortable } \\
(1)\end{array}$ & (2) & (3) & (4) & (5) & $\begin{array}{c}\text { Very } \\
\text { Comfortable } \\
\text { (6) }\end{array}$ \\
\hline$\square_{1}$ & $\square_{2}$ & $\square_{3}$ & 口 & $\square_{5}$ & $\square_{6}$ \\
\hline
\end{tabular}

20. Because of the LStreet cycle track ...

\begin{tabular}{|c|c|c|c|c|c|c|}
\hline & $\begin{array}{c}\text { Decreased } \\
\text { a Lot }\end{array}$ & $\begin{array}{l}\text { Decreased } \\
\text { Somewhat }\end{array}$ & $\begin{array}{c}\text { Not } \\
\text { Changed }\end{array}$ & $\begin{array}{l}\text { Increased } \\
\text { Somewhat }\end{array}$ & $\begin{array}{c}\text { Increased } \\
\text { a Lot }\end{array}$ & \begin{tabular}{|c|} 
No \\
Opinion
\end{tabular} \\
\hline $\begin{array}{l}\text { the likelihood that I will choose to bicycle on } \\
\text { L Street as opposed to other streets has... }\end{array}$ & $\square_{1}$ & $\square_{2}$ & $\square_{3}$ & $\square_{4}$ & $\square_{5}$ & $\square_{9}$ \\
\hline the time it takes me to bicycle on L Street has.. & $\square_{1}$ & $\square_{2}$ & $\square_{3}$ & $\square_{4}$ & $\square_{5}$ & $\square_{9}$ \\
\hline $\begin{array}{r}\text { the usefulness of } L \text { Street for getting places } \\
\qquad \text { I want to go has... }\end{array}$ & $\square_{1}$ & $\square_{2}$ & $\square_{3}$ & $\square_{4}$ & $\square_{5}$ & $\square_{9}$ \\
\hline $\begin{array}{r}\text { how often I stop at shops and businesses } \\
\text { on LStreet has ... }\end{array}$ & $\square_{1}$ & $\square_{2}$ & $\square_{3}$ & $\square_{4}$ & $\square_{5}$ & $\square_{9}$ \\
\hline drivers' awareness of bicyclists on L Street has... & $\square_{1}$ & $\square_{2}$ & $\square_{3}$ & $\square_{4}$ & $\square_{5}$ & $\square_{9}$ \\
\hline drivers' speeds on L Street have . & $\square_{1}$ & $\square_{2}$ & $\square_{3}$ & $\square_{4}$ & $\square_{5}$ & $\square_{9}$ \\
\hline $\begin{array}{r}\text { how comfortable I feel when bicycling } \\
\text { on LStreet has ... }\end{array}$ & $\square_{1}$ & $\square_{2}$ & $\square_{3}$ & $\square_{4}$ & $\square_{5}$ & $\square_{9}$ \\
\hline how often I ride a bicycle overall has... & $\square_{1}$ & $\square_{2}$ & $\square_{3}$ & $\square_{4}$ & $\square_{5}$ & $\square_{9}$ \\
\hline $\begin{array}{l}\text { the difficulty of making a right-turn off of } L \text { Street } \\
\qquad \text { while bicycling has... }\end{array}$ & $\square_{1}$ & $\square_{2}$ & $\square_{3}$ & $\square_{4}$ & $\square_{5}$ & $\square_{9}$ \\
\hline $\begin{array}{l}\text { the difficulty of navigating around } \\
\text { turning motor vehicles has... }\end{array}$ & $\square_{1}$ & $\square_{2}$ & $\square_{3}$ & $\square_{4}$ & $\square_{5}$ & $\square_{9}$ \\
\hline $\begin{array}{r}\text { the difficulty of navigating around } \\
\text { pedestrians has... }\end{array}$ & $\square_{1}$ & $\square_{2}$ & $\square_{3}$ & $\square_{4}$ & $\square_{5}$ & $\square_{9}$ \\
\hline
\end{tabular}

21. In the past 30 days, how many days have you bicycled for transportation (i.e. not just for recreation/ exercise)? days (Enter oif None)

22. Did you fill out a separate online bicyclist survey about the $L$ Street cycle track from us recently?
$\square_{1}$ Yes
No 
About Walking on L Street

23. Have you walked on this section of $L$ Street since the cycle track was built?

$\square_{1}$ Yes (Proceed to Question 24) $\quad \square_{0}$ No (Skip to Question 26)

\begin{tabular}{|c|c|c|c|c|c|c|}
\hline 24. Because of the L Street cycle track . . . & $\begin{array}{c}\text { Decreased } \\
\text { a Lot }\end{array}$ & $\begin{array}{l}\text { Decreased } \\
\text { Somewhat }\end{array}$ & $\begin{array}{c}\text { Not } \\
\text { Changed }\end{array}$ & $\begin{array}{l}\text { Increased } \\
\text { Somewhat }\end{array}$ & $\begin{array}{c}\text { Increased } \\
\text { a Lot }\end{array}$ & $\begin{array}{c}\text { No } \\
\text { Opinion }\end{array}$ \\
\hline drivers' speeds on L Street have generally. & $\square_{1}$ & $\square_{2}$ & $\square_{3}$ & $\square_{4}$ & $\square_{5}$ & $\square_{9}$ \\
\hline $\begin{array}{r}\text { the number of bic } \\
\text { on the side }\end{array}$ & $\square_{1}$ & $\square_{2}$ & $\square_{3}$ & $\square_{4}$ & $\square_{5}$ & $\square_{9}$ \\
\hline $\begin{array}{r}\text { my satisfaction with the walking environment } \\
\text { on LStreet has... }\end{array}$ & $\square_{1}$ & $\square_{2}$ & $\square_{3}$ & $\square_{4}$ & $\square_{5}$ & $\square_{9}$ \\
\hline ny sense of safety when crossing L Street has & $\square_{1}$ & $\square_{2}$ & $\square_{3}$ & $\square_{4}$ & $\square_{5}$ & $\square_{9}$ \\
\hline
\end{tabular}

25. Do you ever walk or jog in the cycle track, rather than on the sidewalk?

$\square$ No

$\square_{1}$ Yes (Please explain why you choose the cycle track over the sidewalk in the space to the right) $\rightarrow$

\section{About Visiting Businesses on L Street}

26. How many days per month do you spend money at businesses on or near L Street?

27. How did you travel to get there?

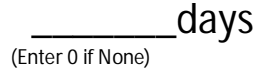

\begin{tabular}{r|c:c:c} 
& time & time & Never \\
Car/truck/etc & $\square_{1}$ & $\square_{2}$ & $\square_{3}$ \\
Walking & $\square_{1}$ & $\square_{2}$ & $\square_{3}$ \\
Bicycling & $\square_{1}$ & $\square_{2}$ & $\square_{3}$ \\
Public Transportation & $\square_{1}$ & $\square_{2}$ & $\square_{3}$ \\
Other (please specify): & $\square_{1}$ & $\square_{2}$ & $\square_{3}$ \\
\hline
\end{tabular}

28. What types of businesses in the area do you patronize? (check all that apply)
$\square_{1}$ Grocery Store
$\square_{2}$ Retail Shops
$\square_{3}$ Restaurants
$\square_{5}$ Banks
$\square_{6}$ Post Office
$\square_{7}$ Coffee Shop
$\square_{8}$ Other ->

29. When you visit this area, how many businesses do you typically go to each time? (select the best response)

$\square_{1} 1$

$\square_{2} 2$

$\square_{3} 3$ or more

30. Since the cycle track was built, are you more or less likely to visit a business on L Street? $\square_{1}$ A lot less likely

$\square_{2}$ Somewhat less likely

$\square_{3}$ No change

$\square_{4}$ Somewhat more likely

$\square_{5}$ A lot more likely 


\section{About You}

We have a few questions about you so that we may understand the characteristics of our survey respondents. We will keep this information confidential and it is not linked to your name
33. Do you have a current:
Driver's license
Transit pass
Bikeshare M embership
Carshare M embership
Yes No
$\square_{1}$
$\square_{1}$
$\square_{1}$
$\square_{1}$

34. How many working motor vehicles does your household own or lease?

(Do not include motorhomes)

$$
\begin{aligned}
& \text { \# Vehicles } \\
& \text { (Enter } 0 \text { if } \overline{\text { None }} \text { ) }
\end{aligned}
$$

35. How many working adult bicycles does your household own?

$$
\begin{aligned}
& \text { \#Bicycles } \\
& \text { (Enter } 0 \text { if } \\
&
\end{aligned}
$$

36. INCLUDING YOURSELF, how many people live in your household?

$$
\begin{aligned}
& \text { \#Adults } \\
& \text { \#Children } \\
& \text { (Enter } 0 \text { if }- \text { None) }
\end{aligned}
$$

37. How long have you lived at this home?

$$
\text { \#Years }
$$

38. Do you rent or own your home?
$\square_{1}$ Rent
$\square_{2}$ Own
$\square$ Other

39. Do you consider yourself:

(Select all that apply)

$\square$ American Indian or Alaska Native
$\square$ Asian
$\square$ Black or African American
$\square$ Hispanic or Latino/a
$\square$ White or Caucasian
$\square$ Other:

Thank you for taking our survey! If you would like to be entered in a random drawing for one of three \$100 Amazon gift cards please enter your name and contact information on the raffle slip you received with the survey.

45. Is there anything else you'd like to tell us? (Use the space below) 


\section{BICYCLIST: AUSTIN - BARTON SPRINGS}




\section{Neighborhood Street Study}

Default Question Block

\section{Dear Bicyclist,}

My name is Chris Monsere and I am a faculty member at Portland State University in the Department of Civil and Environmental Engineering. I am the principal investigator on a project researching separated bicycle lanes in six cities across the country.

Hearing from bicyclists like yourself is a very important part of this study. We are only sampling a select number of bicyclists near the Bluebonnet Lane cycle track. Therefore, every response is very important and we hope you will participate. We will share our findings with the City of Austin and hope that the results will help in future plans for improving bicycling in cities around the United States.

The survey, which starts on the next page, should take about 10-20 minutes. Your participation in the study is voluntary and you may stop taking the survey at any time with no consequences.

The postcard that you received has a unique number that only identifies where and when we handed you the postcard. We will protect the confidentiality of your individual survey responses. None of your responses will be linked to your name or other identifying personal information.

To say thank you, all people who complete their survey by July 5, 2013 will be entered into a drawing for one of three $\$ 100$ Amazon.com gift cards. You will be asked to enter your name and a way to contact you at the end of the survey - this information is voluntary and will not be connected to your survey data.

If you have concerns or problems about your participation in this study or your rights as a research subject, please contact the Human Subjects Research Review Committee, Office of Research and Strategic Partnerships, Market Center Building Suite 620, Portland State University, (877480-4400). If you have questions about the study itse If, please contact our research team directly at streets@pdx.edu or 503-725-2875.

Sincerely,

Christopher M. Monsere, Ph.D., P.E., Associate Professor \& Associate Chair Civil and Environmental Engineering Portland State University

Do you agree to participate in this survey?

$$
\begin{aligned}
& \text { No } \\
& \text { Yes }
\end{aligned}
$$

When you received the postcard for this survey, where were you ...

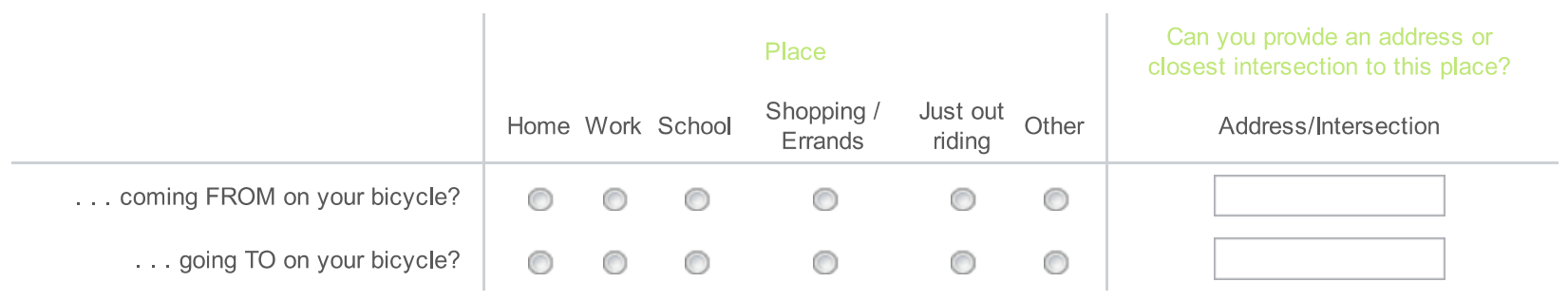

If you selected other, please explain: 


\section{Bluebonnet Lane Cycle Track}

A separated two-way bikeway, also known as a "cycle track," was recently built on Bluebonnet Lane from Rabb Glenn Street to Rabb Road. To do this, the parking/bike lane that existed before on the west side of the road was converted to a two-way bicycle lane with a painted "buffer" area and plastic "flexposts" separating the cycle track from the standard traffic lane (see pictures below). The questions on the following pages are related to this cycle track.

Bluebonnet Lane before and now:
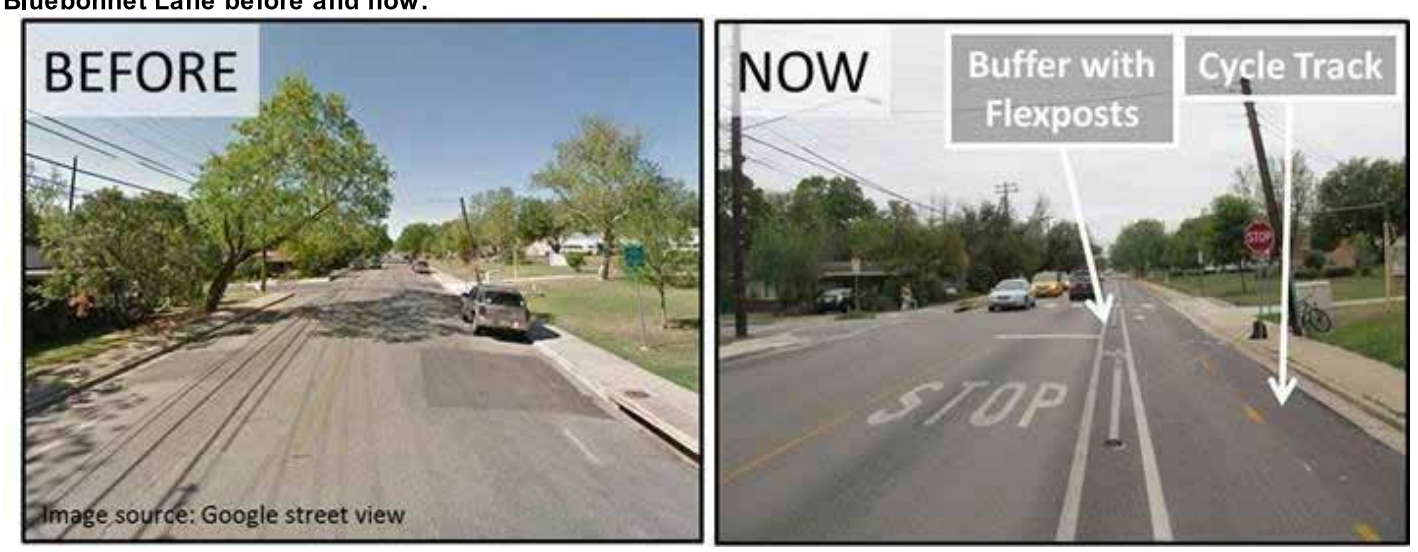

Extent of Bluebonnet Lane cycle track:

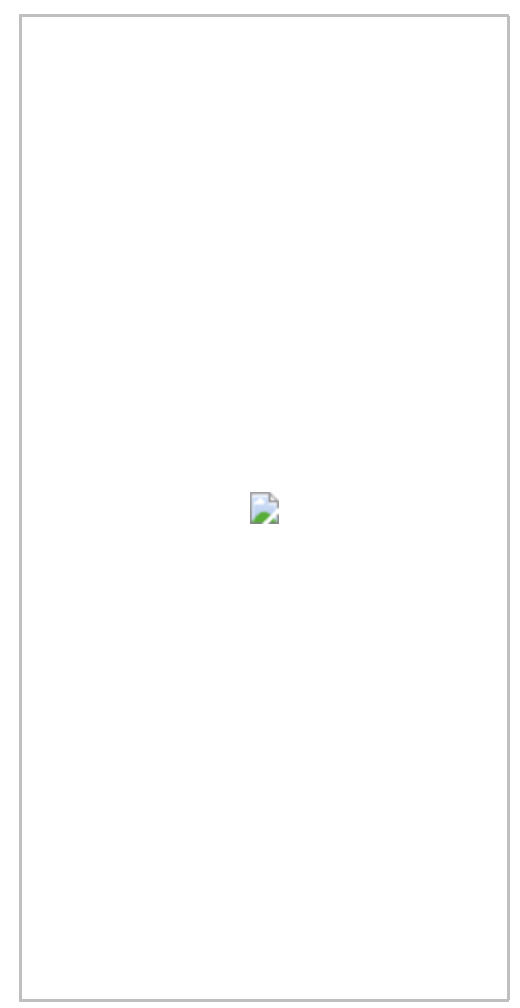

How often do you ride a bicycle on this part of Bluebonnet Lane?
This was my first time
Less than once a month
A few times a month
A few times a week
Daily 
How comfortable do you feel when bicycling in the Bluebonnet Lane cycle track in the lane closest to the sidewalk (i.e. southbound)?
(1) Very Uncomfortable
(2)
(3)
(4)
(5)
(6) Very Comfortable

How comfortable do you feel when bicycling in the Bluebonnet Lane cycle track in the lane close st to the standard traffic lanes and the plastic flexposts (i.e. northbound)?
(1) Very Uncomfortable
(2)
(3)
(4)
(5)
(6) Very Comfortable

Since the Bluebonnet Lane cycle track was built, do you travel on this route:
Less frequently
More frequently
About the same
This is my first time on this route

When bicycling on Bluebonnet Lane, how often do you ride on the following:

\begin{tabular}{l|cccc} 
& Always & Most of the time & Some of the time & Never \\
\hline Cycle track & 0 & 0 & 0 & 0 \\
Standard traffic lanes & 0 & 0 & 0 & 0 \\
Sidewalk & 0 & 0 & 0
\end{tabular}

Consider the trip you were making when you were handed the postcard. Before the Bluebonnet Lane cycle track was built, how would you have made this trip?
By bicycle, using this same route
By bicycle, using another route - (Please specify which route):
By foot
By public transportation
By car
By taxi
I would NOT have taken this trip
Other (please specify below) 


\section{About Changes due to the Cycle Track}

\section{Because of the Bluebonnet Lane cycle track...}

\begin{tabular}{|c|c|c|c|c|c|c|}
\hline & $\begin{array}{l}\text { Decreased } \\
\quad \text { a Lot }\end{array}$ & $\begin{array}{l}\text { Decreased } \\
\text { Somewhat }\end{array}$ & $\begin{array}{c}\text { Not } \\
\text { Changed }\end{array}$ & $\begin{array}{l}\text { Increased } \\
\text { Somewhat }\end{array}$ & $\begin{array}{l}\text { Increased } \\
\quad \text { a Lot }\end{array}$ & $\begin{array}{l}\text { No } \\
\text { Opinion }\end{array}$ \\
\hline I feel the safety of bicycling on Bluebonnet Lane has... & O & O & O & ○ & O & O \\
\hline the time it takes me to bicycle on Bluebonnet Lane has... & O & O & ○ & O & O & ○ \\
\hline $\begin{array}{r}\text { the usefulness of Bluebonnet Lane for getting to places I want to go } \\
\text { has ... }\end{array}$ & ○ & O & ○ & O & O & 0 \\
\hline how comfortable I feel when bicycling on Bluebonnet Lane has . . . & O & O & O & ○ & O & ○ \\
\hline drivers' awareness of people biking on Bluebonnet Lane has ... & ○ & O & ○ & ○ & 0 & O \\
\hline drivers' speeds on Bluebonnet Lane have... & O & O & O & 0 & O & O \\
\hline how often I ride a bicycle overall has ... & O & O & O & O & O & O \\
\hline the difficulty of navigating around pedestrians has ... & 0 & 0 & 0 & 0 & 0 & ○ \\
\hline the difficulty of turning across Bluebonnet Lane while bicycling has. & 0 & 0 & O & O & O & O \\
\hline how comfortable I feel bicycling with my kids has... & O & O & O & ○ & 0 & O \\
\hline how safe I feel bicycling with my kids has ... & O & O & 0 & ○ & O & 0 \\
\hline
\end{tabular}

\section{Your Opinions about the Cycle Track}

Please indicate if you agree or disagree with the following statements about the cycle track:

I would go out of my way to ride on Bluebonnet Lane compared to other
Taking Bluebonnet Lane is the most direct bicycle route to my destination.
The buffer and flexposts between the traffic lanes and the cycle track make
me feel safe.
Leaves and debris in the cycle track are worse than other places I ride.
The cycle track is wide enough for me to ride comfortably.
The cycle track is wide enough for one bicyclist to pass another.
The cycle track is wide enough for two people to comfortably ride side-by-
If $\mathrm{I}$ am bicycle.
The cycle track is safer than other bike lanes in Austin.
The cycle track is wide enough for me to be comfortable when passing by a
bicyclist going in the opposite direction

Please indicate if you disagree or agree with the following statements about the effectiveness of the Bluebonnet Lane cycle track:

\begin{tabular}{|c|c|c|c|c|c|}
\hline & $\begin{array}{l}\text { Strongly } \\
\text { Disagree }\end{array}$ & $\begin{array}{l}\text { Somewhat } \\
\text { Disagree }\end{array}$ & $\begin{array}{l}\text { Somewhat } \\
\text { Agree }\end{array}$ & $\begin{array}{l}\text { Strongly } \\
\text { Agree }\end{array}$ & No Opinion \\
\hline $\begin{array}{l}\text { The cycle track makes it clear where cars can be and where } \\
\text { the designated bicycle lanes are. }\end{array}$ & ○ & ○ & O & ○ & ○ \\
\hline $\begin{array}{c}\text { The intersection signs and street markings make it clear who } \\
\text { has the right-of-way at intersections. }\end{array}$ & ○ & O & O & ○ & ○ \\
\hline The buffer and flexposts effectively separate bikes from cars. & 0 & O & O & O & O \\
\hline $\begin{array}{r}\text { The buffer and flexposts do a good job at protecting bikes from } \\
\text { cars. }\end{array}$ & ○ & ○ & O & ○ & O \\
\hline The cycle track makes drivers and bicyclists more predictable. & ○ & ○ & O & ○ & O \\
\hline $\begin{array}{r}\text { The cycle track makes it clear where bicyclists and pedestrians } \\
\text { should be. }\end{array}$ & O & O & O & O & O \\
\hline
\end{tabular}




\section{About What You Encounter in the Cycle Track}

Please indicate how often you have observed the following on your rides in the Bluebonnet Lane cycle track, AND whether you think this is a problem that needs to be addressed

\begin{tabular}{|c|c|c|c|c|c|c|c|}
\hline & \multicolumn{4}{|c|}{$\begin{array}{l}\text { How often do you encounter the following } \\
\qquad \text { IN the CYCLE TRACK? }\end{array}$} & \multicolumn{3}{|c|}{ How much of a problem is this? } \\
\hline & Never & Rarely & Sometimes & Often & $\begin{array}{l}\text { Not a } \\
\text { problem }\end{array}$ & $\begin{array}{l}\text { Minor } \\
\text { problem }\end{array}$ & $\begin{array}{l}\text { Major } \\
\text { problem }\end{array}$ \\
\hline Cars parking & 0 & O & 0 & O & O & ○ & O \\
\hline $\begin{array}{l}\text { Cars loading or } \\
\text { unloading passengers }\end{array}$ & O & O & ○ & O & O & 0 & ○ \\
\hline $\begin{array}{l}\text { Buses loading or } \\
\text { unloading passengers }\end{array}$ & O & O & 0 & O & O & O & O \\
\hline Taxis & 0 & 0 & 0 & 0 & ○ & 0 & 0 \\
\hline Cars/trucks driving & ○ & O & O & O & ○ & O & ○ \\
\hline $\begin{array}{c}\text { Cars/trucks waiting to } \\
\text { make turns OFF of } \\
\text { Bluebonnet Lane }\end{array}$ & O & O & O & O & O & O & O \\
\hline $\begin{array}{r}\text { Cars/trucks waiting to } \\
\text { pull ONTO } \\
\text { Bluebonnet Lane }\end{array}$ & 0 & 0 & ○ & O & O & ○ & O \\
\hline $\begin{array}{r}\text { People walking in the } \\
\text { cycle track }\end{array}$ & O & O & O & O & 0 & O & O \\
\hline $\begin{array}{r}\text { People standing in } \\
\text { the cycle track while } \\
\text { waiting to cross the } \\
\text { street }\end{array}$ & O & O & O & O & O & 0 & ○ \\
\hline $\begin{array}{l}\text { Bicyclists traveling in } \\
\text { the WRONG direction }\end{array}$ & 0 & 0 & 0 & 0 & 0 & 0 & 0 \\
\hline
\end{tabular}

While riding on the Bluebonnet Lane cycle track, have you ever had collisions or near-collisions with other people or objects on the road?

Yes, a collision

Yes, a near-collision

No, neither

If you have been involved in a collision or near-collision, please indicate what other people or objects were involved.

Check all that apply.

\begin{tabular}{|c|c|c|}
\hline & Collision & Near Collision \\
\hline Another bicyclist & $\square$ & $\square$ \\
\hline A pedestrian & $\square$ & $\square$ \\
\hline A turning car & $\square$ & $\square$ \\
\hline A parking car & $\square$ & $\square$ \\
\hline A parked car & $\square$ & $\square$ \\
\hline A delivery truck & $\square$ & $\square$ \\
\hline A bus & $\square$ & $\square$ \\
\hline $\begin{array}{r}\text { One of the cycle track's plastic } \\
\text { flexposts }\end{array}$ & $\square$ & $\square$ \\
\hline $\begin{array}{r}\text { Other stationary object (please } \\
\text { describe). }\end{array}$ & $\square$ & $\square$ \\
\hline
\end{tabular}


Please provide a brief description of the collision(s) you were involved in while riding a bicycle in the cycle track.

\section{Overall Impressions of the Cycle Track}

What is the best thing about the cycle track?

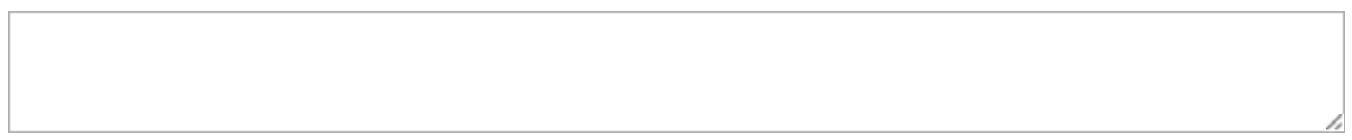

What should be improved about the cycle track?

\section{About Zilker Elementary School}

Do you have a child (or children) that attends Zilker Elementary School?
No
Yes (If yes, what age(s)):

We would like to know about if your child walks or bikes to Zilker Elementary School.

My child or children ...

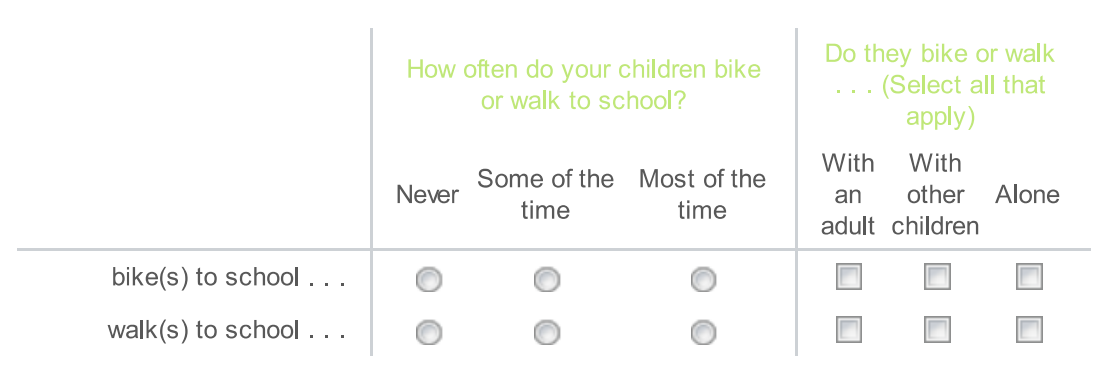

Does your child or children bike or walk on Bluebonnet Lane?
Yes, bikes
Yes, walks
Yes, walks and bikes
No 
Please indicate your level of agreement with the following statements:

Since the cycle track on Bluebonnet Lane was built:

\begin{tabular}{|c|c|c|c|c|c|}
\hline & $\begin{array}{l}\text { Strongly } \\
\text { Disagree }\end{array}$ & $\begin{array}{l}\text { Somewhat } \\
\text { Disagree }\end{array}$ & $\begin{array}{l}\text { Somewhat } \\
\text { Agree }\end{array}$ & Strongly Agree & No Opinion \\
\hline My children are biking to school more often. & O & $\bigcirc$ & O & ○ & ○ \\
\hline My children are walking to school more often. & ○ & ○ & O & ○ & ○ \\
\hline Biking on Bluebonnet Lane is safer for children now. & O & O & O & ○ & O \\
\hline Walking on Bluebonnet Lane is safer for children now. & O & ○ & O & ○ & ○ \\
\hline $\begin{array}{r}\text { The cycle track is an important reason for why I allow } \\
\text { my child to bike on Bluebonnet Lane. }\end{array}$ & O & 0 & 0 & O & O \\
\hline $\begin{array}{l}\text { The cycle track is an important reason for why I allow } \\
\text { my child to walk on Bluebonnet Lane. }\end{array}$ & ○ & ○ & O & ○ & ○ \\
\hline
\end{tabular}

\section{About Bicycling and Comfort:}

Whether or not you currently bicycle in all the following situations, we would like you to consider how comfortable you would be riding a bicycle in each place:

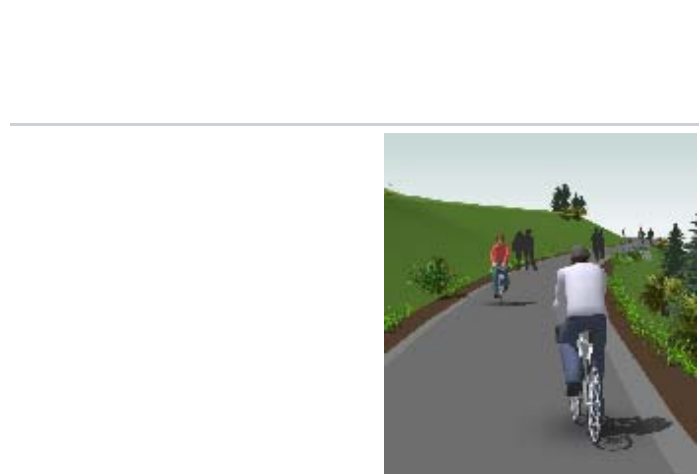

(A) On a path or trail separate from the street

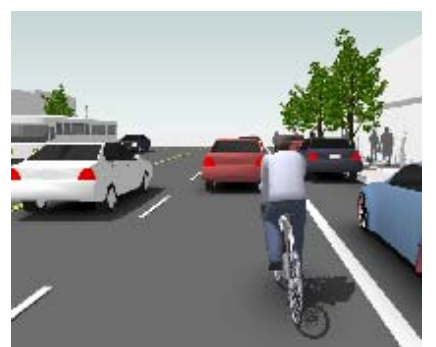

(B) On a commercial street with two lanes of traffic in each direction, with traffic speeds of 35 miles per hour, on-street car parking, and no bike lane

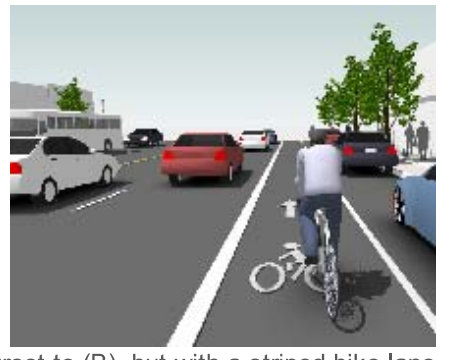

(C) On a similar street to (B), but with a striped bike lane
Very

Uncomfortable

(1)

(2)

(3)

(4)

(5)

Comfor

comfortable

(6)

Very

Uncomfortable

(1)

(2)

(3)

(4)

(5)

Very Comfortable

(6) 
(D) On a similar street to (B), but with a physically separated bike lane

How comfortable would you feel bicycling on a commercial street with two lanes of traffic in each direction, with traffic speeds of 35 miles per hour (Situation D above), but with the following types of separation from traffic:

Very

Uncomfortable

(1)

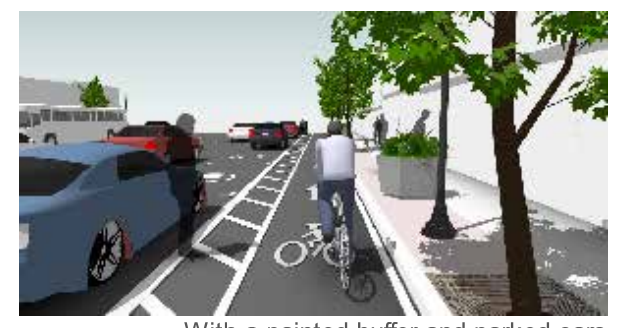

.. With a painted buffer and parked cars

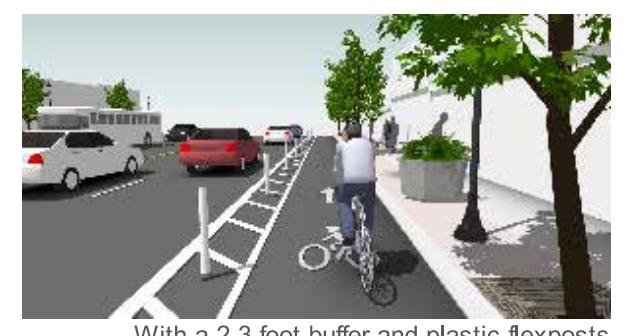

... With a 2-3 foot buffer and plastic flexposts

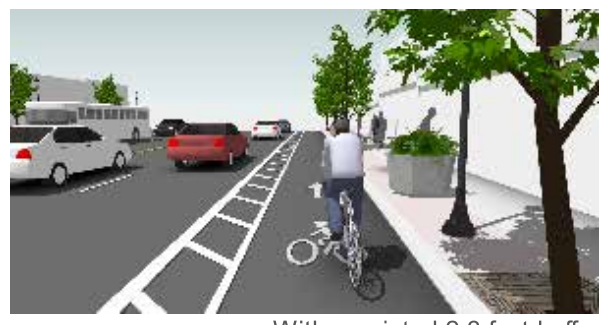

... With a painted 2-3 foot buffer

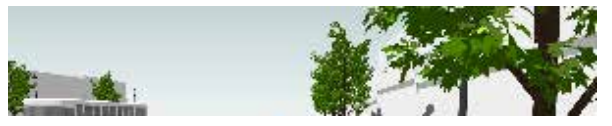

Very

Uncomfortable

(1)

(2)

(3)

(4)

(5)

Very Comfortable

(6) 


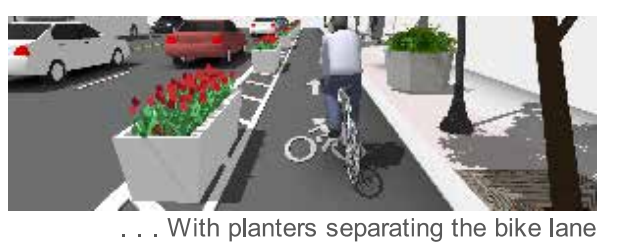

\section{Quatrics Survey Software}
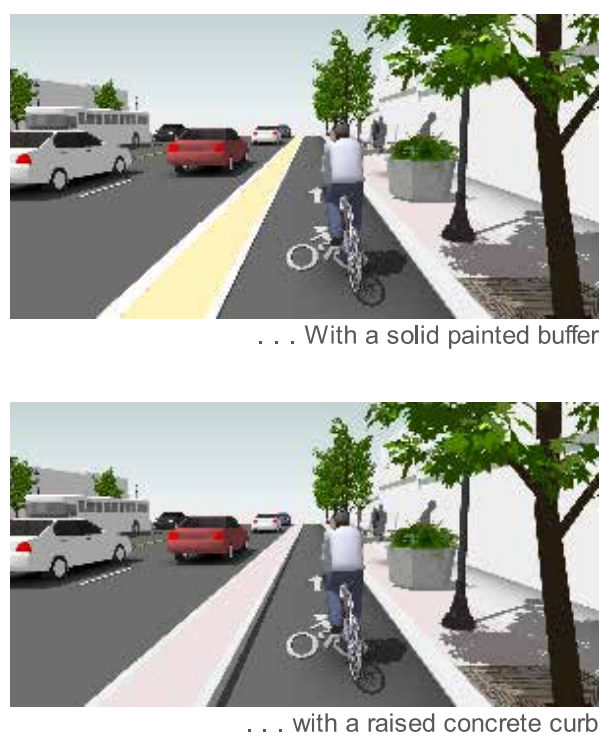

\section{About People You Encounter on the Street:}

Please indicate if you agree or disagree with the following statements:

\begin{tabular}{|c|c|c|c|c|c|}
\hline & $\begin{array}{l}\text { Strongly } \\
\text { Disagree }\end{array}$ & $\begin{array}{l}\text { Somewhat } \\
\text { Disagree }\end{array}$ & Somewhat Agree & Strongly Agree & No Opinion \\
\hline Most drivers follow the rules of the road. & O & ○ & ○ & ○ & 0 \\
\hline Most drivers are predictable. & ○ & O & O & O & 0 \\
\hline Most bicyclists follow the rules of the road. & ○ & ○ & ○ & ○ & O \\
\hline Most bicyclists are predictable. & ○ & 0 & ○ & ○ & ○ \\
\hline Most pedestrians follow the rules of the road. & ○ & 0 & 0 & ○ & O \\
\hline Most pedestrians are predictable. & 0 & 0 & 0 & 0 & 0 \\
\hline
\end{tabular}

\section{About You}

We have a few questions about you so $t$ hat we may understand the characteristics of our survey respondents.

Do you have a current:

\begin{tabular}{l|cc} 
& Yes & No \\
\hline Driver's License & $\bigcirc$ & $\bigcirc$ \\
Transit Pass & $\bigcirc$
\end{tabular}

How many working motor vehicles does your household own or lease? (Do not include motorhomes). 


$$
\begin{array}{r}
\text { \# Vehicles } \\
\text { (Enter } 0 \text { if None) }
\end{array}
$$

How many working adult bicycles does your household own?

$$
\begin{gathered}
\text { \# Bicycles } \\
\text { (Enter } 0 \text { if None) }
\end{gathered}
$$

What is your home zip code?

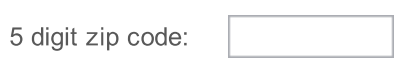

INCLUDING YOURSELF, how many people live in your household?

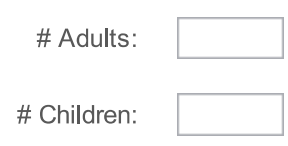

What is your gender?

$$
\text { Male }
$$

○
Female

○

\section{What is your age?}

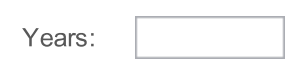

Do you consider yourself: (select all that apply)

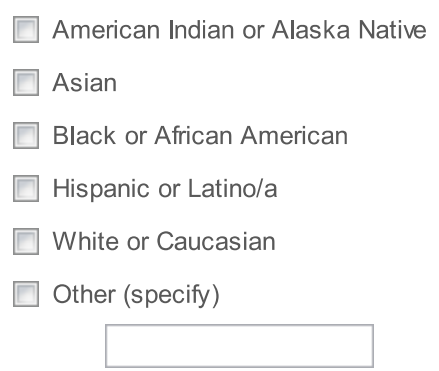

What is your employment status? (Select all that apply)

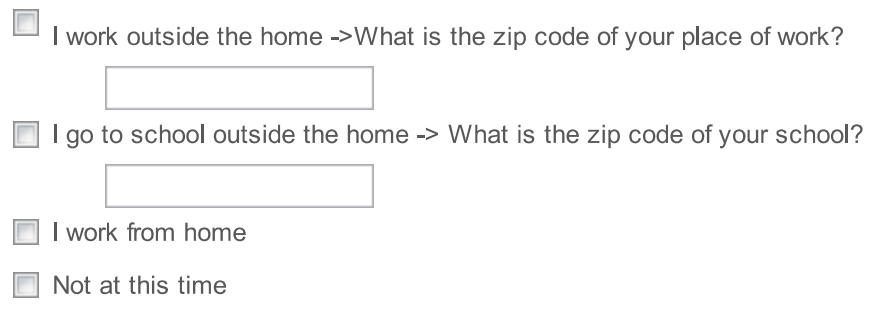


What is your annual household income?
Less than $\$ 25,000$
$\$ 25,000$ to less than $\$ 50,000$
- $\$ 50,000$ to less than $\$ 75,000$
$\$ 75,000$ to less than $\$ 100,000$
$\$ 100,000$ to less than $\$ 200,000$
$\$ 200,000$ or more
I prefer not to provide this information

How many years of school have you completed?
Some high school or less
High school diploma or GED
Some College
Trade/Vocational School
Associate Degree
Four-year college degree or more
Other (please specify):

Is there anything else that you would like to tell us?

That's all the questions we have. Would you like to be entered into a drawing for one of three $\$ 100$ gift cards to Amazon.com?

(2) Yes, I would like to be entered in the drawing ('III enter my name on the next page).

No thanks. I will complete my survey by clicking the ">>" button below

Enter your name and some way for us to get a hold of you below. We will only use this information to contact you if your entry is selected to receive a gift card. This information will not be associated to any of your survey responses.
Name
Phone or email

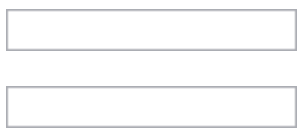

Thanks for taking the survey - please make sure to click the ">>" button below to submit your entry and close the survey! 


\section{BICYCLIST: AUSTIN - RIO GRANDE}




\section{Neighborhood Street Study}

Default Question Block

Dear Bicyclist,

My name is Chris Monsere and I am a faculty member at Portland State University in the Department of Civil and Environmental Engineering. I am the principal investigator on a project researching separated bicycle lanes in six cities across the country.

Hearing from bicyclists like yourself is a very important part of this study. We are only sampling a select number of bicyclists near the Rio Grande Street cycle track. Therefore, every response is very important and we hope you will participate. We will share our findings with the City of Austin and hope that the results will help in future plans for improving bicycling in cities around the United States.

The survey, which starts on the next page, should take about 10-20 minutes. Your participation in the study is voluntary and you may stop taking the survey at any time with no consequences.

The postcard that you received has a unique number that only identifies where and when we handed you the postcard. We will protect the confidentiality of your individual survey responses. None of your responses will be linked to your name or other identifying personal information.

To say thank you, all people who complete their survey by July 5, 2013 will be entered into a drawing for one of three $\$ 100$ Amazon.com gift cards. You will be asked to enter your name and a way to contact you at the end of the survey - this information is voluntary and will not be connected to your survey data.

If you have concerns or problems about your participation in this study or your rights as a research subject, please contact the Human Subjects Research Review Committee, Office of Research and Strategic Partnerships, Market Center Building Suite 620, Portland State University, (877480-4400). If you have questions about the study itself, please contact our research team directly at streets@pdx.edu or 503-725-2875.

Sincerely,

Christopher M. Monsere, Ph.D., P.E., Associate Professor \& Associate Chair Civil and Environmental Engineering Portland State University

Do you agree to participate in this survey?
No
Yes

When you received the postcard for this survey, where were you ...

\begin{tabular}{c|ccc|c|} 
& Place & Can you provide an address or \\
closest intersection to this place?
\end{tabular}

If you selected other, please explain: 


\section{$\underline{\text { Rio Grande Cycle Track }}$}

A separated two-way bikeway, also known as a "cycle track," was recently built on Rio Grande Street from Martin Luther King Jr. Boulevard to 24th Street. To do this, the left-side parking/travel lane that existed before was converted to a two-way bicycle lane with a painted "buffer" area and plastic "flexposts" separating the cycle track from the standard traffic lane (see pictures below). The questions on the following pages are related to this cycle track.

Rio Grande Street before and now:
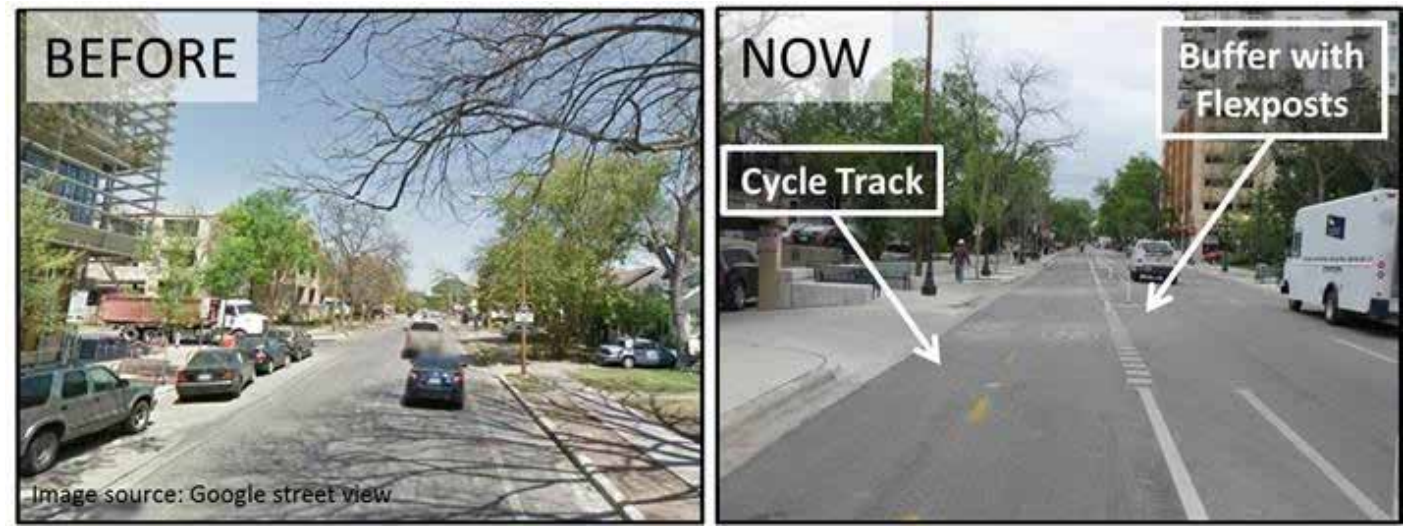

Extent of Rio Grande Street cycle track:

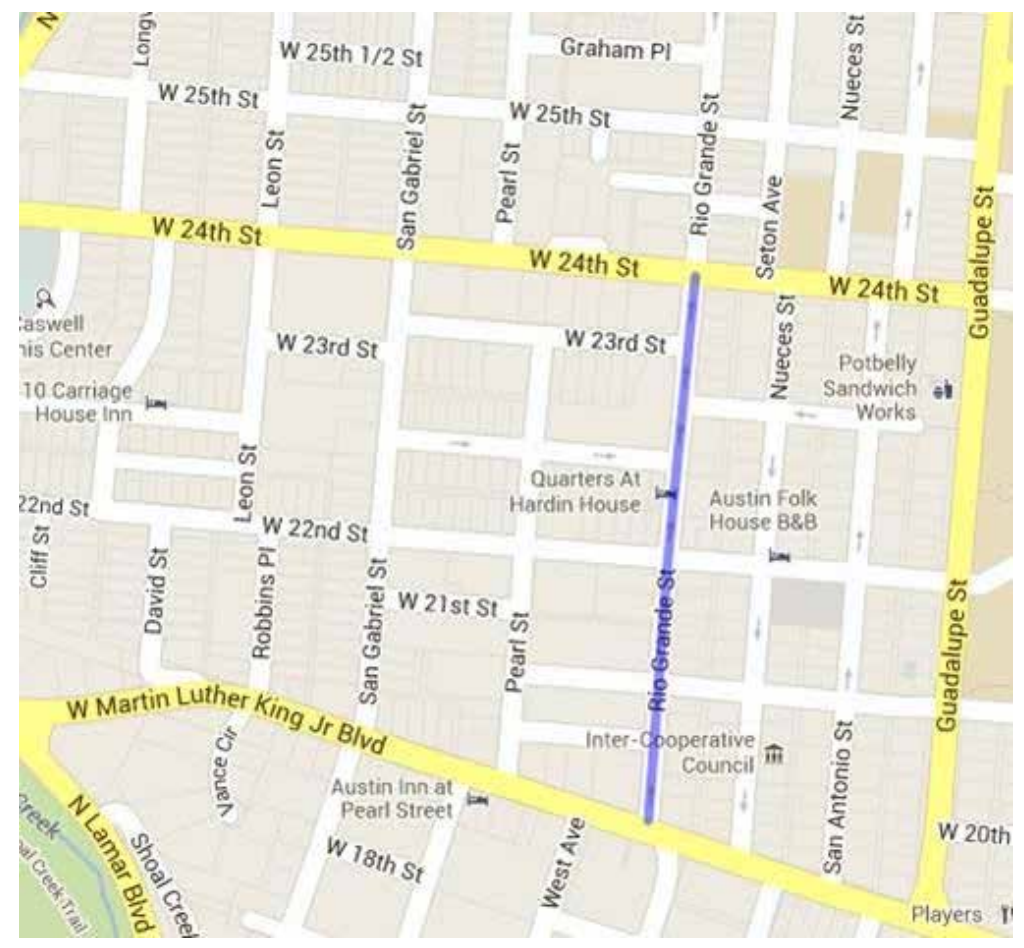

How often do you ride a bicycle on this part of Rio Grande Street?
This was my first time
Less than once a month
A few times a month
A few times a week
Daily 
How comfortable do you feel when bicycling in the Rio Grande Street cycle track in the lane closest to the sidewalk (i.e. southbound)?
(1) Very Uncomfortable
(2)
(3)
(4)
(5)
(6) Very Comfortable

How comfortable do you feel when bicycling in the Rio Grande Street cycle track in the lane closest to the standard traffic lanes and plastic flexposts (i.e. northbound)?
(1) Very Uncomfortable
(2)
(3)
(4)
(5)
(6) Very Comfortable

Since the Rio Grande Street cycle track was built, do you travel on this route:
Less frequently
More frequently
About the same
This is my first time on this route

When bicycling on Rio Grande Street between Martin Luther King Jr. Boulevard and 24th Street, please indicate how often you ride on the following:

\begin{tabular}{l|ccc} 
& Always & Most of the time & Some of the time \\
\hline Cycle track & 0 & 0 & 0 \\
Standard traffic lanes & 0 & 0 & 0 \\
Sidewalk & 0 & 0
\end{tabular}

Consider the trip you were making when you were handed the postcard. Before the Rio Grande Street cycle track was built, how would you have made this trip?

By bicycle, using this same route

By bicycle, using another route - (Please specify which route):
By foot
By public transportation
By car
By taxi
I would NOT have taken this trip
Other (please specify below) 


\section{About Changes due to the Cycle Track}

Because of the Rio Grande Street cycle track ...

\begin{tabular}{|c|c|c|c|c|c|c|}
\hline & $\begin{array}{l}\text { Decreased } \\
\quad \text { a Lot }\end{array}$ & $\begin{array}{l}\text { Decreased } \\
\text { Somewhat }\end{array}$ & $\begin{array}{l}\text { Not } \\
\text { Changed }\end{array}$ & $\begin{array}{l}\text { Increased } \\
\text { Somewhat }\end{array}$ & $\begin{array}{l}\text { Increased } \\
\quad \text { a Lot }\end{array}$ & $\begin{array}{l}\text { No } \\
\text { Opinion }\end{array}$ \\
\hline I feel the safety of bicycling on Rio Grande Street has ... & ○ & $\bigcirc$ & 0 & $\bigcirc$ & O & 0 \\
\hline the time it takes me to bicycle on Rio Grande Street has... & 0 & 0 & 0 & 0 & 0 & 0 \\
\hline $\begin{array}{l}\text { the usefulness of Rio Grande Street for getting to places I want to go } \\
\text { has ... }\end{array}$ & 0 & 0 & 0 & 0 & 0 & 0 \\
\hline how often I stop at shops and businesses on Rio Grande Street has.. & 0 & 0 & 0 & 0 & 0 & 0 \\
\hline how comfortable I feel when bicycling on Rio Grande Street has ... & 0 & 0 & 0 & 0 & 0 & 0 \\
\hline drivers' awareness of people biking on Rio Grande Street has ... & 0 & 0 & 0 & 0 & 0 & 0 \\
\hline drivers' speeds on Rio Grande Street have... . & 0 & 0 & 0 & 0 & 0 & 0 \\
\hline how often I ride a bicycle overall has ... & 0 & 0 & 0 & 0 & 0 & 0 \\
\hline the difficulty of navigating around pedestrians has ... & 0 & 0 & 0 & 0 & 0 & 0 \\
\hline the difficulty of turning across Rio Grande Street while bicycling has . . & 0 & 0 & 0 & 0 & 0 & 0 \\
\hline how comfortable I feel bicycling with my kids has. & 0 & 0 & 0 & 0 & 0 & 0 \\
\hline how safe I feel bicycling with my kids has ... & 0 & 0 & 0 & 0 & 0 & 0 \\
\hline
\end{tabular}

\section{Your Opinions about the Cycle Track}

Please indicate if you agree or disagree with the following statements about the cycle track:

I would go out of my way to ride on Rio Grande Street
compared to other streets.
Taking Rio Grande Street is the most direct bicycle route
to my destination.
The buffer and flexposts between the traffic lanes and the
cycle track make me feel safe.
Leaves and debris in the cycle track are worse than other
places I ride.
The cycle track is wide enough for me to ride comfortably.
The cycle track is wide enough for one bicyclist to pass
another.
The cycle track is wide enough for two people to
comfortably ride side-by-side.

Please indicate if you disagree or agree with the following statements about the effectiveness of the Rio Grande Street cycle track:

\begin{tabular}{|c|c|c|c|c|c|}
\hline & $\begin{array}{l}\text { Strongly } \\
\text { Disagree }\end{array}$ & $\begin{array}{l}\text { Somewhat } \\
\text { Disagree }\end{array}$ & $\begin{array}{l}\text { Somewhat } \\
\text { Agree }\end{array}$ & $\begin{array}{l}\text { Strongly } \\
\text { Agree }\end{array}$ & No Opinion \\
\hline $\begin{array}{l}\text { The cycle track makes it clear where cars can be and } \\
\text { where the designated bicycle lanes are. }\end{array}$ & 0 & 0 & 0 & 0 & $\bigcirc$ \\
\hline $\begin{array}{l}\text { The intersection signals, signs, and street markings } \\
\text { make it clear who has the right-of-way at intersections. }\end{array}$ & ○ & 0 & 0 & $\bigcirc$ & 0 \\
\hline $\begin{array}{lll}- & & \cdots\end{array}$ & & & & & \\
\hline
\end{tabular}


The buffer and flexposts effectively separate bikes from
cars.

The buffer and flexposts do a good job at protecting bikes from cars.

The cycle track makes drivers and bicyclists more predictable.

The cycle track makes it clear where bicyclists and pedestrians should be.

The cycle track design effectively separates bicyclists from pedestrians.

\section{About What You Encounter in the Cycle Track}

Please indicate how often you have observed the following on your rides in the Rio Grande Street cycle track, AND whether you think this is a problem that needs to be addressed

\begin{tabular}{|c|c|c|c|c|c|c|c|}
\hline & \multicolumn{4}{|c|}{$\begin{array}{l}\text { How often do you encounter the following } \\
\qquad \text { IN the CYCLE TRACK? }\end{array}$} & \multicolumn{3}{|c|}{ How much of a problem is this? } \\
\hline & Never & Rarely & Sometimes & Often & $\begin{array}{l}\text { Not a } \\
\text { problem }\end{array}$ & $\begin{array}{l}\text { Minor } \\
\text { problem }\end{array}$ & $\begin{array}{l}\text { Major } \\
\text { Problem }\end{array}$ \\
\hline Cars parking & 0 & 0 & 0 & 0 & 0 & 0 & 0 \\
\hline $\begin{array}{l}\text { Cars loading or } \\
\text { unloading passengers }\end{array}$ & 0 & 0 & 0 & $\bigcirc$ & 0 & 0 & 0 \\
\hline $\begin{array}{l}\text { Delivery vehicles } \\
\text { loading or unloading }\end{array}$ & 0 & 0 & 0 & $\bigcirc$ & 0 & 0 & 0 \\
\hline Taxis & $\bigcirc$ & 0 & 0 & 0 & $\bigcirc$ & 0 & 0 \\
\hline Cars/trucks driving & $\bigcirc$ & 0 & 0 & 0 & 0 & 0 & 0 \\
\hline $\begin{array}{l}\text { Cars/trucks waiting to } \\
\text { make turns OFF of } \\
\text { Rio Grande Street }\end{array}$ & 0 & 0 & 0 & 0 & 0 & 0 & 0 \\
\hline $\begin{array}{r}\text { Cars/trucks waiting to } \\
\text { pull out ONTO Rio } \\
\text { Grande Street }\end{array}$ & 0 & 0 & 0 & 0 & 0 & 0 & 0 \\
\hline $\begin{array}{r}\text { People walking in the } \\
\text { cycle track }\end{array}$ & $\bigcirc$ & 0 & 0 & 0 & $\bigcirc$ & 0 & 0 \\
\hline $\begin{array}{r}\text { People standing in } \\
\text { the cycle track while } \\
\text { waiting to cross the } \\
\text { street }\end{array}$ & 0 & 0 & 0 & ○ & 0 & 0 & 0 \\
\hline $\begin{array}{l}\text { Bicyclists traveling in } \\
\text { the WRONG direction }\end{array}$ & 0 & 0 & 0 & 0 & 0 & 0 & 0 \\
\hline
\end{tabular}

While riding on the Rio Grande Street cycle track, have you ever had collisions or near-collisions with other people or objects on the road?
$\square$ Yes, a collision
$\square$ Yes, a near-collision
$\square$ No, neither

If you have been involved in a collision or near-collision, please indicate what other people or objects were involved.

Check all that apply.

\begin{tabular}{|c|c|c|}
\hline & Collision & Near Collision \\
\hline Another bicyclist & $\square$ & $\square$ \\
\hline A pedestrian & $\square$ & $\square$ \\
\hline A turning car & $\square$ & $\square$ \\
\hline A parking car & $\square$ & $\square$ \\
\hline A parked car & $\square$ & $\square$ \\
\hline
\end{tabular}


A delivery truck

A bus

A taxi

One of the cycle track's plastic flexposts

A stationary object (please describe).

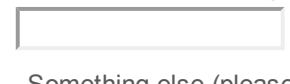

Something else (please describe).

Please provide a brief description of the collision(s) you were involved in while riding a bicycle in the cycle track.

\section{About the MLK Intersection}

A designated bicycle lane for bicyclists traveling northbound (toward the cycle track) on Rio Grande Street has been striped through the Martin Luther King Jr. Boulevard intersection (see picture below). The following questions are about this intersection.

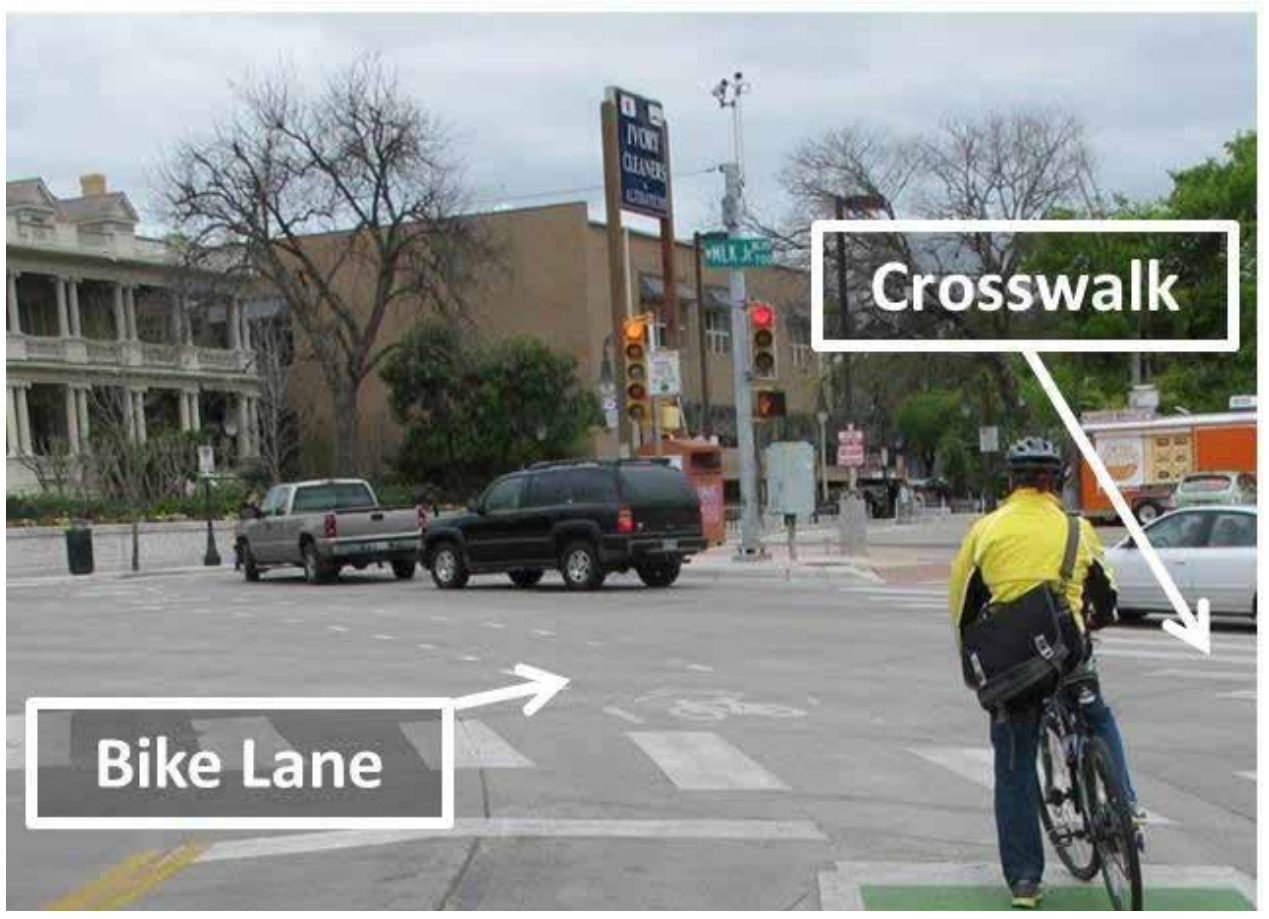


Have you ridden through this intersection in this direction since the cycle track was built?
Yes
No

When riding northbound through this intersection (toward the cycle track - as shown in the photo), I ride in the ...

\begin{tabular}{l|ccc} 
& Always & Most of the time & Some of the time \\
Bike Lane & 0 & 0 & Never \\
Crosswalk & 0 & 0 & 0
\end{tabular}

How comfortable do you feel bicycling through this intersection in the bike lane?
(1) Very Uncomfortable
(2)
(3)
(4)
(5)
(6) Very Comfortable

How satisfied are you with...

\begin{tabular}{l|ccc} 
& Very Dissatisfied $\quad$ Somewhat Dissatisfied & Somewhat Satisfied \\
The amount of time you have to \\
wait for the signal to turn green
\end{tabular}

A bicycle signal has been put in place for bicyclists traveling southbound (away from the cycle track) in the Rio Grande Street cycle track when crossing Martin Luther King Jr. Boulevard (see picture below). The following questions are about this intersection.

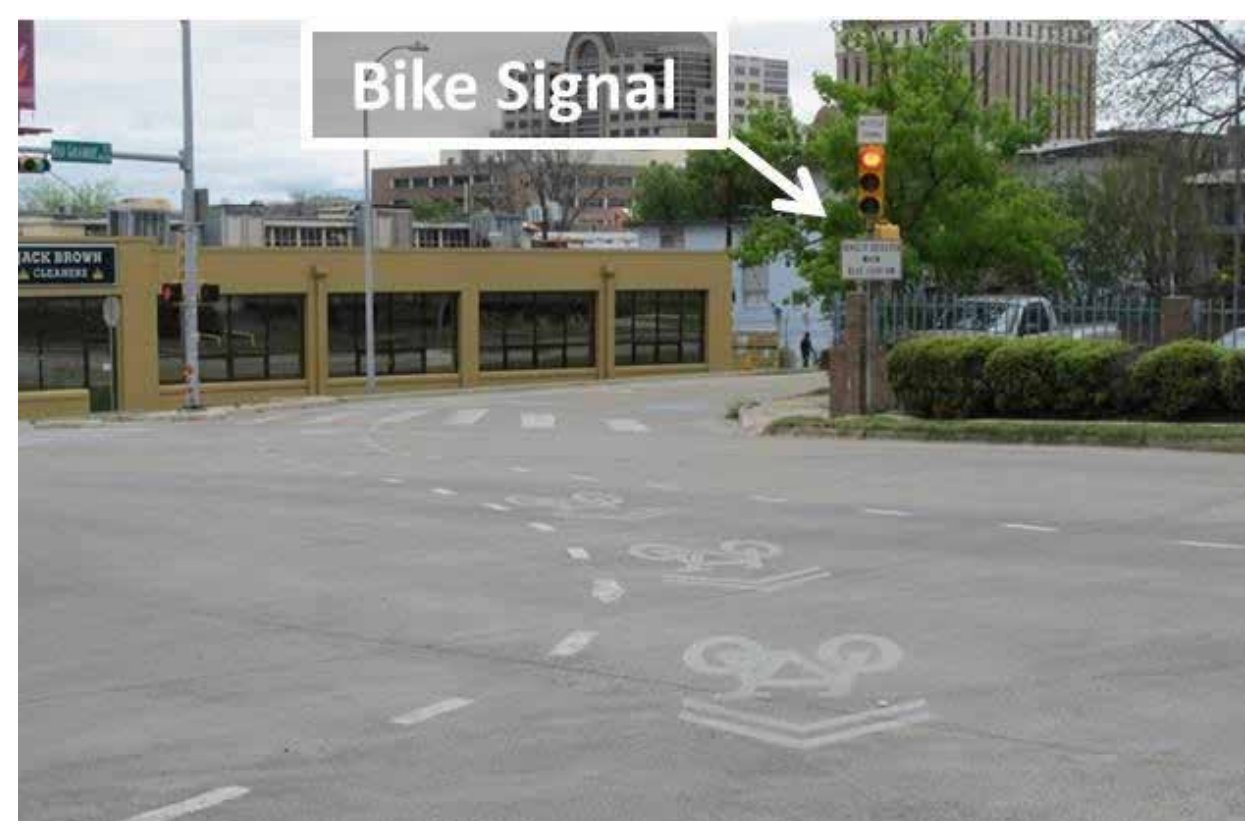




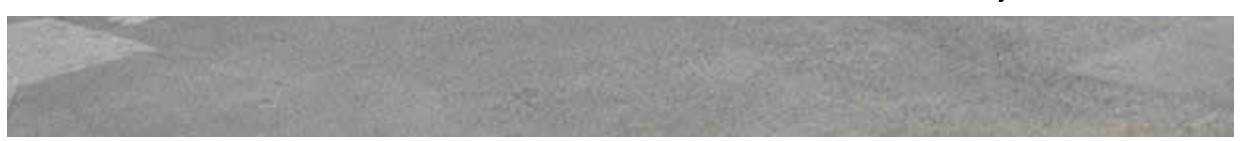

Have you ridden through this intersection in this direction since the cycle track was built?
Yes
No

How comfortable do you feel bicycling through this intersection in this direction?
(1) Very Uncomfortable
(2)
$(3)$
(4)
$\bigcirc(5)$
(6) Very Comfortable

How often do you wait for the bicycle signal to turn green before crossing MLK?
Always
Most of the time
Some of the time
Never
I did not know there was a bicycle signal there

How satisfied are you with...

\begin{tabular}{l|l|} 
& Very Dissatisfied $\quad$ Somewhat Dissatisfied Somewhat Satisfied \\
\hline The amount of time you have to & \\
wait for the bicycle signal to turn \\
green \\
How long the bicycle signal \\
stays green while you are \\
crossing
\end{tabular}

How often are the following blocking your way through the intersection when the bicycle signal is green:

\begin{tabular}{l|cc} 
& Never & Some of the time \\
\hline Cars/trucks & 0 & 0 \\
Pedestrians & 0 & 0
\end{tabular}

\section{Overall Impressions of the Cycle Track}

What is the best thing about the cycle track? 
What should be improved about the cycle track?

\section{About Bicycling and Comfort}

Regardless of whether you currently bicycle in all the following situations, please consider how comfortable you would be riding a bicycle in each place:

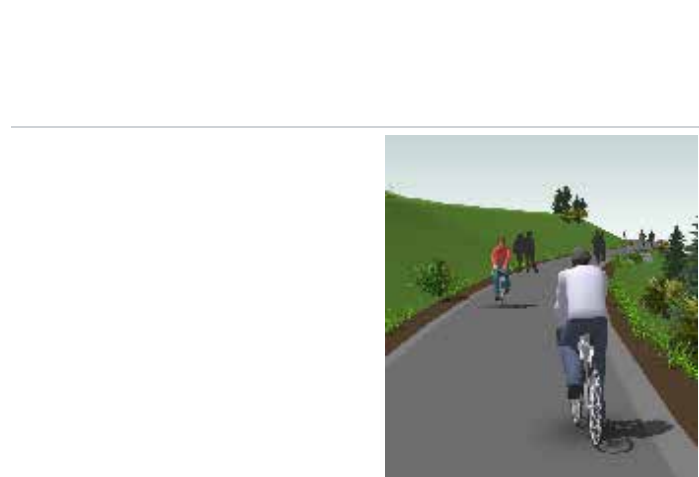

(A) On a path or trail separate from the street

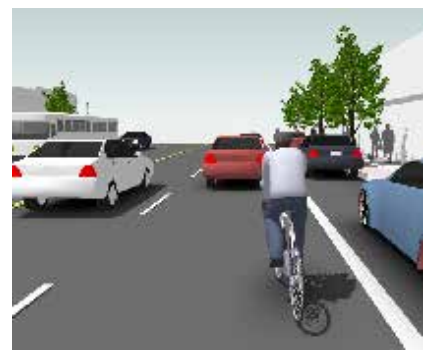

(B) On a commercial street with two lanes of traffic in each direction, with traffic speeds of 35 miles per hour, on-street car parking, and no bike lane

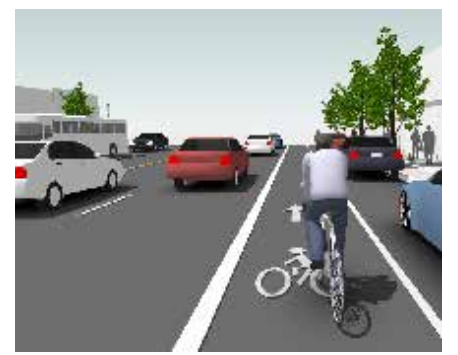

(C) On a similar street to (B), but with a striped bike lane added

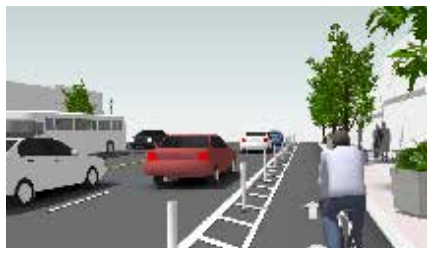

Very Comfortable Uncomfortable

(1)

(2)

(3)

(4)

(5)

(6)

Very

Very

Uncomfortable

(1)

(2)

(3)

(4)

(5)

Comfortable

(6) 
(D) On a similar street to (B), but with a physically separated bike lane

How comfortable would you feel bicycling on a commercial street with two lanes of traffic in each direction, with traffic speeds of 35 miles per hour (Situation $D$ above), but with the following types of separation from traffic:
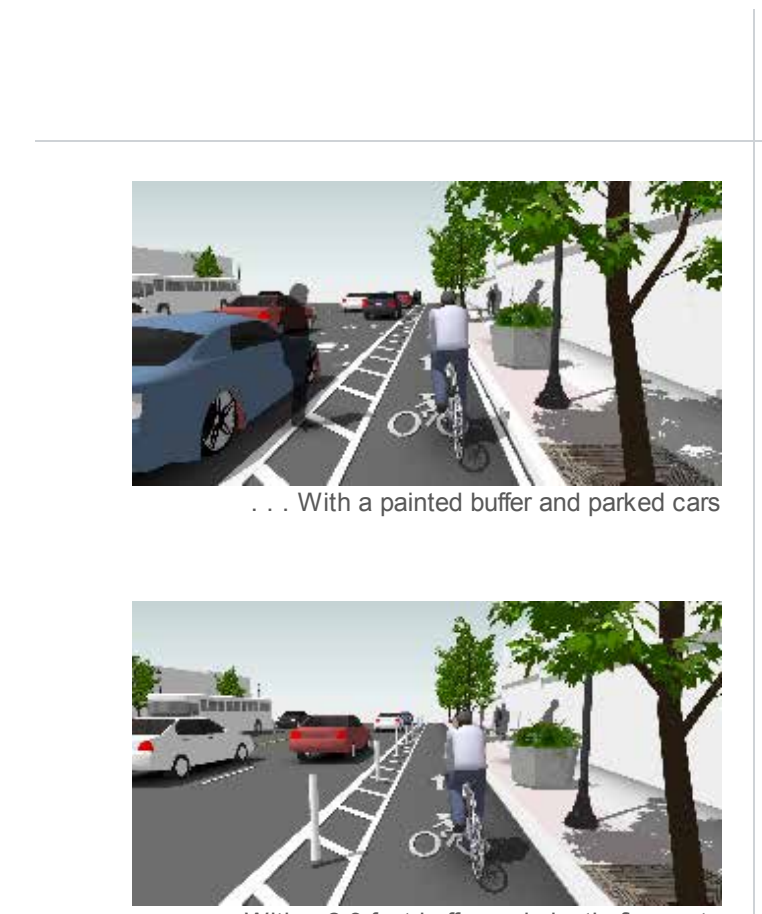

... With a 2-3 foot buffer and plastic flexposts
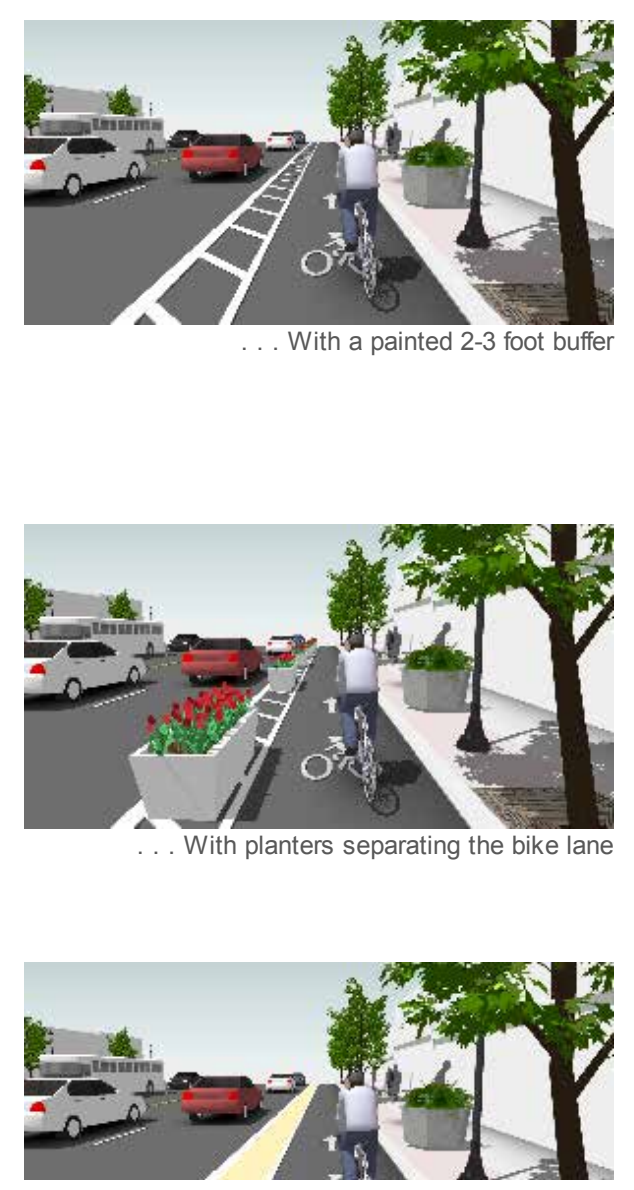

Very

Uncomfortable

(1)

(2)
Very

Uncomfortable

(1)

(3)

(4)

(5)

Very comfortable

(6)

(2)

(3)
(4)

(5)
Very Comfortable

(6) 

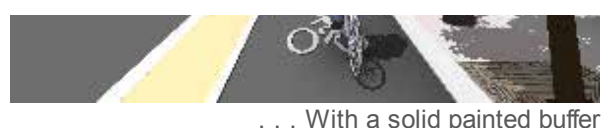

... With a solid painted buffer

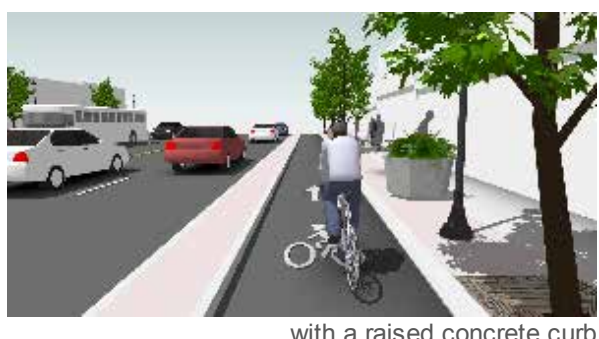

1

... with a raised concrete curb

\section{About People you Encounter on the Street}

Please indicate if you agree or disagree with the following statements:

\begin{tabular}{|c|c|c|c|c|c|}
\hline & $\begin{array}{l}\text { Strongly } \\
\text { Disagree }\end{array}$ & $\begin{array}{l}\text { Somewhat } \\
\text { Disagree }\end{array}$ & Somewhat Agree & Strongly Agree & No Opinion \\
\hline Most drivers follow the rules of the road. & 0 & 0 & 0 & 0 & 0 \\
\hline Most drivers are predictable. & $\bigcirc$ & 0 & 0 & 0 & 0 \\
\hline Most bicyclists follow the rules of the road. & 0 & 0 & $\bigcirc$ & 0 & 0 \\
\hline Most bicyclists are predictable. & 0 & 0 & 0 & 0 & 0 \\
\hline Most pedestrians follow the rules of the road. & 0 & 0 & 0 & 0 & 0 \\
\hline Most pedestrians are predictable. & 0 & 0 & 0 & 0 & 0 \\
\hline
\end{tabular}

\section{$\underline{\text { About You }}$}

We have a few questions about you so that we may understand the characteristics of our survey respondents.

Do you have a current:

\begin{tabular}{l|cc} 
& Yes No \\
Driver's License & 0 & 0 \\
Transit Pass & 0
\end{tabular}

How many working motor vehicles does your household own or lease?

(Do not include motorhomes).

$$
\begin{array}{r}
\text { \# Vehicles } \\
\text { (Enter } 0 \text { if None) }
\end{array}
$$

How many working adult bicycles does your household own?

$$
\text { \# Adult bicycles }
$$$$
\text { (Enter } 0 \text { if None) }
$$ 
INCLUDING YOURSELF, how many people live in your household?

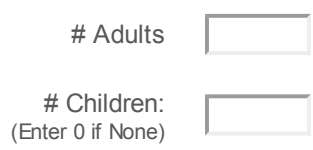

What is your gender?

Male

0
Female

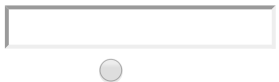

0

What is your age?

$$
\text { Years }
$$

Do you consider yourself: (select all that apply)

American Indian or Alaska Native
Asian
Black or African American
Hispanic or Latino/a
White or Caucasian
Other (specify)

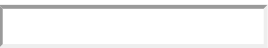

What is your employment status? (Select all that apply)

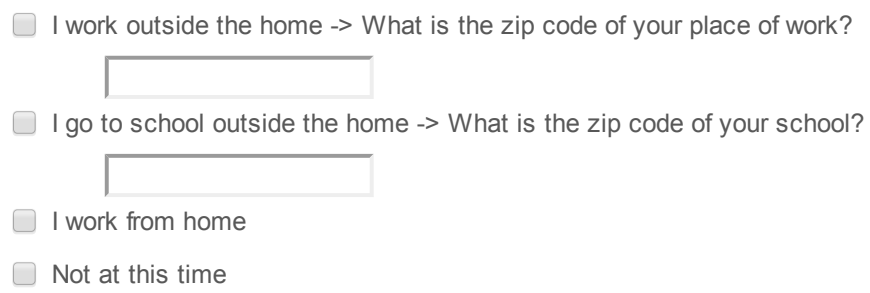

What is your annual household income?
Less than $\$ 25,000$
$\$ 25,000$ to less than $\$ 50,000$
$\$ 50,000$ to less than $\$ 75,000$
$\$ 75,000$ to less than $\$ 100,000$
$\$ 100,000$ to less than $\$ 200,000$
$\$ 200,000$ or more
I nrafor not tn nrniido thic information 
How many years of school have you completed?
Some high school or less
High school diploma or GED
Some College
Trade/Vocational School
Associate Degree
Four-year college degree or more
Other (please specify):

Is there anything else that you would like to tell us?

That's all the questions we have. Would you like to be entered into a drawing for one of three $\$ 100$ gift cards to Amazon.com?

- Yes, I would like to be entered in the drawing (I'll enter my name on the next page).

No thanks. I will complete my survey by clicking the ">>" button

Enter you name and some way for us to get a hold of you below. We will only use this information to contact you in the event that your entry is selected to receive a gift card. This information will not be associated to any of your survey responses.

Name

Phone or email

Thanks for taking the survey - please make sure to click the ">>" button to submit your entry and close the survey! 


\section{Bicyclist: Chicago - Milwaukee Ave.}




\section{Neighborhood Street Study}

Default Question Block

Dear Bicyclist,

My name is Chris Monsere and I am a faculty member at Portland State University in the Department of Civil and Environmental Engineering. I am the principal investigator on a project researching separated bicycle lanes in six cities across the country.

Hearing from bicyclists like yourself is a very important part of this study. We are only sampling a select number of bicyclists near the Milwaukee Avenue protected bike lanes. Therefore, every response is very important and we hope you will participate. We will share our findings with the Chicago Department of Transportation and hope that the results will help in future plans for improving bicycling in cities around the United States.

The survey, which starts on the next page, should take about 10-20 minutes. Your participation in the study is voluntary and you may stop taking the survey at any time with no consequences.

The postcard that you received has a unique number that only identifies where and when we handed you the postcard. We will protect the confidentiality of your individual survey responses. None of your responses will be linked to your name or other identifying personal information.

To say thank you, all people who complete their survey by 10/10/13 will be entered into a drawing for one of three $\$ 100$ Amazon.com gift cards. You will be asked to enter your name and a way to contact you at the end of the survey - this information is voluntary and will not be connected to your survey data.

If you have concerns or problems about your participation in this study or your rights as a research subject, please contact the Human Subjects Research Review Committee, Office of Research and Strategic Partnerships, Market Center Building Suite 620, Portland State University, (877480-4400). If you have questions about the study itself, please contact our research team directly at streets@pdx.edu or 503-725-2875.

Sincerely,

Christopher M. Monsere, Ph.D., P.E., Associate Professor \& Associate Chair Civil and Environmental Engineering Portland State University

Do you agree to participate in this survey?
No
Yes

When you received the postcard for this survey, where were you ...

\begin{tabular}{c|c|ccc|c|c|c|} 
Can you provide an address or \\
closest intersection to this place?
\end{tabular}

If you selected other, please explain: 
What type of bicycle were you riding when you received the postcard?
Personal Bicycle
Diny (Bike Share) Bicycle
Rental Bicycle
Other (please specify):

\section{Milwaukee Avenue Protected Bike Lanes}

A separated bikeway was recently built on North Milwaukee Avenue between North Elston Avenue and West Kinzie Street. Bike lanes were separated from other traffic lanes by a combination of a striped "buffer" zone, plastic "flexposts" or by moving parked cars away from the curb to provided a protected lane (see picture below).

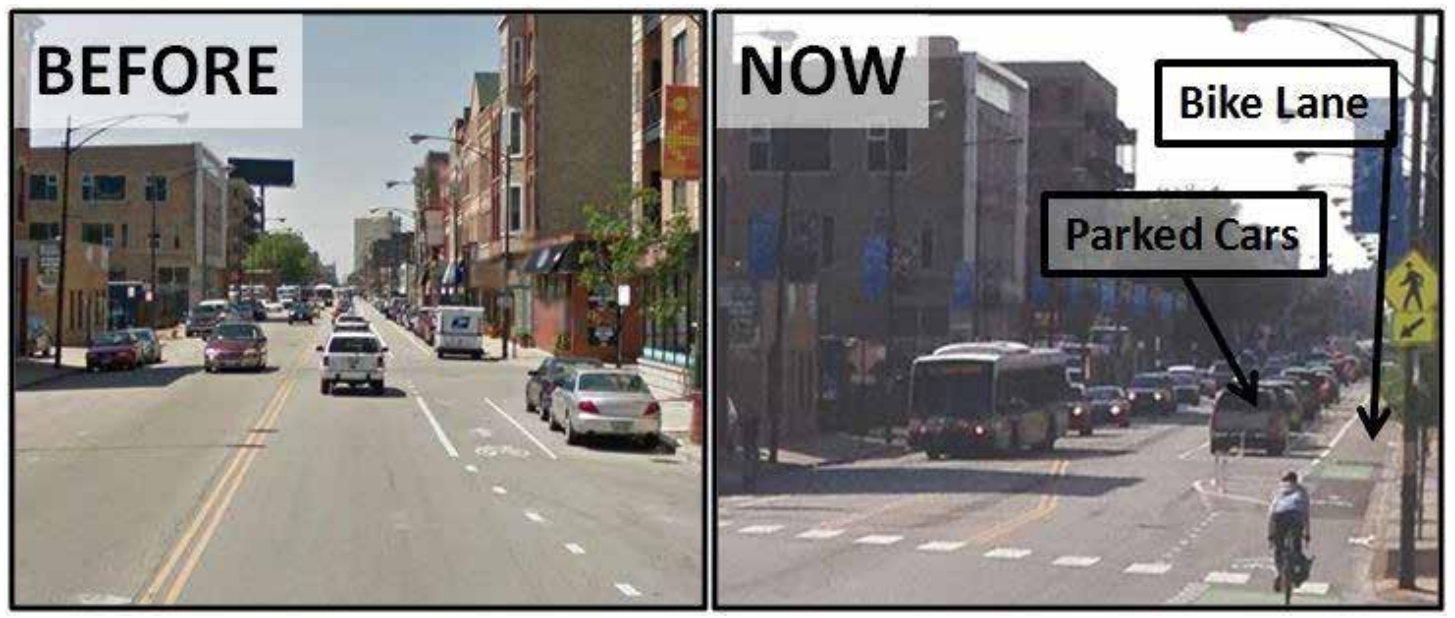

Extent of the Milwaukee Avenue protected bike lanes:

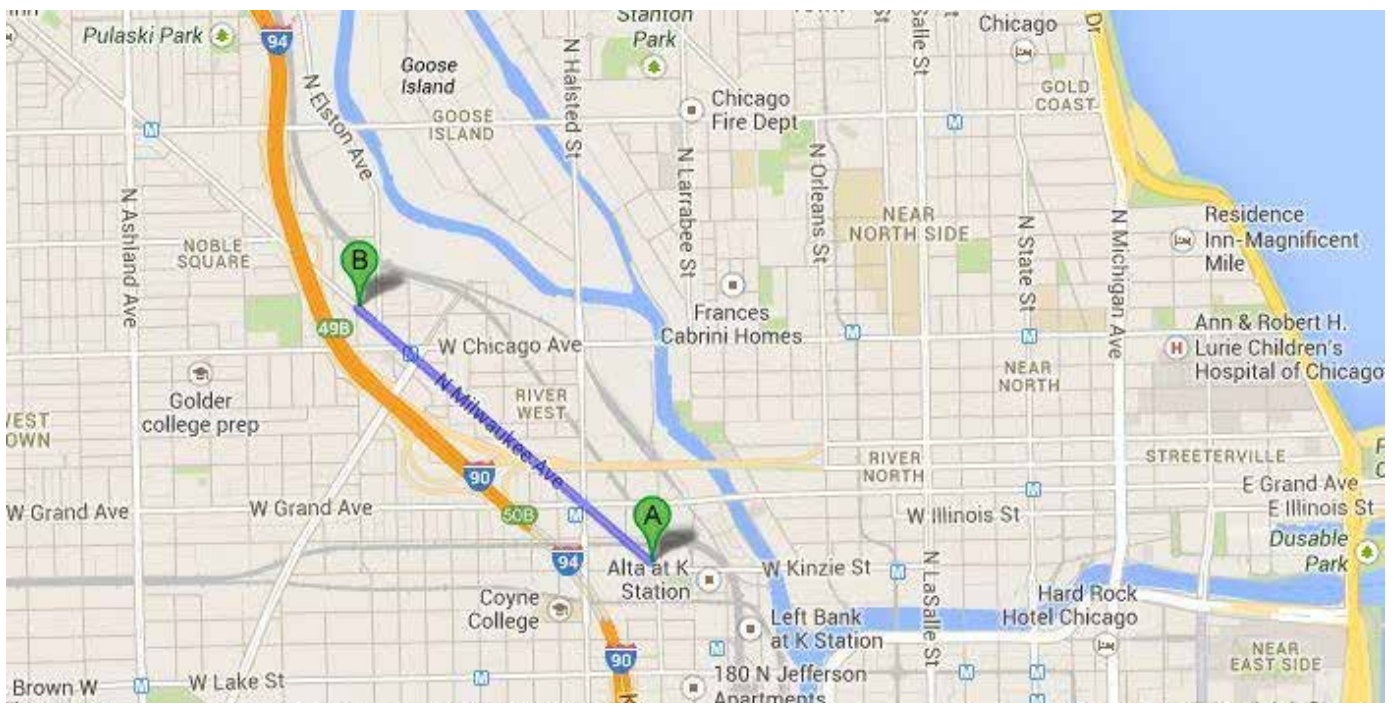




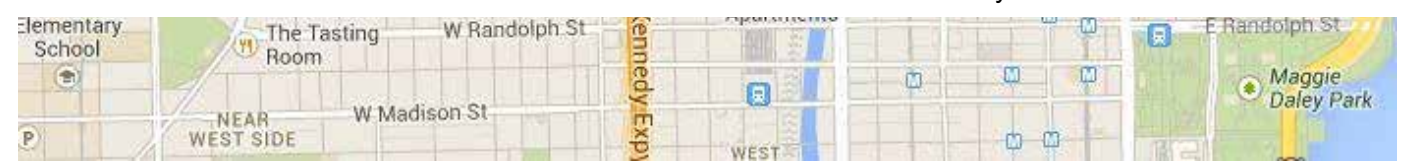

How often do you ride a bicycle on this part of Milwaukee Avenue?
This was my first time
Less than once a month
A few times a month
A few times a week
Daily

How comfortable do you feel when bicycling in (different sections of) the Milwaukee Avenue protected bike lanes?

\begin{tabular}{|c|c|c|c|c|c|c|}
\hline & $\begin{array}{c}\text { (1) Very } \\
\text { Uncomfortable }\end{array}$ & (2) & (3) & (4) & (5) & $\begin{array}{l}\text { (6) Very } \\
\text { Comfortable }\end{array}$ \\
\hline $\begin{array}{r}\text { In sections with parked cars } \\
\text { between the bike lane and } \\
\text { moving traffic }\end{array}$ & 0 & 0 & 0 & 0 & 0 & 0 \\
\hline $\begin{array}{l}\text { In sections with plastic flexposts } \\
\text { marking the separation between } \\
\text { the bike lane and moving traffic }\end{array}$ & 0 & 0 & 0 & 0 & 0 & 0 \\
\hline $\begin{array}{r}\text { In sections with a painted area } \\
\text { separating the bike lane and } \\
\text { moving traffic }\end{array}$ & 0 & 0 & 0 & 0 & 0 & 0 \\
\hline
\end{tabular}

Since the Milwaukee Avenue protected bike lanes were built, do you travel on this route:
Less frequently
More frequently
About the same
This is my first time on this route

When bicycling on Milwaukee Avenue between Elston Avenue and Kinzie Street, how often you ride on the following:

\begin{tabular}{l|cccc} 
& Always & Most of the time & Some of the time & Never \\
\hline Protected bike lanes & 0 & 0 & 0 & 0 \\
Standard traffic lanes & 0 & 0 & 0 & 0 \\
Sidewalk & 0 & 0 & 0
\end{tabular}

Consider the trip you were making when you were handed the postcard. Before the Milwaukee Avenue protected bike lanes were built, how would you have made this trip?

By bicycle, using this same route

By bicycle, using another route - (Please specify which route):

By foot

By public transportation

By car

- - 
By taxi

I would NOT have taken this trip

Other (please specify below)

\section{About Changes due to the Protected Bike Lanes}

Because of the Milwaukee Avenue protected bike lanes...

\begin{tabular}{|c|c|c|c|c|c|c|}
\hline & $\begin{array}{l}\text { Decreased } \\
\quad \text { a Lot }\end{array}$ & $\begin{array}{l}\text { Decreased } \\
\text { Somewhat }\end{array}$ & $\begin{array}{c}\text { Not } \\
\text { Changed }\end{array}$ & $\begin{array}{l}\text { Increased } \\
\text { Somewhat }\end{array}$ & $\begin{array}{l}\text { Increased } \\
\quad \text { a Lot }\end{array}$ & $\begin{array}{l}\text { No } \\
\text { Opinion }\end{array}$ \\
\hline I feel the safety of bicycling on Milwaukee Avenue has ... & O & 0 & 0 & ○ & O & O \\
\hline the time it takes me to bicycle on Milwaukee Avenue has ... & 0 & ○ & O & O & O & O \\
\hline $\begin{array}{l}\text { the usefulness of Milwaukee Avenue for getting to places I want to go } \\
\text { has ... }\end{array}$ & O & $\bigcirc$ & O & O & O & 0 \\
\hline how often I stop at shops and businesses on Milwaukee Avenue has. & O & O & O & O & 0 & O \\
\hline how comfortable I feel when bicycling on Milwaukee Avenue has ... & 0 & ○ & O & ○ & ○ & $\bigcirc$ \\
\hline drivers' awareness of people biking on Milwaukee Avenue has ... & O & O & ○ & $\bigcirc$ & O & ○ \\
\hline drivers' speeds on Milwaukee Avenue have... & ○ & ○ & ○ & ○ & ○ & ○ \\
\hline how often I ride a bicycle overall has ... & ○ & ○ & ○ & ○ & ○ & ○ \\
\hline the difficulty of navigating around turning motor vehicles has ... & 0 & $\bigcirc$ & 0 & ○ & O & O \\
\hline the difficulty of navigating around pedestrians has ... & 0 & $\bigcirc$ & 0 & ○ & 0 & 0 \\
\hline
\end{tabular}

\section{Your Opinions about the Protected Bike Lanes}

Please indicate if you agree or disagree with the following statements about the Milwaukee Avenue protected bike lanes:

\begin{tabular}{|c|c|c|c|c|c|}
\hline & $\begin{array}{l}\text { Strongly } \\
\text { Disagree }\end{array}$ & $\begin{array}{l}\text { Somewhat } \\
\text { Disagree }\end{array}$ & $\begin{array}{l}\text { Somewhat } \\
\text { Agree }\end{array}$ & $\begin{array}{l}\text { Strongly } \\
\text { Agree }\end{array}$ & No Opinion \\
\hline $\begin{array}{l}\text { I would go out of my way to ride on Milwaukee Avenue } \\
\text { compared to other streets. }\end{array}$ & 0 & 0 & 0 & 0 & 0 \\
\hline $\begin{array}{r}\text { Taking Milwaukee Avenue is the most direct bicycle route } \\
\text { to my destination. }\end{array}$ & 0 & 0 & 0 & 0 & 0 \\
\hline $\begin{array}{l}\text { The buffer section with parked cars between the traffic } \\
\text { lanes and the bike lanes makes me feel safe. }\end{array}$ & 0 & 0 & 0 & 0 & 0 \\
\hline \multirow[t]{2}{*}{$\begin{array}{l}\text { The buffer section with plastic flexposts between the } \\
\text { traffic lanes and the bike lanes makes me feel safe. }\end{array}$} & 0 & 0 & 0 & 0 & 0 \\
\hline & $\begin{array}{l}\text { Strongly } \\
\text { Disagree }\end{array}$ & $\begin{array}{l}\text { Somewhat } \\
\text { Disagree }\end{array}$ & $\begin{array}{l}\text { Somewhat } \\
\text { Agree }\end{array}$ & $\begin{array}{l}\text { Strongly } \\
\text { Agree }\end{array}$ & No Opinion \\
\hline $\begin{array}{l}\text { The buffer section with a painted/striped buffer between } \\
\text { the traffic lanes and the bike lanes makes me feel safe. }\end{array}$ & 0 & 0 & 0 & 0 & 0 \\
\hline $\begin{array}{l}\text { Leaves and debris in the protected bike lanes are worse } \\
\text { than other places I ride. }\end{array}$ & 0 & 0 & 0 & 0 & 0 \\
\hline $\begin{array}{l}\text { Puddles and standing water are worse than other places I } \\
\text { ride. }\end{array}$ & 0 & 0 & 0 & 0 & 0 \\
\hline \multirow[t]{2}{*}{$\begin{array}{l}\text { The protected bike lanes are wide enough for me to ride } \\
\text { comfortably. }\end{array}$} & 0 & 0 & 0 & 0 & 0 \\
\hline & $\begin{array}{l}\text { Strongly } \\
\text { Disagree }\end{array}$ & $\begin{array}{l}\text { Somewhat } \\
\text { Disagree }\end{array}$ & $\begin{array}{l}\text { Somewhat } \\
\text { Agree }\end{array}$ & $\begin{array}{l}\text { Strongly } \\
\text { Agree }\end{array}$ & No Opinion \\
\hline $\begin{array}{r}\text { The protected bike lanes are wide enough for one } \\
\text { bicyclist to pass another. }\end{array}$ & 0 & 0 & 0 & 0 & 0 \\
\hline $\begin{array}{l}\text { The protected bike lanes are wide enough for two people } \\
\text { to comfortably ride side-by-side. }\end{array}$ & 0 & 0 & 0 & 0 & 0 \\
\hline $\begin{array}{r}\text { If I am bicycling with another adult, I would prefer to ride } \\
\text { side-by-side. }\end{array}$ & 0 & 0 & 0 & 0 & 0 \\
\hline
\end{tabular}


Please indicate if you disagree or agree with the following statements about the effectiveness of the Milwaukee Avenue protected bike lanes are:

$\begin{gathered}\text { The protected bike lanes make it clear where cars can be } \\ \text { and where the designated bicycle lanes are. }\end{gathered}$
$\begin{gathered}\text { The intersection signals, signs, and street markings } \\ \text { Disagree } \\ \text { make it clear who has the right-of-way at intersections. } \\ \text { The buffer effectively separates bikes from cars. }\end{gathered}$
The buffer does a good job at protecting bikes from cars.
The protected bike lanes make drivers and bicyclists
more predictable.

What do you think it means when the pavement is painted green along the bikeway?

\section{About What You Encounter in the Protected Bike Lanes}

Please indicate how often you have observed the following on your rides in the Milwaukee Avenue protected bike lanes, AND whether you think this is a problem that needs to be addressed

\begin{tabular}{|c|c|c|c|c|c|c|c|}
\hline & \multicolumn{4}{|c|}{$\begin{array}{l}\text { How often do you encounter the following } \\
\text { IN the Protected Bike Lanes? }\end{array}$} & \multicolumn{3}{|c|}{ How much of a problem is this? } \\
\hline & Never & Rarely & Sometimes & Often & $\begin{array}{l}\text { Not a } \\
\text { problem }\end{array}$ & $\begin{array}{l}\text { Minor } \\
\text { problem }\end{array}$ & $\begin{array}{l}\text { Major } \\
\text { Problem }\end{array}$ \\
\hline Cars parking & 0 & 0 & 0 & 0 & 0 & 0 & 0 \\
\hline $\begin{array}{l}\text { Cars loading or } \\
\text { unloading passengers }\end{array}$ & 0 & 0 & 0 & 0 & ○ & 0 & 0 \\
\hline $\begin{array}{l}\text { Delivery vehicles } \\
\text { loading or unloading }\end{array}$ & 0 & 0 & 0 & 0 & 0 & 0 & 0 \\
\hline Taxis & 0 & 0 & 0 & 0 & 0 & 0 & 0 \\
\hline $\begin{array}{r}\text { Cars/trucks driving } \\
\text { where they are not } \\
\text { supposed to (in the } \\
\text { bike lanes) }\end{array}$ & 0 & 0 & 0 & 0 & 0 & 0 & 0 \\
\hline \multirow[t]{2}{*}{$\begin{array}{l}\text { Cars/trucks waiting to } \\
\text { make turns OFF of } \\
\text { Milwaukee Avenue }\end{array}$} & 0 & 0 & 0 & 0 & ○ & 0 & 0 \\
\hline & Never & Rarely & Sometimes & Often & $\begin{array}{l}\text { Not a } \\
\text { problem }\end{array}$ & $\begin{array}{l}\text { Minor } \\
\text { problem }\end{array}$ & $\begin{array}{l}\text { Major } \\
\text { Problem }\end{array}$ \\
\hline $\begin{array}{r}\text { Cars/trucks waiting to } \\
\text { pull out ONTO } \\
\text { Milwaukee Avenue }\end{array}$ & 0 & 0 & 0 & 0 & 0 & 0 & 0 \\
\hline $\begin{array}{l}\text { People walking in the } \\
\text { bike lane }\end{array}$ & 0 & 0 & 0 & 0 & 0 & 0 & 0 \\
\hline $\begin{array}{l}\text { People standing in } \\
\text { the bike lane while } \\
\text { Mritinn tn } n \text { moce tho }\end{array}$ & 0 & 0 & 0 & 0 & ○ & 0 & 0 \\
\hline
\end{tabular}


vvaluily u vivos แ
street

Bicyclists traveling in the WRONG direction

Buses loading and unloading passengers

While riding in the Milwaukee Avenue protected bike lanes, have you had collisions or near-collisions with other people or objects on the road?
Yes, a collision
Yes, a near-collision
No, neither

If you have been involved in a collision or near-collision, please indicate what other people or objects were involved.

Check all that apply.

\begin{tabular}{|c|c|c|}
\hline & Collision & Near Collision \\
\hline Another bicyclist & $\square$ & $\square$ \\
\hline A pedestrian & $\square$ & $\square$ \\
\hline A turning car & $\square$ & $\square$ \\
\hline A parking car & $\square$ & $\square$ \\
\hline A parked car & $\square$ & 回 \\
\hline A delivery truck & 回 & 回 \\
\hline A bus & $\square$ & $\square$ \\
\hline A taxi & $\square$ & $\square$ \\
\hline One of the plastic flexposts & $\square$ & $\square$ \\
\hline $\begin{array}{r}\text { Other stationary object (please } \\
\text { describe). }\end{array}$ & $\square$ & $\square$ \\
\hline $\begin{array}{r}\text { Something else (please } \\
\text { describe). }\end{array}$ & $\square$ & $\square$ \\
\hline
\end{tabular}

Please provide a brief description of the collision(s) you were involved in while riding a bicycle in the protected bike lanes.

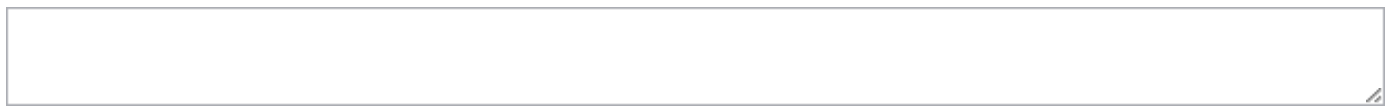

\section{Intersection of N. Milwaukee Avenue with Desplaines/Kinzie}

As you ride toward downtown Chicago (southeast) to the intersection of North Milwaukee Avenue with N. Desplaines and N. Kinzie, there is a bike lane on either side of the standard traffic lane - the green lane to the left directs bicyclists east onto Kinzie, while the bike lane to the right directs cyclists south onto Desplaines (see picture below).

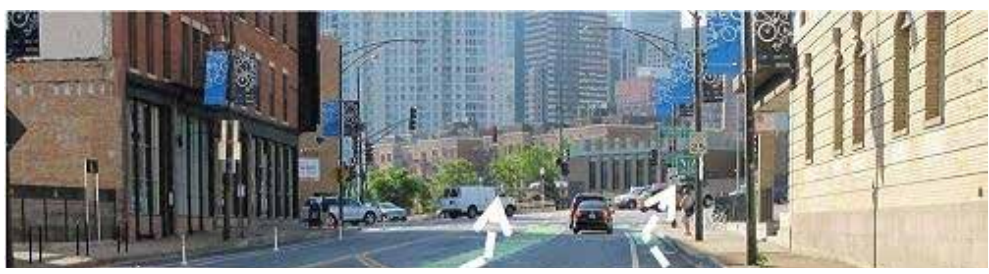




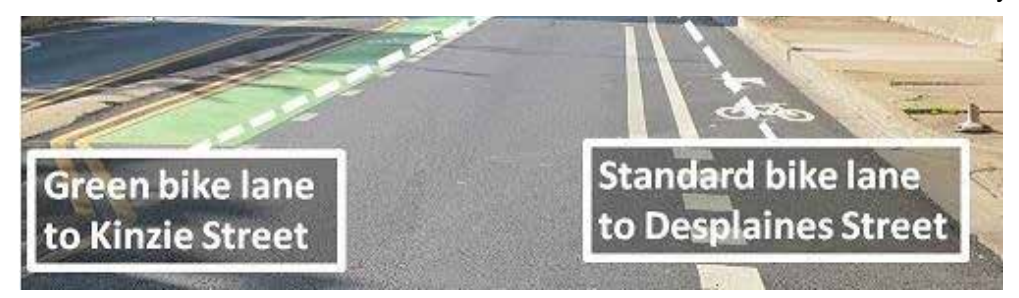

How often have you bicycled through this intersection in the direction of downtown Chicago and made the following turns?

\begin{tabular}{|c|c|c|c|}
\hline & Often & Sometimes & Never \\
\hline $\begin{array}{l}\text { Turning left from N. Milwaukee Avenue onto } \\
\text { Kinzie Street }\end{array}$ & 0 & 0 & 0 \\
\hline $\begin{array}{l}\text { Turning right from N. Milwaukee Avenue } \\
\text { onto Desplaines Street }\end{array}$ & 0 & 0 & 0 \\
\hline
\end{tabular}

\section{Intersection of N. Milwaukee Avenue with Desplaines/Kinzie}

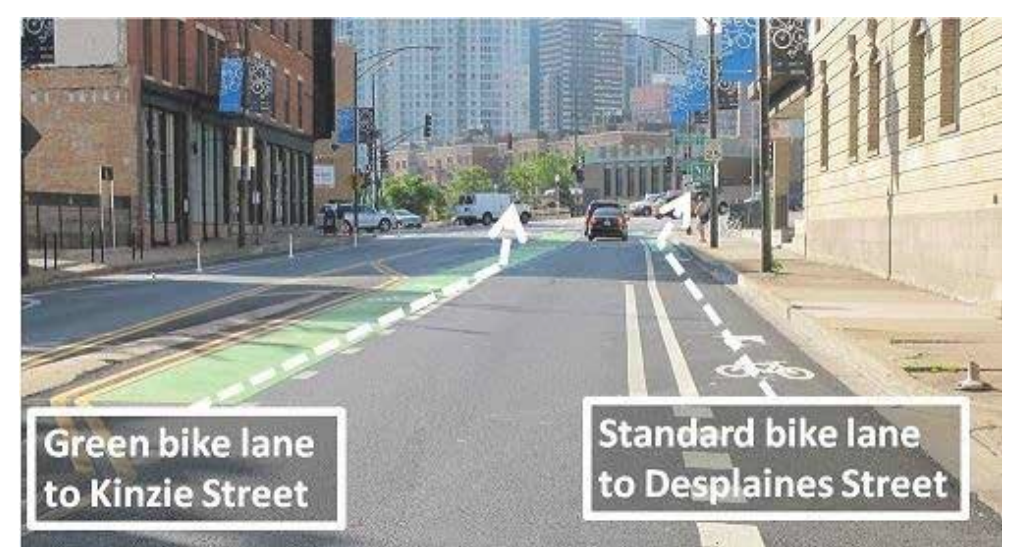

Consider the times you turn onto Kinzie from Milwaukee:

Because of the green lane to the left of the standard traffic lane on Milwaukee...

\begin{tabular}{|c|c|c|c|c|c|c|}
\hline & $\begin{array}{l}\text { Decreased a } \\
\text { Lot }\end{array}$ & $\begin{array}{l}\text { Decreased } \\
\text { Somewhat }\end{array}$ & $\begin{array}{c}\text { Not } \\
\text { Changed }\end{array}$ & $\begin{array}{l}\text { Increased } \\
\text { Somewhat }\end{array}$ & $\begin{array}{l}\text { Increased a } \\
\quad \text { Lot }\end{array}$ & No Opinion \\
\hline $\begin{array}{l}\text { how easy it is to merge across the standard traffic } \\
\text { lane (and into position to turn onto Kinzie) has ... }\end{array}$ & 0 & 0 & 0 & 0 & 0 & 0 \\
\hline $\begin{array}{l}\text { the amount of time I have to merge across the } \\
\text { standard traffic lane has ... }\end{array}$ & 0 & 0 & 0 & 0 & 0 & 0 \\
\hline $\begin{array}{r}\text { the amount of attention motorists pay to people on } \\
\text { bicycles has } \ldots\end{array}$ & 0 & 0 & 0 & 0 & 0 & 0 \\
\hline
\end{tabular}

Do you ride through this intersection differently now than you did before the changes were made?
Yes
No

How have you changed how you ride through the intersection? 
How comfortable do you feel when riding a bicycle through this intersection?

\begin{tabular}{|c|c|c|c|c|c|c|c|}
\hline & $\begin{array}{c}\text { (1) Very } \\
\text { Uncomfortable }\end{array}$ & (2) & (3) & (4) & (5) & $\begin{array}{l}\text { (6) Very } \\
\text { Comfortable }\end{array}$ & $\begin{array}{c}\text { Not } \\
\text { applicable }\end{array}$ \\
\hline $\begin{array}{l}\text { Turning left from N. Milwaukee Avenue onto Kinzie } \\
\text { Street }\end{array}$ & 0 & 0 & 0 & 0 & 0 & 0 & 0 \\
\hline $\begin{array}{l}\text { Turning right from N. Milwaukee Avenue onto } \\
\text { Desplaines Street }\end{array}$ & 0 & 0 & 0 & 0 & 0 & 0 & 0 \\
\hline
\end{tabular}

\section{Intersection of N. Milwaukee Avenue and N. Elston Avenue}

As you travel north on Milwaukee (out of downtown), a new bicycle signal has been installed at the intersection of Milwaukee Avenue and North Elston Avenue (see picture below). Previously, there was no bike signal and the bike lane was to the left of the right-turn lane.

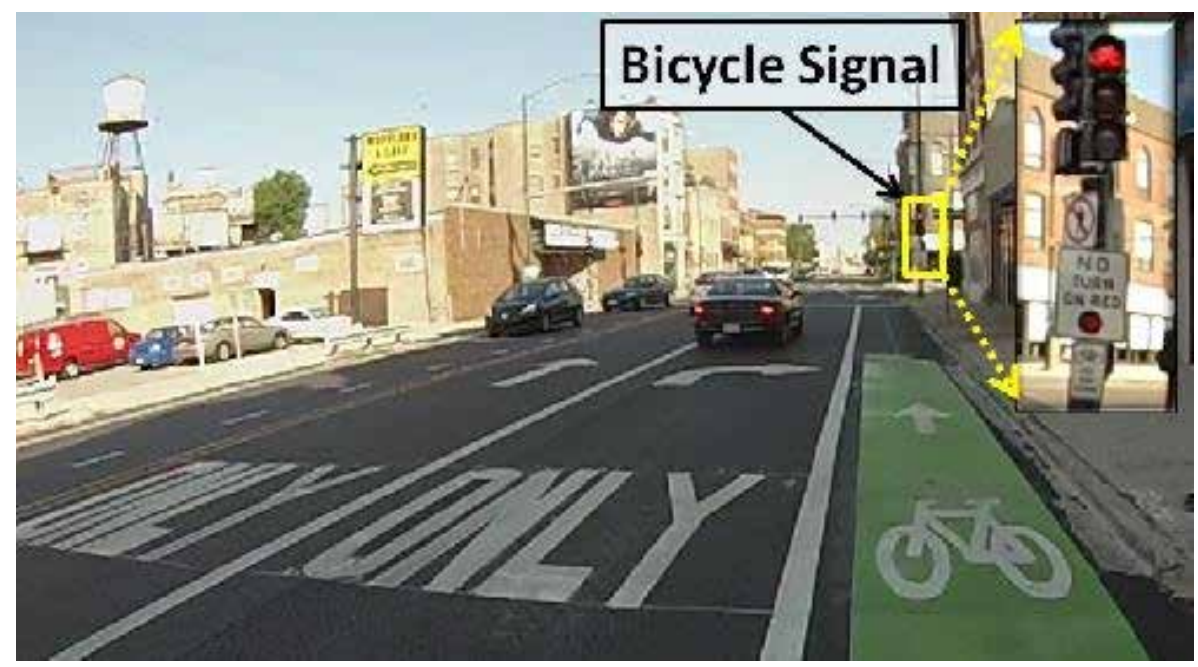

Have you bicycled through this intersection travelling north since the bicycle signal was installed?
Yes
No

When riding North through this intersection, do you most often:

Proceed straight through the intersection and continue on N. Milwaukee Avenue

Turn right onto N. Elston Avenue

When riding North through this intersection, do you most often:

Ride in the bike lane

Move out into the standard travel lane

Because of the changes to the intersection:

\begin{tabular}{|c|c|c|c|c|c|c|}
\hline & $\begin{array}{l}\text { Decreased a } \\
\text { Lot }\end{array}$ & $\begin{array}{l}\text { Decreased } \\
\text { Somewhat }\end{array}$ & Not Changed & $\begin{array}{l}\text { Increased } \\
\text { Somewhat }\end{array}$ & $\begin{array}{l}\text { Increased a } \\
\text { Lot }\end{array}$ & No Opinion \\
\hline $\begin{array}{l}\text { how safe I feel bicycling through this } \\
\text { intersection has... }\end{array}$ & 0 & 0 & 0 & 0 & 0 & 0 \\
\hline
\end{tabular}


how long I have to wait for a signal to get through the intersection has...

how likely I am wait for the signal to turn green before crossing has...

How comfortable do you feel bicycling through this intersection?

\begin{tabular}{|c|c|c|c|c|c|c|c|}
\hline & $\begin{array}{c}\text { (1) Very } \\
\text { Uncomfortable }\end{array}$ & (2) & (3) & (4) & (5) & $\begin{array}{l}\text { (6) Very } \\
\text { Comfortable }\end{array}$ & $\mathrm{N} / \mathrm{A}$ \\
\hline $\begin{array}{l}\text { When continuing STRAIGHT on } \\
\text { Milwaukee }\end{array}$ & 0 & 0 & 0 & 0 & 0 & 0 & 0 \\
\hline $\begin{array}{l}\text { When turning RIGHT onto } \\
\text { Elston }\end{array}$ & 0 & 0 & 0 & 0 & 0 & 0 & 0 \\
\hline
\end{tabular}

\section{Overall Impressions of the Protected Bike Lanes}

What is the best thing about the protected bike lanes?

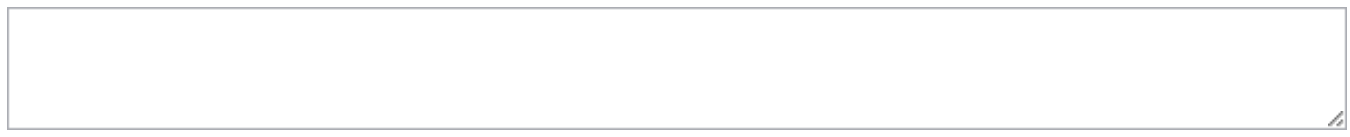

What should be improved about the protected bike lanes?

\section{About Bicycling and Comfort}

Regardless of whether you currently bicycle in all the following situations, please consider how comfortable you would be riding a bicycle in each place:

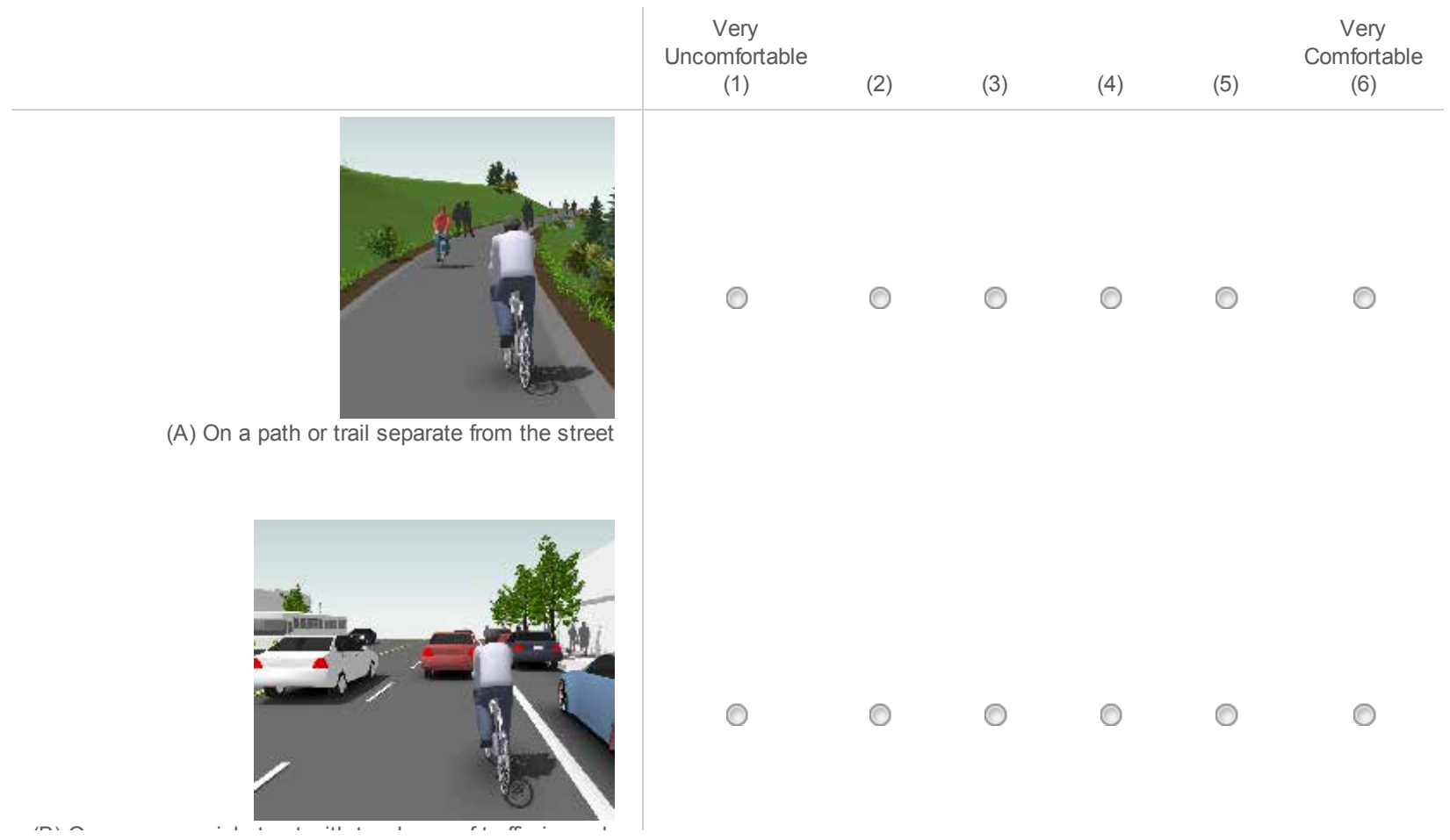


(B) Un a commercial street with two Ianes of trattic in eacn direction, with traffic speeds of 35 miles per hour, on-street car parking, and no bike lane

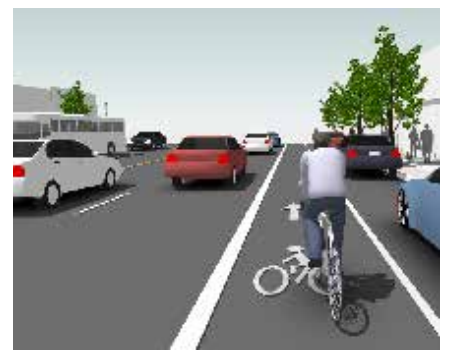

(C) On a similar street to (B), but with a striped bike lane added

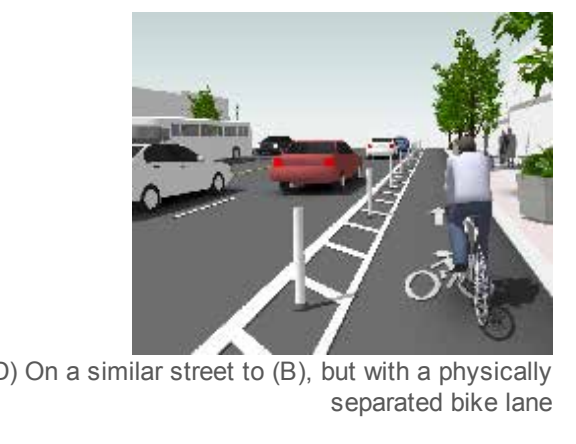

(D) On a similar street to (B), but with a physically separated bike lane
Very

Uncomfortable

(1)

$(2$

$(2)$

(3)

(4)

(5)

Very

comfortable

(6)

How comfortable would you feel bicycling on a commercial street with two lanes of traffic in each direction, with traffic speeds of 35 miles per hour (Situation D above), but with the following types of separation from traffic:
$\mid \begin{gathered}\text { Very } \\ \text { Uncomfortable }\end{gathered}$
(1)
(2)
(3)
(4)
(5)
Very Comfortable

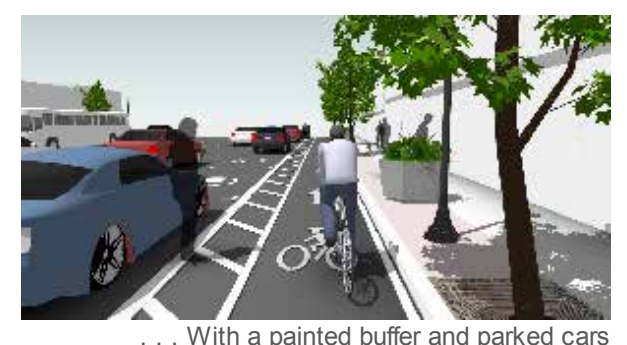

With a painted buffer and parked cars

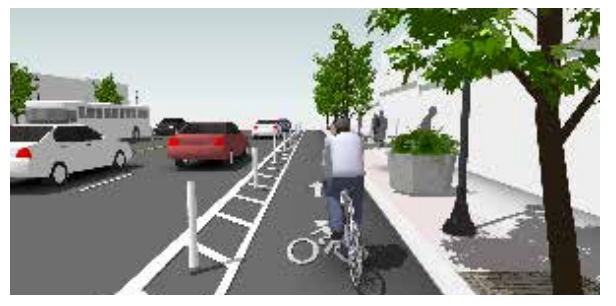




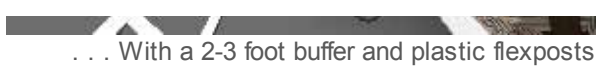

With a 2-3 foot buffer and plastic flexposts

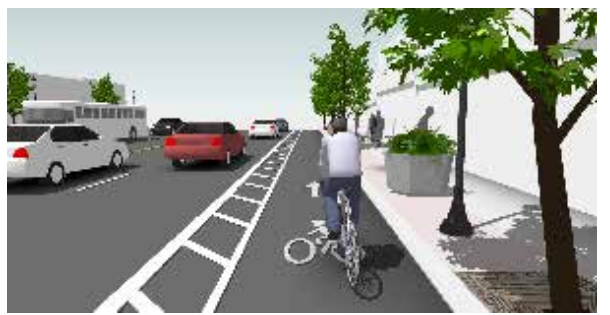

.. With a painted 2-3 foot buffer

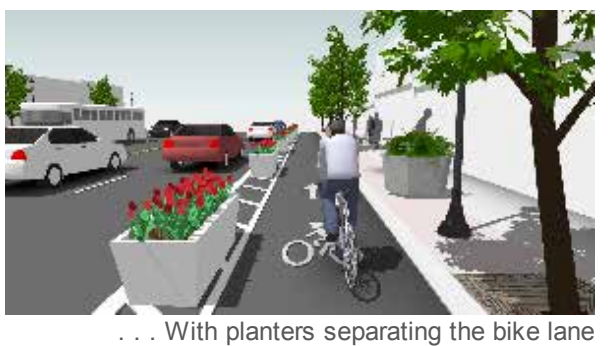

Very

Uncomfortable

(1)

(2)

(3)

(4)

(5)

Very

Comfortable

(6)

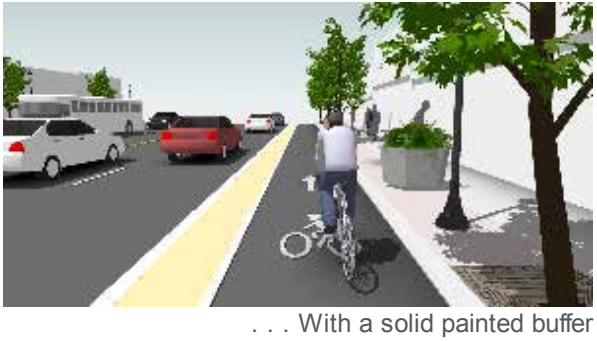

3.

\section{.}

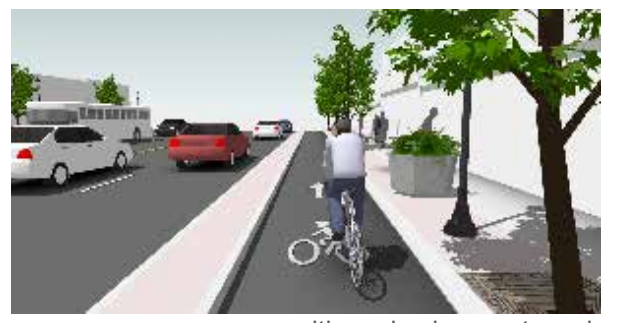

. with a raised concrete curb

○

\section{About People you Encounter on the Street}

Please indicate if you agree or disagree with the following statements:

\begin{tabular}{r|ccc} 
Somewhat & Strongly \\
Disagree & 0 & Somewhat Agree Strongly Agree & No Opinion \\
\hline Most drivers follow the rules of the road. & 0
\end{tabular}




\section{About You}

We have a few questions about you so that we may understand the characteristics of our survey respondents.

Do you have a current:

\begin{tabular}{l|cc} 
& Yes & \\
\hline Driver's License & $\bigcirc$ & $\bigcirc$ \\
Transit Pass & $\bigcirc$ \\
Diwy (Bike-share) Membership & $\bigcirc$ & $\bigcirc$ \\
Car-share Membership & 0
\end{tabular}

How many working motor vehicles does your household own or lease? (Do not include motorhomes).

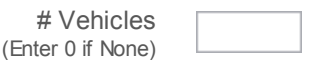

How many working adult bicycles does your household own?

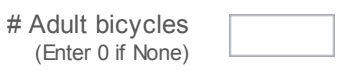

INCLUDING YOURSELF, how many people live in your household?

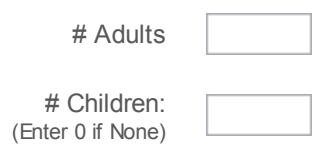

What is your home zip code?

5 digit zip code:

What is your gender?

Male

○
Female

○
O

What is your age? 


\section{Years}

Do you consider yourself: (select all that apply)

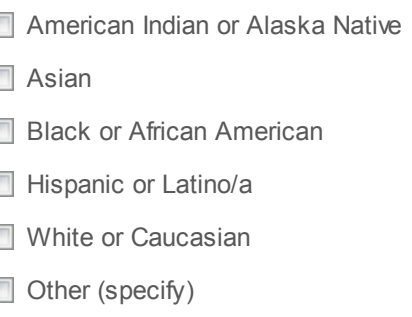

What is your employment status? (Select all that apply)

$\square$ I work outside the home -> What is the zip code of your place of work?

I go to school outside the home -> What is the zip code of your school?

I work from home

$\square$ Not employed at this time

What is your annual household income?
Less than $\$ 25,000$
$\$ 25,000$ to less than $\$ 50,000$
$\$ 50,000$ to less than $\$ 75,000$
$\$ 75,000$ to less than $\$ 100,000$
- $\$ 100,000$ to less than $\$ 200,000$
$\$ 200,000$ or more
I prefer not to provide this information

How many years of school have you completed?
Some high school or less
High school diploma or GED
Some College
Trade/Vocational School
Associate Degree
Four-year college degree or more
Other (please specify):

Is there anything else that you would like to tell us? 
That's all the questions we have. Would you like to be entered into a drawing for one of three $\$ 100$ gift cards to Amazon.com?

(2) Yes, I would like to be entered in the drawing ('Ill enter my name on the next page).

No thanks. I will complete my survey by clicking the ">>" button

Enter your name and some way for us to get a hold of you below. We will only use this information to contact you in the event that your entry is selected to receive a gift card. This information will not be associated to any of your survey responses.

Name

Phone or email

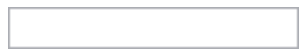

Thanks for taking the survey - please make sure to click the ">>" button to submit your entry and close the survey! 


\section{BiCYCLIST: CHICAGo - DEARBoRn AVE.}




\section{Neighborhood Street Study}

Default Question Block

Dear Bicyclist,

My name is Chris Monsere and I am a faculty member at Portland State University in the Department of Civil and Environmental Engineering. I am the principal investigator on a project researching separated bicycle lanes in six cities across the country.

Hearing from bicyclists like yourself is a very important part of this study. We are only sampling a select number of bicyclists near the Dearborn Street protected bike lanes. Therefore, every response is very important and we hope you will participate. We will share our findings with the Chicago Department of Transportation and hope that the results will help in future plans for improving bicycling in cities around the United States.

The survey, which starts on the next page, should take about 10-20 minutes. Your participation in the study is voluntary and you may stop taking the survey at any time with no consequences.

The postcard that you received has a unique number that only identifies where and when we handed you the postcard. We will protect the confidentiality of your individual survey responses. None of your responses will be linked to your name or other identifying personal information.

To say thank you, all people who complete their survey by 10/10/13 will be entered into a drawing for one of three $\$ 100$ Amazon.com gift cards. You will be asked to enter your name and a way to contact you at the end of the survey - this information is voluntary and will not be connected to your survey data.

If you have concerns or problems about your participation in this study or your rights as a research subject, please contact the Human Subjects Research Review Committee, Office of Research and Strategic Partnerships, Market Center Building Suite 620, Portland State University, (877480-4400). If you have questions about the study itself, please contact our research team directly at streets@pdx.edu or 503-725-2875.

Sincerely,

Christopher M. Monsere, Ph.D., P.E., Associate Professor \& Associate Chair Civil and Environmental Engineering Portland State University

Do you agree to participate in this survey?
No
Yes

When you received the postcard for this survey, where were you ...

\begin{tabular}{c|c|ccc|c|c|} 
Can you provide an address or \\
closest intersection to this place?
\end{tabular}

If you selected other, please explain: 
What type of bicycle were you riding when you received the postcard?

Personal Bicycle

Dimy (Bike Share) Bicycle

Rental Bicycle

Other (please specify):

\section{Dearborn Street Protected Bike Lanes}

A two-way protected bikeway was recently built on Dearborn Street between West Kinzie Street and West Polk Street. Bike lanes were separated from other traffic lanes by a combination of a striped "buffer" zone, plastic "flexposts" and by moving parked cars away from the curb to provided a protected lane (see picture below). Bicycle signals and left-turn signals were added at many intersections as well.
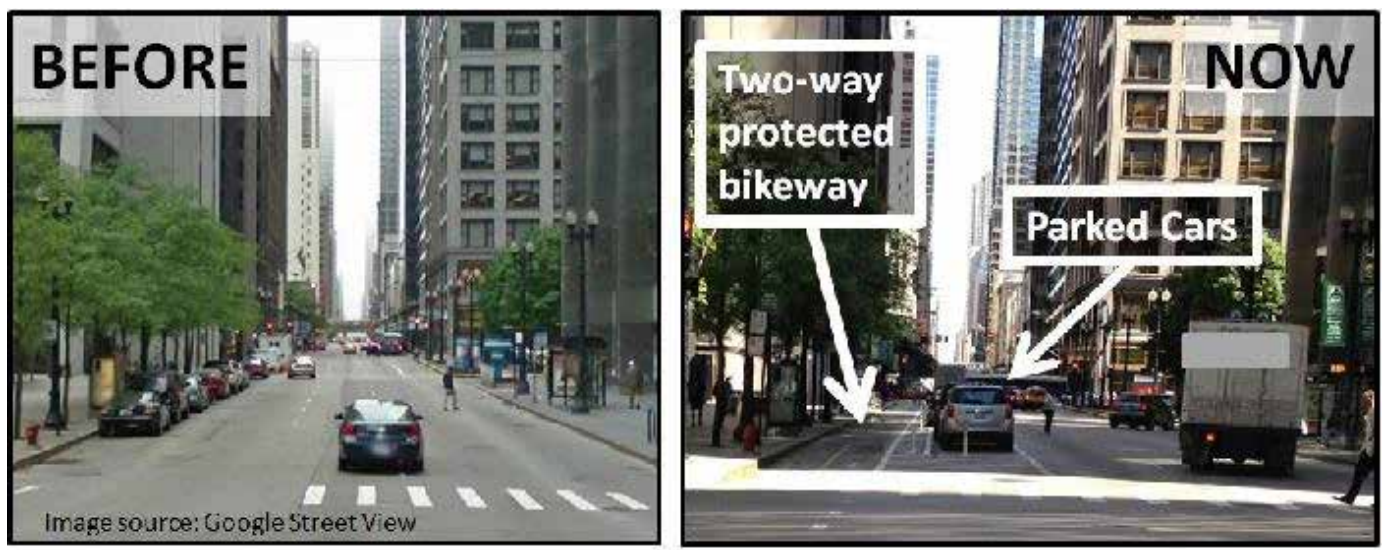

Extent of Dearborn Street protected bike lanes: 


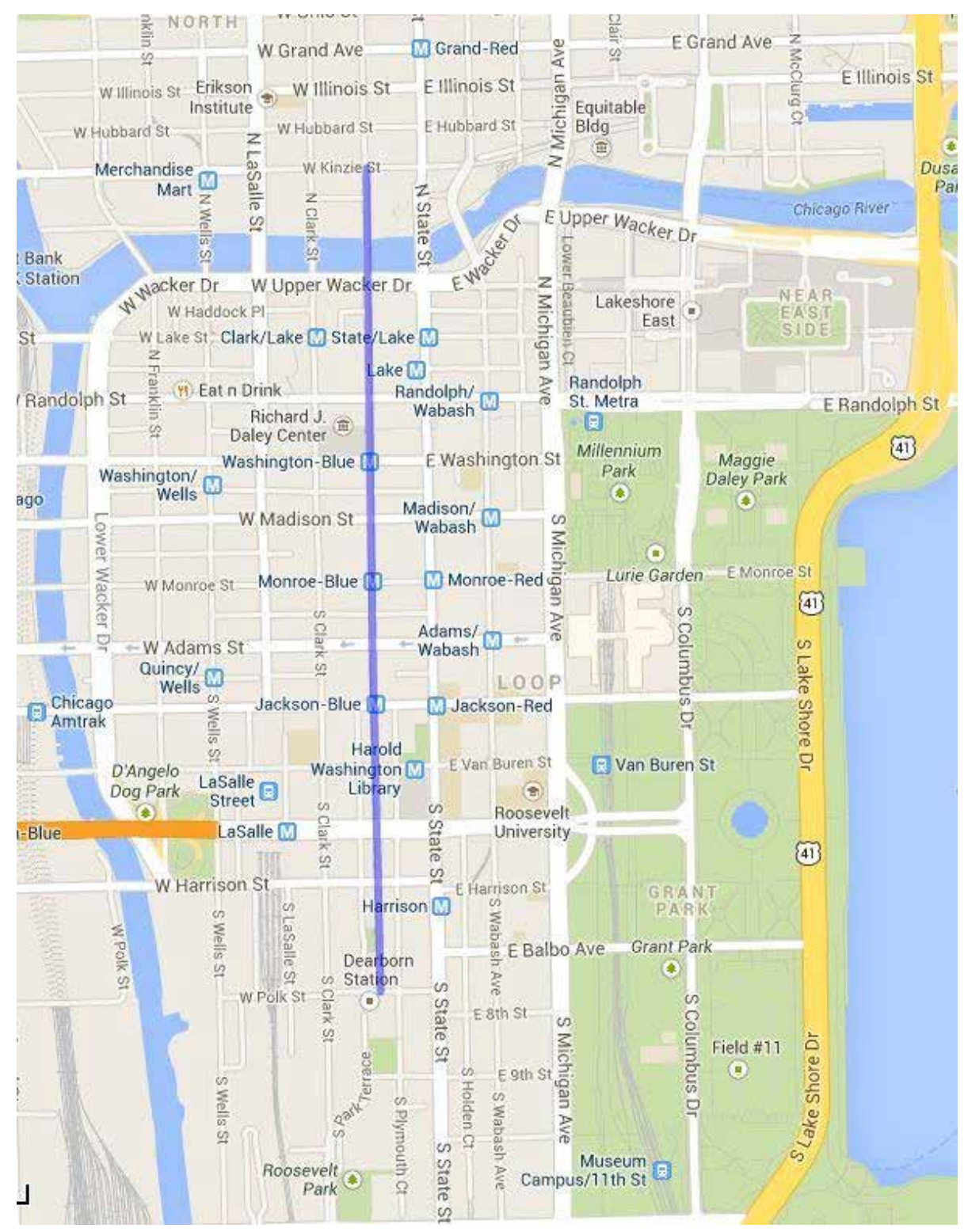

How often do you ride a bicycle on this part of Dearborn Street?
This was my first time
Less than once a month
A few times a month
A few times a week
Daily

How comfortable do you feel when bicycling SOUTHBOUND in the Dearborn Street protected bike lanes (in the lane closest to the sidewalk)? 

$\checkmark$ (1) very viluullivilanic
(2)
(3)
(4)
(5)
(6) Very Comfortable

How comfortable do you feel when bicycling NORTHBOUND in the Dearborn Street protected bike lanes (in the lane closest to the standard traffic lanes and plastic flexposts)?
(1) Very Uncomfortable
(2)
(3)
(4)
(5)
(6) Very Comfortable

Since the Dearborn Street protected bike lanes were built, do you travel on this route:
Less frequently
More frequently
About the same
This is my first time on this route

When bicycling on Dearborn Street between Kinzie and Polk, how often do you ride on the following:

\begin{tabular}{|c|c|c|c|c|}
\hline & Always & Most of the time & Some of the time & Never \\
\hline Protected bike lanes & 0 & 0 & 0 & 0 \\
\hline Standard traffic lanes & 0 & 0 & 0 & 0 \\
\hline Curb Area & 0 & 0 & 0 & 0 \\
\hline Sidewalk & 0 & 0 & 0 & 0 \\
\hline
\end{tabular}

Consider the trip you were making when you were handed the postcard. Before the Dearborn Street protected bike lanes were built, how would you have made this trip?
By bicycle, using this same route
By bicycle, using another route - (Please specify which route):
By foot
By public transportation
By car
By taxi
I would NOT have taken this trip
Other (please specify below)

\section{About Changes due to the Protected Bike Lanes}

Because of the Dearborn Street protected bike lanes... 


\begin{tabular}{|c|c|c|c|c|c|c|}
\hline & $\begin{array}{l}\text { Decreased } \\
\quad \text { a Lot }\end{array}$ & $\begin{array}{l}\text { Decreased } \\
\text { Somewhat }\end{array}$ & $\begin{array}{c}\text { Not } \\
\text { Changed }\end{array}$ & $\begin{array}{l}\text { Increased } \\
\text { Somewhat }\end{array}$ & $\begin{array}{l}\text { Increased } \\
\quad \text { a Lot }\end{array}$ & $\begin{array}{l}\text { No } \\
\text { Opinion }\end{array}$ \\
\hline I feel the safety of bicycling on Dearborn Street has. & 0 & 0 & 0 & 0 & 0 & 0 \\
\hline the time it takes me to bicycle on Dearborn Street has . & 0 & 0 & 0 & ○ & 0 & 0 \\
\hline the usefulness of Dearborn Street for getting to places I want to go has & 0 & 0 & 0 & 0 & O & 0 \\
\hline how often I stop at shops and businesses on Dearborn Street has ... & O & 0 & 0 & 0 & O & 0 \\
\hline how comfortable I feel when bicycling on Dearborn Street has. & 0 & 0 & 0 & 0 & 0 & 0 \\
\hline drivers' awareness of people biking on Dearborn Street has & ○ & ○ & O & O & O & ○ \\
\hline drivers' speeds on Dearborn Street have. & ○ & O & ○ & ○ & ○ & 0 \\
\hline how often I ride a bicycle overall has. & O & 0 & O & ○ & O & O \\
\hline the difficulty of navigating around turning motor vehicles has ... & 0 & ○ & O & ○ & O & 0 \\
\hline the difficulty of navigating around pedestrians has ... & 0 & 0 & 0 & 0 & 0 & 0 \\
\hline
\end{tabular}

\section{Your Opinions about the Protected Bike Lanes}

Please indicate if you agree or disagree with the following statements about the Dearborn Street protected bike lanes:

\begin{tabular}{|c|c|c|c|c|c|}
\hline & $\begin{array}{l}\text { Strongly } \\
\text { Disagree }\end{array}$ & $\begin{array}{l}\text { Somewhat } \\
\text { Disagree }\end{array}$ & $\begin{array}{l}\text { Somewhat } \\
\text { Agree }\end{array}$ & $\begin{array}{l}\text { Strongly } \\
\text { Agree }\end{array}$ & No Opinion \\
\hline $\begin{array}{l}\text { I would go out of my way to ride on Dearborn Street } \\
\text { compared to other streets. }\end{array}$ & 0 & 0 & 0 & 0 & 0 \\
\hline $\begin{array}{c}\text { When bicycling SOUTHBOUND, I prefer the protected } \\
\text { bike lanes on Dearbon Street to riding south on Clark } \\
\text { Street. }\end{array}$ & 0 & 0 & 0 & 0 & 0 \\
\hline $\begin{array}{r}\text { Taking Dearborn Street is the most direct bicycle route to } \\
\text { my destination. }\end{array}$ & 0 & 0 & 0 & 0 & 0 \\
\hline $\begin{array}{l}\text { The } \frac{\text { buffer section with parked cars between the traffic }}{\text { lanes and the bike lane makes me feel safe. }}\end{array}$ & 0 & 0 & 0 & 0 & 0 \\
\hline \multirow[t]{2}{*}{$\begin{array}{l}\text { The buffer section with plastic flexposts between the } \\
\text { traffic lanes and the bike lane makes me feel safe. }\end{array}$} & 0 & 0 & 0 & 0 & 0 \\
\hline & $\begin{array}{l}\text { Strongly } \\
\text { Disagree }\end{array}$ & $\begin{array}{l}\text { Somewhat } \\
\text { Disagree }\end{array}$ & $\begin{array}{l}\text { Somewhat } \\
\text { Agree }\end{array}$ & $\begin{array}{l}\text { Strongly } \\
\text { Agree }\end{array}$ & No Opinion \\
\hline $\begin{array}{r}\text { During the winter, snow is quickly removed from the } \\
\text { protected bike lanes. }\end{array}$ & 0 & 0 & 0 & 0 & 0 \\
\hline $\begin{array}{l}\text { Leaves and debris in the protected bike lanes are worse } \\
\text { than other places I ride. }\end{array}$ & 0 & 0 & 0 & 0 & 0 \\
\hline $\begin{array}{l}\text { Puddles and standing water are worse than other places I } \\
\text { ride. }\end{array}$ & 0 & 0 & 0 & 0 & 0 \\
\hline $\begin{array}{l}\text { The protected bike lanes are wide enough for me to ride } \\
\text { comfortably. }\end{array}$ & 0 & 0 & 0 & 0 & 0 \\
\hline \multirow[t]{2}{*}{$\begin{array}{l}\text { The protected bike lanes are wide enough for one } \\
\text { bicyclist to pass another in the same direction. }\end{array}$} & 0 & 0 & 0 & 0 & 0 \\
\hline & $\begin{array}{l}\text { Strongly } \\
\text { Disagree }\end{array}$ & $\begin{array}{l}\text { Somewhat } \\
\text { Disagree }\end{array}$ & $\begin{array}{l}\text { Somewhat } \\
\text { Agree }\end{array}$ & $\begin{array}{l}\text { Strongly } \\
\text { Agree }\end{array}$ & No Opinion \\
\hline $\begin{array}{l}\text { The protected bike lanes are wide enough for two people } \\
\text { to comfortably ride side-by-side in the same direction. }\end{array}$ & 0 & 0 & 0 & 0 & 0 \\
\hline $\begin{array}{r}\text { If I am bicycling with another adult, I would prefer to ride } \\
\text { side-by-side. }\end{array}$ & 0 & 0 & 0 & 0 & 0 \\
\hline $\begin{array}{r}\text { The protected bike lanes are safer than other bike lanes } \\
\text { in Chicago. }\end{array}$ & 0 & 0 & 0 & 0 & $\bigcirc$ \\
\hline $\begin{array}{l}\text { During rush hour the bike lanes get congested with } \\
\text { bicyclists. }\end{array}$ & 0 & 0 & 0 & 0 & 0 \\
\hline
\end{tabular}


Please indicate if you disagree or agree with the following statements about the effectiveness of the Dearborn Street protected bike lanes:

$\begin{gathered}\text { Strongly } \\ \text { Disagree } \\ \text { and where the designated bicycle lanes are. }\end{gathered}$
$\begin{gathered}\text { The intersection signals, signs, and street markings } \\ \text { Disagree }\end{gathered}$
make it clear who has the right-of-way at intersections.
$\begin{gathered}\text { Motorists know not to turn across the bike lanes at } \\ \text { intersection when the bicycle signal is green (and the } \\ \text { turn arrow for cars is red) }\end{gathered}$
$\begin{gathered}\text { The buffer effectively separates bikes from cars. } \\ \text { The buffer does a good job at protecting bikes from cars. } \\ \text { The protected bike lanes make drivers and bicyclists } \\ \text { more predictable. }\end{gathered}$
The protected bike lanes make it clear where bicyclists
and pedestrians should be.

What do you think it means when the pavement is painted green along the bikeway?

\section{About What You Encounter in the Protected Bike Lanes}

Please indicate how often you have observed the following on your rides in the Dearborn Street protected bike lanes, AND whether you think this is a problem that needs to be addressed

\begin{tabular}{|c|c|c|c|c|c|c|c|}
\hline & \multicolumn{4}{|c|}{$\begin{array}{l}\text { How often do you encounter the following } \\
\text { IN the Protected Bike Lanes? }\end{array}$} & \multicolumn{3}{|c|}{ How much of a problem is this? } \\
\hline & Never & Rarely & Sometimes & Often & $\begin{array}{l}\text { Not a } \\
\text { problem }\end{array}$ & $\begin{array}{l}\text { Minor } \\
\text { problem }\end{array}$ & $\begin{array}{l}\text { Major } \\
\text { Problem }\end{array}$ \\
\hline Cars parking & 0 & 0 & 0 & 0 & 0 & 0 & 0 \\
\hline $\begin{array}{l}\text { Cars loading or } \\
\text { unloading passengers }\end{array}$ & 0 & 0 & 0 & 0 & 0 & 0 & 0 \\
\hline $\begin{array}{l}\text { Delivery vehicles } \\
\text { loading or unloading }\end{array}$ & 0 & 0 & 0 & 0 & 0 & 0 & 0 \\
\hline Taxis & 0 & 0 & 0 & 0 & 0 & 0 & 0 \\
\hline $\begin{array}{r}\text { Cars/trucks driving } \\
\text { where they are not } \\
\text { supposed to (in the } \\
\text { bike lanes) }\end{array}$ & 0 & 0 & 0 & 0 & 0 & 0 & 0 \\
\hline $\begin{array}{l}\text { Cars/trucks waiting to } \\
\text { make turns OFF of } \\
\text { Dearborn Street }\end{array}$ & 0 & 0 & 0 & 0 & ○ & 0 & 0 \\
\hline $\begin{array}{r}\text { Cars/trucks waiting to } \\
\text { pull out ONTO } \\
\text { Dearborn Street }\end{array}$ & 0 & 0 & 0 & 0 & 0 & 0 & 0 \\
\hline $\begin{array}{r}\text { People walking in the } \\
\text { bike lanes }\end{array}$ & 0 & 0 & 0 & 0 & 0 & 0 & 0 \\
\hline $\begin{array}{r}\text { People standing in } \\
\text { the bike lanes while } \\
\text { waiting to cross the } \\
\text { street }\end{array}$ & 0 & 0 & 0 & 0 & 0 & 0 & 0 \\
\hline
\end{tabular}



$\square$ Yes, a collision
Yes, a near-collision
No, neither

If you have been involved in a collision or near-collision, please indicate what other people or objects were involved.

Check all that apply.

\begin{tabular}{|c|c|c|}
\hline & Collision & Near Collision \\
\hline Another bicyclist & $\square$ & $\square$ \\
\hline A pedestrian & $\square$ & $\square$ \\
\hline A turning car & $\square$ & $\square$ \\
\hline A parking car & $\square$ & $\square$ \\
\hline A parked car & $\square$ & $\square$ \\
\hline A delivery truck & $\square$ & $\square$ \\
\hline \multicolumn{3}{|l|}{ A bus } \\
\hline & $\square$ & $\square$ \\
\hline A taxi & $\square$ & $\square$ \\
\hline One of the plastic flexposts & $\square$ & $\square$ \\
\hline $\begin{array}{l}\text { Other stationary object (please } \\
\text { describe). }\end{array}$ & $\square$ & $\square$ \\
\hline $\begin{array}{r}\text { Something else (please } \\
\text { describe). }\end{array}$ & $\square$ & $\square$ \\
\hline
\end{tabular}

Please provide a brief description of the collision(s) you were involved in while riding a bicycle in the protected bike lanes.

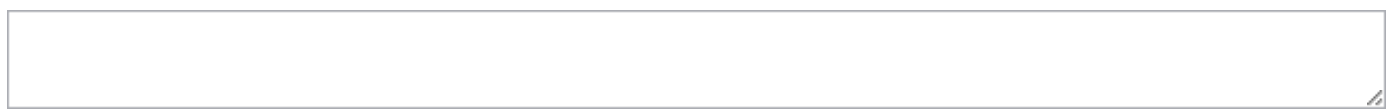

\section{$\underline{\text { About Intersections }}$}

At many intersections along Dearborn Street, new bicycle signals were added, along with a left-turn signal for motor vehicles in the left-turn lane (see picture). 


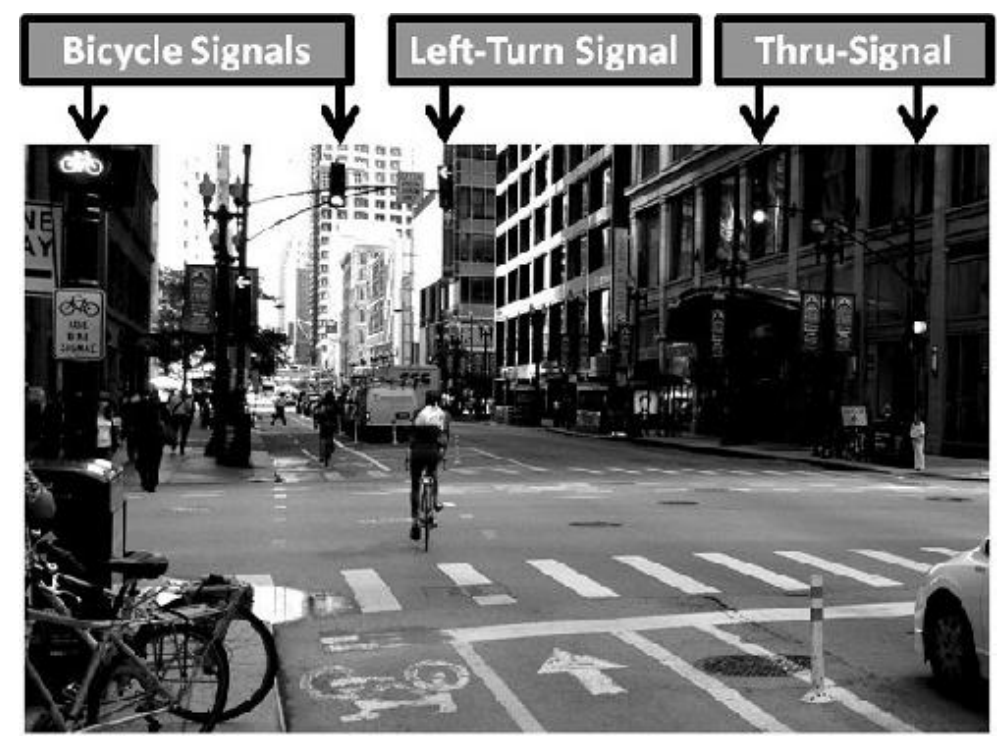

How comfortable do you feel bicycling through these intersections on Dearborn Street?

\begin{tabular}{l|cccccc} 
& $\begin{array}{c}(1) \text { Very } \\
\text { Uncomfortable }\end{array}$ & $(2)$ & $(3)$ & $(4)$ & (5) & $\begin{array}{c}(6) \text { Very } \\
\text { Comfortable }\end{array}$ \\
\hline When traveling SOUTHBOUND & 0 & 0 & 0 & 0 & 0 & 0 \\
When traveling NORTHBOUND & 0 & 0 & 0 & $\bigcirc$ & 0 & 0
\end{tabular}

Please indicate if you agree or disagree with the following statements:

The bicycle signal stays green long enough for me to ride
through the intersection comfortably.
Motorists generally understand to folllow left turn signals
at these intersections.
I often see motorists making left turns when the bicycle
signal is green (and the left turn signal is red).
I generally feel safe when bicycling through the
intersection.
I often encounter pedestrians waiting in the bike lanes
when the bicycle signal is green.
Its generally not worth my time to wait for the bicycle
signal to turn green on Dearborn Street.

How often are the following blocking your pathway through the intersection when the bicycle signal is green:

\begin{tabular}{l|cccc} 
& Never & Some of the time & Most of the time & Always \\
\hline Cars/trucks & 0 & 0 & 0 & 0 \\
Pedestrians & 0 & 0 & 0 & 0
\end{tabular}


the protectea bıke Ianes ana across tne trattıc Ianes (see pıcture)

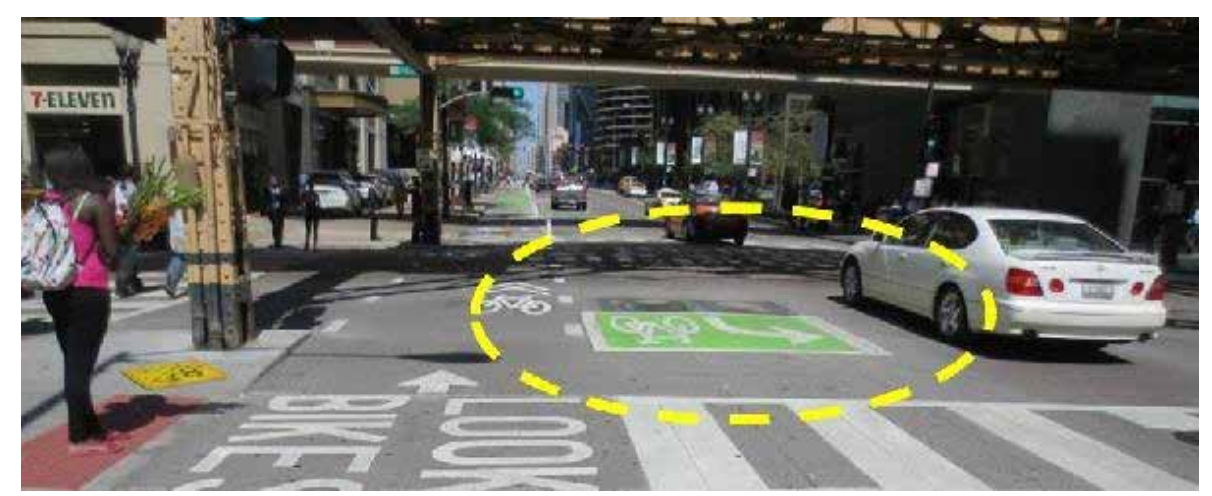

Have you ever waited to cross Dearborn in one of these green boxes when bicycling?

Yes, I have

No - I've never had the opportunity

No - l've chosen not to wait in one of these boxes

Compared to crossing Dearborn WITHOUT a green queue box, waiting in this green box is...
A lot LESS convenient
Somehwat LESS convenient
No Difference
Somewhat MORE convenient
$\bigcirc$ A lot MORE convenient

Compared to crossing Dearborn WITHOUT a green queue box, waiting in this green box makes me feel ...
A lot LESS comfortable
Somehwat LESS comfortable
No Difference
Somewhat MORE comfortable
A lot MORE comfortable

\section{Overall Impressions of the Protected Bike Lanes}

What is the best thing about the protected bike lanes?

What should be improved about the protected bike lanes?

\section{About Bicycling and Comfort}


Regardless of whether you currently bicycle in all the following situations, please consider how comtortable you would be riding a bicycle in each place:

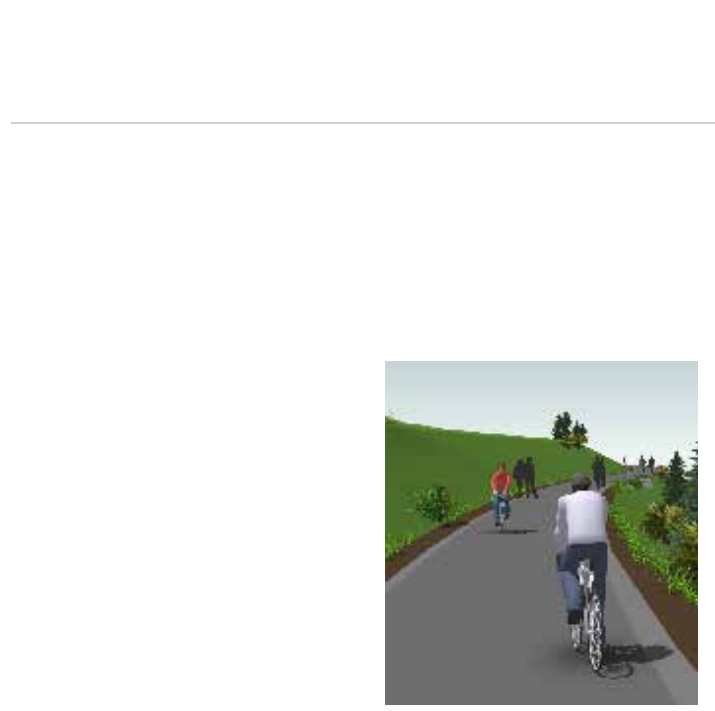

(A) On a path or trail separate from the street

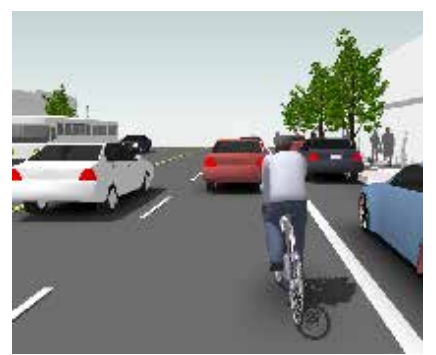

(B) On a commercial street with two lanes of traffic in each direction, with traffic speeds of 35 miles per hour, on-street car parking, and no bike lane

(C) On a similar street to (B), but with a striped bike lane
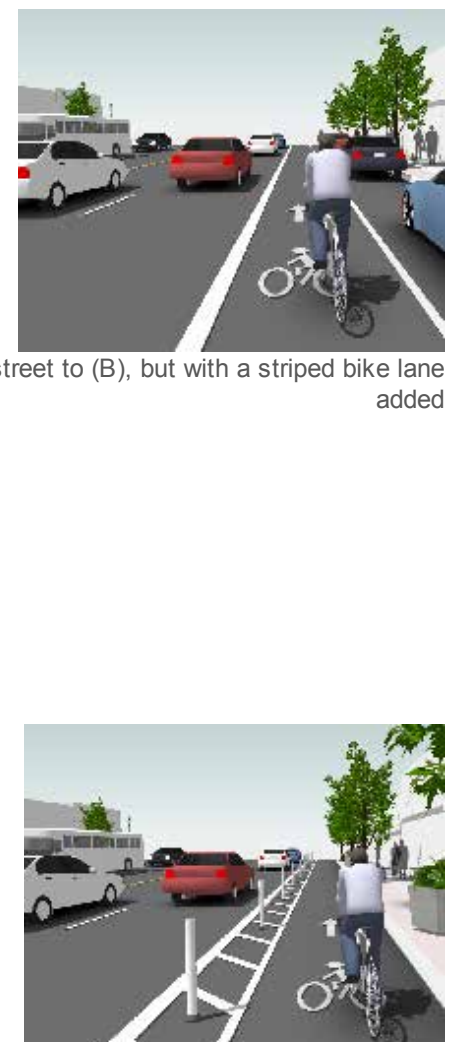

Very

Uncomfortable

(1)

(2)

(3)

(4)

(5)

comfortable

(6)

Very Comfortable

comfortable

(1)

(2)

(3)

(4)

(5)

(6) 
(D) On a similar street to (B), but with a physically

How comfortable would you feel bicycling on a commercial street with two lanes of traffic in each direction, with traffic speeds of 35 miles per hour (Situation D above), but with the following types of separation from traffic:

Very

Uncomfortable

(1)
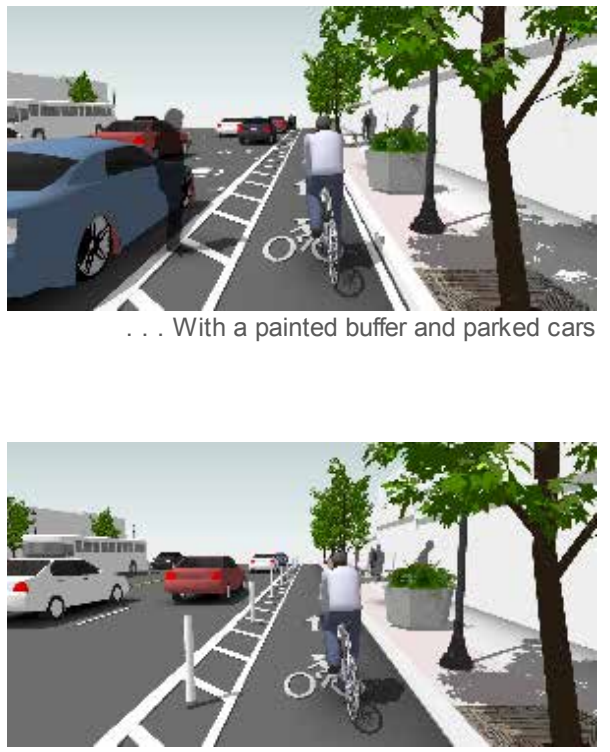

... With a 2-3 foot buffer and plastic flexposts
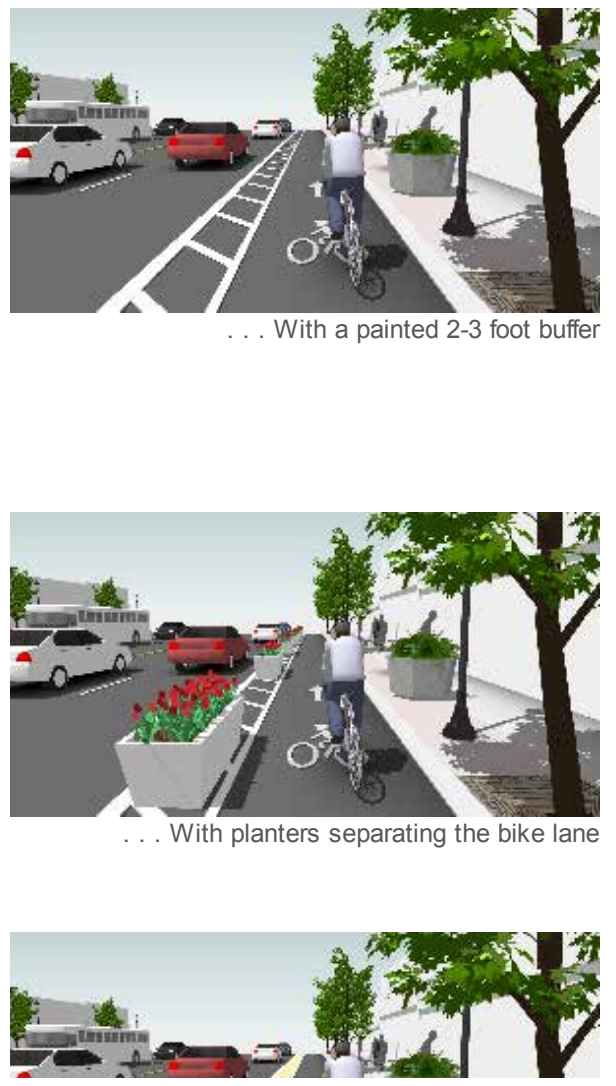

Very Uncomfortable

(1)
Very

Comfortable
(6)
(2)

(3)

(4)

(5)

-



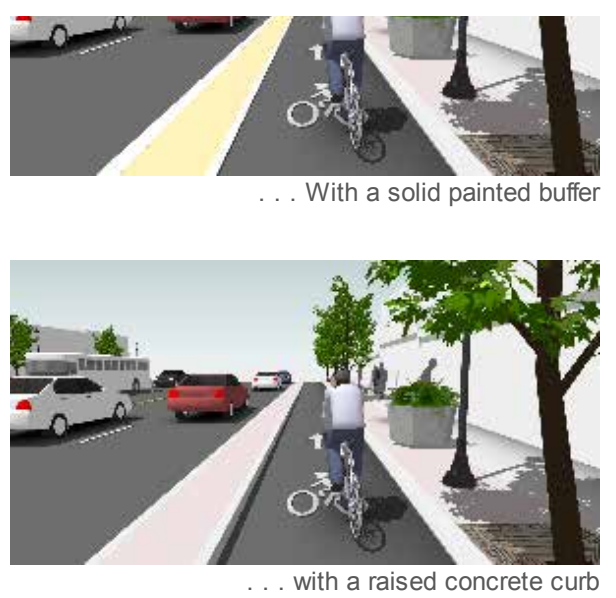

\section{About People you Encounter on the Street}

Please indicate if you agree or disagree with the following statements:

\begin{tabular}{|c|c|c|c|c|c|}
\hline & $\begin{array}{l}\text { Strongly } \\
\text { Disagree }\end{array}$ & $\begin{array}{l}\text { Somewhat } \\
\text { Disagree }\end{array}$ & Somewhat Agree & Strongly Agree & No Opinion \\
\hline Most drivers follow the rules of the road. & 0 & 0 & 0 & 0 & 0 \\
\hline Most drivers are predictable. & 0 & 0 & 0 & 0 & 0 \\
\hline Most bicyclists follow the rules of the road. & 0 & 0 & 0 & 0 & 0 \\
\hline Most bicyclists are predictable. & 0 & 0 & 0 & 0 & 0 \\
\hline Most pedestrians follow the rules of the road. & 0 & 0 & 0 & 0 & 0 \\
\hline Most pedestrians are predictable. & 0 & 0 & 0 & 0 & 0 \\
\hline
\end{tabular}

\section{About You}

We have a few questions about you so that we may understand the characteristics of our survey respondents.

Do you have a current:

\begin{tabular}{l|cc} 
& Yes & No \\
Driver's License & $\odot$ & $\bigcirc$ \\
Transit Pass & $\bigcirc$ & $\bigcirc$ \\
Diwy (Bike-share) Membership & $\bigcirc$ & $\bigcirc$ \\
Car-share Membership & $\odot$ & $\bigcirc$
\end{tabular}

How many working motor vehicles does your household own or lease? (Do not include motorhomes).

\footnotetext{
\# Vehicles (Enter 0 if None)
} 
How many working adult bicycles does your household own?

\# Adult bicycles (Enter 0 if None)

INCLUDING YOURSELF, how many people live in your household?

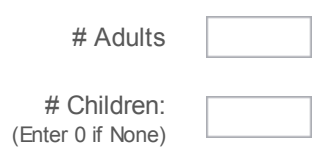

What is your home zip code?

5 digit zip code:

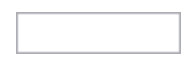

What is your gender?

Male

○
Female

O

What is your age?

Years

Do you consider yourself: (select all that apply)

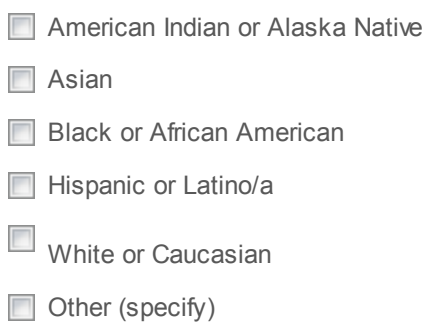

What is your employment status? (Select all that apply)

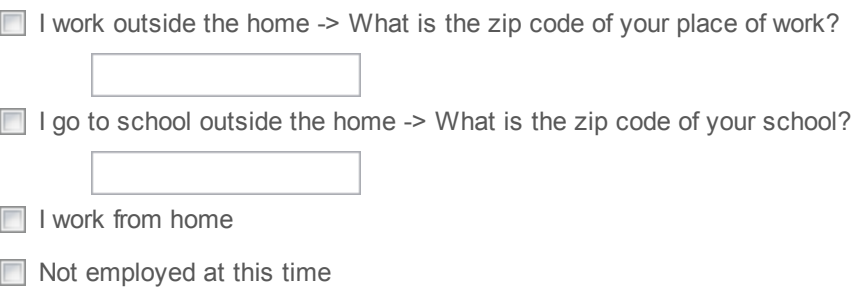

What is your annual household income? 
Less than $\$ 25,000$

$\$ 25,000$ to less than $\$ 50,000$

$\$ 50,000$ to less than $\$ 75,000$

$\$ 75,000$ to less than $\$ 100,000$

$\$ 100,000$ to less than $\$ 200,000$

$\$ 200,000$ or more

I prefer not to provide this information

How many years of school have you completed?
Some high school or less
High school diploma or GED
Some College
Trade/Vocational School
Associate Degree
Four-year college degree or more
Other (please specify):

Is there anything else that you would like to tell us?

That's all the questions we have. Would you like to be entered into a drawing for one of three $\$ 100$ gift cards to Amazon.com?

(2) Yes, I would like to be entered in the drawing ('IIl enter my name on the next page).

No thanks. I will complete my survey by clicking the ">>" button

Enter your name and some way for us to get a hold of you below. We will only use this information to contact you in the event that your entry is selected to receive a gift card. This information will not be associated to any of your survey responses.

Name

Phone or email

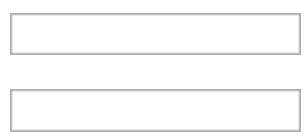

Thanks for taking the survey - please make sure to click the ">>" button to submit your entry and close the survey! 


\section{BiCYCLIST: PORTLAND - NE MULTNOMAH ST.}




\section{Neighborhood Street Study}

Default Question Block

Dear Bicyclist,

My name is Chris Monsere and I am a faculty member at Portland State University in the Department of Civil and Environmental Engineering. I am the principal investigator on a project researching protected bikeways (also called "cycle tracks") in six cities across the country.

Hearing from bicyclists like yourself is a very important part of this study. We are only sampling a select number of bicyclists near the NE Multnomah Street protected bikeway. Therefore, every response is very important and we hope you will participate. We will share our findings with the Portland Bureau of Transportation and hope that the results will help in future plans for improving bicycling in cities around the United States.

The survey, which starts on the next page, should take about 10-20 minutes. Your participation in the study is voluntary and you may stop taking the survey at any time with no consequences

The postcard that you received has a unique number that only identifies where and when we handed you the postcard. We will protect the confidentiality of your individual survey response. None of your responses will be linked to your name or other identifying personal information .

To say thank you, all people who complete their survey by October 17, 2013 will be entered into a drawing for one of three $\$ 100$ Amazon.com gift cards. You will be asked to enter your name and a way to contact you at the end of the survey - this information is voluntary and will not be connected to your survey data.

If you have concerns or problems about your participation in this study or your rights as a research subject, please contact the Human Subjects Research Review Committee, Office of Research and Strategic Partnerships, Market Center Building Suite 620, Portland State University, (877480-4400). If you have questions about the study itself, please contact our research team directly at streets@pdx.edu or 503-725-2875.

Sincerely,

Christopher M. Monsere, Ph.D., P.E.,

Associate Professor \& Associate Chair

Civil and Environmental Engineering

Portland State University

Do you agree to participate in this survey?
No
Yes

When you received the postcard for this survey, where were you ...

\begin{tabular}{|c|c|c|c|c|c|c|c|}
\hline & & & & Place & & & $\begin{array}{l}\text { Can you provide an address or } \\
\text { closest intersection to this place? }\end{array}$ \\
\hline & Home & Work & School & $\begin{array}{c}\text { Shopping / } \\
\text { Errands }\end{array}$ & $\begin{array}{l}\text { Just out } \\
\text { riding }\end{array}$ & Other & Address/Intersection \\
\hline . . . coming FROM on your bicycle? & 0 & 0 & 0 & 0 & 0 & 0 & \\
\hline . . . going TO on your bicycle? & 0 & 0 & 0 & 0 & 0 & 0 & \\
\hline
\end{tabular}

If you selected other, please explain: 


\section{NE Multnomah Protected Bikeway}

A protected bikeway, also known as a "cycle track," was recently built on NE Multnomah Street from NE Wheeler Avenue to NE 16th Street. In most places, the number of standard traffic lanes was reduced to add a bicycle lane with a painted "buffer" area, plastic "flexposts," and planters that separate the bikeway from the motor vehicle traffic lanes (see pictures below). The following questions are related to this protected bikeway.

NE Multnomah before and now:

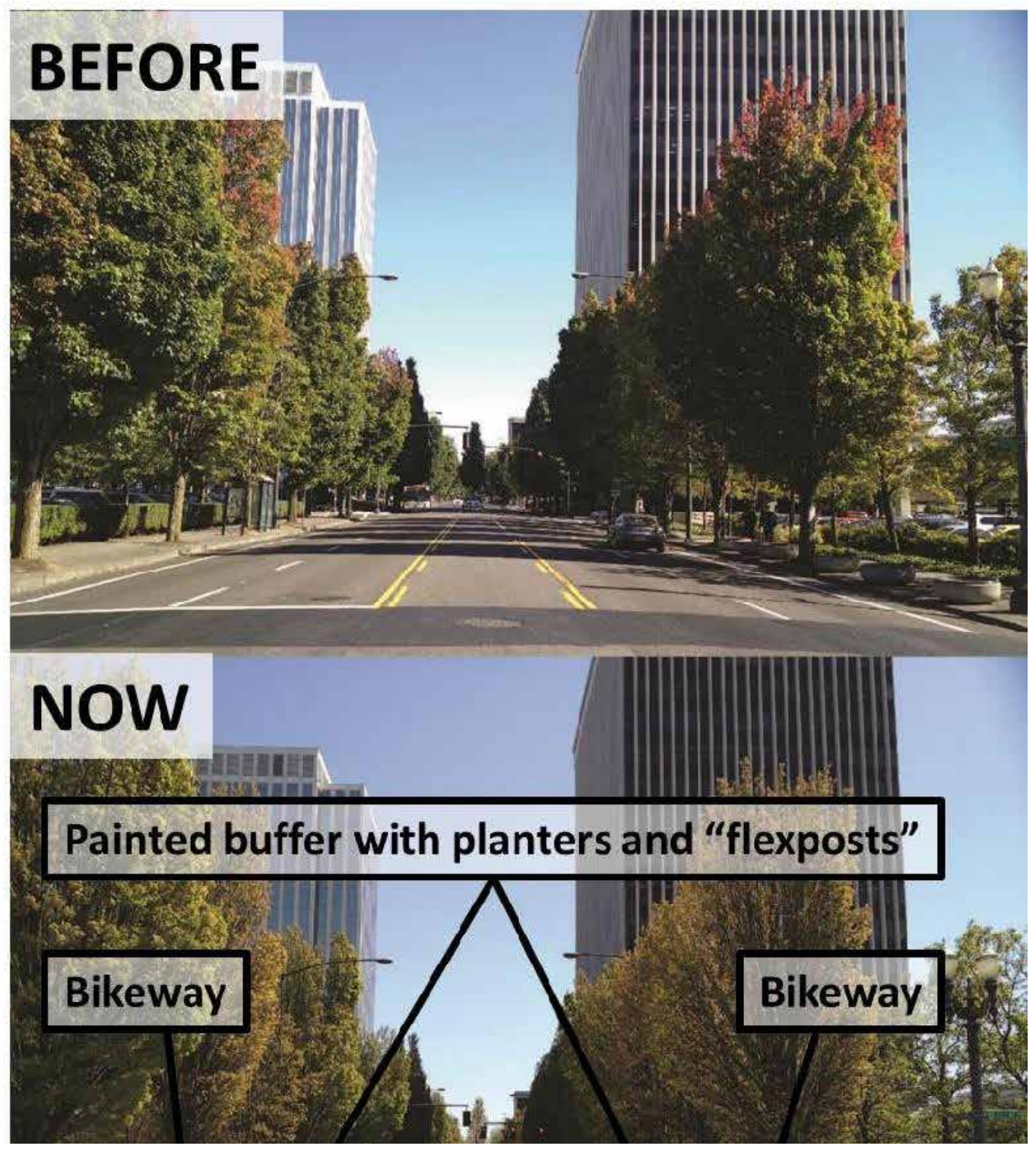




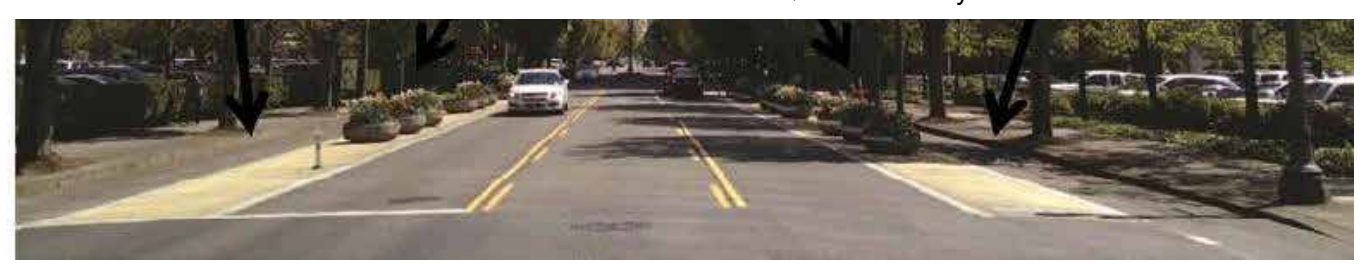

Extent of NE Multnomah protected bikeway

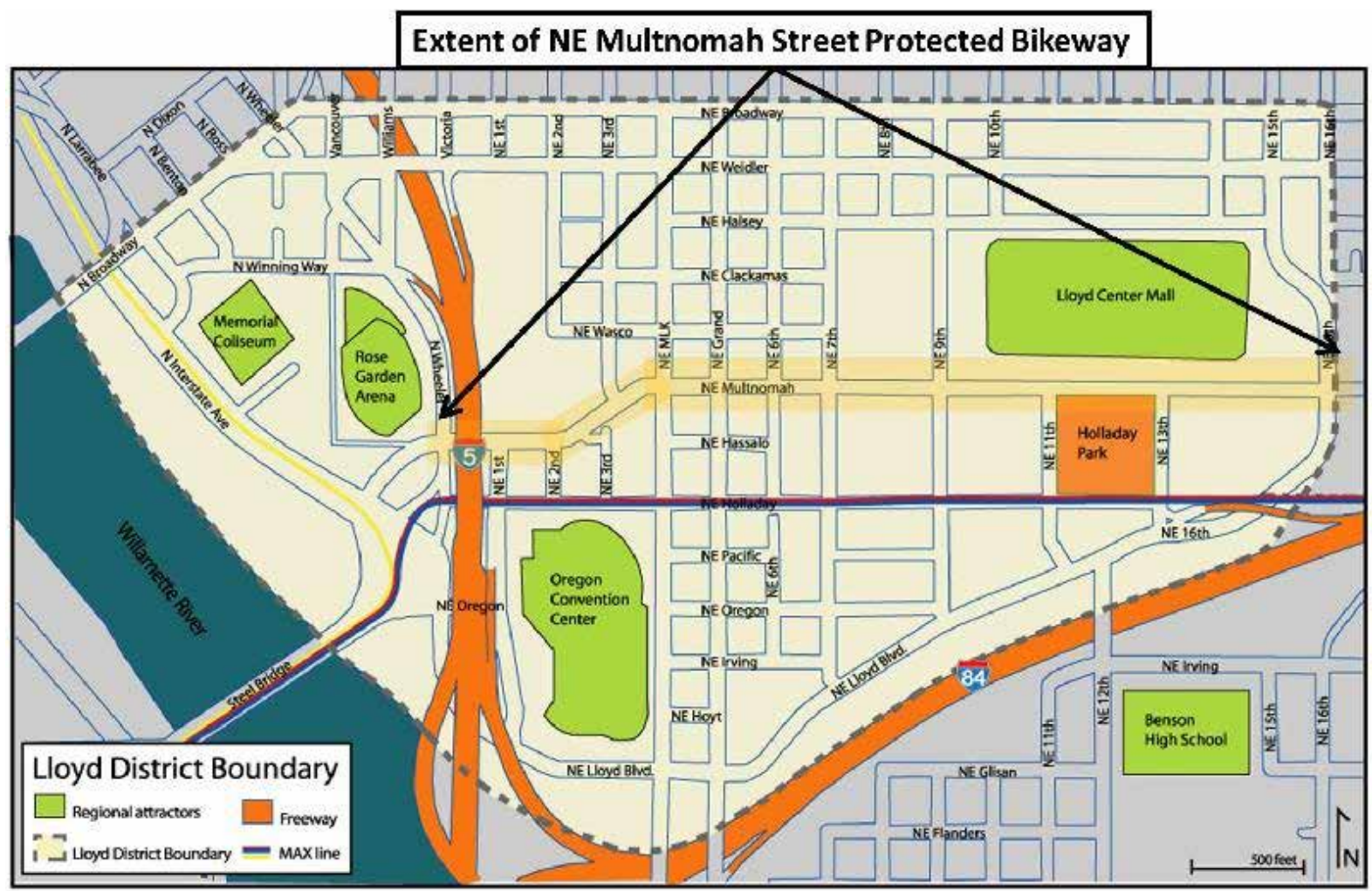

How often do you ride a bicycle on this part of NE Multnomah Street?
This was my first time
Less than once a month
A few times a month
A few times a week
Daily

How comfortable do you feel when bicycling in the NE Multnomah protected bikeway?
(1) Very Uncomfortable
(2)
(3)
(4)
(5)
(6) Very Comfortable 

$\checkmark$ LCDo "Счuсıиy
More frequently
About the same
This was my first time on this route

When bicycling on NE Multnomah Street between NE Wheeler Avenue and NE 16th Avenue, how often do you ride on the following:

\begin{tabular}{l|cccc} 
& Always & Most of the time & Some of the time & Never \\
\hline \begin{tabular}{l|l} 
Protected bikeway, including the \\
mixing area at intersections
\end{tabular} & 0 & 0 & 0 & 0 \\
Standard traffic lanes & 0 & 0 & 0 & 0 \\
Curb Area & 0 & 0 & 0
\end{tabular}

Consider the trip you were making when you were handed the postcard. Before the NE Multnomah protected bikeway was built, how would you have made this trip?
By bicycle, using this same route
By bicycle, using another route - (Please specify which route)
By foot
By public transportation
By car
By taxi
I would NOT have taken this trip
Other (please specify below)

\section{About Changes due to the Protected Bikeway}

Because of the NE Multnomah protected bikeway ...

Decreased Decreased Not Increased Increased No a Lot Somewhat Changed Somewhat a Lot Opinion

I feel the safety of bicycling on NE Multnomah Street has ... the time it takes me to bicycle on NE Multnomah has ... the usefulness of NE Multnomah for getting to places I want to go has how often I stop at shops and businesses on NE Multnomah has ...

how comfortable I feel when bicycling on NE Multnomah Street has . . drivers' awareness of people biking on NE Multnomah has ... drivers' speeds on NE Multnomah have... how often I ride a bicycle overall has ...

the difficulty of navigating around turning motor vehicles has... the difficulty of navigating around pedestrians has ... the difficulty of making a right off of NE Multnomah Street while bicycling has... 


\section{Your Opinions about the Protected Bikeway}

Please indicate if you agree or disagree with the following statements about the protected bikeway:

\begin{tabular}{|c|c|c|c|c|c|}
\hline & $\begin{array}{l}\text { Strongly } \\
\text { Disagree }\end{array}$ & $\begin{array}{l}\text { Somewhat } \\
\text { Disagree }\end{array}$ & $\begin{array}{l}\text { Somewhat } \\
\text { Agree }\end{array}$ & $\begin{array}{l}\text { Strongly } \\
\text { Agree }\end{array}$ & No Opinion \\
\hline $\begin{array}{l}\text { I would go out of my way to ride on NE Multnomah Street } \\
\text { compared to other streets. }\end{array}$ & 0 & 0 & 0 & 0 & 0 \\
\hline $\begin{array}{r}\text { Taking NE Multnomah is the most direct bicycle route to } \\
\text { my destination. }\end{array}$ & 0 & 0 & 0 & 0 & 0 \\
\hline $\begin{array}{r}\text { When bicycling on NE Multnomah, I always ride in the } \\
\text { protected bikeway. }\end{array}$ & 0 & 0 & 0 & 0 & 0 \\
\hline \multirow[t]{2}{*}{$\begin{array}{r}\text { The buffer section with planters between the traffic lanes } \\
\text { and the bikeway makes me feel safe. }\end{array}$} & 0 & 0 & 0 & 0 & 0 \\
\hline & $\begin{array}{l}\text { Strongly } \\
\text { Disagree }\end{array}$ & $\begin{array}{l}\text { Somewhat } \\
\text { Disagree }\end{array}$ & $\begin{array}{l}\text { Somewhat } \\
\text { Agree }\end{array}$ & $\begin{array}{l}\text { Strongly } \\
\text { Agree }\end{array}$ & No Opinion \\
\hline $\begin{array}{l}\text { The buffer section with plastic flexposts between the } \\
\text { traffic lanes and the bikeway makes me feel safe. }\end{array}$ & 0 & 0 & 0 & 0 & 0 \\
\hline $\begin{array}{l}\text { The buffer section with a painted/striped buffer between } \\
\text { the traffic lanes and the bikeway makes me feel safe. }\end{array}$ & 0 & 0 & 0 & 0 & 0 \\
\hline $\begin{array}{l}\text { Leaves and debris in the protected bikeway are worse } \\
\text { than other places I ride. }\end{array}$ & 0 & 0 & 0 & 0 & 0 \\
\hline \multirow[t]{2}{*}{$\begin{array}{r}\text { The protected bikeway is wide enough for me to ride } \\
\text { comfortably. }\end{array}$} & 0 & 0 & 0 & 0 & 0 \\
\hline & $\begin{array}{l}\text { Strongly } \\
\text { Disagree }\end{array}$ & $\begin{array}{l}\text { Somewhat } \\
\text { Disagree }\end{array}$ & $\begin{array}{l}\text { Somewhat } \\
\text { Agree }\end{array}$ & $\begin{array}{l}\text { Strongly } \\
\text { Agree }\end{array}$ & No Opinion \\
\hline $\begin{array}{r}\text { The protected bikeway is wide enough for one bicyclist to } \\
\text { pass another. }\end{array}$ & 0 & 0 & 0 & 0 & 0 \\
\hline $\begin{array}{l}\text { The protected bikeway is wide enough for two people to } \\
\text { comfortably ride side-by-side. }\end{array}$ & 0 & 0 & 0 & 0 & 0 \\
\hline $\begin{array}{r}\text { If I am bicycling with another adult, I would prefer to ride } \\
\text { side-by-side. }\end{array}$ & 0 & 0 & 0 & 0 & 0 \\
\hline $\begin{array}{r}\text { The protected bikeway is safer than other bikeways in } \\
\text { Portland. }\end{array}$ & 0 & 0 & 0 & 0 & 0 \\
\hline
\end{tabular}

Please indicate if you disagree or agree with the following statements about the effectiveness of the NE Multnomah protected bikeway:

\begin{tabular}{|c|c|c|c|c|c|}
\hline & $\begin{array}{l}\text { Strongly } \\
\text { Disagree }\end{array}$ & $\begin{array}{l}\text { Somewhat } \\
\text { Disagree }\end{array}$ & $\begin{array}{l}\text { Somewhat } \\
\text { Agree }\end{array}$ & $\begin{array}{l}\text { Strongly } \\
\text { Agree }\end{array}$ & No Opinion \\
\hline $\begin{array}{r}\text { The protected bikeway makes it clear where cars can be } \\
\text { and where the designated bicycle lanes are. }\end{array}$ & 0 & 0 & 0 & 0 & 0 \\
\hline $\begin{array}{l}\text { The intersection signals, signs, and street markings } \\
\text { make it clear who has the right-of-way at intersections. }\end{array}$ & 0 & 0 & 0 & 0 & 0 \\
\hline $\begin{array}{r}\text { The buffer, flexposts, and planters effectively separate } \\
\text { bikes from cars. }\end{array}$ & 0 & 0 & 0 & 0 & 0 \\
\hline $\begin{array}{r}\text { The buffer, flexposts, and planters do a good job at } \\
\text { protecting bikes from cars. }\end{array}$ & 0 & 0 & 0 & 0 & 0 \\
\hline $\begin{array}{r}\text { The protected bikeway makes drivers and bicyclists more } \\
\text { predictable. }\end{array}$ & 0 & 0 & 0 & 0 & 0 \\
\hline $\begin{array}{r}\text { The protected bikeway makes it clear where pedestrians } \\
\text { and bicyclists should be. }\end{array}$ & 0 & 0 & 0 & 0 & 0 \\
\hline $\begin{array}{r}\text { The protected bikeway design effectively separates } \\
\text { bicyclists from pedestrians. }\end{array}$ & 0 & 0 & 0 & 0 & 0 \\
\hline
\end{tabular}


What do you think it means when the pavement is painted yellow ("beeswax") along the bikeway? (Please enter your response in the box below.)

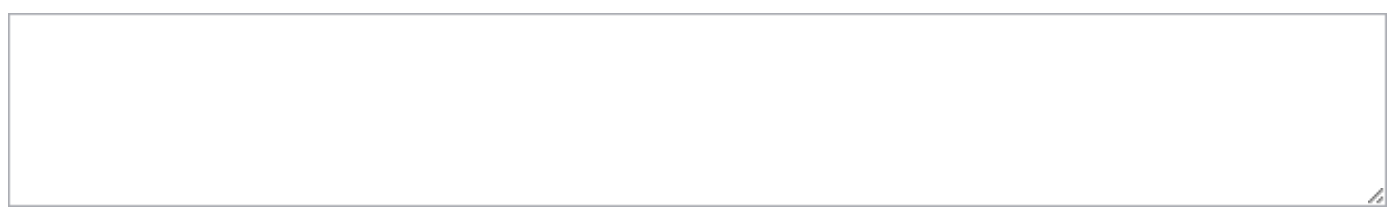

\section{About What you Encounter in the Protected Bikeway}

Please indicate how often you have observed the following on your rides in the NE Multnomah protected bikeway, AND whether you think this is a problem that needs to be addressed.

\begin{tabular}{|c|c|c|c|c|c|c|c|}
\hline & \multicolumn{4}{|c|}{$\begin{array}{l}\text { How often do you encounter the following } \\
\text { IN the protected bikeway? }\end{array}$} & \multicolumn{3}{|c|}{ How much of a problem is this? } \\
\hline & Never & Rarely & Sometimes & Often & $\begin{array}{l}\text { Not a } \\
\text { problem }\end{array}$ & $\begin{array}{l}\text { Minor } \\
\text { problem }\end{array}$ & $\begin{array}{l}\text { Major } \\
\text { Problem }\end{array}$ \\
\hline Cars parking & 0 & 0 & 0 & 0 & O & 0 & 0 \\
\hline $\begin{array}{r}\text { Cars loading or } \\
\text { unloading passengers }\end{array}$ & 0 & 0 & 0 & 0 & ○ & 0 & 0 \\
\hline $\begin{array}{l}\text { Delivery vehicles } \\
\text { loading or unloading }\end{array}$ & 0 & 0 & 0 & 0 & 0 & ○ & 0 \\
\hline Taxis & 0 & 0 & 0 & O & 0 & 0 & 0 \\
\hline $\begin{array}{l}\text { Cars/trucks driving } \\
\text { where they are not } \\
\text { supposed to (in the } \\
\text { protected bikeway) }\end{array}$ & O & 0 & O & 0 & $\bigcirc$ & 0 & ○ \\
\hline \multirow[t]{2}{*}{$\begin{array}{l}\text { Cars/trucks waiting to } \\
\text { make turns OFF of } \\
\text { NE Multnomah Street }\end{array}$} & 0 & 0 & O & 0 & O & 0 & 0 \\
\hline & Never & Rarely & Sometimes & Often & $\begin{array}{l}\text { Not a } \\
\text { problem }\end{array}$ & $\begin{array}{l}\text { Minor } \\
\text { problem }\end{array}$ & $\begin{array}{l}\text { Major } \\
\text { Problem }\end{array}$ \\
\hline $\begin{array}{l}\text { Cars/trucks waiting to } \\
\text { pull out ONTO NE } \\
\text { Multnomah Street }\end{array}$ & O & O & 0 & 0 & 0 & 0 & ○ \\
\hline $\begin{array}{l}\text { People walking in the } \\
\text { protected bikeway }\end{array}$ & 0 & 0 & 0 & 0 & 0 & 0 & 0 \\
\hline $\begin{array}{r}\text { People standing in } \\
\text { the protected } \\
\text { bikeway while waiting } \\
\text { to cross the street }\end{array}$ & $\bigcirc$ & $\bigcirc$ & 0 & 0 & 0 & 0 & 0 \\
\hline $\begin{array}{l}\text { Bicyclists traveling in } \\
\text { the WRONG direction }\end{array}$ & 0 & 0 & 0 & 0 & 0 & 0 & 0 \\
\hline $\begin{array}{l}\text { Buses loading and } \\
\text { unloading passengers }\end{array}$ & 0 & 0 & 0 & 0 & 0 & 0 & 0 \\
\hline
\end{tabular}

\section{About What You Encounter in the Protected Bikeway}

At certain points along the NE Multnomah protected bikeway, the buffer is discontinued for a short distance and buses pull over to the curb to load and unload (see photo below).

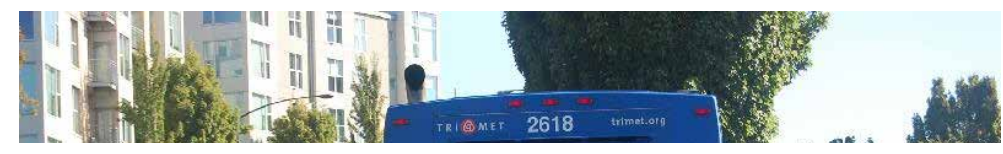




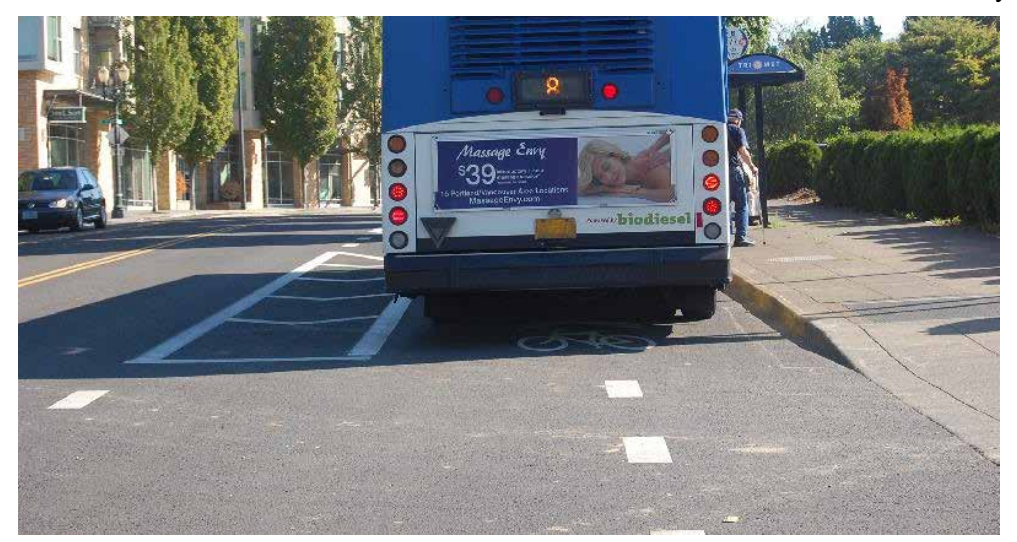

If you encountered this bus stopped in front of you, what would you do:

Stop and wait for the bus to move

Go around the bus on the left

Go up onto the sidewalk to go around

Other (please describe)

While riding on the NE Multnomah protected bikeway, have you ever had collisions or near-collisions with other people or objects on the road?

Yes, a collision

Yes, a near-collision

No, neither

If you have been involved in a collision or near-collision, please indicate what other people or objects were involved?

(Check all that apply)

\begin{tabular}{|c|c|c|}
\hline & Collision & Near Collision \\
\hline Another bicyclist & $\square$ & $\square$ \\
\hline A pedestrian & 回 & $\square$ \\
\hline A turning car & $\square$ & 回 \\
\hline A parking car & $\square$ & $\square$ \\
\hline A parked car & $\square$ & $\square$ \\
\hline A delivery truck & $\square$ & $\square$ \\
\hline A bus & $\square$ & $\square$ \\
\hline A taxi & $\square$ & $\square$ \\
\hline One of the concrete planters & $\square$ & $\square$ \\
\hline $\begin{array}{r}\text { One of the protected bikeway } \\
\text { plastic flexposts }\end{array}$ & $\square$ & $\square$ \\
\hline $\begin{array}{r}\text { Other stationary object (please } \\
\text { describe). }\end{array}$ & $\square$ & $\square$ \\
\hline Something else (please describe). & & \\
\hline
\end{tabular}

Please provide a brief description of the collision(s) you were involved in while riding a bicycle in the protected bikeway. 


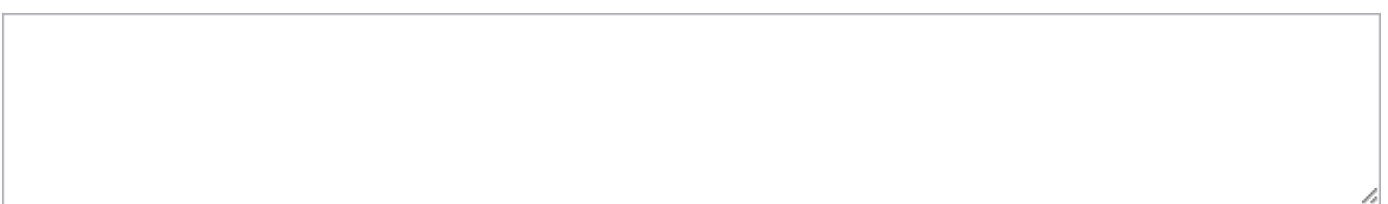

\section{About Intersections}

At some intersections along NE Multnomah Street, the buffer ends as the bikeway approaches the intersection (see photo below).

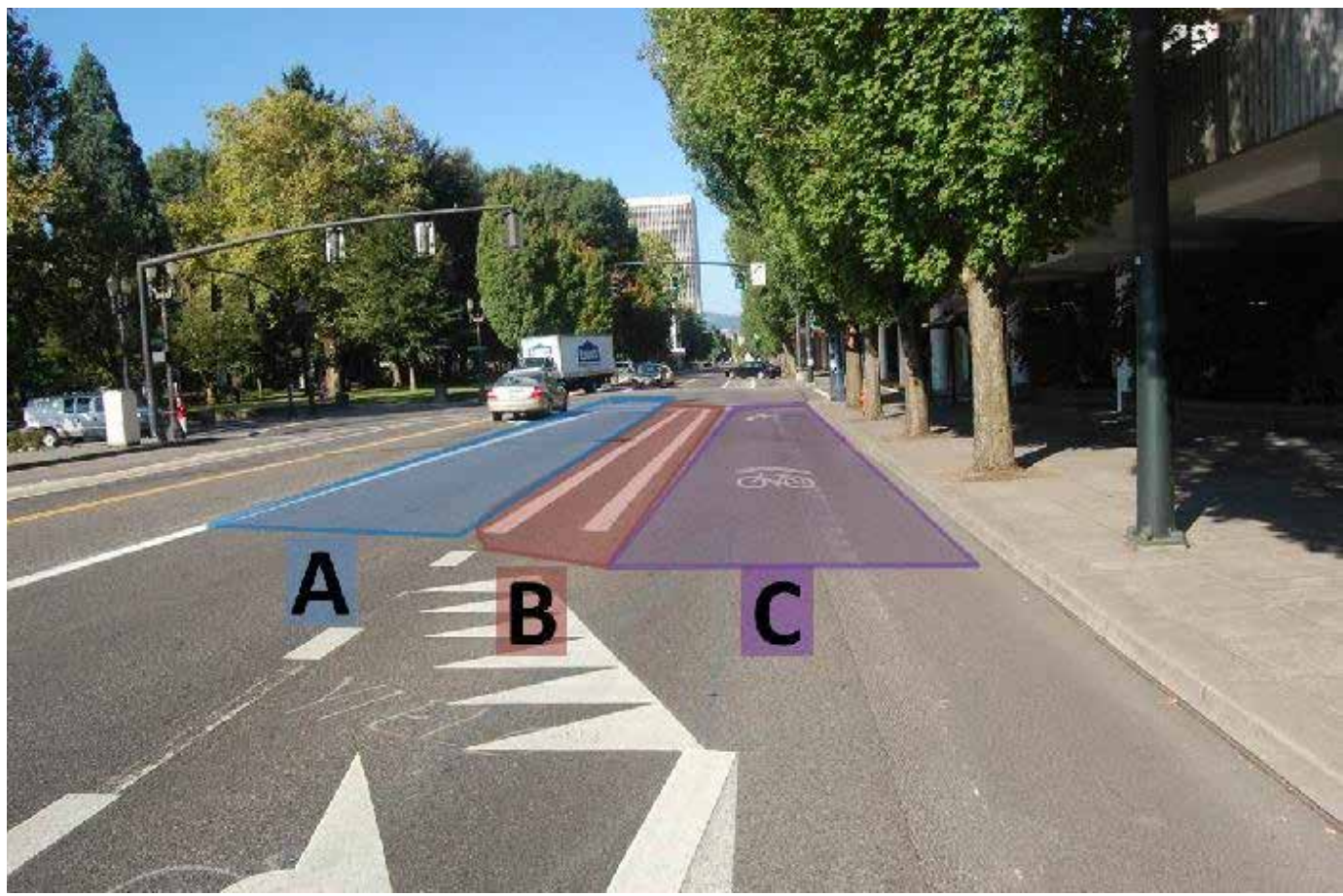

Please indicate whether you think the person should be in the space designated by A, B, or C for each scenario (select all that apply)...

\begin{tabular}{r|r|} 
A motorist turning right \\
should be in ...
\end{tabular}

Referring to the photo above, please indicate your level of agreement with the following statements:

\begin{tabular}{|c|c|c|c|c|c|}
\hline & $\begin{array}{l}\text { Strongly } \\
\text { Disagree }\end{array}$ & $\begin{array}{l}\text { Somewhat } \\
\text { Disagree }\end{array}$ & $\begin{array}{l}\text { Somewhat } \\
\text { Agree }\end{array}$ & Strongly Agree & No Opinion \\
\hline $\begin{array}{r}\text { I understand where I am supposed to ride when } \\
\text { approaching the intersection. }\end{array}$ & 0 & 0 & 0 & 0 & 0 \\
\hline $\begin{array}{r}\text { Right-turning motorists generally yield to bicyclists who } \\
\text { are continuing straight. }\end{array}$ & 0 & 0 & 0 & 0 & ○ \\
\hline $\begin{array}{r}\text { Motorists generally understand how to make right turns } \\
\text { at these intersections. }\end{array}$ & 0 & 0 & 0 & 0 & 0 \\
\hline Right-turning cars rarely block my pathway when I am & $\curvearrowright$ & $\curvearrowright$ & $\curvearrowleft$ & $\curvearrowright$ & $\curvearrowright$ \\
\hline
\end{tabular}




\section{About Intersections (continued)}

At some intersections along NE Multnomah Street, the painted buffer continues to the intersection and right-turning motor vehicles make their right turns from the standard traffic lane to the left of the buffer. There are green painted markings in the intersection. (See picture below).

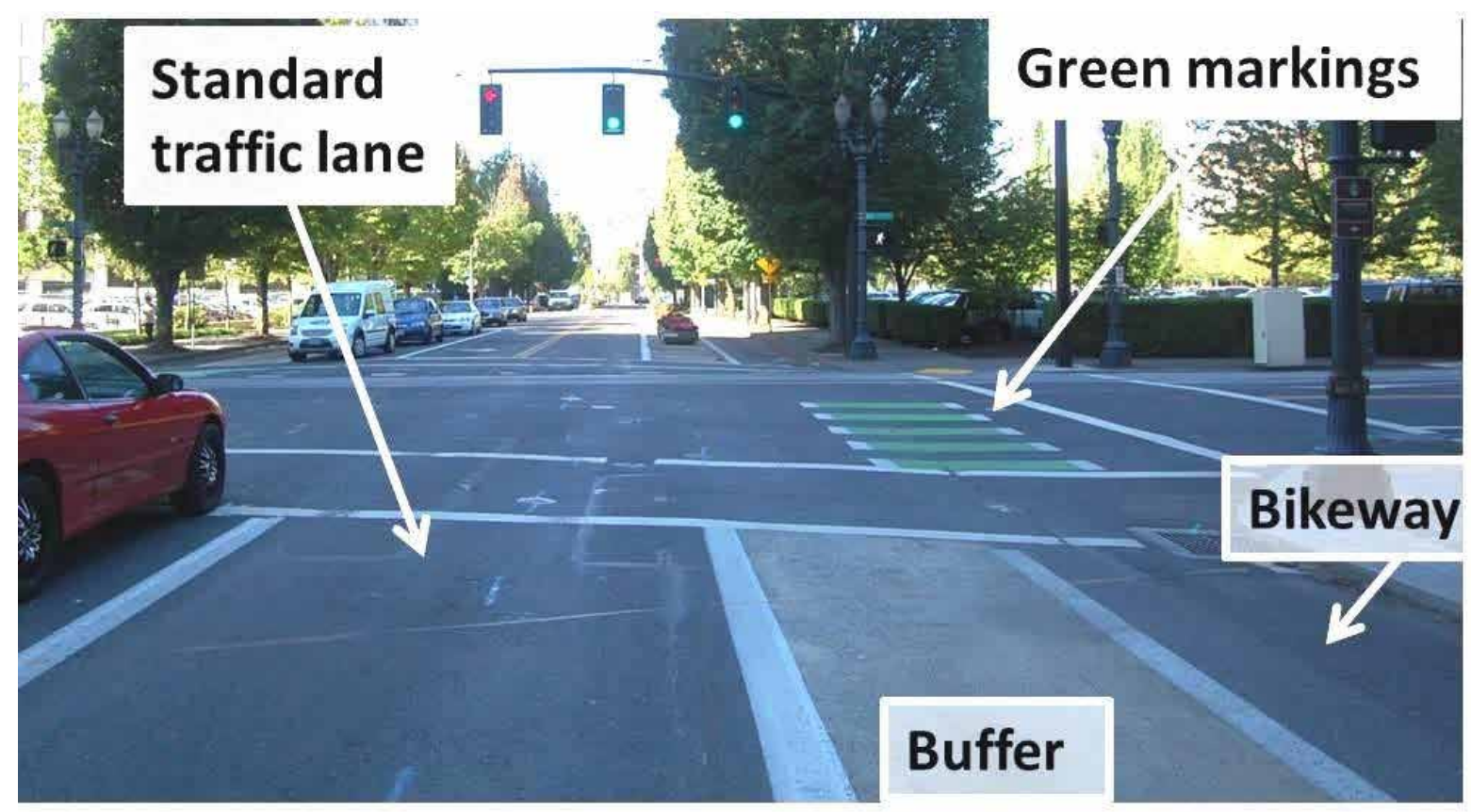

Indicate your level of agreement with the following statements

\begin{tabular}{|c|c|c|c|c|c|}
\hline & $\begin{array}{l}\text { Strongly } \\
\text { Disagree }\end{array}$ & $\begin{array}{l}\text { Somewhat } \\
\text { Disagree }\end{array}$ & $\begin{array}{l}\text { Somewhat } \\
\text { Agree }\end{array}$ & Strongly Agree & No Opinion \\
\hline $\begin{array}{l}\text { Turning motorists generally yield to bicyclists when } \\
\text { bicyclists are going straight and cars are turning right. }\end{array}$ & 0 & 0 & 0 & ○ & 0 \\
\hline $\begin{array}{r}\text { Motorists generally understand how to make right turns } \\
\text { at these intersections. }\end{array}$ & 0 & 0 & 0 & 0 & 0 \\
\hline $\begin{array}{r}\text { Cars rarely block my pathway through these } \\
\text { intersections. }\end{array}$ & 0 & 0 & 0 & 0 & 0 \\
\hline $\begin{array}{l}\text { I generally feel safe when bicycling straight at these } \\
\text { intersections when cars are turning right. }\end{array}$ & 0 & 0 & 0 & 0 & 0 \\
\hline
\end{tabular}


Do you ever use the NE Multnomah protected bikeway to head south and west (e.g. to get downtown, to the Steel Bridge, and/or the Eastbank Esplanade)?
Yes
No
I'm not sure

When you head south and west, which path do you usually take to get toward downtown, the Steel Bridge, and/or the Eastbank Esplanade?

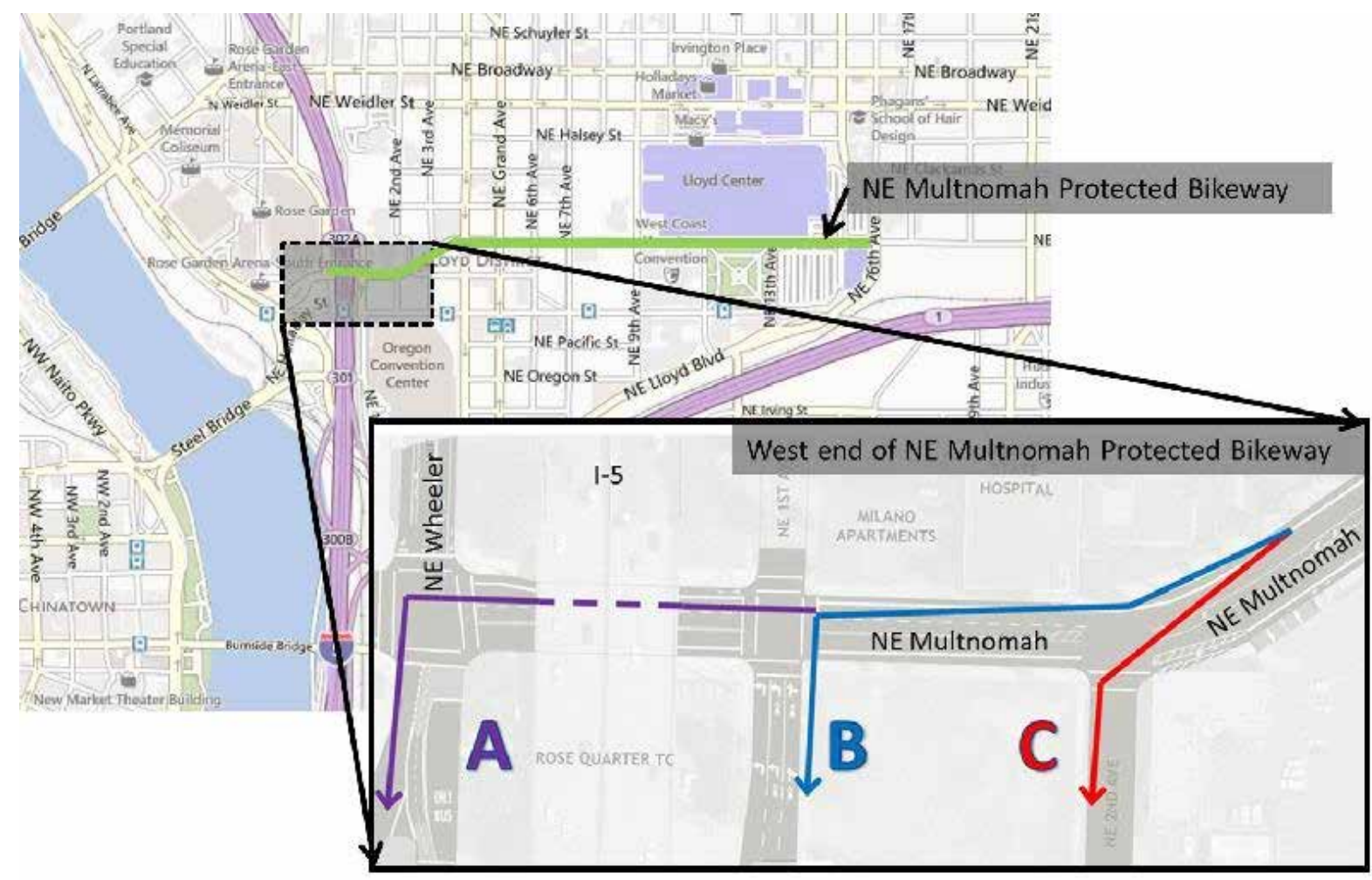
I continue on NE Multnomah and go left at NE Wheeler (Path A)
I go left at 1st Avenue (Path B)
I merge out of the protected bikeway and go left at 2nd Avenue (Path C)
Other (please describe)

\section{Loading and Drop-Off Zone}

At one mid-block location between 6th and 7th Avenues, there is a pull-out for vehicles to load and unload and to access the metered parking spots. (See photo below)

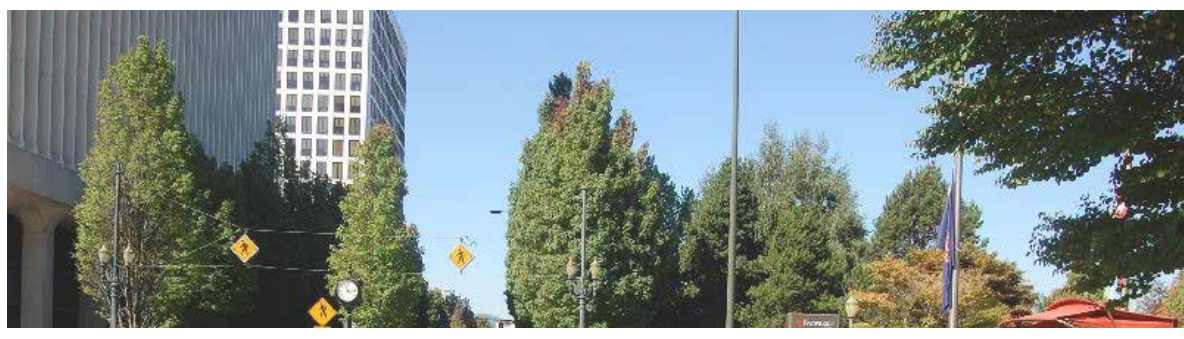




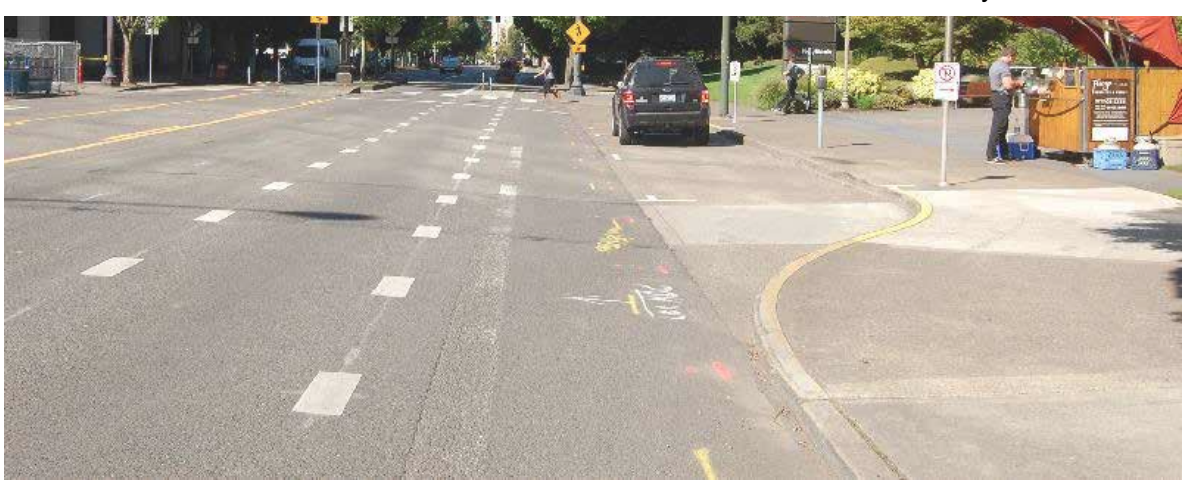

Please indicate if you agree or disagree with the following statements about the loading zone:

\begin{tabular}{|c|c|c|c|c|c|}
\hline & $\begin{array}{l}\text { Strongly } \\
\text { Disagree }\end{array}$ & $\begin{array}{l}\text { Somewhat } \\
\text { Disagree }\end{array}$ & $\begin{array}{l}\text { Somewhat } \\
\text { Agree }\end{array}$ & $\begin{array}{l}\text { Strongly } \\
\text { Agree }\end{array}$ & No Opinion \\
\hline $\begin{array}{r}\text { I often encounter motor vehicles pulling into or out of } \\
\text { this location. }\end{array}$ & 0 & 0 & 0 & 0 & $\bigcirc$ \\
\hline $\begin{array}{l}\text { Stopped vehicles at this loading zone usually pull all the } \\
\text { way into the pull-out and allow enough space for } \\
\text { bicyclists to pass on the left. }\end{array}$ & ○ & 0 & 0 & 0 & 0 \\
\hline $\begin{array}{l}\text { Most motorists pay attention to bicyclists when entering } \\
\text { and exiting the loading zone area. }\end{array}$ & 0 & 0 & 0 & 0 & ○ \\
\hline $\begin{array}{l}\text { I feel comfortable bicycling around vehicles in the } \\
\text { loading zone. }\end{array}$ & 0 & 0 & 0 & 0 & 0 \\
\hline
\end{tabular}

\section{Overall Impressions of the Protected Bikeway}

What is the best thing about the protected bikeway?

What should be improved about the protected bikeway?

\section{About Bicycling and Comfort}

Regardless of whether you currently bicycle in all of the following situations, please consider how comfortable you would be riding a bicycle in each place:

$\mid \begin{array}{cccccc}\begin{array}{c}\text { Very } \\ \text { Uncomfortable } \\ (1)\end{array} & \text { (2) } & \text { (3) } & \text { (4) } & \text { (5) } & \begin{array}{c}\text { Very } \\ \text { Comfortable } \\ (6)\end{array}\end{array}$

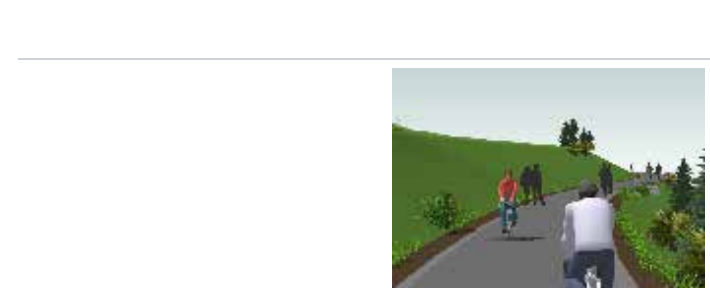

(1) 
(A) On a path or trail separate from the street

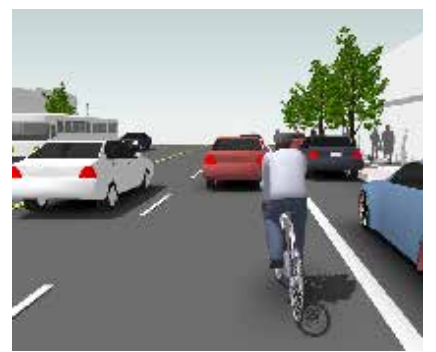

(B) On a commerciaNE Multnomah Street with two lanes of traffic in each direction, with traffic speeds of 35 miles per hour, on-street car parking, and no bikeway

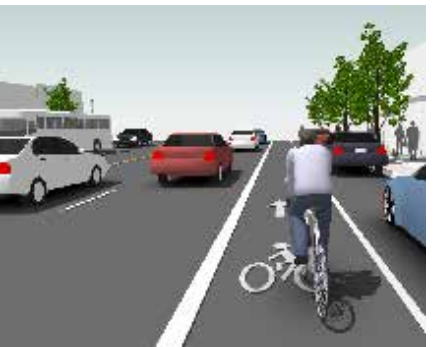

(C) On a similar street to (B), but with a striped bikeway added

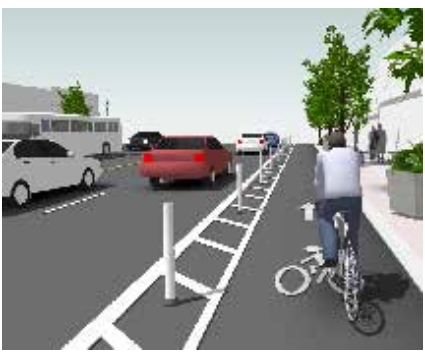

(D) On a similar street to (B), but with a physically separated bikeway
Very

Uncomfortable

(1)

(2)

(3)

(4)

(5)

Comfortable

(6)

How comfortable would you feel bicycling on a commercial street with two lanes of traffic in each direction, with traffic speeds of 35 miles per hour (Situation D above), but with the following types of separation from traffic:

Very Uncomfortable

(1)

(1)

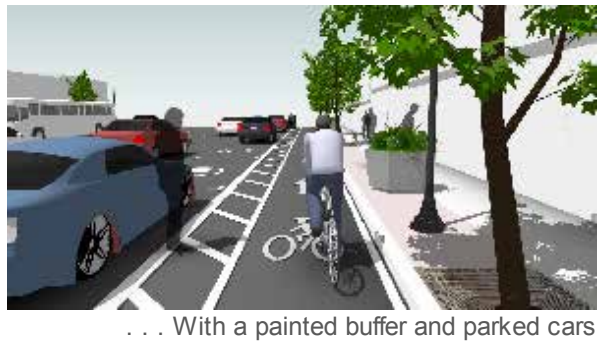

$(2)$

(3)

(4)

(5)

Very Comfortable
(6) 


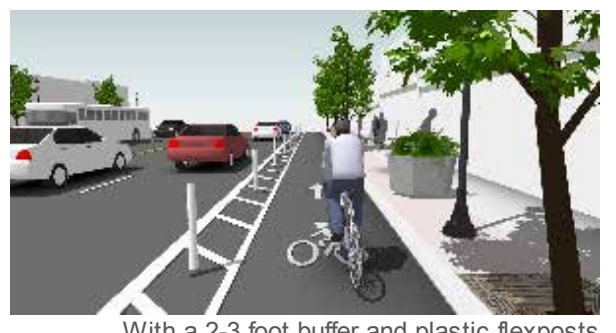

With a 2-3 foot buffer and plastic flexposts

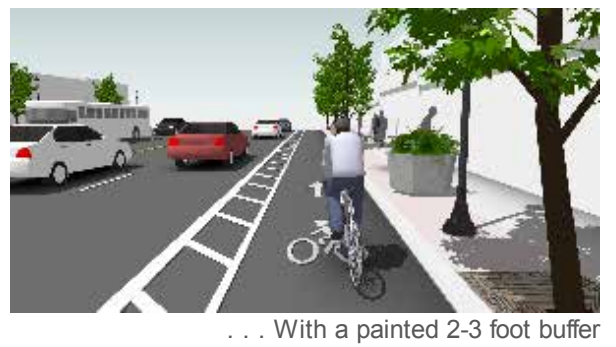

Very

Uncomfortable

(1)
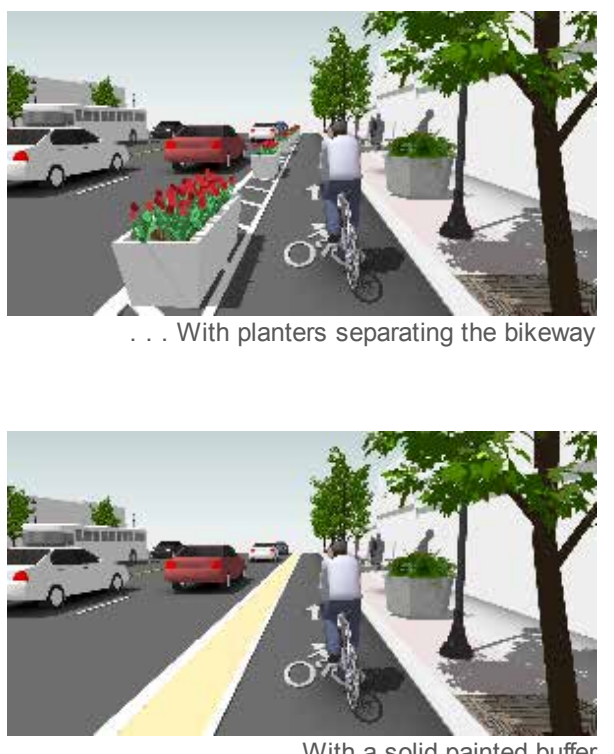

. With a solid painted buffer

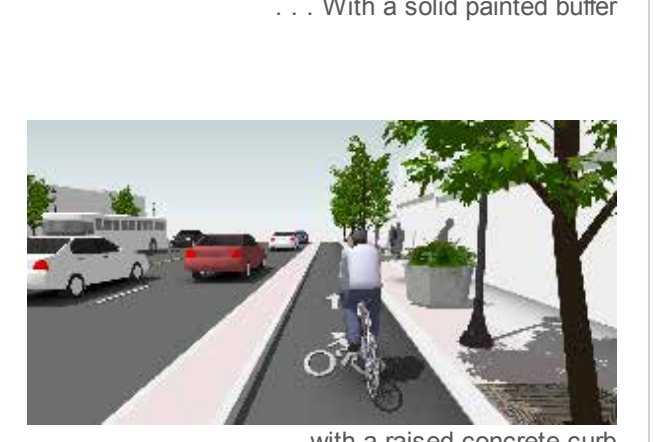

... with a raised concrete curb

O

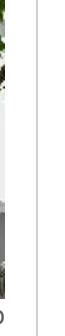

O

O

$(2$

(2)

(3)

(4)

(5)

Very Comfortable

(6)

0 
Indicate if you agree or disagree with the following statements:

\begin{tabular}{|c|c|c|c|c|c|}
\hline & $\begin{array}{l}\text { Strongly } \\
\text { Disagree }\end{array}$ & $\begin{array}{l}\text { Somewhat } \\
\text { Disagree }\end{array}$ & Somewhat Agree & Strongly Agree & No Opinion \\
\hline Most drivers follow the rules of the road. & O & 0 & 0 & ○ & 0 \\
\hline Most drivers are predictable. & 0 & 0 & O & 0 & 0 \\
\hline Most bicyclists follow the rules of the road. & 0 & 0 & O & O & 0 \\
\hline Most bicyclists are predictable. & ○ & 0 & 0 & ○ & 0 \\
\hline Most pedestrians follow the rules of the road. & 0 & 0 & 0 & 0 & 0 \\
\hline Most pedestrians are predictable. & 0 & 0 & 0 & 0 & 0 \\
\hline
\end{tabular}

\section{About You}

We have a few questions about you so that we may understand the characteristics of our survey respondents.

Do you have a current:

\begin{tabular}{r|cc} 
& Yes & No \\
\hline Driver's License & 0 & 0 \\
Transit Pass & 0 & 0
\end{tabular}

How many working motor vehicles does your household own or lease? (Do not include motorhomes).

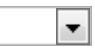

How many working adult bicycles does your household own?

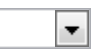

What is your home zip code?

5 digit zip code:

INCLUDING YOURSELF, how many people live in your household?

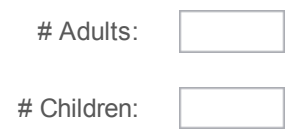

\# Children:

What is your gender?

Male

○
Female

○ 
What is your age?

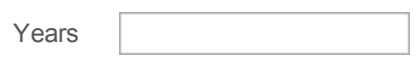

Do you consider yourself: (select all that apply)

$\square$ American Indian or Alaska Native

$\square$ Asian

$\square$ Black or African American

$\square$ Hispanic or Latino/a

White or Caucasian

$\square$ Other:

What is your employment status? (Select all that apply)

I work outside the home -> What is the zip code of your place of work?

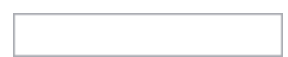

I go to school outside the home -> What is the zip code of your school?

I work from home

Not employed at this time

What is your annual household income?

Less than $\$ 25,000$

$\$ 25,000$ to less than $\$ 50,000$

$\$ 50,000$ to less than $\$ 75,000$

$\$ 75,000$ to less than $\$ 100,000$

$\$ 100,000$ to less than $\$ 200,000$

$\$ 200,000$ or more

I prefer not to provide this information

What is the highest level of school you havecompleted?

Some high school or less

O

High school diploma or GED

Some College

Trade/Vocational School

Associate Degree

Four-year college degree or more

Other (please specify): 
Is there anything else that you would like to tell us?

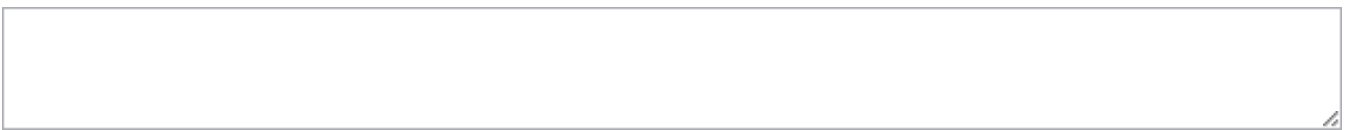

That's all the questions we have. Would you like to be entered into a drawing for one of three $\$ 100$ gift cards to Amazon.com?

(2) Yes, I would like to be entered in the drawing (I'll enter my name on the next page).

No thanks. I will complete my survey by clicking the ">>" button below

Enter your name and some way for us to get a hold of you below. We will only use this information to contact you if your entry is selected to receive a gift card. This information will not be associated with any of your survey responses.
Name
Phone or email

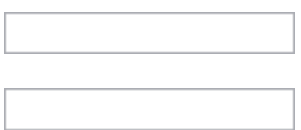

Thanks for taking the survey - please make sure to click the ">>" button below to submit your entry and close the survey! 


\section{BICYCLIST: SAN FRANCISCO - OAK AND FELL STREETS}




\section{Neighborhood Street Study}

Default Question Block

Dear Bicyclist,

My name is Chris Monsere and I am a faculty member at Portland State University in the Department of Civil and Environmental Engineering. I am the principal investigator on a project researching separated bikeways in six cities across the country.

Hearing from bicyclists like yourself is a very important part of this study. We are only sampling a select number of bicyclists near the Oak and Fell Street separated bikeways (also known as "cycle tracks"). Therefore, every response is very important and we hope you will participate. We will share our findings with the City of San Francisco and hope that the results will help in future plans for improving bicycling in cities around the United States.

The survey, which starts on the next page, should take about 20 minutes. Your participation in the study is voluntary and you may stop taking the survey at any time with no consequences.

The postcard that you received has a unique number that only identifies where and when we handed you the postcard. We will protect the confidentiality of your individual survey responses. None of your responses will be linked to your name or other identifying personal information.

To say thank you, all people who complete their survey by 09/09/2013 will be entered into a drawing for one of three $\$ 100$ Amazon.com gift cards. You will be asked to enter your name and a way to contact you at the end of the survey - this information is voluntary and will not be connected to your survey data.

If you have concerns or problems about your participation in this study or your rights as a research subject, please contact the Human Subjects Research Review Committee, Office of Research and Strategic Partnerships, Market Center Building Suite 620, Portland State University, (877-480-4400). If you have questions about the study itself, please contact our research team directly at streets@pdx.edu or 503-725-2875.

Sincerely,

Christopher M. Monsere, Ph.D., P.E.

Associate Professor \& Associate Chair

Civil and Environmental Engineering

Portland State University

Do you agree to participate in this survey?

No

Yes

When you received the postcard for this survey, where were you ...

\begin{tabular}{|c|ccccc|c|} 
& & & Place & & Can you provide an address or \\
closest intersection to this place?
\end{tabular}

If you selected other, please explain:

When you received the postcard for this survey, were you riding on ...
Oak Street
Fell Street
Other 
What type of bicycle were you riding when you received the postcard?
Personal Bicycle
Bike Share Bicycle
Rental Bicycle
Other (please specify):

\section{Oak Street Separated Bikeway}

A separated bikeway, also known as a "cycle track", was recently built on Oak Street between Baker Street and Scott Street. Bike lanes are separated from other traffic lanes by a combination of a striped "buffer" zone and plastic "flexposts" (see picture below).
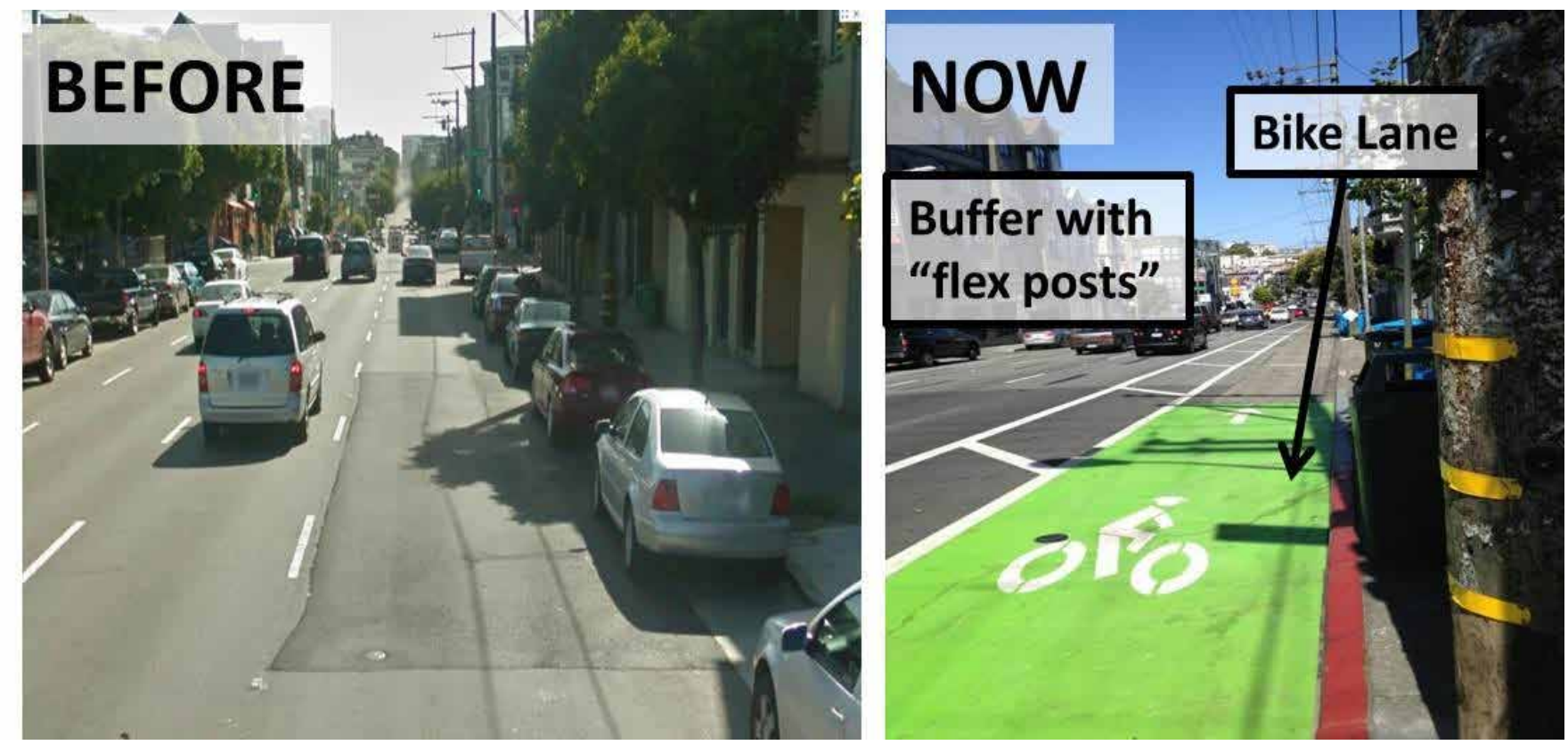

The next set of questions is about the Oak Street separated bikeway.

Have you ridden a bicycle in the Oak Street separated bikeway since it was installed?

Yes

No

How often do you ride a bicycle on this part of Oak Street?
This was my first time
Less than once a month
A few times a month
A few times a week
Daily

How comfortable do you feel when bicycling in the Oak Street separated bikeway?

(1) Very Uncomfortable

$\therefore$ in 

$\odot(<)$
(3)
(4)
(5)
(6) Very Comfortable

Since the Oak Street separated bikeway was built, do you travel on this route:
Less frequently
More frequently
About the same
This was my first time on this route

When bicycling on Oak Street between Baker Street and Scott Street, how often you ride on the following:

\begin{tabular}{l|cccc} 
& Always & Most of the time & Some of the time & Never \\
\hline Separated bikeway & 0 & 0 & 0 & 0 \\
Standard traffic lanes & 0 & 0 & 0 & 0 \\
In the gutter, near the curb & 0 & 0 & 0
\end{tabular}

Consider the trip you were making when you were handed the postcard. Before the Oak Street separated bikeway was built, how would you have made this trip?
By bicycle, using this same route
By bicycle, using another route - (Please specify which route):
By foot
By public transportation
By car
By taxi
I would NOT have taken this trip
Other (please specify below)

\section{About Changes Due to the Separated Bikeway}

Because of the Oak Street separated bikeway ...

\begin{tabular}{|c|c|c|c|c|c|c|}
\hline & $\begin{array}{c}\text { Decreased a } \\
\text { Lot }\end{array}$ & $\begin{array}{l}\text { Decreased } \\
\text { Somewhat }\end{array}$ & $\begin{array}{c}\text { Not } \\
\text { Changed }\end{array}$ & $\begin{array}{l}\text { Increased } \\
\text { Somewhat }\end{array}$ & $\begin{array}{l}\text { Increased a } \\
\text { Lot }\end{array}$ & No Opinion \\
\hline I feel the safety of bicycling on Oak Street has ... & O & O & O & 0 & 0 & 0 \\
\hline the time it takes me to bicycle on Oak Street has... & O & 0 & 0 & 0 & 0 & 0 \\
\hline the usefulness of Oak Street for getting to places I want to go has ... & O & 0 & 0 & O & 0 & 0 \\
\hline how often I stop at shops and businesses on Oak Street has ... & 0 & 0 & 0 & 0 & 0 & 0 \\
\hline how comfortable I feel when bicycling on Oak Street has ... & $\bigcirc$ & 0 & 0 & 0 & 0 & ○ \\
\hline drivers' awareness of people biking on Oak Street has ... & 0 & 0 & 0 & 0 & 0 & 0 \\
\hline drivers' speeds on Oak Street have... & 0 & 0 & $\bigcirc$ & 0 & 0 & ○ \\
\hline how often I ride a bicycle overall has ... & 0 & 0 & 0 & 0 & 0 & 0 \\
\hline the difficulty of navigating around turning motor vehicles has ... & 0 & 0 & 0 & 0 & 0 & 0 \\
\hline the difficulty of navigating around pedestrians has ... & ○ & ○ & 0 & 0 & $\bigcirc$ & ○ \\
\hline
\end{tabular}


Please indicate if you agree or disagree with the following statements about the Oak Street separated bikeway:

\begin{tabular}{|c|c|c|c|c|c|}
\hline & $\begin{array}{l}\text { Strongly } \\
\text { Disagree }\end{array}$ & $\begin{array}{l}\text { Somewhat } \\
\text { Disagree }\end{array}$ & Somewhat Agree & Strongly Agree & No Opinion \\
\hline $\begin{array}{l}\text { I would go out of my way to ride on Oak Street compared } \\
\text { to other streets. }\end{array}$ & 0 & ○ & 0 & 0 & 0 \\
\hline $\begin{array}{r}\text { Taking Oak Street is the most direct bicycle route to my } \\
\text { destination. }\end{array}$ & 0 & 0 & 0 & 0 & 0 \\
\hline $\begin{array}{c}\text { The buffer with the striped paint and plastic flexposts } \\
\text { between the traffic lanes and the bikeway makes me feel } \\
\text { safe. }\end{array}$ & 0 & 0 & 0 & 0 & 0 \\
\hline $\begin{array}{l}\text { Leaves and debris in the separated bikeway are worse } \\
\text { than other places I ride. }\end{array}$ & 0 & 0 & ○ & 0 & ○ \\
\hline $\begin{array}{l}\text { The separated bikeway is wide enough for me to ride } \\
\text { comfortably. }\end{array}$ & 0 & 0 & 0 & 0 & ○ \\
\hline $\begin{array}{r}\text { The separated bikeway is wide enough for one bicyclist to } \\
\text { pass another. }\end{array}$ & 0 & 0 & 0 & 0 & 0 \\
\hline $\begin{array}{l}\text { The separated bikeway is wide enough for two people to } \\
\text { comfortably ride side-by-side. }\end{array}$ & 0 & ○ & ○ & 0 & 0 \\
\hline $\begin{array}{r}\text { If I am bicycling with another adult, I would prefer to ride } \\
\text { side-by-side. }\end{array}$ & 0 & ○ & 0 & 0 & ○ \\
\hline $\begin{array}{r}\text { The separated bikeway is safer than other bike lanes in } \\
\text { San Francisco. }\end{array}$ & 0 & 0 & 0 & 0 & 0 \\
\hline
\end{tabular}

Please indicate if you agree or disagree with the following statements about the effectiveness of the Oak Street separated bikeway:

\begin{tabular}{|c|c|c|c|c|c|}
\hline & $\begin{array}{l}\text { Strongly } \\
\text { Disagree }\end{array}$ & $\begin{array}{l}\text { Somewhat } \\
\text { Disagree }\end{array}$ & Somewhat Agree & Strongly Agree & No Opinion \\
\hline $\begin{array}{l}\text { The separated bikeway makes it clear where cars can be } \\
\text { and where the designated bicycle lanes are. }\end{array}$ & 0 & 0 & 0 & 0 & 0 \\
\hline $\begin{array}{l}\text { The intersection signals, signs, and street markings } \\
\text { make it clear who has the right-of-way at intersections. }\end{array}$ & 0 & 0 & 0 & 0 & O \\
\hline The buffer effectively separates bikes from cars. & 0 & 0 & 0 & 0 & 0 \\
\hline The buffer does a good job at protecting bikes from cars. & O & 0 & 0 & 0 & 0 \\
\hline $\begin{array}{r}\text { The separated bikeway makes drivers and bicyclists } \\
\text { more predictable. }\end{array}$ & 0 & 0 & 0 & O & 0 \\
\hline $\begin{array}{r}\text { The separated bikeway makes it clear where bicyclists } \\
\text { and pedestrians should be. }\end{array}$ & 0 & 0 & 0 & 0 & 0 \\
\hline $\begin{array}{r}\text { The separated bikeways' design effectively separates } \\
\text { bicyclists from pedestrians. }\end{array}$ & 0 & O & 0 & O & O \\
\hline
\end{tabular}

\section{Intersection of Oak Street and Divisadero Street}

At the intersection of Oak Street and Divisadero Street, the bikeway shifts out toward the motor vehicle traffic lane and right-turning motor vehicles cross the bike lane to complete their turns. This area is called a "mixing zone" (See picture below). Sometimes the green-marked bike lane is called an "advisory lane."

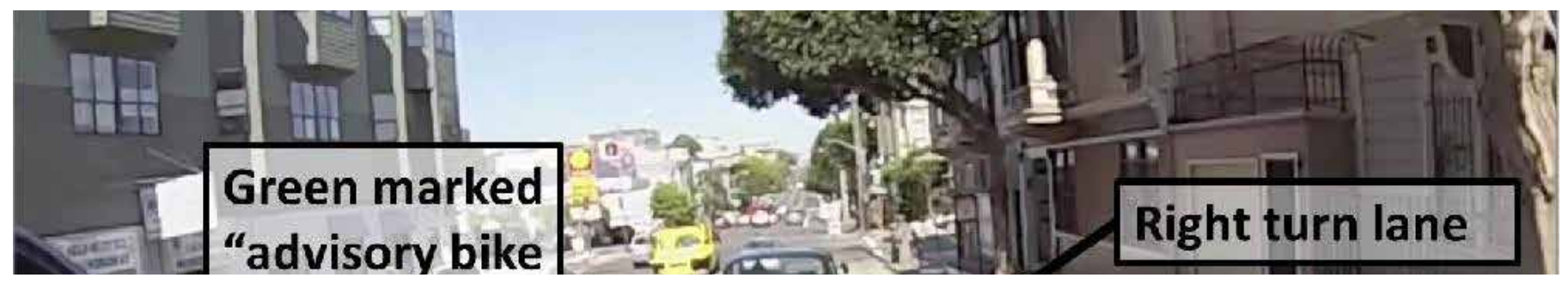




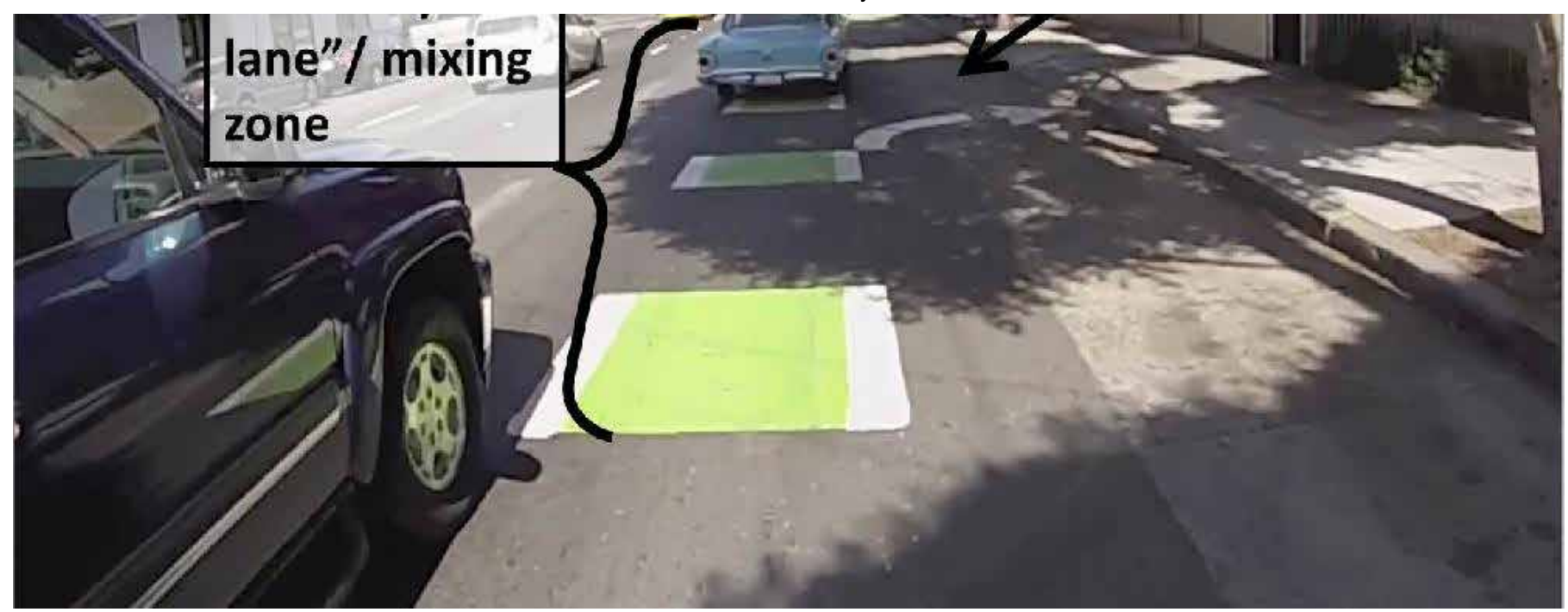

Indicate your level of agreement with the following statements

$\begin{array}{r}\text { I understand where I am supposed to ride when } \\ \text { approaching the intersection. }\end{array}$
$\begin{gathered}\text { I usually follow the bicycle lane marking and move over } \\ \text { to the left (into the green marked bike lane) when } \\ \text { approaching the intersection. }\end{gathered}$
$\begin{array}{r}\text { Turning motorists generally yield to bicyclists when } \\ \text { lane. }\end{array}$
$\begin{gathered}\text { moving through the mixing zone and into the right-turn } \\ \text { at these intersections. }\end{gathered}$
$\begin{gathered}\text { Cars rarely block my pathway through the mixing zone. } \\ \text { I often see motorists making right turns from the wrong } \\ \text { lane (i.e. the lanes to the left of the cycle track rather } \\ \text { than the right-turn lane). }\end{gathered}$

Intersection of Oak Street and Broderick Street

At the intersection of Oak Street and Broderick Street, the separated bikeway becomes a "mixing zone" and right-turning motor vehicles cross the bike lane to complete their turns. The mixing zone at this intersection uses white bicycle markings on a green background. There is also a "bike box" at the intersection. (See picture below).

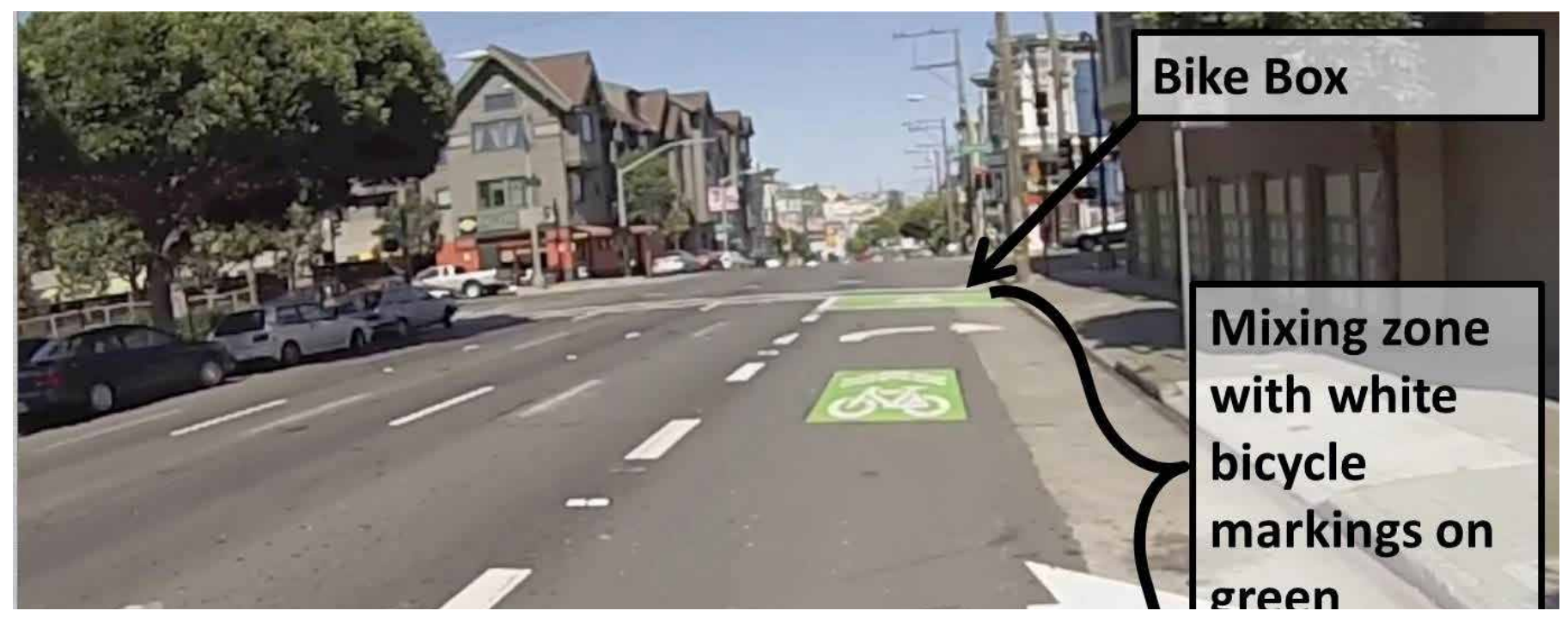




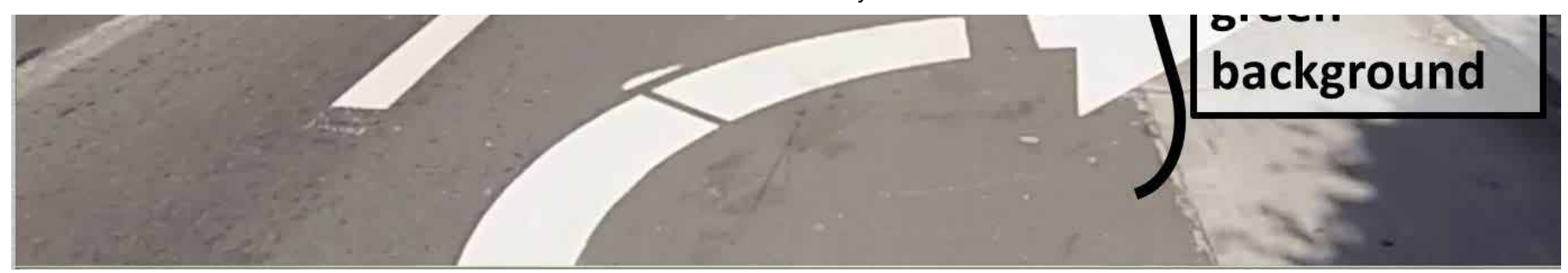

Indicate your level of agreement with the following statements

\begin{tabular}{|c|c|c|c|c|c|}
\hline & Strongly Disagree & $\begin{array}{l}\text { Somewhat } \\
\text { Disagree }\end{array}$ & Somewhat Agree & Strongly Agree & No Opinion \\
\hline $\begin{array}{l}\text { I understand where I am supposed to ride when } \\
\text { approaching the intersection. }\end{array}$ & O & 0 & 0 & 0 & O \\
\hline $\begin{array}{l}\text { Turning motorists generally yield to bicyclists when } \\
\text { moving through the mixing zone to take a right turn. }\end{array}$ & 0 & 0 & 0 & 0 & 0 \\
\hline $\begin{array}{r}\text { Motorists generally understand how to make right turns } \\
\text { at these intersections. }\end{array}$ & 0 & 0 & 0 & 0 & O \\
\hline Cars rarely block my pathway through the mixing zone. & 0 & 0 & 0 & 0 & 0 \\
\hline $\begin{array}{l}\text { I often see motorists making right turns from the wrong } \\
\text { lane (i.e. the lanes to the left of the mixing zone rather } \\
\text { than the right-turn lane). }\end{array}$ & 0 & 0 & 0 & 0 & O \\
\hline $\begin{array}{r}\text { I generally feel safe when bicycling through the mixing } \\
\text { zone. }\end{array}$ & 0 & 0 & O & 0 & O \\
\hline $\begin{array}{r}\text { When the traffic light is red, I use the bike box to wait } \\
\text { for a green light. }\end{array}$ & 0 & 0 & 0 & 0 & 0 \\
\hline $\begin{array}{l}\text { It is easy for me to make my way into the bike box } \\
\text { when the traffic light is red and there are cars stopped } \\
\text { in the right-turn lane in front of me. }\end{array}$ & 0 & 0 & 0 & ○ & 0 \\
\hline
\end{tabular}

\section{Bicycle Signal: Intersection of Oak Street and Broderick Street}

As you travel east on Oak Street (toward downtown), a new bicycle signal has been installed at the intersection of Oak Street and Broderick Street (see picture below). Previously, there was no bike signal. Now there are bicycle signals on the near and far side of the intersection with Broderick Street.

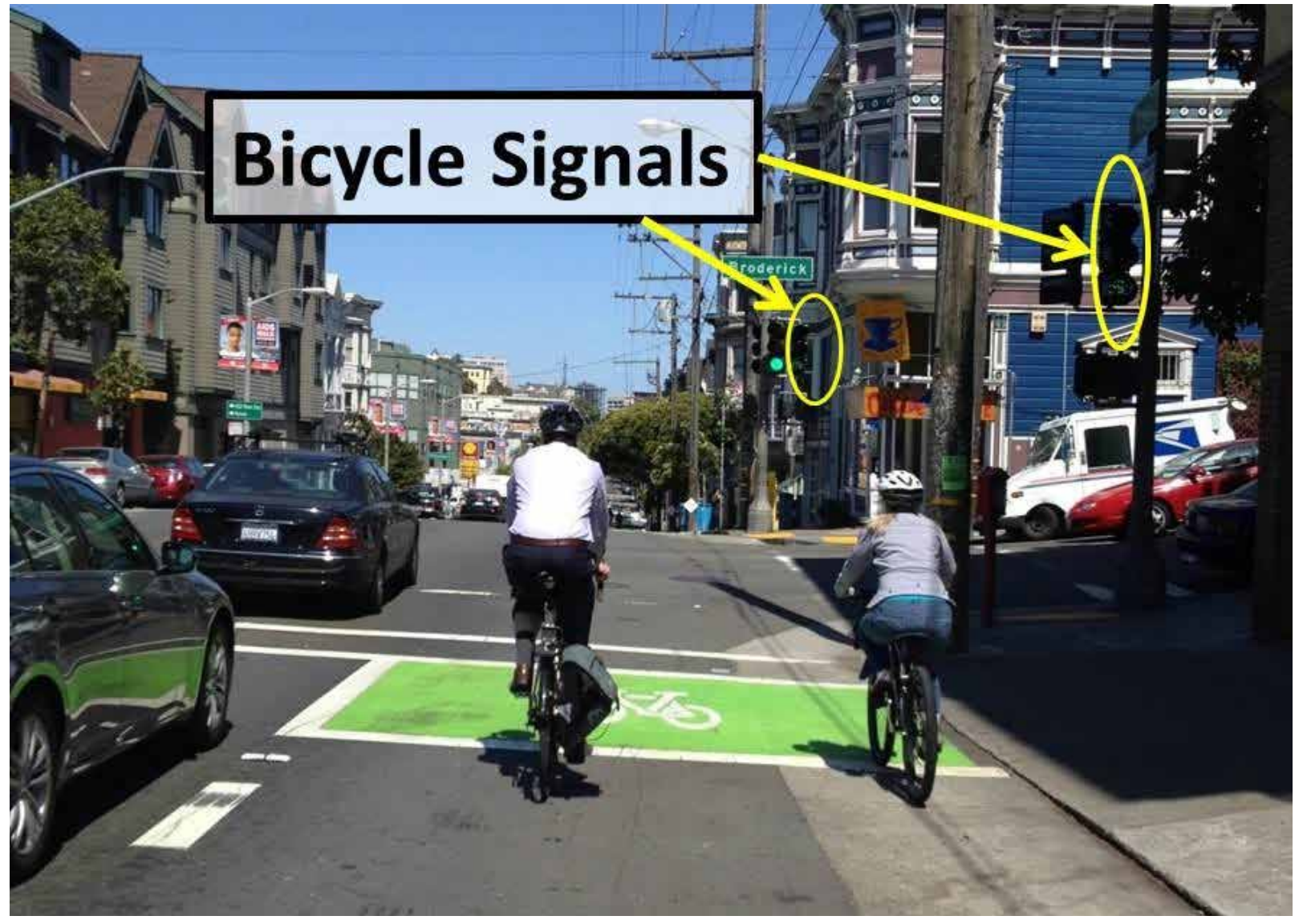




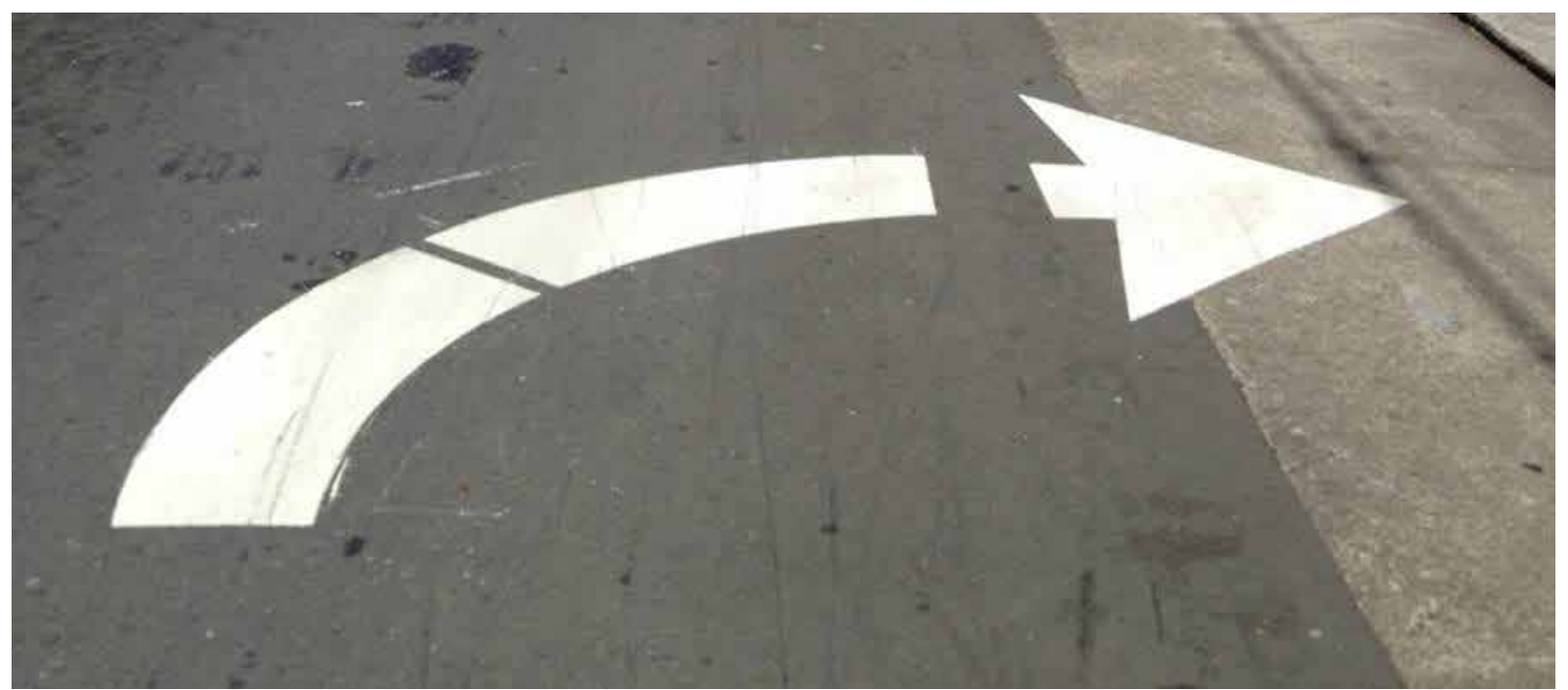

Have you bicycled through this intersection travelling east since the bicycle signal was installed?
Yes
No
I have never noticed the bike signal

When riding on Oak Street, do you most often

Follow the standard traffic signals (stop on the red, go on the green)

○

Follow the bicycle signals (stop on bike red, go on bike green)

Other (please explain)

Because of the changes to this intersection:

\begin{tabular}{|c|c|c|c|c|c|c|}
\hline & Decreased a Lot & $\begin{array}{l}\text { Decreased } \\
\text { Somewhat }\end{array}$ & Not Changed & $\begin{array}{l}\text { Increased } \\
\text { Somewhat }\end{array}$ & Increased a Lot & No Opinion \\
\hline $\begin{array}{r}\text { how safe I feel bicycling through this } \\
\text { intersection has ... }\end{array}$ & 0 & 0 & 0 & 0 & 0 & 0 \\
\hline $\begin{array}{r}\text { how stressful bicycling through this } \\
\text { intersection is has . . }\end{array}$ & 0 & 0 & 0 & 0 & 0 & 0 \\
\hline $\begin{array}{l}\text { how long I have to wait for a green signal to } \\
\text { get through the intersection has ... }\end{array}$ & 0 & 0 & 0 & 0 & 0 & 0 \\
\hline $\begin{array}{r}\text { how likely I am to wait for the signal to turn } \\
\text { green has ... }\end{array}$ & 0 & 0 & 0 & 0 & 0 & 0 \\
\hline
\end{tabular}

How comfortable do you feel bicycling through this intersection?
(1) Very Uncomfortable
(2)
(3)
(4)
(5)
(6) Very Comfortable

Do you ride through this intersection differently now than you did before the changes were made?
Yes
No 
How have you changed how you ride through the intersection?

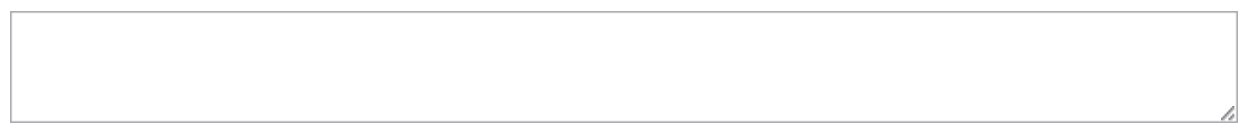

Consider when you are traveling the whole length of Oak Street between Baker Street and Scott Street.

\begin{tabular}{c|c} 
& become easier \\
\hline $\begin{array}{c}\text { Since the installation of the bicycle } \\
\text { signal, hitting most or all green } \\
\text { lights on Oak Street has ... }\end{array}$ & 0
\end{tabular}

\section{Left Turns}

When you bicycle on the Oak Street separated bikeway, do you ever make left turns off of Oak Street?

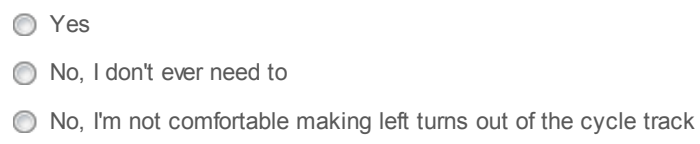

How do you make your left turn from the separated bikeway?

I leave the separated bikeway before the intersection and ride over to the left lane to make my turn

I ride to the intersection and cross in the crosswalk when the light is red

Other (please describe)

\section{About What You Encounter in the Separated Bikeway}

Please indicate how often you have observed the following on your rides in the Oak Street separated bikeway, AND whether you think this is a problem that needs to be addressed

\begin{tabular}{|c|c|c|c|c|c|c|c|}
\hline & \multicolumn{4}{|c|}{$\begin{array}{l}\text { How often do you encounter the following } \\
\underline{\text { IN the Protected Bikeway? }}\end{array}$} & \multicolumn{3}{|c|}{ How much of a problem is this? } \\
\hline & Never & Rarely & Sometimes & Often & $\begin{array}{c}\text { Not a } \\
\text { problem }\end{array}$ & $\begin{array}{l}\text { Minor } \\
\text { problem }\end{array}$ & $\begin{array}{c}\text { Major } \\
\text { Problem }\end{array}$ \\
\hline Cars parking & 0 & 0 & 0 & 0 & 0 & 0 & 0 \\
\hline $\begin{array}{l}\text { Cars loading or } \\
\text { unloading passengers }\end{array}$ & 0 & 0 & 0 & 0 & $\bigcirc$ & 0 & 0 \\
\hline $\begin{array}{l}\text { Delivery vehicles } \\
\text { loading or unloading }\end{array}$ & 0 & 0 & 0 & 0 & 0 & 0 & 0 \\
\hline Taxis & $\bigcirc$ & 0 & 0 & $\bigcirc$ & $\bigcirc$ & $\bigcirc$ & $\bigcirc$ \\
\hline $\begin{array}{r}\text { Cars/trucks driving } \\
\text { where they are not } \\
\text { supposed to (in the } \\
\text { bikeway) }\end{array}$ & 0 & 0 & 0 & 0 & 0 & 0 & 0 \\
\hline $\begin{array}{r}\text { Cars/trucks waiting to } \\
\text { make turns OFF of } \\
\text { Oak Street }\end{array}$ & 0 & 0 & 0 & 0 & 0 & O & 0 \\
\hline $\begin{array}{r}\text { Cars/trucks waiting to } \\
\text { pull out ONTO Oak } \\
\text { Street }\end{array}$ & 0 & 0 & 0 & 0 & 0 & 0 & 0 \\
\hline $\begin{array}{r}\text { People walking in the } \\
\text { bikeway }\end{array}$ & 0 & 0 & 0 & 0 & 0 & 0 & 0 \\
\hline $\begin{array}{l}\text { People standing in } \\
\text { the bikewav while }\end{array}$ & - & - & - & - & - & - & - \\
\hline
\end{tabular}


waiting to cross the street

Bicyclists traveling in the WRONG direction

00

O

○

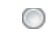

○

○

$0 \quad 0 \quad 0$

While riding in the Oak Street separated bikeway, have you had collisions or near-collisions with other people or objects on the road?

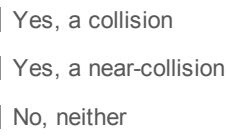

If you have been involved in a collision or near-collision, please indicate what other people or objects were involved.

Check all that apply.

\begin{tabular}{|c|c|c|}
\hline & Collision & Near Collision \\
\hline Another bicyclist & $\square$ & $\square$ \\
\hline A pedestrian & $\square$ & $\square$ \\
\hline A turning car & $\square$ & $\square$ \\
\hline A parking car & $\square$ & $\square$ \\
\hline A delivery truck & $\square$ & $\square$ \\
\hline A bus & $\square$ & $\square$ \\
\hline A taxi & $\square$ & $\square$ \\
\hline One of the plastic flexposts & $\square$ & $\square$ \\
\hline $\begin{array}{r}\text { Other stationary object (please } \\
\text { describe). }\end{array}$ & $\square$ & $\square$ \\
\hline $\begin{array}{l}\text { Something else (please } \\
\text { describe). }\end{array}$ & $\square$ & $\square$ \\
\hline
\end{tabular}

Please provide a brief description of the collision(s) you were involved in while riding a bicycle in the bikeway.

\section{Fell Street Separated Bikeway}

A separated bikeway, also known as a "cycle track", was recently built on the left side of Fell Street between Scott Street and Baker Street. Bike lanes are separated from other traffic lanes by a combination of a striped "buffer" zone and plastic "flexposts" (see picture below). 

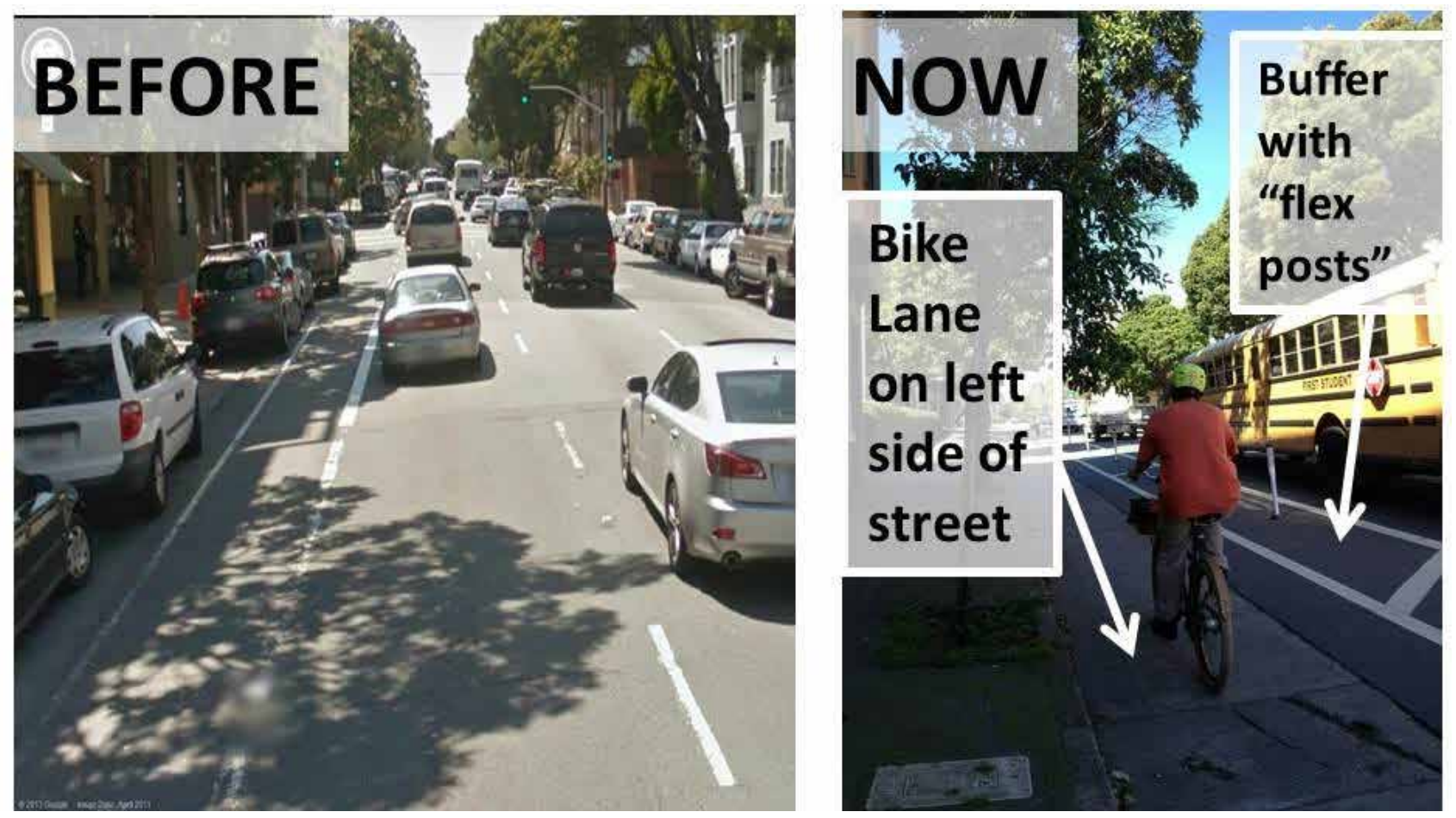

The next set of questions are about the Fell Street separated bikeway.

Have you ridden a bicycle in the Fell Street separated bikeway since it was installed?

Yes

No

How often do you ride a bicycle on this part of Fell Street?
This was my first time
Less than once a month
A few times a month
A few times a week
Daily

How comfortable do you feel when bicycling in the Fell Street separated bikeway?
(1) Very Uncomfortable
(2)
(3)
(4)
(5)
(6) Very Comfortable

Since the Fell Street separated bikeway was built, do you travel on this route:
Less frequently
More frequently
- . 
When bicycling on Fell Street between Scott Street and Baker Street, how often you ride on the following:

\begin{tabular}{|c|c|c|c|c|}
\hline & Always & Most of the time & Some of the time & Never \\
\hline Separated bikeway & 0 & 0 & 0 & 0 \\
\hline Standard traffic lanes & 0 & $\bigcirc$ & $\bigcirc$ & 0 \\
\hline In the gutter area, near the curb & 0 & $\bigcirc$ & 0 & 0 \\
\hline Sidewalk & 0 & 0 & $\bigcirc$ & $\bigcirc$ \\
\hline
\end{tabular}

Consider the trip you were making when you were handed the postcard. Before the Fell Street separated bikeway was built, how would you have made this trip?
By bicycle, using this same route
By bicycle, using another route - (Please specify which route):
By foot
O
By public transportation
By car
By taxi
I would NOT have taken this trip
Other (please specify below)

\section{About Changes due to the Separated Bikeway}

Because of the Fell Street separated bikeway ...

\begin{tabular}{r|c|c|} 
I feel the safety of bicycling on Fell Street has . . & $\begin{array}{c}\text { Decreased a } \\
\text { Lot }\end{array}$. \\
the time it takes me to bicycle on Fell Street has . . . \\
Somewhat \\
the usefulness of Fell Street for getting to places I want to go has . . .
\end{tabular}

\section{Your Opinions About the Separated Bikeway}

Please indicate if you agree or disagree with the following statements about the Fell Street separated bikeway:

$\begin{array}{r}\text { I would go out of my way to ride on Fell Street compared } \\ \text { to other streets. }\end{array}$
Taking Fell Street is the most direct bicycle route to my
destination.
The buffer with the striped buffer and plastic flexposts
between the traffic lanes and the bikeway makes me feel
safe.




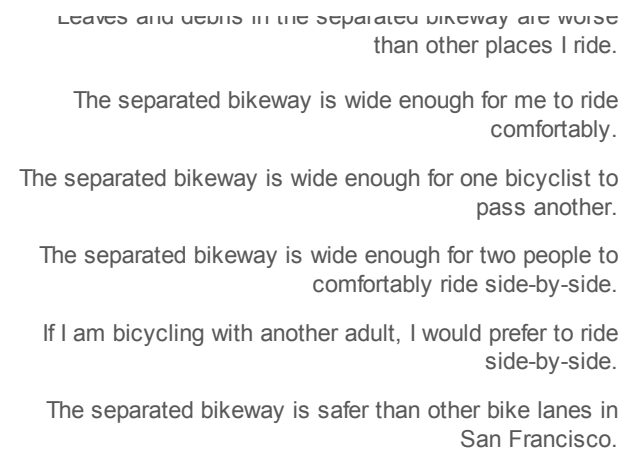

than other places I ride.
theu ninevray aic vruise

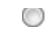

San Francisco.

○

0

○

○

$\bigcirc$

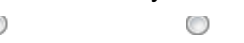

0

$0 \quad 0$

0

0

0

0

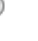

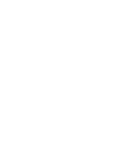

0

00

0

- 0

0

○

○

○

0

○

(1)

$+2$

Please indicate if you disagree or agree with the following statements about the effectiveness of the Fell Street separated bikeway:

\begin{tabular}{|c|c|c|c|c|c|}
\hline & $\begin{array}{l}\text { Strongly } \\
\text { Disagree }\end{array}$ & $\begin{array}{l}\text { Somewhat } \\
\text { Disagree }\end{array}$ & Somewhat Agree & Strongly Agree & No Opinion \\
\hline $\begin{array}{l}\text { The separated bikeway makes it clear where cars can be } \\
\text { and where the designated bicycle lanes are. }\end{array}$ & 0 & 0 & 0 & 0 & 0 \\
\hline $\begin{array}{l}\text { The intersection signals, signs, and street markings } \\
\text { make it clear who has the right-of-way at intersections. }\end{array}$ & 0 & 0 & 0 & 0 & 0 \\
\hline The buffer effectively separates bikes from cars. & 0 & 0 & 0 & 0 & 0 \\
\hline The buffer does a good job at protecting bikes from cars. & 0 & 0 & 0 & 0 & 0 \\
\hline $\begin{array}{r}\text { The separated bikeway makes drivers and bicyclists } \\
\text { more predictable. }\end{array}$ & 0 & 0 & 0 & 0 & 0 \\
\hline $\begin{array}{r}\text { The separated bikeway makes it clear where bicyclists } \\
\text { and pedestrians should be. }\end{array}$ & 0 & 0 & 0 & 0 & 0 \\
\hline $\begin{array}{l}\text { The separated bikeways' design effectively separates } \\
\text { bicyclists from pedestrians. }\end{array}$ & 0 & 0 & 0 & 0 & 0 \\
\hline
\end{tabular}

\section{Intersection of Fell Street and Divisadero Street}

At the intersection of Fell Street and Divisadero Street, the separated bikeway shifts out toward the motor vehicle traffic lane and left-turning motor vehicles cross the bike lane to complete their turns. This area is called a "mixing zone" (See picture below). Sometimes the green-marked bike lane is called an "advisory lane."

The second photo is the same intersection, closer to the intersection.

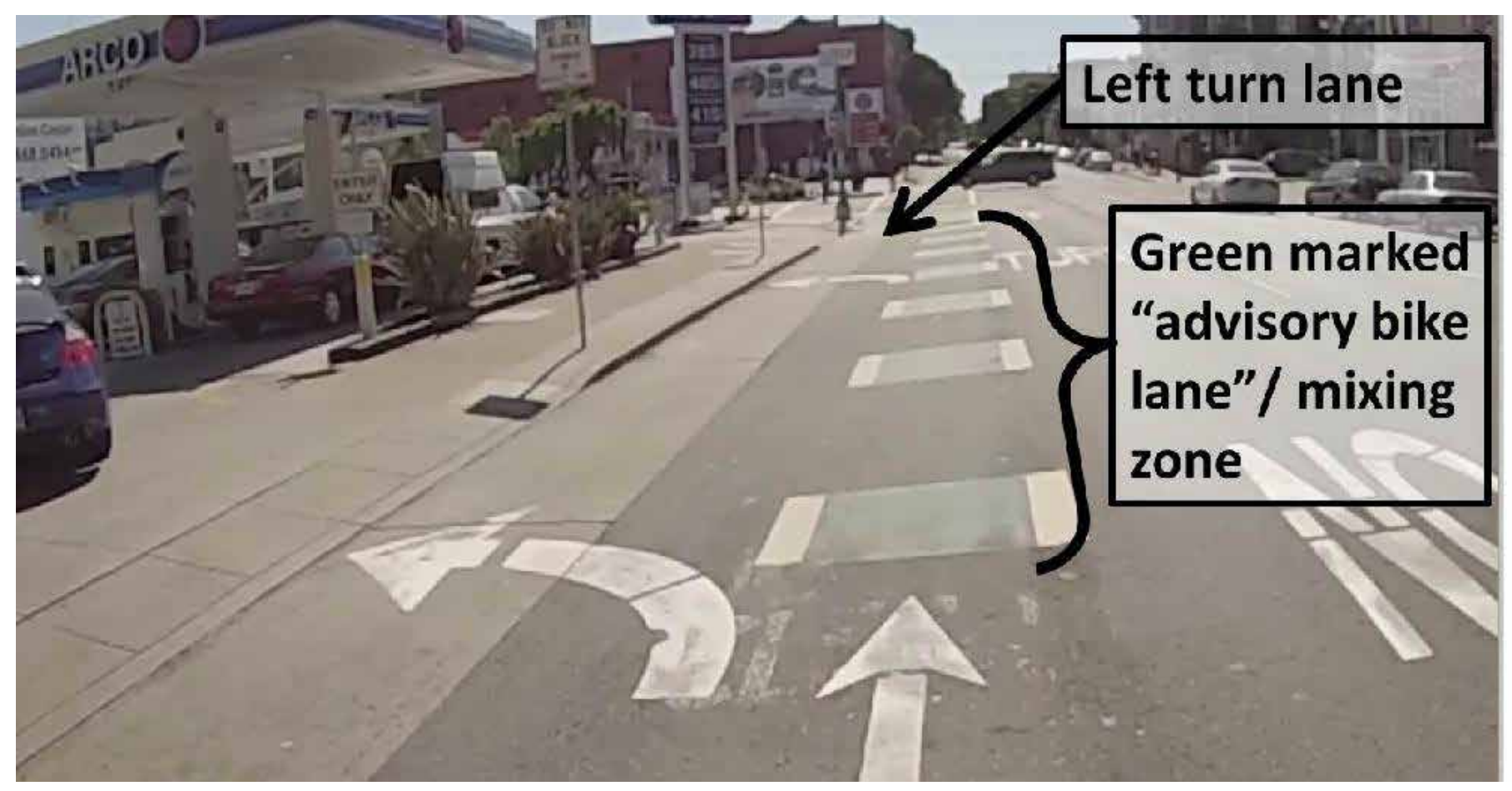




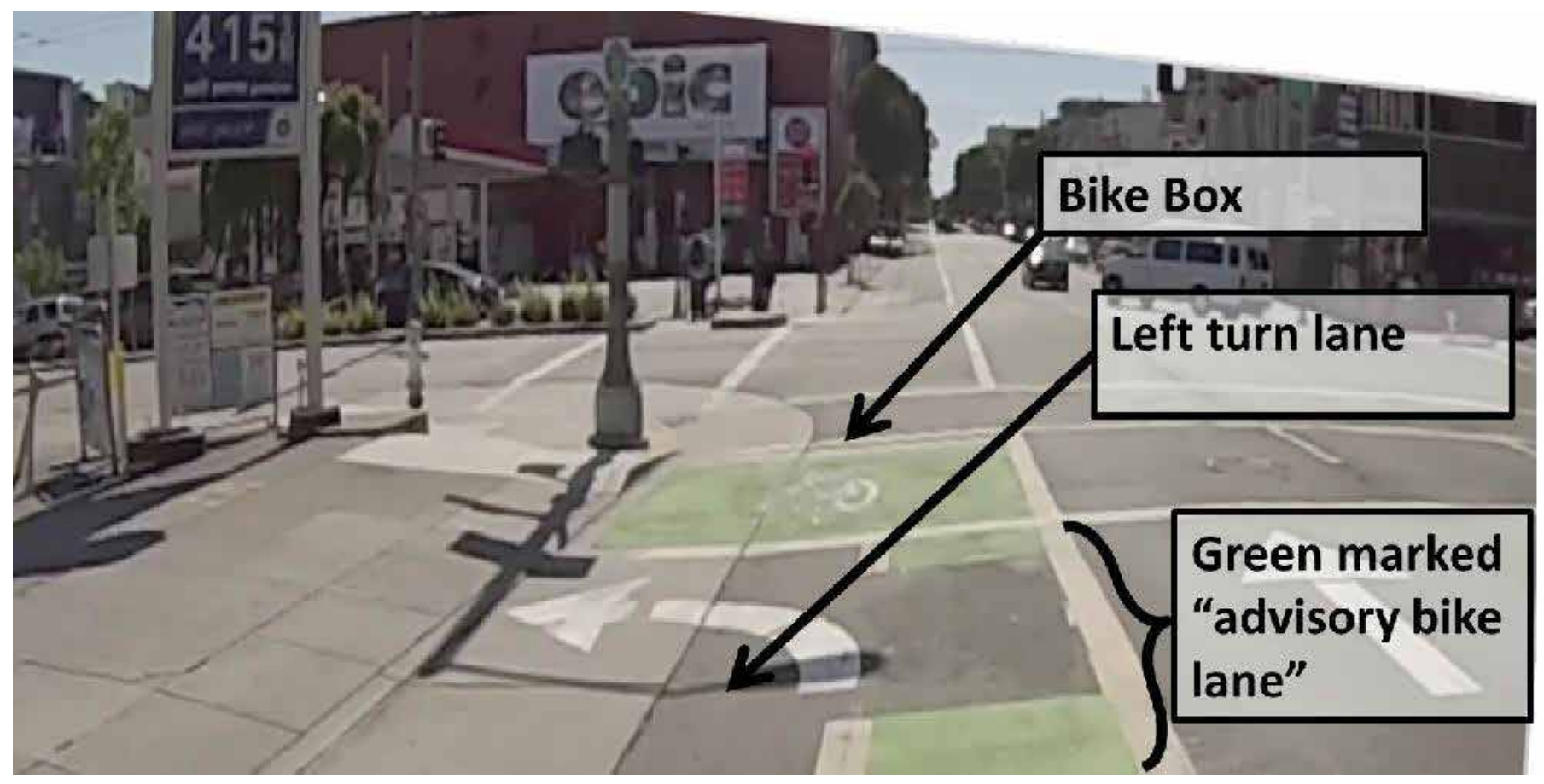

$\underline{\text { Indicate your level of agreement with the following statements }}$

$\begin{gathered}\text { I understand where I am supposed to ride when } \\ \text { approaching the intersection. }\end{gathered}$
$\begin{gathered}\text { I usually follow the bicycle lane marking and move over } \\ \text { to the right (into the green marked bike lane) when } \\ \text { approaching the intersection. }\end{gathered}$
Turning motorists generally yield to bicyclists when
moving through the mixing zone and into the left-turn
lane.




\section{Intersection of Fell Street and Broderick Street}

At the intersection of Fell Street and Broderick Street, the separated bikeway becomes a "mixing zone" for bicycles and left-turning motor vehicles. The mixing zone at this intersection uses green painted bars across the lane (See picture below).

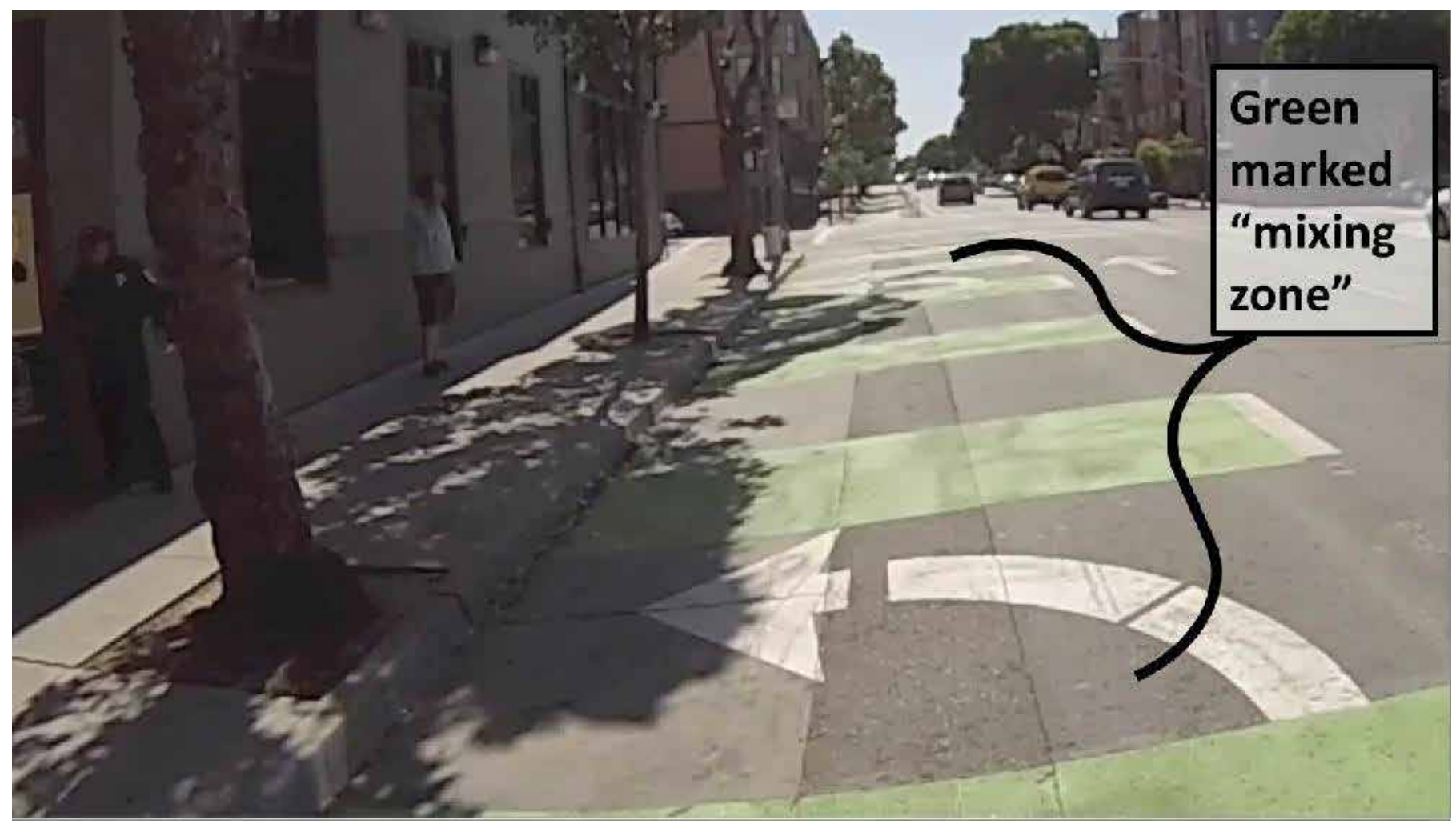

\section{$\underline{\text { Indicate your level of agreement with the following statements }}$}

\begin{tabular}{|c|c|c|c|c|c|}
\hline & Strongly Disagree & $\begin{array}{l}\text { Somewhat } \\
\text { Disagree }\end{array}$ & Somewhat Agree & Strongly Agree & No Opinion \\
\hline $\begin{array}{r}\text { I understand where I am supposed to ride when } \\
\text { approaching the intersection. }\end{array}$ & 0 & O & O & 0 & 0 \\
\hline $\begin{array}{l}\text { Turning motorists generally yield to bicyclists when } \\
\text { moving through the mixing zone to take a left turn. }\end{array}$ & 0 & 0 & 0 & O & 0 \\
\hline $\begin{array}{r}\text { Motorists generally understand how to make left turns } \\
\text { at these intersections. }\end{array}$ & ○ & 0 & O & O & 0 \\
\hline Cars rarely block my pathway through the mixing zone. & O & 0 & O & 0 & 0 \\
\hline $\begin{array}{l}\text { I often see motorists making left turns from the wrong } \\
\text { lane (i.e. the lanes to the right of the mixing zone rather } \\
\text { than the lane with the mixing zone). }\end{array}$ & O & O & 0 & O & O \\
\hline $\begin{array}{r}\text { I generally feel safe when bicycling through the mixing } \\
\text { zone. }\end{array}$ & 0 & O & 0 & 0 & 0 \\
\hline
\end{tabular}

\section{Right Turns}

When you bicycle on the Fell Street separated bikeway, do you ever make right turns off of Fell Street?
Yes
No, I don't ever need to
No, I'm not comfortable making left turns out of the cycle track 
How do you make your right turn from the separated bikeway?

I leave the separated bikeway before the intersection and ride over to the right lane to make my turn

I ride to the intersection and cross in the crosswalk when the light is red

Other (please describe)

\section{About What You Encounter in the Cycle Track}

Please indicate how often you have observed the following on your rides in the Fell Street separated bikeway, AND whether you think this is a problem that needs to be addressed

\begin{tabular}{|c|c|c|c|c|c|c|c|}
\hline & \multicolumn{4}{|c|}{$\begin{array}{l}\text { How often do you encounter the following } \\
\qquad \text { IN the Protected Bikeway? }\end{array}$} & \multicolumn{3}{|c|}{ How much of a problem is this? } \\
\hline & Never & Rarely & Sometimes & Often & $\begin{array}{c}\text { Not a } \\
\text { problem }\end{array}$ & $\begin{array}{c}\text { Minor } \\
\text { problem }\end{array}$ & $\begin{array}{c}\text { Major } \\
\text { Problem }\end{array}$ \\
\hline Cars parking & 0 & 0 & 0 & 0 & 0 & 0 & 0 \\
\hline $\begin{array}{l}\text { Cars loading or } \\
\text { unloading passengers }\end{array}$ & 0 & 0 & 0 & 0 & 0 & 0 & 0 \\
\hline $\begin{array}{l}\text { Delivery vehicles } \\
\text { loading or unloading }\end{array}$ & 0 & 0 & 0 & 0 & 0 & 0 & 0 \\
\hline Taxis & 0 & 0 & 0 & 0 & 0 & 0 & 0 \\
\hline $\begin{array}{r}\text { Cars/trucks driving } \\
\text { where they are not } \\
\text { supposed to (in the } \\
\text { bikeway) }\end{array}$ & 0 & 0 & 0 & 0 & $\bigcirc$ & 0 & 0 \\
\hline $\begin{array}{r}\text { Cars/trucks waiting to } \\
\text { make turns OFF of } \\
\text { Fell Street }\end{array}$ & 0 & 0 & 0 & 0 & 0 & 0 & 0 \\
\hline $\begin{array}{r}\text { Cars/trucks waiting to } \\
\text { pull out ONTO Fell } \\
\text { Street }\end{array}$ & 0 & 0 & 0 & 0 & 0 & ○ & 0 \\
\hline $\begin{array}{r}\text { People walking in the } \\
\text { bikeway }\end{array}$ & 0 & 0 & 0 & 0 & 0 & $\bigcirc$ & 0 \\
\hline $\begin{array}{r}\text { People standing in } \\
\text { the bikeway while } \\
\text { waiting to cross the } \\
\text { street }\end{array}$ & 0 & 0 & 0 & 0 & 0 & 0 & 0 \\
\hline $\begin{array}{l}\text { Bicyclists traveling in } \\
\text { the WRONG direction }\end{array}$ & ○ & O & 0 & 0 & $\bigcirc$ & $\bigcirc$ & 0 \\
\hline
\end{tabular}

While riding in the Fell Street separated bikeway, have you had collisions or near-collisions with other people or objects on the road?
Yes, a collision
Yes, a near-collision
No, neither

If you have been involved in a collision or near-collision, please indicate what other people or objects were involved.

Check all that apply.

\begin{tabular}{r|r|} 
Near Collision & Collision \\
\hline Another bicyclist & $\square$ \\
A pedestrian & $\square$ \\
A turning car & $\square$ \\
A parking car & $\square$ \\
A parked car & $\square$ \\
A delivery truck & $\square$ \\
A bus & $\square$ \\
A taxi & $\square$ \\
\hline
\end{tabular}




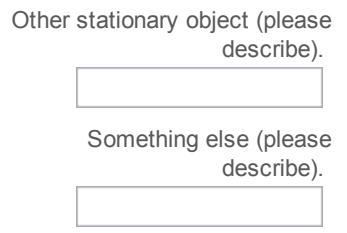

Please provide a brief description of the collision(s) you were involved in while riding a bicycle in the bikeway.

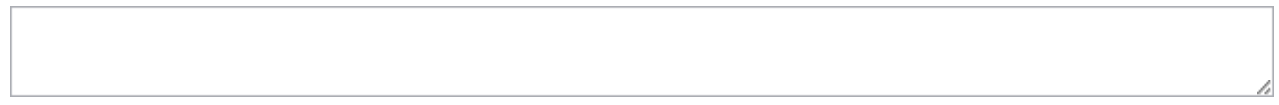

\section{Overall Impressions of the Separated Bikeway}

What is the best thing about the separated bikeway?

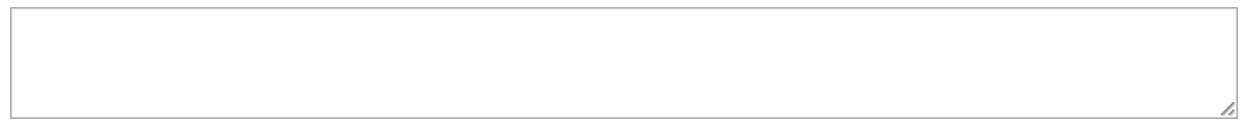

What should be improved about the separated bikeway?

\section{About Bicycling and Comfort}

Regardless of whether you currently bicycle in all the following situations, please consider how comfortable you would be riding a bicycle in each place:
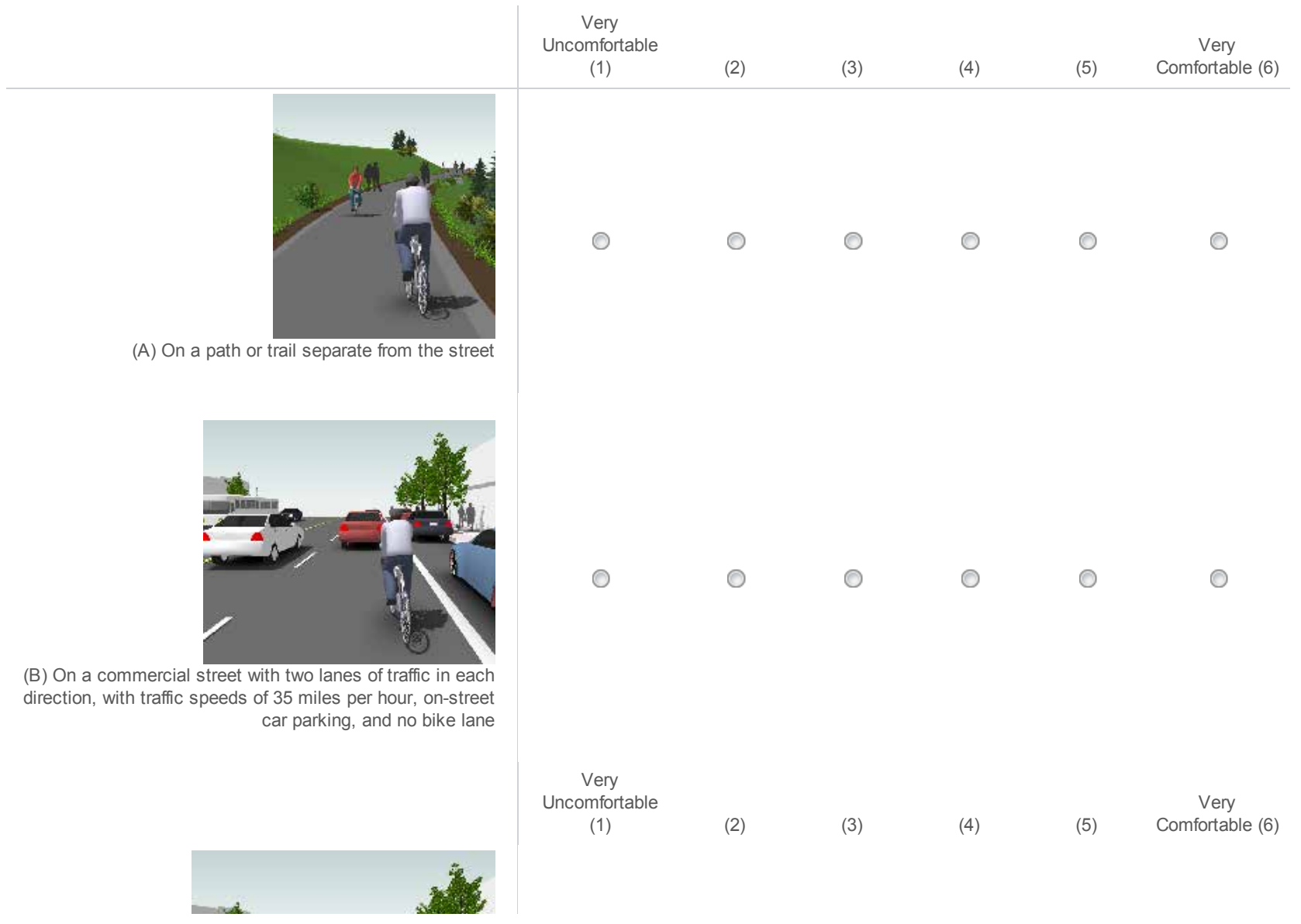
(1)
(2)
(3)
(4)
Comfortable $(6)$ 
(C) On a similar street to (B), but with a striped bike lane

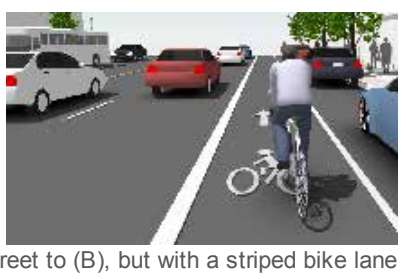

(D) On a similar street to (B), but with a physically separated bike lane

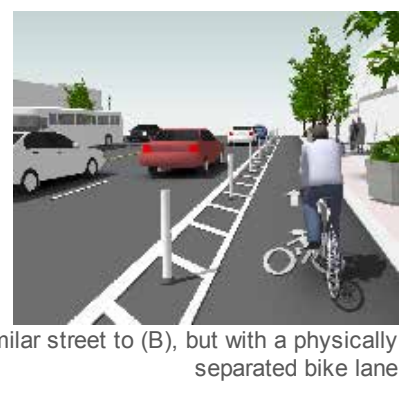

How comfortable would you feel bicycling on a commercial street with two lanes of traffic in each direction, with traffic speeds of 35 miles per hour (Situation D above), but with the following types of separation from traffic:

Very
Uncomfortable

(1)
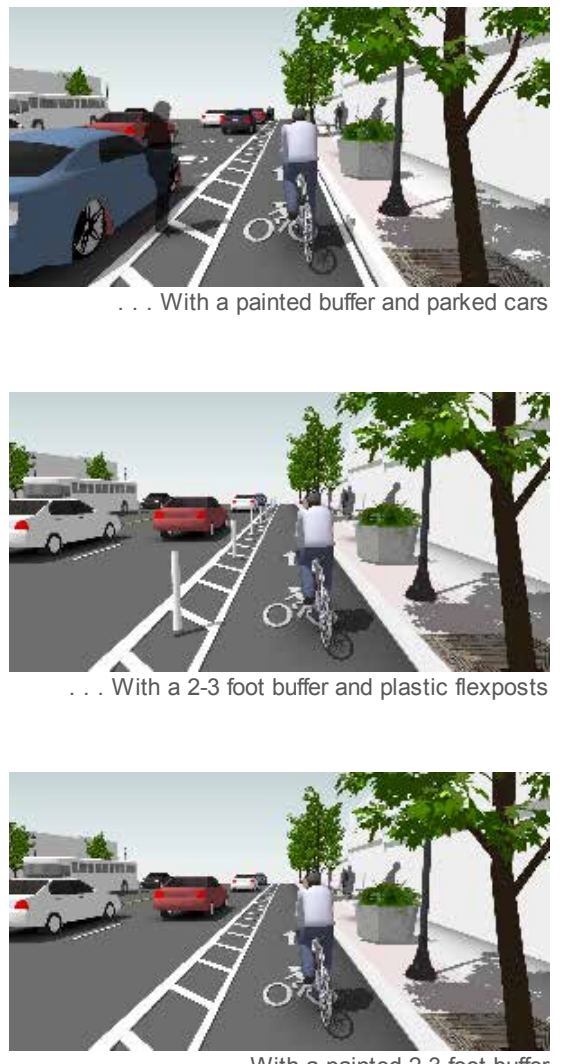

. With a painted 2-3 foot buffer
(2)

(3)

(4)

(5)

Very Comfortable (6) 


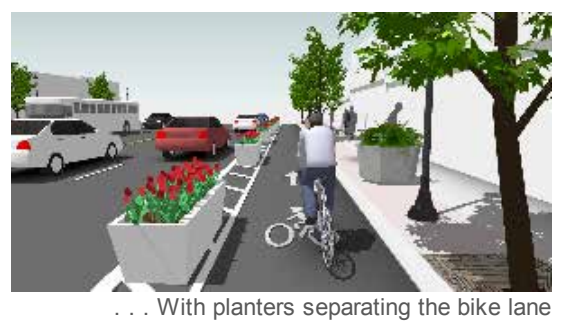

With planters separating the bike lane
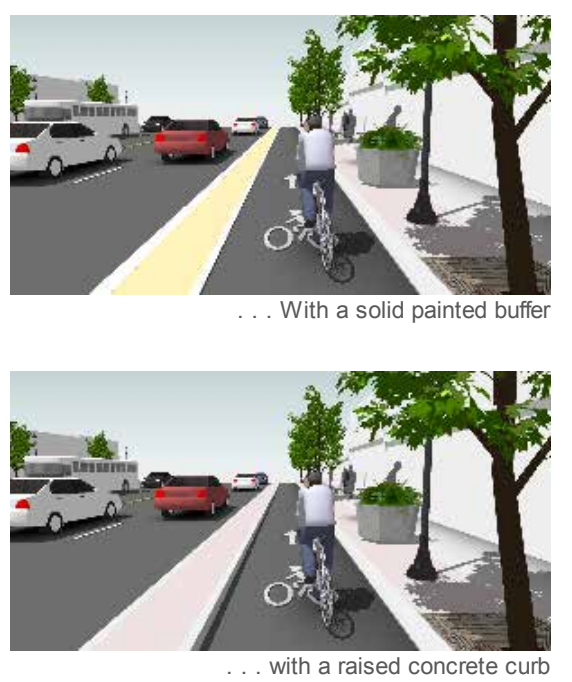

\section{.}

\section{About People you Encounter on the Street}

Please indicate if you agree or disagree with the following statements:

\begin{tabular}{r|ccccc} 
& Strongly Disagree & Somewhat Disagree & Somewhat Agree & Strongly Agree & No Opinion \\
\hline Most drivers follow the rules of the road. & 0 & 0 & 0 & 0 \\
Most drivers are predictable. & 0 & 0 & 0 & 0 \\
Most bicyclists follow the rules of the road. & 0 & 0 & 0 & 0 \\
Most bicyclists are predictable. & 0 & 0 & 0 & 0 \\
Most pedestrians follow the rules of the road. & 0 & 0 & 0 & 0
\end{tabular}

\section{About You}

We have a few questions about you so that we may understand the characteristics of our survey respondents.

Do you have a current:

\begin{tabular}{l|cc} 
& Yes & No \\
\hline Driver's License & 0 & $\bigcirc$ \\
Transit Pass & 0 & 0 \\
Bike-share Membership & $\bigcirc$ & $\bigcirc$ \\
Car-share Membership & 0
\end{tabular}


How many working adult bicycles does your household own?

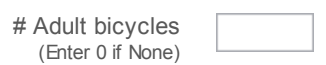

(Enter 0 if None)

INCLUDING YOURSELF, how many people live in your household?

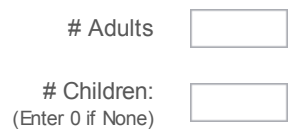

What is your home zip code?

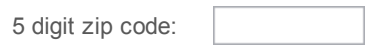

What is your gender?
Male
Female
○

What is your age?

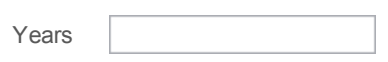

Do you consider yourself: (select all that apply)

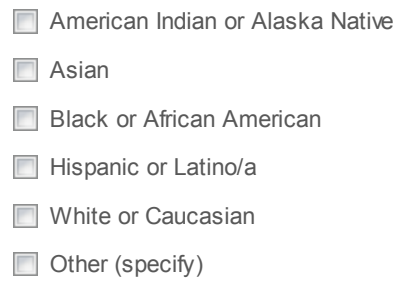

What is your employment status? (Select all that apply)

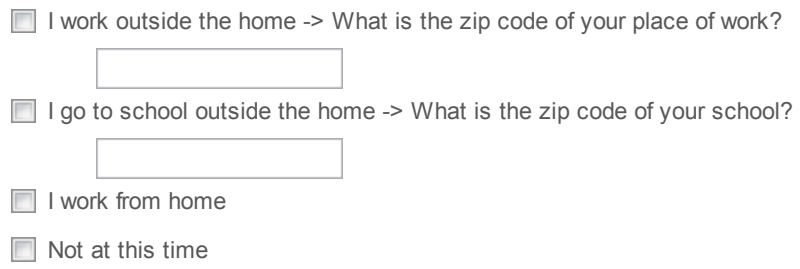

What is your annual household income?

Less than $\$ 25,000$ 

$\$ 25,000$ to less than $\$ 50,000$
$\$ 50,000$ to less than $\$ 75,000$
$\$ 75,000$ to less than $\$ 100,000$
( $\$ 100,000$ to less than $\$ 200,000$
$\$ 200,000$ or more
I prefer not to provide this information

How many years of school have you completed?
Some high school or less
High school diploma or GED
Some College
Trade/Vocational School
○ Associate Degree
Four-year college degree or more
Other (please specify):

Is there anything else that you would like to tell us?

That's all the questions we have. Would you like to be entered into a drawing for one of three $\$ 100$ gift cards to Amazon.com?

(2) Yes, I would like to be entered in the drawing (I'll enter my name on the next page).

No thanks. I will complete my survey by clicking the ">>" button

Enter your name and some way for us to get a hold of you below. We will only use this information to contact you in the event that your entry is selected to receive a gift card. This information will not be associated to any of your survey responses.

Name

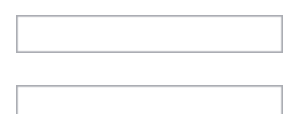

Thanks for taking the survey - please make sure to click the ">>" button to submit your entry and close the survey! 


\section{BICYCLIST: WASHINGTON DC - L STREET}




\section{Neighborhood}

Default Question Block

Dear Bicyclist,

My name is Chris Monsere and I am a faculty member at Portland State University in the Department of Civil and Environmental Engineering. I am the principal investigator on a project researching separated bicycle lanes in six cities across the country.

Hearing from bicyclists like yourse If is a very important part of this study. We are only sampling a select number of bicyclists near the $L$ Street cycle track. Therefore, every response is very important and we hope you will participate. We will share our findings with the District

Department of Transportation and hope that the results will help in future plans for improving bicycling in cities around the United States.

The survey, which starts on the next page, should take about 10-20 minutes. Your participation in the study is voluntary and you may stop taking the survey at any time with no consequences

The postcard that you received has a unique number that only identifies where and when we handed you the postcard. We will protect the confidentiality of your individual survey response. None of your responses will be linked to your name or other identifying personal information .

To say thank you, all people who complete their survey by June 30, 2013 will be entered into a drawing for one of three $\$ 100$ Amazon.com gift cards. You will be asked to enter your name and a way to contact you at the end of the survey - this information is voluntary and will not be connected to your survey data.

If you have concerns or problems about your participation in this study or your rights as a research subject, please contact the Human Subjects Research Review Committee, Office of Research and Strategic Partnerships, Market Center Building Suite 620, Portland State University, (877480-4400). If you have questions about the study itself, please contact our research team directly at streets@pdx.edu or 503-725-2875.

Sincerely,

Christopher M. Monsere, Ph.D., P.E., Associate Professor \& Associate Chair Civil and Environmental Engineering Portland State University

Do you agree to participate in this survey?

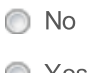

Yes

When you received the postcard for this survey, where were you ...

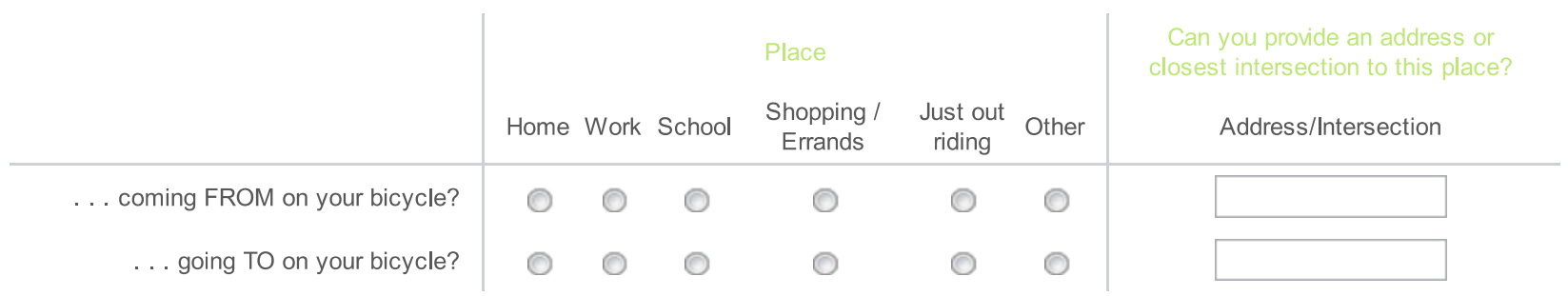

If you selected other, please explain: 
What type of bicycle were you riding when you received the postcard?
Personal Bicycle
Bike Share Bicycle
Rental Bicycle
Other (please specify):

\section{$\underline{\text { L Street Cycle Track }}$}

A separated eastbound bikeway, also known as a "cycle track," was recently built on L Street NW from New Hampshire Avenue to 12th Street. In most places, a standard traffic lane was converted to a bicycle lane with a painted "buffer" area and plastic "flexposts" separating the bike lane from the motor vehicle traffic lanes (see pictures below). The following questions are related to this cycle track.

L Street before and now:

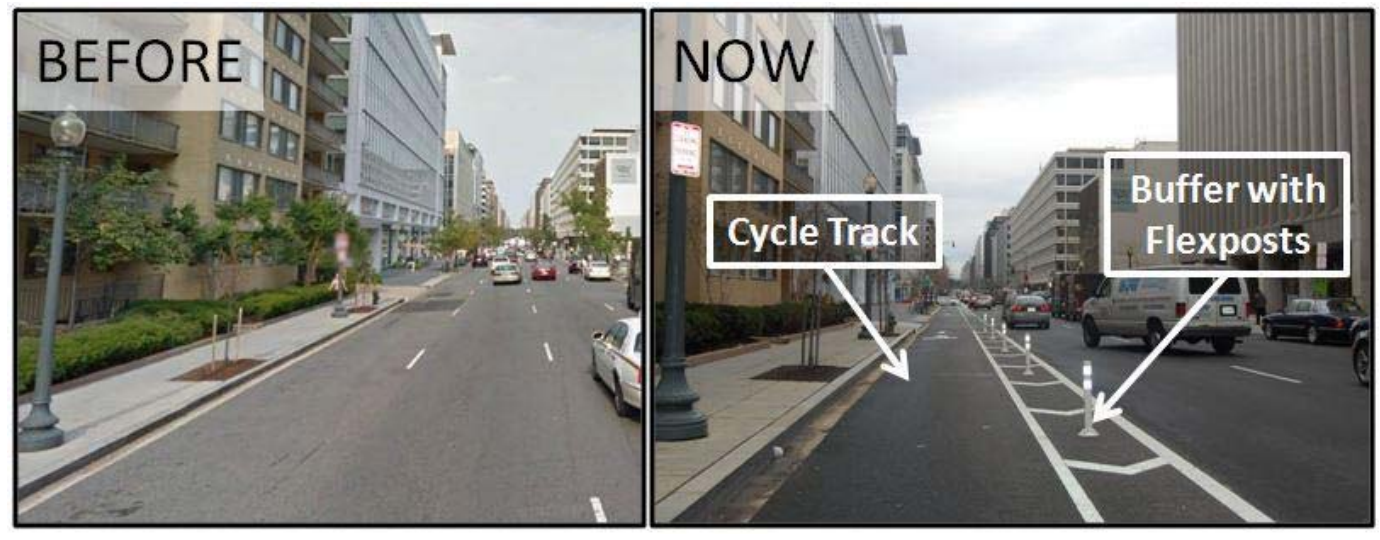

Extent of $L$ Street cycle track

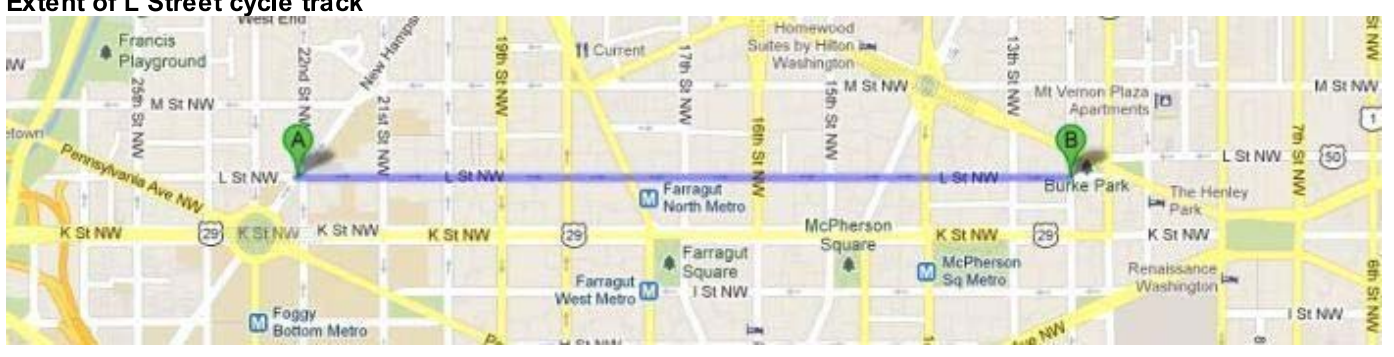

How often do you ride a bicycle on this part of $L$ Street?

This was my first time

Less than once a month

A few times a month

A few times a week

Daily 
How comfortable do you feel when bicycling in the L Street cycle track?
(1) Very Uncomfortable
(2)
(3)
(4)
(5)
(6) Very Comfortable

Since the L Street cycle track was built, do you travel on this route:
Less frequently
More frequently
About the same
This is my first time on this route

When bicycling on L Street between New Hampshire Avenue and Massachusetts Avenue, how often do you ride on the following:

\begin{tabular}{l|cccc} 
& Always & Most of the time & Some of the time & Never \\
\hline $\begin{array}{l}\text { Cycle track, including the mixing } \\
\text { area at intersections }\end{array}$ & 0 & 0 & 0 \\
Standard traffic lanes & 0 & 0 & 0 \\
Curb Area & 0 & 0 & 0
\end{tabular}

Consider the trip you were making when you were handed the postcard. Before the L Street cycle track was built, how would you have made this trip?
By bicycle, using this same route
By bicycle, using another route - (Please specify which route)
By foot
By public transportation
By car
By taxi
I I would NOT have taken this trip
Other (please specify below)

\section{About Changes due to the Cycle Track}

Because of the $\mathbf{L}$ Street cycle track...

\begin{tabular}{|c|c|c|c|c|c|c|}
\hline & $\begin{array}{c}\text { Decreased } \\
\text { a Lot }\end{array}$ & $\begin{array}{l}\text { Decreased } \\
\text { Somewhat }\end{array}$ & $\begin{array}{c}\text { Not } \\
\text { Changed }\end{array}$ & $\begin{array}{l}\text { Increased } \\
\text { Somewhat }\end{array}$ & $\begin{array}{c}\text { Increased } \\
\text { a Lot }\end{array}$ & $\begin{array}{c}\text { No } \\
\text { Opinion }\end{array}$ \\
\hline I feel the safety of bicycling on $L$ Street has ... & ○ & O & ○ & ○ & O & ○ \\
\hline the time it takes me to bicycle on $L$ Street has... & ○ & ○ & ○ & ○ & ○ & O \\
\hline the usefulness of $L$ Street for getting to places I want to go has ... & O & O & ○ & ○ & O & ○ \\
\hline how often I stop at shops and businesses on L Street has... & ○ & ○ & ○ & ○ & O & O \\
\hline
\end{tabular}


how comfortable I feel when bicycling on $L$ Street has . . .

drivers' awareness of people biking on L Street has . . .

drivers' speeds on $\mathrm{L}$ Street have ...

how often I ride a bicycle overall has . . .

the difficulty of navigating around turning motor vehicles has . .

the difficulty of navigating around pedestrians has ...

the difficulty of making a right off of $L$ Street while bicycling has . . .

\section{Your Opinions about the Cycle Track}

Please indicate if you agree or disagree with the following statements about the cycle track:

\begin{tabular}{|c|c|c|c|c|c|}
\hline & $\begin{array}{l}\text { Strongly } \\
\text { Disagree }\end{array}$ & $\begin{array}{l}\text { Somewhat } \\
\text { Disagree }\end{array}$ & $\begin{array}{l}\text { Somewhat } \\
\text { Agree }\end{array}$ & $\begin{array}{l}\text { Strongly } \\
\text { Agree }\end{array}$ & No Opinion \\
\hline $\begin{array}{r}\text { I would go out of my way to ride on } \mathrm{L} \text { Street compared to } \\
\text { other streets. }\end{array}$ & 0 & 0 & 0 & ○ & $\bigcirc$ \\
\hline $\begin{array}{r}\text { Taking } L \text { Street is the most direct bicycle route to my } \\
\text { destination. }\end{array}$ & 0 & 0 & 0 & 0 & 0 \\
\hline $\begin{array}{r}\text { When bicycling on L Street, I always ride in the cycle } \\
\text { track. }\end{array}$ & 0 & ○ & ○ & ○ & ○ \\
\hline $\begin{array}{r}\text { The buffer between the traffic lanes and the cycle track } \\
\text { makes me feel safe. }\end{array}$ & 0 & 0 & 0 & ○ & ○ \\
\hline $\begin{array}{r}\text { During the winter, snow is quickly removed from the cycle } \\
\text { track. }\end{array}$ & 0 & ○ & ○ & ○ & 0 \\
\hline $\begin{array}{l}\text { Leaves and debris in the cycle track are worse than other } \\
\text { places I ride. }\end{array}$ & 0 & ○ & ○ & ○ & 0 \\
\hline The cycle track is wide enough for me to ride comfortably. & 0 & ○ & 0 & 0 & 0 \\
\hline $\begin{array}{r}\text { The cycle track is wide enough for one bicyclist to pass } \\
\text { another. }\end{array}$ & 0 & ○ & ○ & ○ & ○ \\
\hline $\begin{array}{l}\text { The cycle track is wide enough for two people to } \\
\text { comfortably ride side-by-side. }\end{array}$ & ○ & ○ & 0 & ○ & ○ \\
\hline $\begin{array}{r}\text { If I am bicycling with another adult, I would prefer to ride } \\
\text { side-by-side. }\end{array}$ & ○ & ○ & 0 & 0 & 0 \\
\hline The cycle track is safer than other bike lanes in DC. & 0 & 0 & 0 & 0 & 0 \\
\hline
\end{tabular}

Please indicate if you disagree or agree with the following statements about the effectiveness of the L Street cycle track:

\begin{tabular}{|c|c|c|c|c|c|}
\hline & $\begin{array}{l}\text { Strongly } \\
\text { Disagree }\end{array}$ & $\begin{array}{l}\text { Somewhat } \\
\text { Disagree }\end{array}$ & $\begin{array}{l}\text { Somewhat } \\
\text { Agree }\end{array}$ & $\begin{array}{l}\text { Strongly } \\
\text { Agree }\end{array}$ & No Opinion \\
\hline $\begin{array}{l}\text { The cycle track makes it clear where cars can be and } \\
\text { where the designated bicycle lanes are. }\end{array}$ & 0 & 0 & 0 & 0 & ○ \\
\hline $\begin{array}{l}\text { The intersection signals, signs, and street markings } \\
\text { make it clear who has the right-of-way at intersections. }\end{array}$ & ○ & 0 & 0 & 0 & 0 \\
\hline $\begin{array}{r}\text { The buffer and flexposts effectively separate bikes from } \\
\text { cars. }\end{array}$ & 0 & 0 & ○ & 0 & ○ \\
\hline $\begin{array}{l}\text { The buffer and flexposts do a good job at protecting bikes } \\
\text { from cars. }\end{array}$ & O & O & 0 & 0 & ○ \\
\hline $\begin{array}{r}\text { The cycle track makes drivers and bicyclists more } \\
\text { predictable. }\end{array}$ & ○ & 0 & ○ & 0 & ○ \\
\hline $\begin{array}{r}\text { The cycle track makes it clear where pedestrians and } \\
\text { bicyclists should be. }\end{array}$ & ○ & 0 & 0 & 0 & ○ \\
\hline $\begin{array}{r}\text { The cycle track design effectively separates bicyclists } \\
\text { from pedestrians. }\end{array}$ & ○ & ○ & O & ○ & ○ \\
\hline
\end{tabular}


Please indicate how often you have observed the following on your rides in the L Street cycle track, AND whether you think this is a problem that needs to be addressed

\begin{tabular}{|c|c|c|c|c|c|c|c|}
\hline & \multicolumn{4}{|c|}{$\begin{array}{l}\text { How often do you encounter the following } \\
\qquad \underline{\mathbb{N} \text { the CYCLE TRACK? }}\end{array}$} & \multicolumn{3}{|c|}{ How much of a problem is this? } \\
\hline & Never & Rarely & Sometimes & Often & $\begin{array}{c}\text { Not a } \\
\text { problem }\end{array}$ & $\begin{array}{l}\text { Minor } \\
\text { problem }\end{array}$ & $\begin{array}{c}\text { Major } \\
\text { Problem }\end{array}$ \\
\hline Cars parking & ○ & ○ & ○ & ○ & O & ○ & ○ \\
\hline $\begin{array}{l}\text { Cars loading or } \\
\text { unloading passengers }\end{array}$ & O & 0 & ○ & O & O & 0 & O \\
\hline $\begin{array}{l}\text { Delivery vehicles } \\
\text { loading or unloading }\end{array}$ & ○ & ○ & ○ & ○ & O & O & O \\
\hline Taxis & ○ & ○ & O & ○ & O & O & O \\
\hline \multirow{2}{*}{$\begin{array}{r}\text { Cars/trucks driving } \\
\text { where they are not } \\
\text { supposed to (in the } \\
\text { cycle track) }\end{array}$} & ○ & ○ & O & $\bigcirc$ & O & ○ & ○ \\
\hline & Never & Rarely & Sometimes & Often & $\begin{array}{c}\text { Not a } \\
\text { problem }\end{array}$ & $\begin{array}{l}\text { Minor } \\
\text { problem }\end{array}$ & $\begin{array}{l}\text { Major } \\
\text { Problem }\end{array}$ \\
\hline $\begin{array}{r}\text { Cars/trucks waiting to } \\
\text { make turns OFF of } L \\
\text { Street }\end{array}$ & O & ○ & O & ○ & O & ○ & ○ \\
\hline $\begin{array}{r}\text { Cars/trucks waiting to } \\
\text { pull out ONTO L } \\
\text { Street }\end{array}$ & ○ & ○ & ○ & ○ & O & ○ & ○ \\
\hline $\begin{array}{r}\text { People walking in the } \\
\text { cycle track }\end{array}$ & O & ○ & O & ○ & 0 & ○ & O \\
\hline $\begin{array}{r}\text { People standing in } \\
\text { the cycle track while } \\
\text { waiting to cross the } \\
\text { street }\end{array}$ & O & ○ & 0 & ○ & 0 & 0 & 0 \\
\hline $\begin{array}{l}\text { Bicyclists traveling in } \\
\text { the WRONG direction }\end{array}$ & 0 & 0 & 0 & 0 & 0 & $\odot$ & 0 \\
\hline
\end{tabular}

While riding on the L Street cycle track, have you ever had collisions or near-collisions with other people or objects on the road?

$$
\begin{aligned}
& \text { Yes, a collision } \\
& \text { Yes, a near-collision } \\
& \text { No, neither }
\end{aligned}
$$

If you have been involved in a collision or near-collision, please indicate what other people or objects were involved?

(Check all that apply)

\begin{tabular}{r|cc} 
& Collision & Near Collision \\
\hline Another bicyclist & $\square$ & $\square$ \\
A pedestrian & $\square$ & $\square$ \\
A parning car & $\square$ & $\square$ \\
A parked car & $\square$ & $\square$ \\
A delivery truck & $\square$ & $\square$ \\
A bus & $\square$ & $\square$ \\
A taxi & $\square$ \\
One of the cycle track plastic \\
flexposts
\end{tabular}


Please provide a brief description of the collision(s) you were involved in while riding a bicycle in the cycle track.

\section{About Intersections}

At intersections along $L$ Street, the cycle track shifts out toward the motor vehicle traffic lane and left-turning motor vehicles are permitted to cross the bike lane to complete their turns. This area is called a "mixing zone" (See picture below).

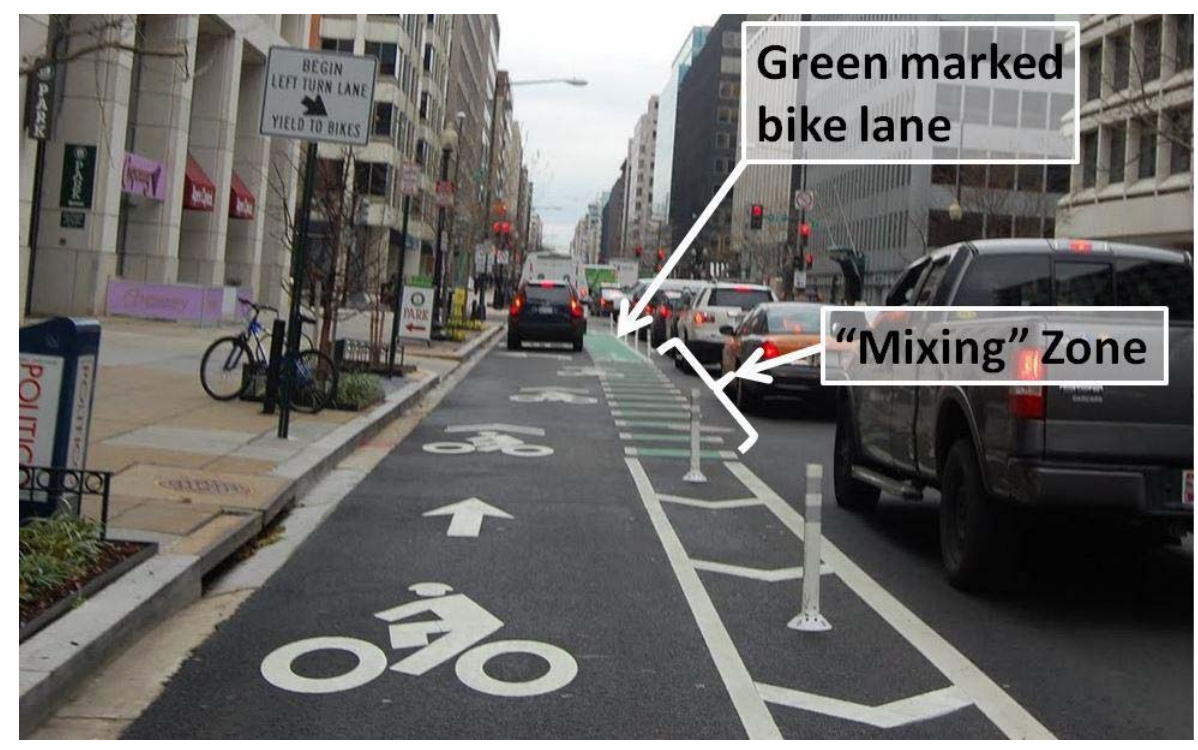

Indicate your level of agreement with the following statements

$\begin{array}{r}\text { I understand where I am supposed to ride when } \\ \text { approaching the intersection. }\end{array}$
$\begin{gathered}\text { I usually follow the bicycle lane marking and move over } \\ \text { to the right (into the green marked bike lane) when } \\ \text { approaching the intersection. }\end{gathered}$
$\begin{gathered}\text { Turning motorists generally yield to bicyclists when } \\ \text { moving through the mixing zone and into the left-turn } \\ \text { lane. }\end{gathered}$
$\begin{gathered}\text { Motorists generally understand how to make left turns } \\ \text { at these intersections. }\end{gathered}$
Cars rarely block my pathway through the mixing zone.
I often see motorists making left turns from the wrong
lane (i.e. the lanes to the right of the cycle track rather
than the left turn lane).


Right Turns:

When you bicycle on L Street, do you ever make right turns off of the cycle track?

Yes

No, I don't ever need to

No, I'm not comfortable making right turns out of the cycle track

At some intersections on L Street, there is a "bike box" area with bike stencils between the "stop bar" and crosswalk (see picture).

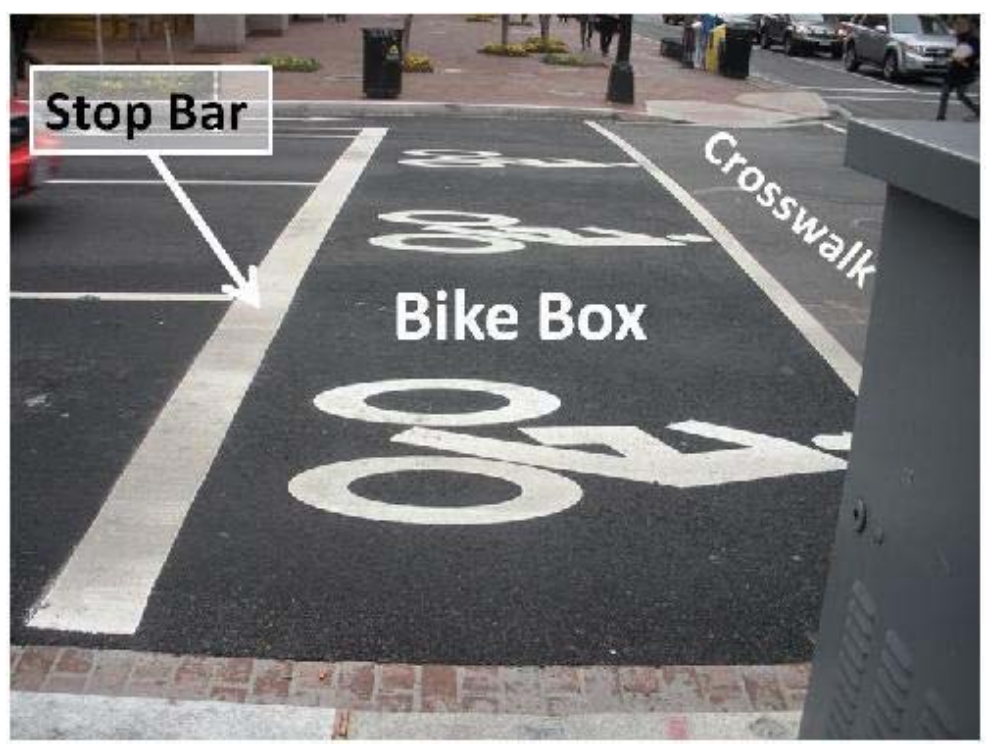

IF you do make right turns off of $L$ Street when bicycling, how do you make right turns? (select all that apply)

When the light is red, and traffic is stopped...

I use the bike box to cross in front of stopped motor vehicles and make my turn.

I use the crosswalk to cross in front of stopped motor vehicles and make my turn.

Prior to the intersection, I move over to the right lane on $L$ Street and complete the turn from there

Other (please describe):

I don't ever make right turns off of $L$ Street

When the light is green, and traffic is moving ...

I wait in the bike lane for a red light or gap in traffic, and then cross to the right side of the street

I move onto the sidewalk and wait for a red light or gap in traffic, and then cross to the right side of the street

Prior to the intersection, I move over to the right lane on $L$ Street and complete the turn from there

Other (please describe):

I don't ever make right turns off of $L$ Street 
If you selected more than one option above, please explain how you determine which option to use?

\section{Loading or Drop-Off Zones}

At some mid-block locations, such as at hotel loading zones, bike lane markings direct bicyclists to move slightly away from the curb, and then back (see picture below).

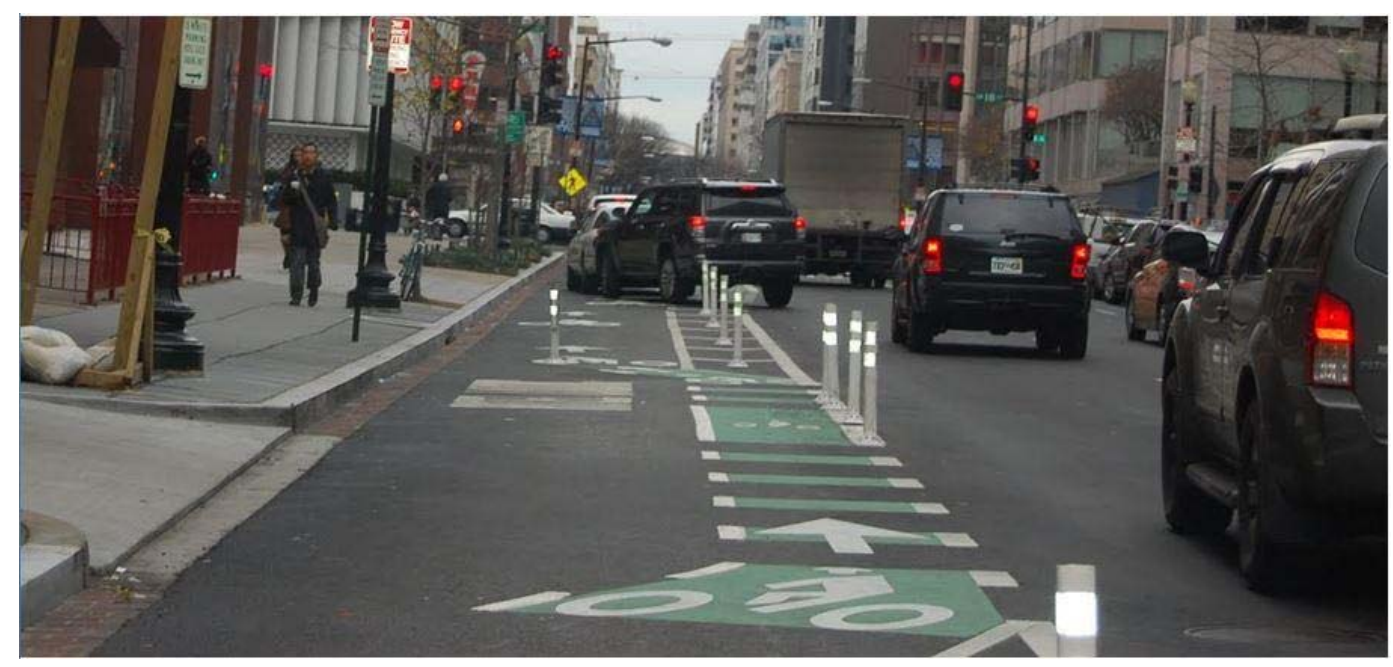

Please indicate if you agree or disagree with the following statements about the loading zone:

\begin{tabular}{|c|c|c|c|c|c|}
\hline & $\begin{array}{l}\text { Strongly } \\
\text { Disagree }\end{array}$ & $\begin{array}{l}\text { Somewhat } \\
\text { Disagree }\end{array}$ & $\begin{array}{l}\text { Somewhat } \\
\text { Agree }\end{array}$ & $\begin{array}{l}\text { Strongly } \\
\text { Agree }\end{array}$ & No Opinion \\
\hline $\begin{array}{r}\text { I often encounter motor vehicles stopped in these } \\
\text { loading zones. }\end{array}$ & 0 & ○ & ○ & 0 & 0 \\
\hline $\begin{array}{l}\text { Stopped cars at these loading zones usually allow } \\
\text { enough space for bicyclists to pass on the right. }\end{array}$ & 0 & ○ & 0 & 0 & 0 \\
\hline $\begin{array}{r}\text { Most motorists pay attention to bicyclists when entering } \\
\text { and exiting the loading zone area. }\end{array}$ & 0 & 0 & 0 & 0 & 0 \\
\hline $\begin{array}{r}\text { I feel comfortable bicycling around cars in the loading } \\
\text { zones. }\end{array}$ & O & ○ & 0 & ○ & 0 \\
\hline
\end{tabular}

\section{Overall Impressions of the Cycle Track}

What is the best thing about the cycle track?

What should be improved about the cycle track? 


\section{About Bicycling and Comfort}

Regardless of whether you currently bicycle in all of the following situations, please consider how comfortable you would be riding a bicycle in each place:

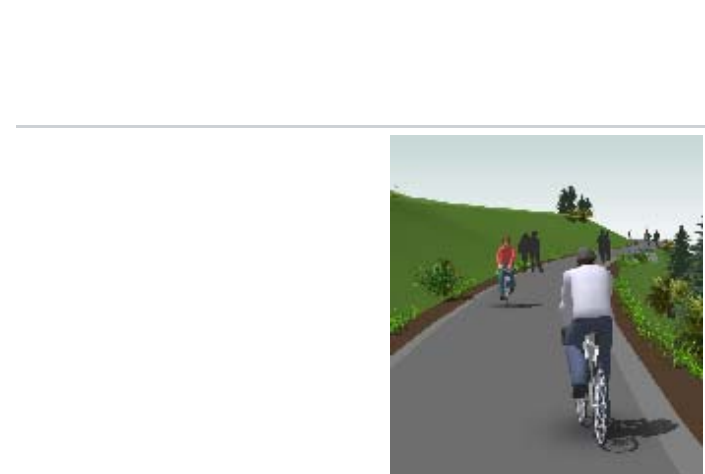

(A) On a path or trail separate from the street

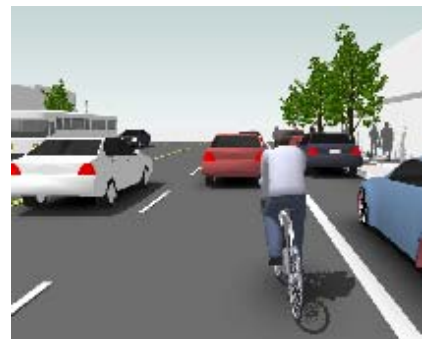

(B) On a commercial street with two lanes of traffic in each direction, with traffic speeds of 35 miles per hour, on-street car parking, and no bike lane

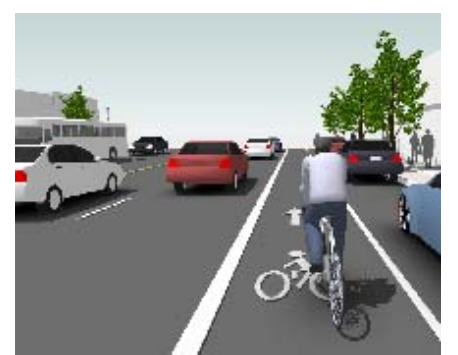

(C) On a similar street to (B), but with a striped bike lane
added

(C) On a similar street to $(\mathrm{B})$, but with a striped bike lane
added
Very Uncomfortable

(1)

(2)

(3)

(4)

(5)

Very omfortable

(6)

Very
Very

(1) Uncomfortable
(2)

(3)

(4)

(5) Comfortable

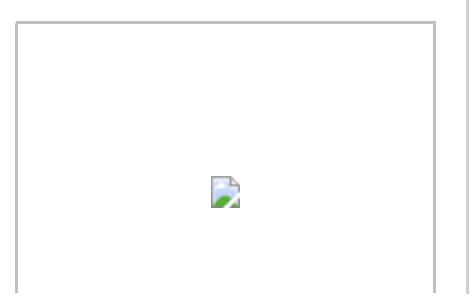


(D) On a similar street to $(B)$, but with a physically separated bike lane

How comfortable would you feel bicycling on a commercial street with two lanes of traffic in each direction, with traffic speeds of 35 miles per hour (Situation D above), but with the following types of separation from traffic:

Very

Uncomfortable

(1)
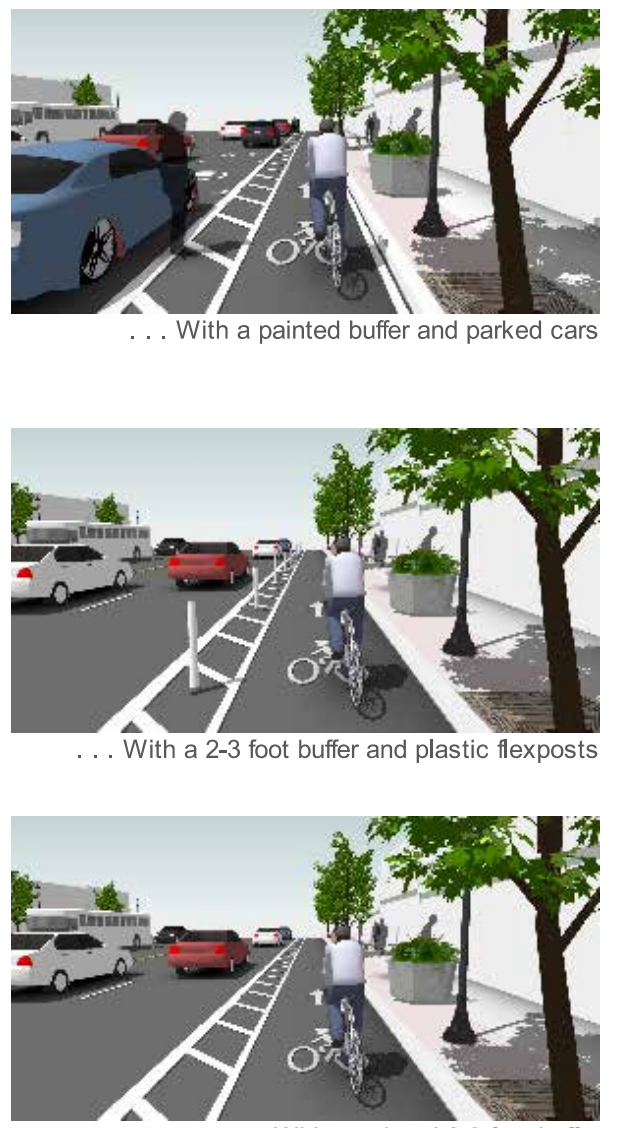

... With a painted 2-3 foot buffer
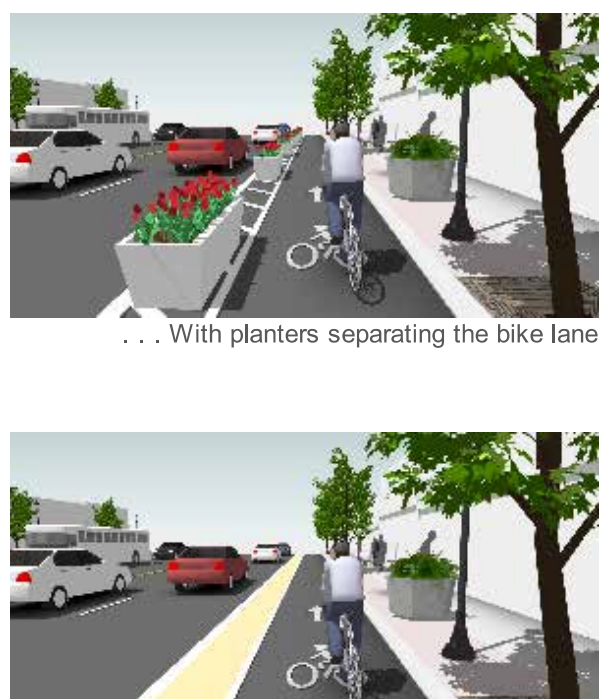

Very Uncomfortable

(1)

(2)

(3)

(4)

(5) Very

(6)
Very

Comfortable

(6) 

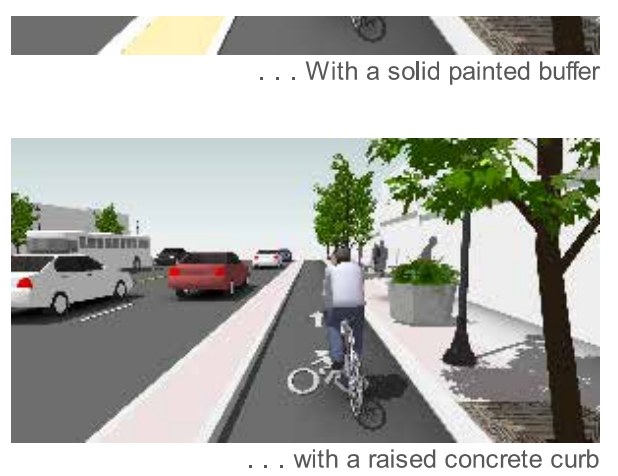

\section{About People you Encounter on the Street}

Indicate if you agree or disagree with the following statements:

\begin{tabular}{|c|c|c|c|c|c|}
\hline & $\begin{array}{l}\text { Strongly } \\
\text { Disagree }\end{array}$ & $\begin{array}{c}\text { Somewhat } \\
\text { Disagree }\end{array}$ & Somewhat Agree & Strongly Agree & No Opinion \\
\hline Most drivers follow the rules of the road. & O & O & O & O & ○ \\
\hline Most drivers are predictable. & ○ & O & O & ○ & O \\
\hline Most bicyclists follow the rules of the road. & O & ○ & ○ & ○ & ○ \\
\hline Most bicyclists are predictable. & ○ & ○ & O & O & ○ \\
\hline Most pedestrians follow the rules of the road. & ○ & ○ & ○ & ○ & ○ \\
\hline Most pedestrians are predictable. & O & O & O & O & O \\
\hline
\end{tabular}

\section{About You}

We have a few questions about you so that we may understand the characteristics of our survey respondents.

Do you have a current:

\begin{tabular}{|c|c|c|}
\hline & Yes & No \\
\hline Driver's License & 0 & O \\
\hline Transit Pass & ○ & ○ \\
\hline Bike-share membership & ○ & ○ \\
\hline Car-share membership & 0 & O \\
\hline
\end{tabular}

How many working motor vehicles does your household own or lease? (Do not include motorhomes).

$$
\begin{array}{r}
\# \text { Vehicles } \\
\text { (Enter } 0 \text { if None) }
\end{array}
$$

How many working adult bicycles does your household own?

$$
\begin{array}{r}
\text { \# Bicycles } \\
\text { (Enter } 0 \text { if None) }
\end{array}
$$

What is your home zip code? 
INCLUDING YOURSELF, how many people live in your household?

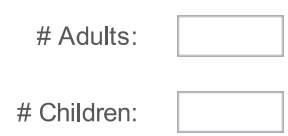

What is your gender?
Male
Female

○

○

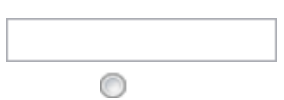

What is your age?

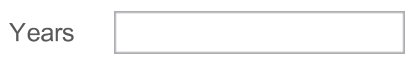

Do you consider yourself: (select all that apply)

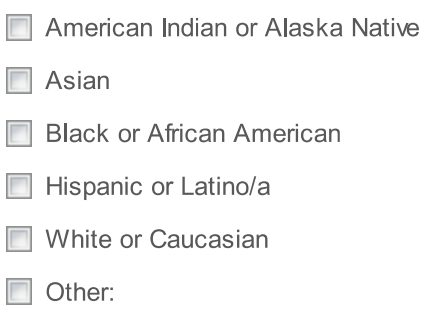

What is your employment status? (Select all that apply)

$\square$ I work outside the home -> What is the zip code of your place of work?

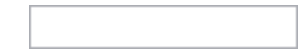

I go to school outside the home -> What is the zip code of your school?

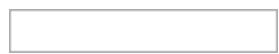

I work from home

Not at this time

What is your annual household income?

Less than $\$ 25,000$

$\$ 25,000$ to less than $\$ 50,000$

$\$ 50,000$ to less than $\$ 75,000$

- $\$ 75,000$ to less than $\$ 100,000$

$\$ 100,000$ to less than $\$ 200,000$

(1) $\$ 200,000$ or more

I prefer not to provide this information 
What is the highest level of school you havecompleted?

Some high school or less

High school diploma or GED

Some College

Trade/Vocational School

Associate Degree

Four-year college degree or more

Other (please specify):

Is there anything else that you would like to tell us?

That's all the questions we have. Would you like to be entered into a drawing for one of three $\$ 100$ gift cards to Amazon.com?

(2) Yes, I would like to be entered in the drawing ('IIl enter my name on the next page).

No thanks. I will complete my survey by clicking the ">>" button below

Enter your name and some way for us to get a hold of you below. We will only use this information to contact you if your entry is selected to receive a gift card. This information will not be associated with any of your survey responses.

Name

Phone or email

Thanks for taking the survey - please make sure to click the ">>" button below to submit your entry and close the survey! 